\title{
Assessment of Existing Transportation Packages for Use with HALEU
}

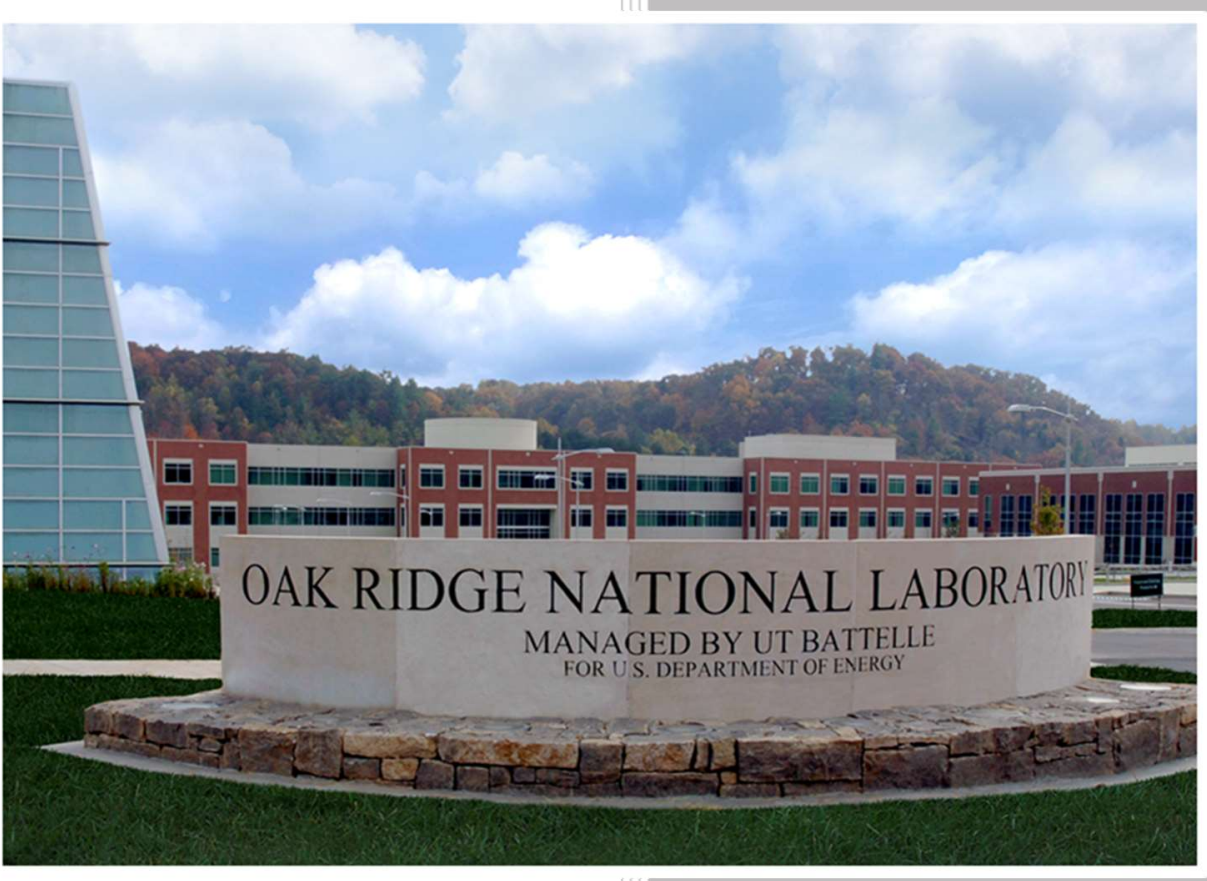

Approved for public release.

Robert Hall

B. J. Marshall

William A. Wieselquist

Distribution is unlimited.

September 2020 


\section{DOCUMENT AVAILABILITY}

Reports produced after January 1, 1996, are generally available free via US Department of Energy (DOE) SciTech Connect.

Website www.osti.gov

Reports produced before January 1, 1996, may be purchased by members of the public from the following source:

National Technical Information Service

5285 Port Royal Road

Springfield, VA 22161

Telephone 703-605-6000 (1-800-553-6847)

TDD 703-487-4639

Fax 703-605-6900

E-mail info@ntis.gov

Website http://classic.ntis.gov/

Reports are available to DOE employees, DOE contractors, Energy Technology Data Exchange representatives, and International Nuclear Information System representatives from the following source:

Office of Scientific and Technical Information

PO Box 62

Oak Ridge, TN 37831

Telephone 865-576-8401

Fax 865-576-5728

E-mail reports@osti.gov

Website http://www.osti.gov/contact.html

This report was prepared as an account of work sponsored by an agency of the United States Government. Neither the United States Government nor any agency thereof, nor any of their employees, makes any warranty, express or implied, or assumes any legal liability or responsibility for the accuracy, completeness, or usefulness of any information, apparatus, product, or process disclosed, or represents that its use would not infringe privately owned rights. Reference herein to any specific commercial product, process, or service by trade name, trademark, manufacturer, or otherwise, does not necessarily constitute or imply its endorsement, recommendation, or favoring by the United States Government or any agency thereof. The views and opinions of authors expressed herein do not necessarily state or reflect those of the United States Government or any agency thereof. 
Reactor and Nuclear Systems Division

\title{
ASSESSMENT OF EXISTING TRANSPORTATION PACKAGES FOR USE WITH HALEU
}

\author{
Robert Hall, B. J. Marshall, William A. Wieselquist
}

Date Published: October 2020

Prepared by

OAK RIDGE NATIONAL LABORATORY

Oak Ridge, TN 37831-6283

managed by

UT-BATTELLE, LLC

for the

US DEPARTMENT OF ENERGY

under contract DE-AC05-00OR22725 


\section{CONTENTS}

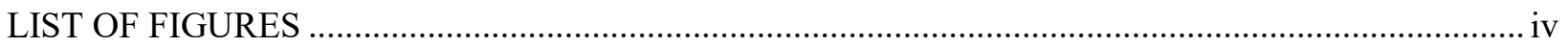

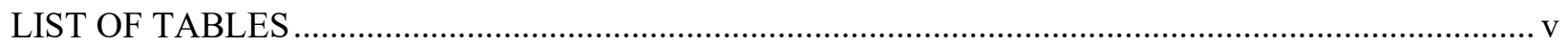

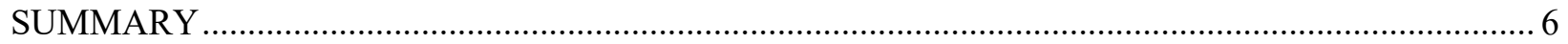

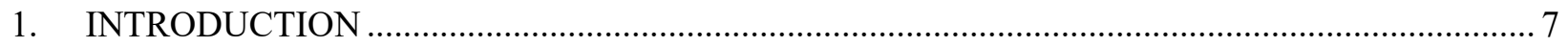

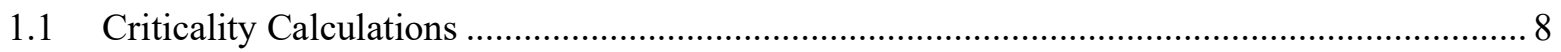

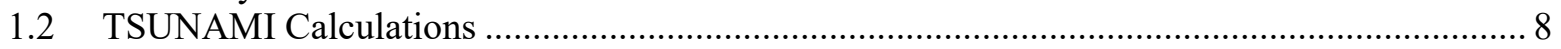

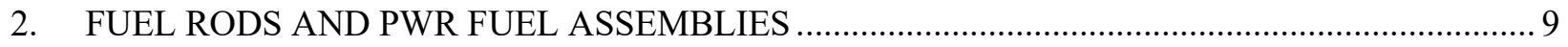

2.1 Westinghouse Traveller Package Description ..................................................................... 9

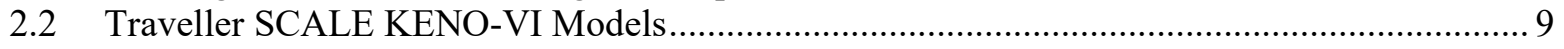

2.3 Traveller Fuel Assembly Model Criticality Calculations ..................................................... 15

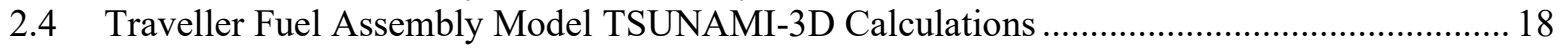

2.5 Traveller Fuel Assembly Model TSUNAMI-IP Results.................................................. 21

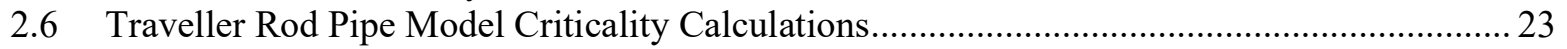

2.7 Traveller Rod Pipe Model TSUNAMI-3D Calculations ....................................................... 24

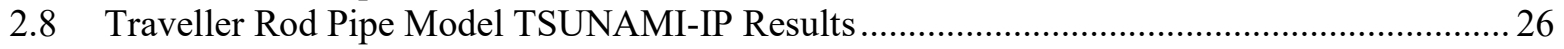

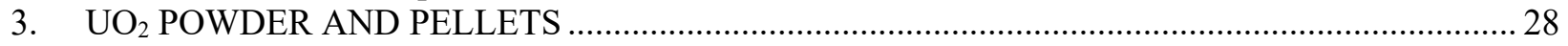

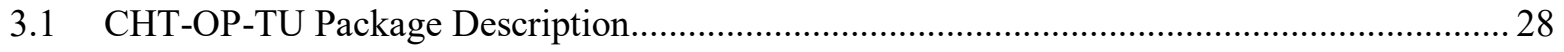

3.2 CHT-OP-TU Powder Model Criticality Calculations ............................................................ 31

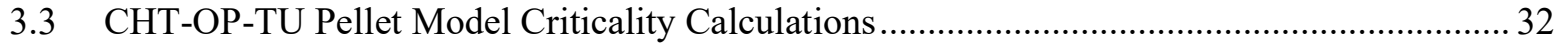

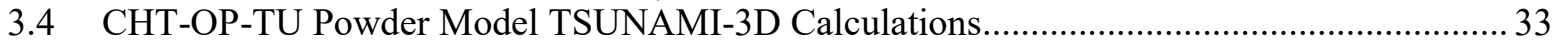

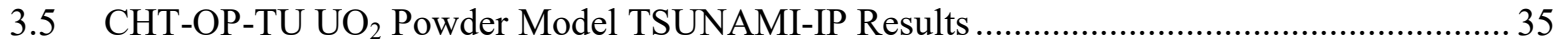

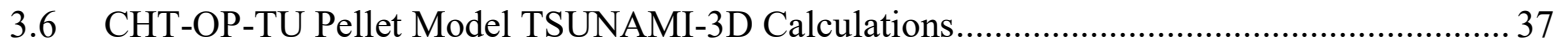

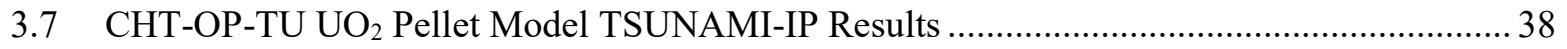

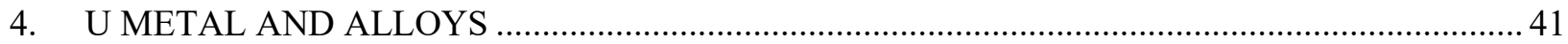

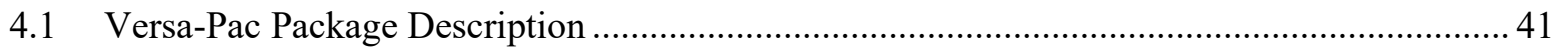

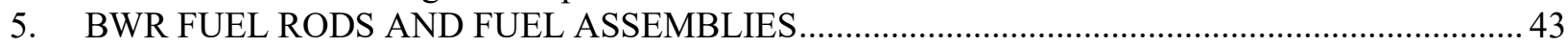

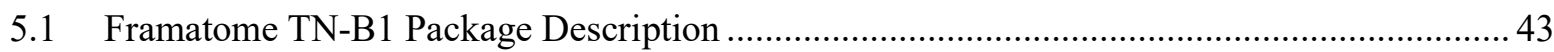

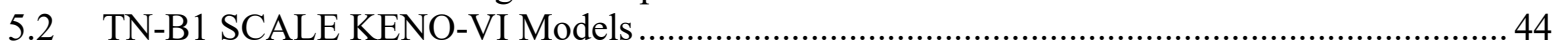

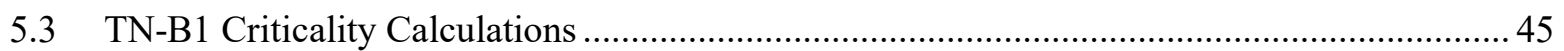

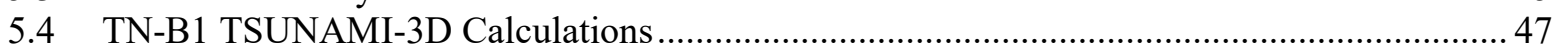

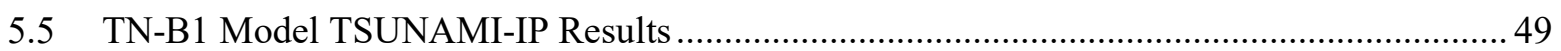

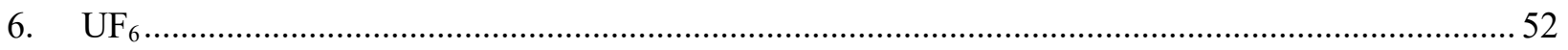

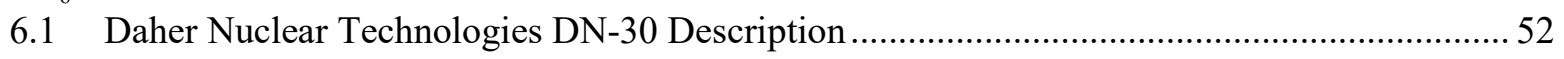

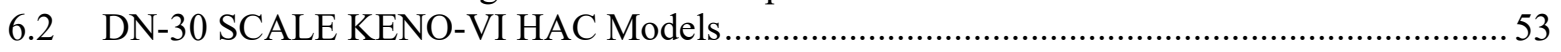

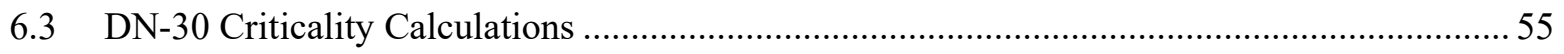

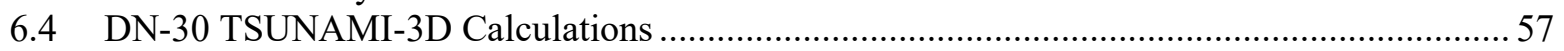

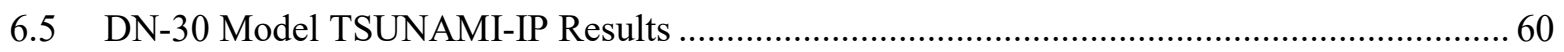

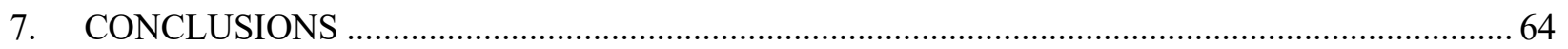

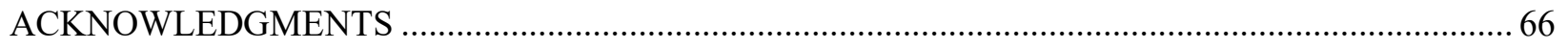

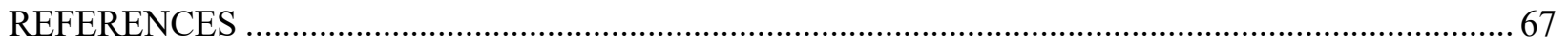

APPENDIX A. BENCHMARK EXPERIMENT SIMILARITY TABLE ……............................... A-1

APPENDIX B. TN-B1 WATER MODELING EFFECT ON BENCHMARK EXPERIMENT $c_{k} \ldots \ldots \ldots . . . . \mathrm{B}-1$ APPENDIX C. TN-B1 ARRAY SIZE MODELING EFFECT ON BENCHMARK EXPERIMENT

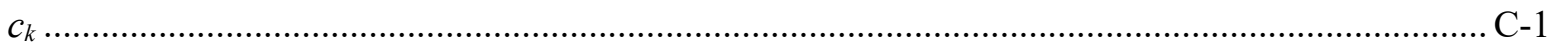

APPENDIX D. COMPUTER INPUT AND OUTPUT FILES ……............................................... 


\section{LIST OF FIGURES}

Figure 1. Westinghouse Traveller Standard package ...................................................................... 9

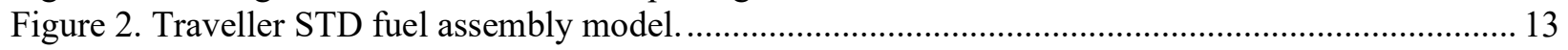

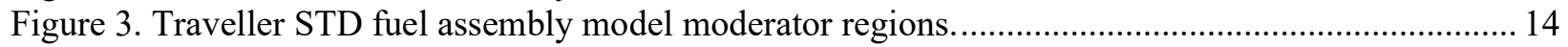

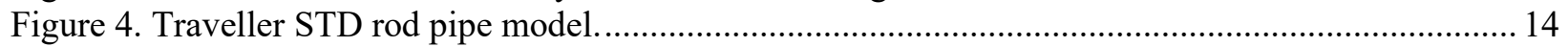

Figure 5. Sensitivity of the infinite array of packages with $5 \mathrm{wt} . \%$ fuel assemblies. ............................... 18

Figure 6. Sensitivity of the single package model with an $8 \mathrm{wt} . \%$ fuel assembly.................................... 19

Figure 7. Sensitivity of the infinite array of packages with 8 wt.\% 52 IFBA rod fuel assemblies............. 19

Figure 8. Traveller $c_{k}$ for the infinite array of packages with $5 \mathrm{wt} . \%$ fuel assemblies............................. 21

Figure 9. Traveller $c_{k}$ for a single package with an $8 \mathrm{wt} \%$ fuel assembly. ...........................................2 22

Figure 10. Traveller $c_{k}$ for the infinite array of packages with 8 wt.\% 52 IFBA rod fuel assemblies.

Figure 11. Rod pipe model $k_{\text {eff }}$ for fuel pellet diameter and rod pitch combinations for $5 \mathrm{wt} . \%$ $\mathrm{UO}_{2}$. 23

Figure 12. Rod pipe model $k_{\text {eff }}$ for fuel pellet diameter and rod pitch combinations for $10 \mathrm{wt} . \%$

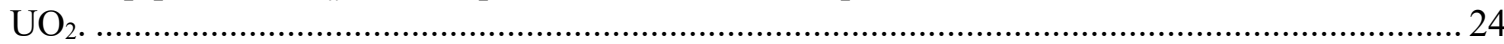

Figure 13. Sensitivity of the infinite array of packages with $5 \mathrm{wt} . \% \mathrm{UO}_{2}$ fuel rods...............................25

Figure 14. Sensitivity of the infinite array of packages with $10 \mathrm{wt} \% \mathrm{UO}_{2}$ fuel rods...............................25

Figure 15. Traveller $c_{k}$ for the infinite array of packages with $5 \mathrm{wt} . \% \mathrm{UO}_{2}$ fuel rods.............................26

Figure 16. Traveller $c_{k}$ for the infinite array of packages with $10 \mathrm{wt} . \% \mathrm{UO}_{2}$ fuel rods........................... 27

Figure 17. CHT-OP-TU model $\mathrm{UO}_{2}$ powder model $(4 \times 4 \times 3$ package array) .......................................2 29

Figure 18. CHT-OP-TU UO UO $_{2}$ powder model optimum moderation results........................................... 30

Figure 19. CHT-OP-TU UO $\mathrm{UO}_{2}$ pellet model optimum moderation results. ............................................... 31

Figure 20. CHT-OP-TU UO U $_{2}$ powder model sensitivity (48 packages, 5 wt.\%, 8 in. OV).................... 34

Figure 21. CHT-OP-TU UO 2 powder model sensitivity (18 packages, $8 \mathrm{wt} . \%, 7.5$ in. OV)................... 34

Figure 22. CHT-OP-TU $c_{k}$ for 5 wt.\% $\mathrm{UO}_{2}$ powder (48 packages, 8 in. diameter OV)........................ 35

Figure 23. CHT-OP-TU $c_{k}$ for 8 wt.\% $\mathrm{UO}_{2}$ powder (18 packages, 7.5 in. diameter $\mathrm{OV}$ )....................... 36

Figure 24. CHT-OP-TU UO 2 pellet model sensitivity (18 packages, 6.9 wt.\%, 7.5 in. OV)................... 37

Figure 25. CHT-OP-TU UO 2 pellet model sensitivity (48 packages, $16.5 \mathrm{wt} . \%, 6$ in. OV)...................... 38

Figure 26. CHT-OP-TU $c_{k}$ for 6.9 wt.\% $\mathrm{UO}_{2}$ pellets (18 packages, 7.5 in. diameter OV)....................... 39

Figure 27. CHT-OP-TU $c_{k}$ for 16.5 wt.\% $\mathrm{UO}_{2}$ pellets (48 packages, 6 in. diameter OV)....................... 39

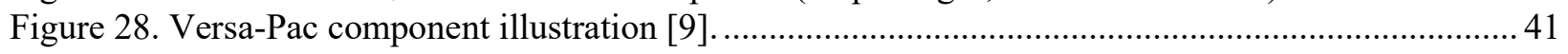

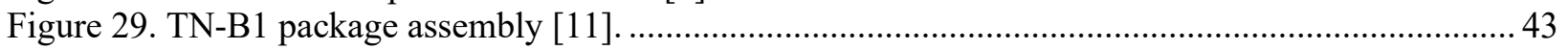

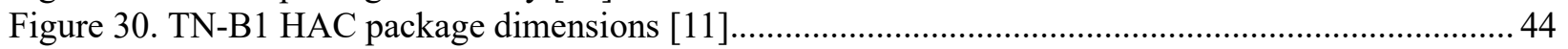

Figure 31 TN-B1 $6 \times 1 \times 6$ package array top and front cutaway views ............................................ 45

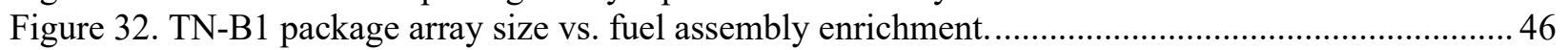

Figure 33 . TN-B1 $10 \times 1 \times 10$ package Gd rods required vs. fuel assembly enrichment........................ 46

Figure 34. TN-B1 model sensitivity (100 packages, 5 wt.\%, $13 \mathrm{Gd}$ rods/fuel assembly)........................ 48

Figure 35. TN-B1 model sensitivity (36 packages, $6.7 \mathrm{wt} \% \%, 13 \mathrm{Gd}$ rods/fuel assembly)...................... 48

Figure 37 . TN-B1 model sensitivity (100 packages, $8 \mathrm{wt} \%$, $24 \mathrm{Gd}$ rods/fuel assembly)........................ 48

Figure 37. TN-B1 $c_{k}$ for $5 \mathrm{wt} . \% \mathrm{UO}_{2}(100$ packages, $13 \mathrm{Gd}$ rods/fuel assembly)................................... 49

Figure 38. TN-B1 $c_{k}$ for $6.7 \mathrm{wt} . \% \mathrm{UO}_{2}$ (36 packages, $13 \mathrm{Gd}$ rods/fuel assembly)..................................50

Figure 39. TN-B1 $c_{k}$ for $8 \mathrm{wt} . \% \mathrm{UO}_{2}(100$ packages, $24 \mathrm{Gd}$ rods/fuel assembly).................................. 50

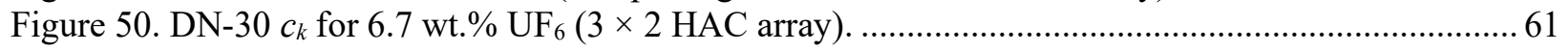

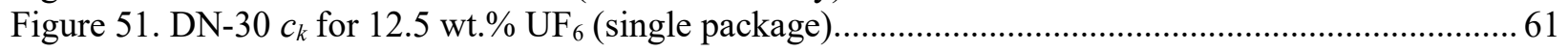




\section{LIST OF TABLES}

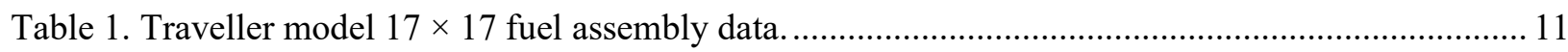

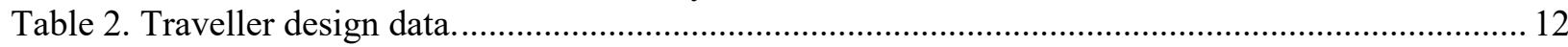

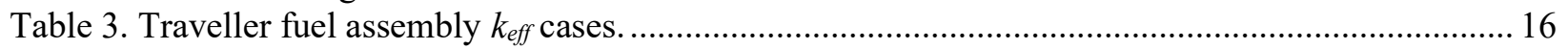

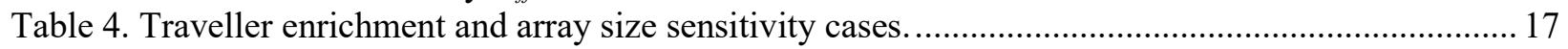

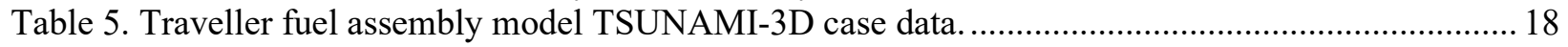

Table 6. Traveller fuel assembly model sensitivity by mixture ................................................................ 20

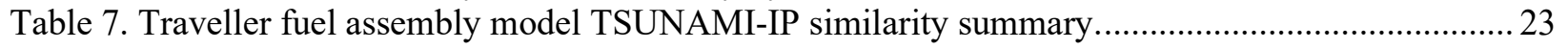

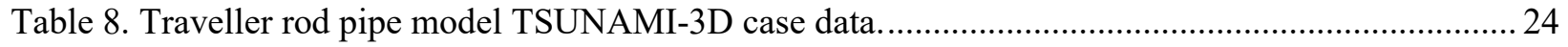

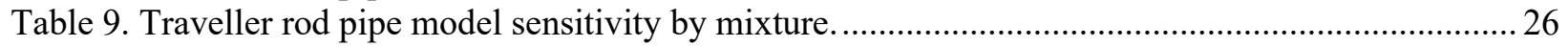

Table 10. Traveller rod pipe model TSUNAMI-IP similarity summary .................................................. 27

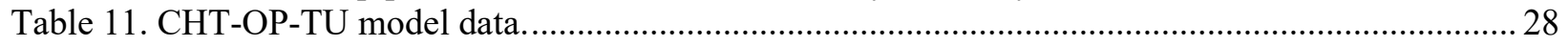

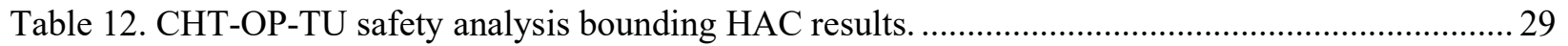

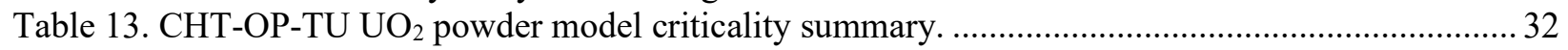

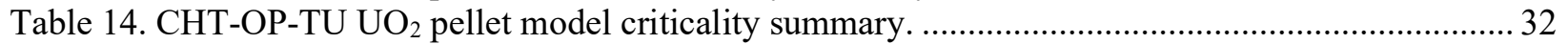

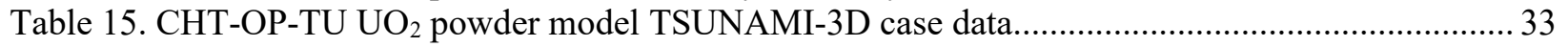

Table 16. CHT-OP-TU UO $\mathrm{UO}_{2}$ powder model sensitivity by mixture ...................................................... 35

Table 17. CHT-OP-TU UO $\mathrm{U}_{2}$ powder model TSUNAMI-IP similarity summary. ..................................... 36

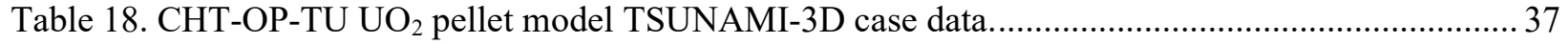

Table 19. CHT-OP-TU UO $\mathrm{UO}_{2}$ pellet model sensitivity by mixture.......................................................... 38

Table 20. CHT-OP-TU UO U $_{2}$ pellet model TSUNAMI-IP similarity summary. ...................................... 40

Table 21. Versa-Pac maximum ${ }^{235} \mathrm{U}$ per package (ground/vessel transportation) [15] .......................... 42

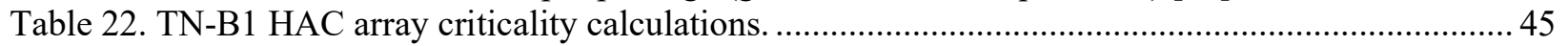

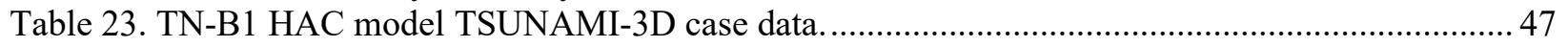

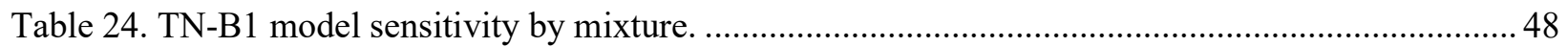

Table 25. TN-B1 model TSUNAMI-IP similarity summary......................................................... 51

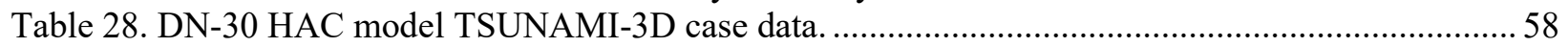

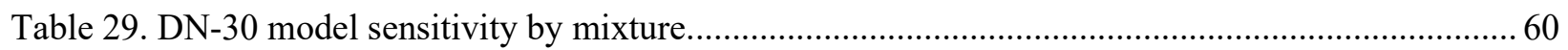




\section{SUMMARY}

Commercial light water reactor operators and fuel vendors in the United States are pursuing changes to fuel that include increased ${ }^{235} \mathrm{U}$ enrichment. Economic studies generally anticipate maximum near-term fuel assembly designs with up to $8 \mathrm{wt} . \%{ }^{235} \mathrm{U}$. Many next-generation nuclear reactor designs require highassay low-enriched uranium (HALEU) (19.75 wt.\% $\left.>{ }^{235} \mathrm{U}>5 \mathrm{wt} . \%\right)$ fuel. One necessary element for the commercial-scale use of HALEU is the ability to safely transport large quantities of enriched fuel material in multiple forms. However, there is uncertainty as to whether subcriticality requirements can be satisfied with existing package designs and whether existing critical benchmark experiment data are sufficient to support criticality safety code validation for HALEU transportation applications.

This study assesses the potential to use currently licensed transportation packages for the transportation of increased enrichment unirradiated $U$ fuel forms. The assessment uses selected package designs that represent the five categories of fuel form - boiling water reactor pins and assemblies, pressurized water reactor pins and assemblies, $\mathrm{UF}_{6}, \mathrm{U}-$ metal and tristructural isotropic (TRISO) particles, and $\mathrm{UO}_{2}$ pellets or powder - and focuses on demonstrating subcriticality and identifying benchmark critical experiments appropriate for use in criticality computer code validation.

Key quantities of interest that relate to subcriticality are limiting conditions (e.g., optimum moderation), package or package array $k_{\text {eff, }}$ package capacity, and package transportation array size. The SCALE TSUNAMI-3D and TSUNAMI Indices and Parameters codes are used for sensitivity and uncertainty calculations and for identification of candidate critical benchmark experiments for code validation. The primary metric for identifying candidate benchmarks is the similarity coefficient, $c_{k}$.

For each fuel form category, a representative package is evaluated. Results provided for each package evaluation include enrichment and packaging limits (e.g., maximum transportation array size as a function of enrichment) and benchmark critical experiment similarity coefficients. Results indicate that there are viable means for increasing enrichments into the HALEU range across the spectrum of fuel forms with differing increase amounts available for different packages. Sources of subcriticality margin to offset increased enrichment reactivity include reduced transportation array size, reduced fissile mass, burnable absorber credit, and safety analysis margin harvesting. For all packages except the DN-30, numerous critical benchmark experiment candidates for validation were identified. 


\section{INTRODUCTION}

Commercial light water reactor (LWR) operators and fuel vendors in the United States are pursuing changes to fuel that include increased ${ }^{235} \mathrm{U}$ enrichment. [1,2]. Economic studies generally anticipate near-term fuel assembly average enrichment up to $8 \mathrm{wt} . \%{ }^{235} \mathrm{U}$, which is within the range of high-assay low-enriched uranium (HALEU) $\left(19.75 \%>{ }^{235} \mathrm{U}>5 \%\right.$ ). Many next-generation nuclear reactor designs require HALEU fuel. One necessary element for the commercial-scale use of HALEU is the ability to safely transport large quantities of enriched fuel material in multiple forms. However, there is uncertainty as to whether subcriticality requirements can be satisfied with existing package designs and whether existing critical benchmark experiment data are sufficient to support criticality safety code validation for HALEU transportation applications.

To prepare for and support these potential changes, the potential use of currently licensed transportation packages for the transportation of EE and HALEU is being assessed. This assessment includes selected package designs that represent the five categories of fuel form-boiling water reactor (BWR) pins and assemblies, pressurized water reactor (PWR) pins and assemblies, $\mathrm{UF}_{6}, \mathrm{U}$-metal and tristructural isotropic (TRISO), and $\mathrm{UO}_{2}$ pellets or powder-and are limited to unirradiated $\mathrm{U}$ fuel forms. This assessment focuses on modeling the limiting packaging conditions with current enrichment limits and modifying those models to include potential HALEU enrichments to identify criticality and validation effects.

The primary focus of this study is to determine whether existing package designs can be used to transport increased HALEU LWR fuel materials, what transportation limitations might be required, and whether sufficient applicable benchmark critical experiments can be identified for computer code validation. The SCALE KENO-VI code is used for criticality calculations [3]. Key quantities of interest relating to subcriticality are limiting conditions (e.g., optimum moderation), package or package array $k_{\text {eff }}$, package capacity, and package transportation array size. The SCALE TSUNAMI-3D and TSUNAMI Indices and Parameters (TSUNAMI-IP) codes are used for sensitivity and uncertainty calculations and for identification of candidate critical benchmark experiments for code validation. [3,4.] The primary metric for identifying candidate benchmarks is the similarity coefficient, $c_{k}$.

For each category of fuel form, a representative package is evaluated.

1) Traveller (PWR fuel assemblies, PWR and BWR fuel pins) $[5,6]$.

2) CHT-OP-TU ( $\mathrm{UO}_{2}$ powder and pellets) $[7,8]$.

3) VersaPac (U-metal/TRISO) $[9,10]$.

4) TN-B1 (BWR fuel assemblies) $[11,12]$.

5) $\mathrm{DN}-30\left(\mathrm{UF}_{6}\right)[13,14]$

Results provided for each package evaluation include the enrichment and packaging limits (e.g., maximum transportation array size as a function of enrichment) and identification of benchmark critical experiments that are appropriate for use in computer code validation (determination of $k_{\text {eff }}$ bias and bias uncertainty for the application). 


\subsection{Criticality Calculations}

For each package, KENO-VI criticality calculations are performed by using the following process.

1) Construct package and package array models by using Certificate of Compliance (CoC) and safety analysis report (SAR) information.

a. The hypothetical accident condition (HAC) array model is typically limiting.

2) Confirm limiting conditions and establish maximum target $k_{\text {eff. }}$.

a. Target $k_{\text {eff }}$ is chosen to preserve margin to the upper subcritical limit from the safety analysis with current package enrichment limit (e.g., 5 wt. $\%{ }^{235} \mathrm{U}$ ).

3) Calculate $k_{\text {eff }}$ varying enrichment and package array size.

a. Burnable absorbers, fuel rod pitch, optimum moderation, and package capacity might also be varied, as applicable.

b. Determine model variations with $k_{\text {eff }}$ close to the target.

4) Select representative enrichment, array size, and other acceptable increased enrichment packaging variations for TSUNAMI analysis.

KENO $k_{\text {eff }}$ convergence is typically set for scoping-level results (i.e., $k_{\text {eff }}$ standard deviation of 0.001 ) for these calculations. Optimum moderation searches are automated by using the SCALE SAMPLER stochastic sampling sequence. Criticality calculations use KENO-VI in SCALE version 6.3 pre-release beta 11 with the 252 group ENDF/B-VII.1 cross section library.

\subsection{TSUNAMI Calculations}

The SCALE TSUNAMI suite of tools performs sensitivity and uncertainty (S/U) calculations [4]. TSUNAMI-3D uses adjoint-based perturbation theory to calculate the energy and reaction-dependent sensitivity of a system $k_{\text {eff }}$ to changes in cross section data. A sensitivity data file (SDF) that contains the calculated sensitivity coefficients is produced for the application of interest and as a set of benchmark critical experiments. TSUNAMI-IP combines sensitivity data with nuclear data uncertainty information to produce a correlation coefficient, $c_{k}$, for each application experiment pair. The $c_{k}$ value indicates the degree to which the $k_{\text {eff }}$ of an application and the $k_{\text {eff }}$ of an experiment are correlated in sensitivity to cross section perturbation or to cross section data uncertainty.

A $c_{k}$ value of 1.0 indicates perfectly correlated models, and a value of zero indicates that the two models share no neutronic similarity. For the purpose of computer code validation to establish criticality safety $k_{\text {eff }}$ bias and bias uncertainty, experiments and applications are considered similar for $c_{k}$ values $\geq 0.9$ and marginally similar for $c_{k}$ values $\geq 0.8[15,16]$.

A suite of 1,584 critical experiments with LEU or intermediate-enriched uranium (IEU) is used with TSUNAMI-IP to identify potentially applicable experiments for $k_{\text {eff }}$ validation. Within the International Criticality Safety Benchmark Evaluation Project (ICSBEP) Handbook [17], IEU is defined as uranium enriched to $10-60$ wt.\% ${ }^{235} \mathrm{U}$. SDFs for these systems are distributed with the ICSBEP Handbook. Onehundred and eleven SDFs were drawn from the VALID library maintained at ORNL [18]. The ICSBEP SDFs are considered sufficiently accurate for experiment selection [19]. The ICSBEP experiment naming convention indicates the enrichment range, material form, and energy spectrum (i.e., LCT represents LEU, compound form, and thermal energy spectrum).

SCALE version 6.3 pre-release beta 11 was used for all calculations. 


\section{FUEL RODS AND PWR FUEL ASSEMBLIES}

\subsection{Westinghouse Traveller Package Description}

The Westinghouse Traveller package is the representative design chosen to assess the transportation of PWR fuel pins and fuel assemblies [5, 6]. There are several versions of the Traveller: Standard (STD), $\mathrm{XL}$, and VVER. For this analysis, the Standard version was used because it can carry the most common length of PWR fuel (12 ft of active fuel).

The Traveller is designed to transport one commercial-grade unirradiated U PWR fuel assembly with enrichment up to $5.0 \mathrm{wt} . \%{ }^{235} \mathrm{U}$ and unirradiated BWR and PWR fuel rods in a rod pipe. The primary packaging components are an Outerpack and a hinged Al Clamshell that can accommodate one fuel assembly or one rod pipe. The Clamshell includes Boral neutron absorber plates, and the Outerpack contains polyethylene moderator blocks. The combination of moderator blocks and absorber plates provides criticality control across a range of dry and flooded conditions. PWR assembly packing materials are limited to $\leq 2.0 \mathrm{~kg}$ per package. A representation of an empty Traveller package is shown in Figure 1.
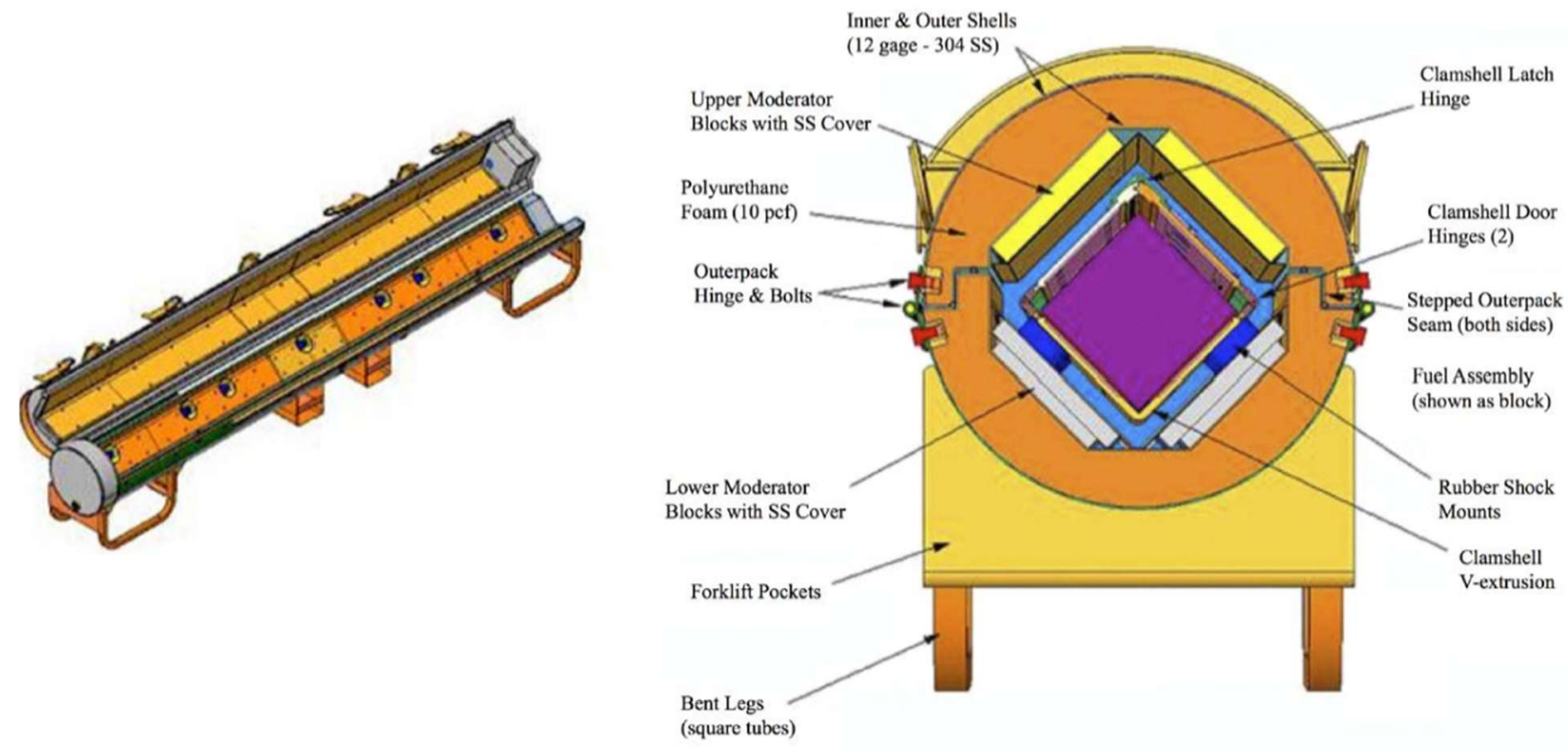

Figure 1. Westinghouse Traveller Standard package. [5]

The rod pipe is a 6 in. Schedule 40304 stainless-steel (SS) pipe with SS closures at each end. Within the rod pipe, $\mathrm{UO}_{2}$ or $\mathrm{U}_{3} \mathrm{Si}_{2}$ fuel rods are wrapped in polyethylene sleeves with a $\mathrm{H}$ density of $\leq 0.1325 \mathrm{~g} / \mathrm{cm}^{3}$. Loose fuel rod packing material is unlimited in the rod pipe.

\subsection{Traveller SCALE KENO-VI Models}

Two models were used: one with a $17 \times 17$ Westinghouse fuel assembly centered in the Clamshell and one with $\mathrm{UO}_{2}$ rods in a rod pipe centered in the Clamshell. Only one fuel design was used because the objective of this calculation was to assess the effect of increased enrichment. This objective can be met with a representative fuel assembly. Determining a bounding assembly design for all conditions is complex and was outside the scope of this analysis. 
Many Traveller design features unrelated to criticality control are neglected in the KENO models. The elements of the package considered important to criticality that are included in the models are:

- Clamshell shell,

- Boral plates,

- polyethylene blocks,

- Outerpack SS covers,

- rod pipe,

- polyethylene packing in the rod pipe,

- preferential water flooding, and

- HAC fuel assembly damage (rod pitch increase).

The current Traveller SAR indicates that the most reactive flooding configuration is full-density water in the Clamshell, which includes within the fuel pin lattice, in the fuel-clad gap space, and in place of guide tube and instrument tube material. Void in all other regions maximizes reactivity, which indirectly supports modeling foam in the Outerpack as void.

The Traveller SAR evaluates multiple fuel assembly designs and numerous tolerance perturbations to the nominal package and fuel design (e.g., clad thickness, polyethylene block density, fuel rod pitch, axial positioning in the Clamshell, annular pellet region length) to obtain bounding penalties that do not necessarily apply to any single fuel assembly design. Because attempting to replicate the SAR approach is complex, nominal design values were used in this analysis with two exceptions: (1) a $50.8 \mathrm{~cm}$ region of the fuel assembly lattice was modeled as expanded into the available space to simulate fuel assembly damage observed during HAC drop testing and (2) non-fuel lattice locations (guide and instrument tubes) were modeled as water. The SAR analysis shows that HAC conditions are typically the most reactive. Therefore, this assessment used an HAC model with simulated fuel damage and optimum preferential region flooding. A $17 \times 17$ fuel assembly design is used to represent PWR designs.

For the rod pipe model, fuel pin gap and clad are modeled as moderator (polyethylene) and an optimum pitch, and pellet radius search was performed to maximize $k_{\text {eff }}$, which is consistent with the SAR.

For TSUNAMI-3D calculations, a single package with reflective boundary conditions or $30 \mathrm{~cm}$ water reflection was used instead of an array of packages to facilitate reasonable execution times and computer resource requirements. This accommodation is justified for two reasons: (1) most Traveller HAC arrays are large (24-150 packages) and (2) with preferential region flooding and Boral absorbers in the Clamshell, neutronic interaction between packages is low. Appendix $\mathrm{C}$ describes a limited study of the effect of using package arrays that are different from the bounding criticality array in TSUNAMI-3D calculations. Generally, more accurate similarity results are expected when the TSUNAMI and criticality models are the same.

Table 1 provides fuel assembly and material descriptions. Table 2 shows Clamshell and Outerpack dimensions and material descriptions. In the fuel assembly model, guide tubes and instrument tubes were modeled as moderators, and $2 \mathrm{~kg}$ of polyethylene packaging were included as a liner in the Clamshell. Similarly, in the rod pipe model, fuel pins were modeled as a triangular pitch $\mathrm{UO}_{2}$ lattice with no cladding. The non-fuel region in the pipe was modeled as polyethylene packaging. 
Table 1. Traveller model $17 \times 17$ fuel assembly data.

\begin{tabular}{ll}
\hline \multicolumn{1}{c}{ Parameter } & \multicolumn{1}{c}{ Value } \\
\hline Assembly lattice & $17 \times 17$ \\
\hline Assembly pitch & $21.5 \mathrm{~cm}$ \\
\hline Fuel rods & 264 \\
\hline Guide tubes & 24 \\
\hline Instrument tubes & 1 \\
\hline Fuel rod pitch & $1.26 \mathrm{~cm}$ (nominal) \\
\hline Fuel rod pitch & $1.355 \mathrm{~cm}($ damaged) \\
\hline $\mathrm{UO}_{2}$ pellet diameter & $0.7831 \mathrm{~cm}$ \\
\hline $\mathrm{UO}_{2}$ effective density & $10.96 \mathrm{~g} / \mathrm{cm}^{3}$ \\
\hline $\mathrm{Clad}_{\text {inner diameter }}$ & $0.7937 \mathrm{~cm}$ \\
\hline Clad outer diameter & $0.9004 \mathrm{~cm}$ \\
\hline Fuel stack height & $365 \mathrm{~cm}$ \\
\hline Temperature & $293 \mathrm{~K}$ \\
\hline Full water density & $0.9986 \mathrm{~g} / \mathrm{cm}^{3}$ \\
\hline
\end{tabular}


Table 2. Traveller design data.

\begin{tabular}{ll}
\hline \multicolumn{1}{c}{ Parameter } & \multicolumn{1}{c}{ Calue } \\
\hline Outside length & $439.4 \mathrm{~cm}$ \\
\hline Boral length & $426.7 \mathrm{~cm}$ \\
\hline Boral width & $15.2 \mathrm{~cm}$ \\
\hline Clamshell material & $\mathrm{Al}$ \\
\hline Clamshell wall thickness & $0.714 \mathrm{~cm}$ \\
\hline Clamshell inner height/width & $23.17 \mathrm{~cm}$ \\
\hline \multicolumn{1}{c}{ Outerpack } \\
\hline Outside length & $500.4 \mathrm{~cm}$ \\
\hline Outside diameter & $63.5 \mathrm{~cm}$ \\
\hline Outer shell material & $304 \mathrm{SS}$ \\
\hline Outer shell thickness & $0.266 \mathrm{~cm}$ \\
\hline Inner shell thickness & $0.266 \mathrm{~cm}$ \\
\hline Inner shell inside width ${ }^{*}$ & $43.2 \mathrm{~cm}$ \\
\hline Cavity length & $455.8 \mathrm{~cm}$ \\
\hline Polyethylene block length & $457.2 \mathrm{~cm}$ \\
\hline Upper poly block width & $24.1 \mathrm{~cm}$ \\
\hline Upper poly block thickness & $3.18 \mathrm{~cm}$ \\
\hline Large lower poly block width & $23.7 \mathrm{~cm}$ \\
\hline Large lower poly block thickness & $2.54 \mathrm{~cm}$ \\
\hline Small lower poly block width & $19.94 \mathrm{~cm}$ \\
\hline Small lower poly block thickness & $1.91 \mathrm{~cm}$ \\
\hline \multicolumn{1}{c}{ Rod pipe } \\
\hline Rod pipe outside diameter & $16.84 \mathrm{~cm}$ \\
\hline Rod pipe inside diameter & $15.41 \mathrm{~cm}$ \\
\hline Measured along horizontal centerline & \\
\hline
\end{tabular}


Figure 2 shows cutaway views of the Traveller standard model with a $17 \times 17$ fuel assembly centered in the Clamshell and with no fuel. Figure 3 shows the same model with individual moderator regions indicated by color. Separate moderator regions inside the Clamshell (red), inside the Outerpack (yellow), in the foam region (purple), and outside the Outerpack (blue) are used to determine the flooding configuration that maximizes $k_{\text {eff. }}$ The foam region is modeled as an annulus that is a simple approximation sufficient to confirm whether void, foam, or water results in the highest reactivity.
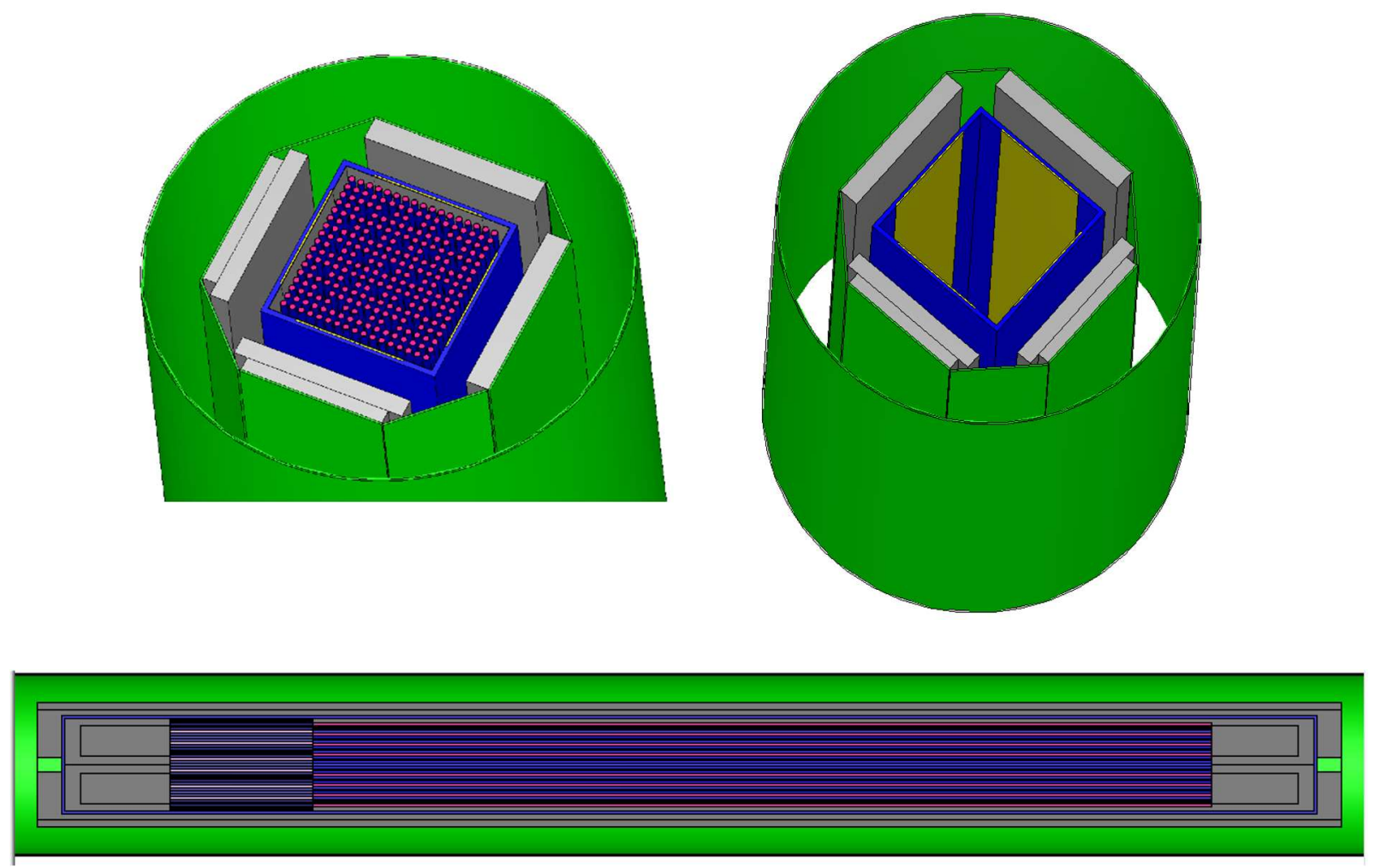

Figure 2. Traveller STD fuel assembly model. 

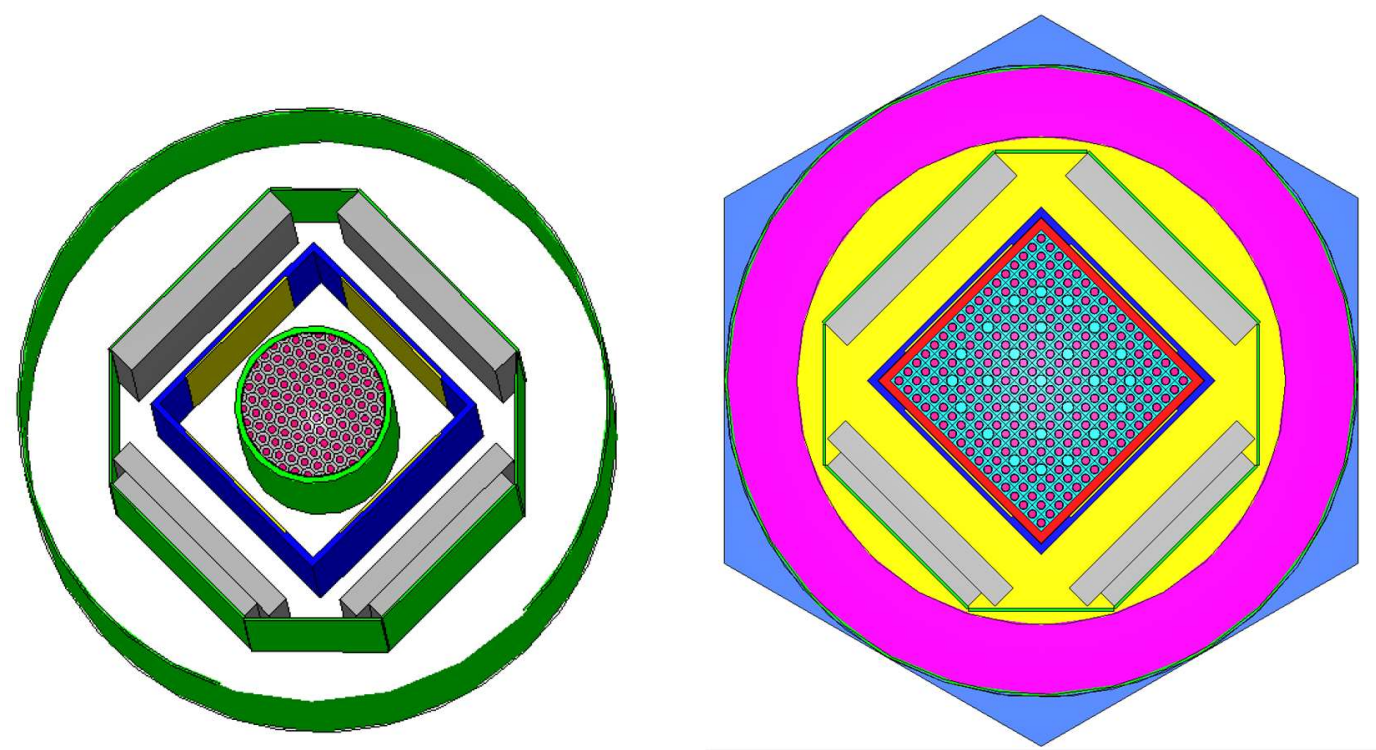

Figure 3. Traveller STD fuel assembly model moderator regions.

Figure 4 shows the rod pipe model. The rod pitch and rod diameter are variable and were used to identify the combination that maximizes $k_{\text {eff. }}$. Partial fuel rods bisected by the rod pipe are retained in the model for convenience.

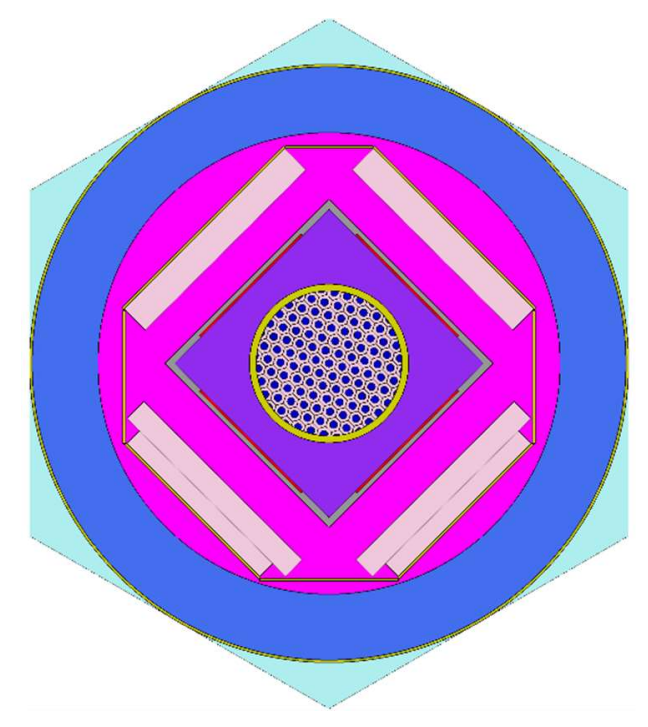

Figure 4. Traveller STD rod pipe model. 


\subsection{Traveller Fuel Assembly Model Criticality Calculations}

KENO-VI cases were run with various combinations of flooding to establish maximum $k_{\text {eff }}$ conditions. Preferential full-density water flooding inside the Clamshell, inside the Outerpack, in the foam region, and outside the Outerpack were considered separately and in combination. Outside the Outerpack, optimum-density water was also considered.

The Traveller SAR indicates that the maximum analyzed $k_{\text {eff }}$ with $5 \mathrm{wt} . \%$ fuel assemblies is close to the upper safety limit ( 0.94). The analysis bounds all versions of the Traveller package, all allowable fuel assembly types, maximum HAC array size, and all penalties. The Traveller STD model with $17 \times 17$ fuel used in this analysis is representative but is not bounding. Therefore, for this evaluation, the maximum $k_{\text {eff }}$ of the $5 \mathrm{wt} . \%$ base case was used as a target $k_{\text {eff }}$ for cases with higher enrichment fuel. The base case comprises an infinite array of packages with each package containing a $5 \mathrm{wt} . \% 17 \times 17$ fuel assembly. Increased enrichment will increase $k_{\text {eff }}$ well above the target with no other changes to the package. Two options were considered for reducing maximum $k_{\text {eff }}$ with up to $8 \mathrm{wt} . \%$ fuel: (1) reduced transportation array size and (2) integral fuel burnable absorber (IFBA) rods.

Table 3 shows the results of preferential flooding calculations for the base case KENO-VI infinite array model, which is a hexagonal array with the sides of the packages touching and reflective boundary conditions. Neutron spectrum hardness is indicated by the energy of the average lethargy of fission (EALF). Two different fuel enrichments were used: 5 and $8 \mathrm{wt} . \%{ }^{235} \mathrm{U}$. The highest $k_{\text {eff }}$ occured with the Clamshell, fuel lattice, and pellet-clad gap region flooded for both enrichments. The $8 \mathrm{wt} \%$ maximum $k_{\text {eff }}$ exceeded the target by about 0.06 . 
Table 3. Traveller fuel assembly $k$ eff cases.

\begin{tabular}{|c|c|c|c|c|c|c|c|}
\hline \multirow[b]{2}{*}{$\begin{array}{l}\text { Enrichment } \\
\left.\text { (wt.\% }{ }^{235} \mathbf{U}\right)\end{array}$} & \multicolumn{4}{|c|}{ Water flooding } & \multirow[b]{2}{*}{ Array } & \multirow[b]{2}{*}{$\boldsymbol{k}_{\text {eff }}$} & \multirow[b]{2}{*}{ EALF } \\
\hline & Clamshell $^{1}$ & Outerpack & Foam & External & & & \\
\hline 5.0 & No & No & No & No & Infinite & 0.2822 & 83.4 \\
\hline 5.0 & Yes & No & No & No & Infinite & 0.8916 & 0.248 \\
\hline 5.0 & No & Yes & No & No & Infinite & 0.2329 & 74.6 \\
\hline 5.0 & No & No & Yes & No & Infinite & 0.2007 & 174.8 \\
\hline 5.0 & No & No & No & Yes & Infinite & 0.2417 & 105.0 \\
\hline 5.0 & Yes & Yes & No & No & Infinite & 0.8560 & 0.237 \\
\hline 5.0 & Yes & No & Yes & No & Infinite & 0.8353 & 0.255 \\
\hline 5.0 & Yes & No & No & Yes & Infinite & 0.8623 & 0.253 \\
\hline 5.0 & Yes & Yes & Yes & Yes & Infinite & 0.8525 & 0.239 \\
\hline 5.0 & Yes & No & No & Yes $(0.05)$ & Infinite & 0.8884 & 0.248 \\
\hline 5.0 & Yes & No & No & Yes $(0.1)$ & Infinite & 0.8857 & 0.248 \\
\hline 8.0 & No & No & No & No & Infinite & 0.3310 & 88.1 \\
\hline 8.0 & Yes & No & No & No & Infinite & 0.9548 & 0.375 \\
\hline 8.0 & No & Yes & No & No & Infinite & 0.2674 & 81.0 \\
\hline 8.0 & No & No & Yes & No & Infinite & 0.2310 & 175.2 \\
\hline 8.0 & No & No & No & Yes & Infinite & 0.2805 & 108.3 \\
\hline 8.0 & Yes & Yes & No & No & Infinite & 0.9187 & 0.351 \\
\hline 8.0 & Yes & No & Yes & No & Infinite & 0.8960 & 0.378 \\
\hline 8.0 & Yes & No & No & Yes & Infinite & 0.9250 & 0.380 \\
\hline 8.0 & Yes & Yes & Yes & Yes & Infinite & 0.9152 & 0.352 \\
\hline 8.0 & Yes & No & No & Yes $(0.05)$ & Infinite & 0.9523 & 0.377 \\
\hline 8.0 & Yes & No & No & Yes $(0.1)$ & Infinite & 0.9492 & 0.376 \\
\hline $8.0^{2}$ & Yes & No & No & Yes & Single & 0.8876 & 0.386 \\
\hline $8.0^{2}$ & Yes & Yes & No & Yes & Single & 0.9147 & 0.354 \\
\hline $8.0^{2}$ & Yes & Yes & Yes & Yes & Single & 0.9141 & 0.351 \\
\hline $8.0^{3}$ & Yes & No & No & No & Infinite & 0.8983 & 0.432 \\
\hline
\end{tabular}

${ }^{1}$ Includes fuel pellet-clad gap

${ }^{2}$ Single package $(\mathrm{N}=0.5$, criticality safety index $=100)$

${ }^{3}$ Fuel includes 52 IFBA rods

Replacing 52 fuel rods with the IFBA rods included in the $8 \mathrm{wt} . \%$ fuel assembly lattice produced a maximum $k_{\text {eff }}$ similar to the $5 \mathrm{wt} . \%$ base case. Reducing the array size of the $8 \mathrm{wt} . \%$ case to a single package ( $20 \mathrm{~cm}$ water reflector with vacuum boundary conditions) reduced $k_{\text {eff }}$ by 0.03 , which was insufficient to reach the target. Nevertheless, the $8 \mathrm{wt} \%$ single package case was used to evaluate cross section $\mathrm{S} / \mathrm{U}$ and similarity to benchmark critical experiments. The single package case $k_{\text {eff }}$ was maximized with Clamshell, Outerpack, and external flooding.

The single package case $k_{\text {eff }}$ was maximized with Clamshell, fuel lattice, pellet-clad gap region, Outerpack, and external flooding. The $5 \mathrm{wt} \%$ base case, 8 wt.\% 52 IFBA case, and 8 wt.\% single package case highlighted in Table 3 were used in the TSUNAMI calculations.

Table 4 contains additional cases to provide enrichment and array size $k_{\text {eff }}$ sensitivity. Enrichment sensitivity is approximately $0.02 \Delta \mathrm{k} / \mathrm{wt} . \%{ }^{235} \mathrm{U}$. Sensitivity to HAC array size is highly nonlinear. The total reduction in $k_{\text {eff }}$ for a single package relative to an infinite array is approximately $0.04 \Delta \mathrm{k}$.

Determining a trade-off between array size and enrichment for the Traveller is complex because as currently licensed, Traveller criticality safety index (CSI) values range from 0.7 to 4.2 (equivalent to analyzed HAC array sizes of 12 to 72) [6]. With the Table 4 results, a maximum $k_{\text {eff }}$ margin of roughly 
$0.03 \Delta \mathrm{k}$ could be gained by increasing the CSI to 50 (equivalent to $1.5 \%$ enrichment increase). The Traveller safety analysis also shows that the Traveller STD is less reactive than the XL version by 0.01$0.02 \Delta \mathrm{k}$, which could allow a higher enrichment limit for the STD version. Explicitly modeling the set of limiting conditions for each fuel design and package version could also provide some margin relative to the bounding design and bounding penalty method.

Table 4. Traveller fuel assembly enrichment and array size sensitivity cases.

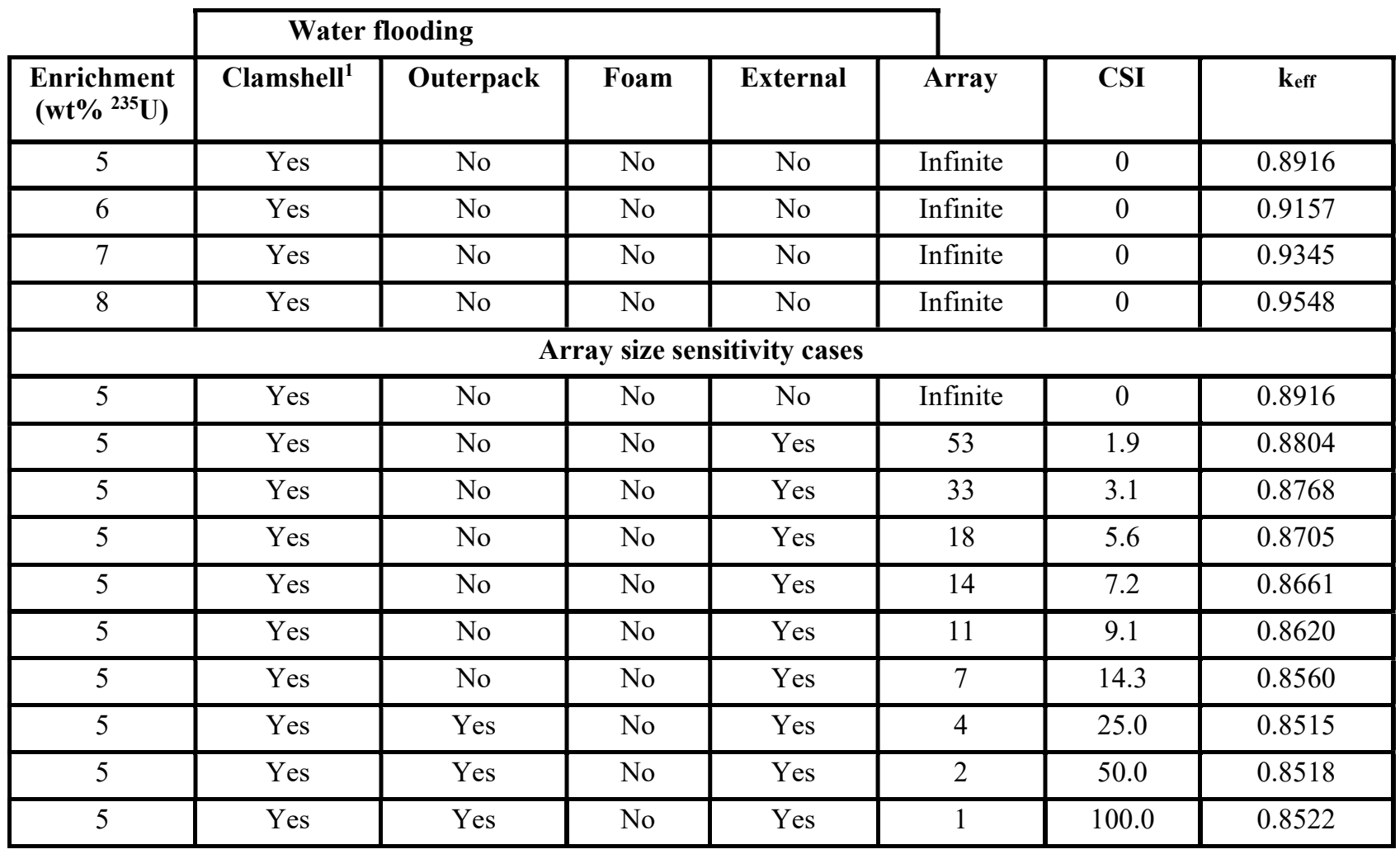

$\mathrm{CSI}=50 / \mathrm{N}$ rounded up to the nearest 0.1 , where $\mathrm{N}=$ analyzed $\mathrm{HAC}$ array size $/ 2$ 


\subsection{Traveller Fuel Assembly Model TSUNAMI-3D Calculations}

TSUNAMI-3D S/U calculations were performed for the three highlighted cases from Table 3. Table 5 shows key TSUNAMI input values, forward and adjoint $k_{\text {eff, }}$ the energy of the average lethargy of fission, and total cross section uncertainty.

Table 5. Traveller fuel assembly model TSUNAMI-3D case data.

\begin{tabular}{|c|c|c|c|}
\hline Parameter & 5 wt.\% infinite array & $\mathbf{8 ~ w t . \% ~ s i n g l e ~ p a c k a g e ~}$ & 8 wt.\% 52 IFBA infinite array \\
\hline Grid mesh & $40 \times 42 \times 44$ & $44 \times 44 \times 58$ & $40 \times 42 \times 44$ \\
\hline Particles/gen. & $10,000 \mathrm{fwd}$. $/ 30,000 \mathrm{adj}$. & $10,000 \mathrm{fwd} . / 30,000 \mathrm{adj}$. & $10,000 \mathrm{fwd} . / 30,000 \mathrm{adj}$. \\
\hline SDF file name & T3D_Trav_FA_wet1_mm2 & $\begin{array}{c}\text { T3D_Trav_FA8_wet2a_single_ } \\
\text { axial2 }\end{array}$ & T3D_Trav_FA8I_wet1_mm \\
\hline Forward $k_{\text {eff }}$ & $0.89139 \pm 0.00034$ & $0.91447 \pm 0.00028$ & $0.89772 \pm 0.00034$ \\
\hline Adjoint $k_{\text {eff }}$ & $0.89098 \pm 0.00099$ & $0.91425 \pm 0.00089$ & $0.89868 \pm 0.00099$ \\
\hline EALF & $0.247 \mathrm{eV}$ & $0.350 \mathrm{eV}$ & $0.433 \mathrm{eV}$ \\
\hline $\begin{array}{c}\text { Cross section } \\
\text { uncertainty }\end{array}$ & $0.671 \% \Delta \mathrm{k} / \mathrm{k}$ & $0.877 \% \Delta \mathrm{k} / \mathrm{k}$ & $0.633 \% \Delta \mathrm{k} / \mathrm{k}$ \\
\hline
\end{tabular}

TSUNAMI input adequacy was confirmed by comparing TSUNAMI-calculated nuclide sensitivity with KENO direct perturbation (DP) sensitivity. Figures 5, 6, and 7 summarize the sensitivity results calculated by each method for the highest sensitivity nuclide/region combinations. There is good agreement between the DP $(\% \Delta \mathrm{k} / \% \rho)$ and TSUNAMI $(\% \Delta \mathrm{k} / \% \Sigma)$ sensitivities, which confirms that the TSUNAMI SDFs properly represent the models.

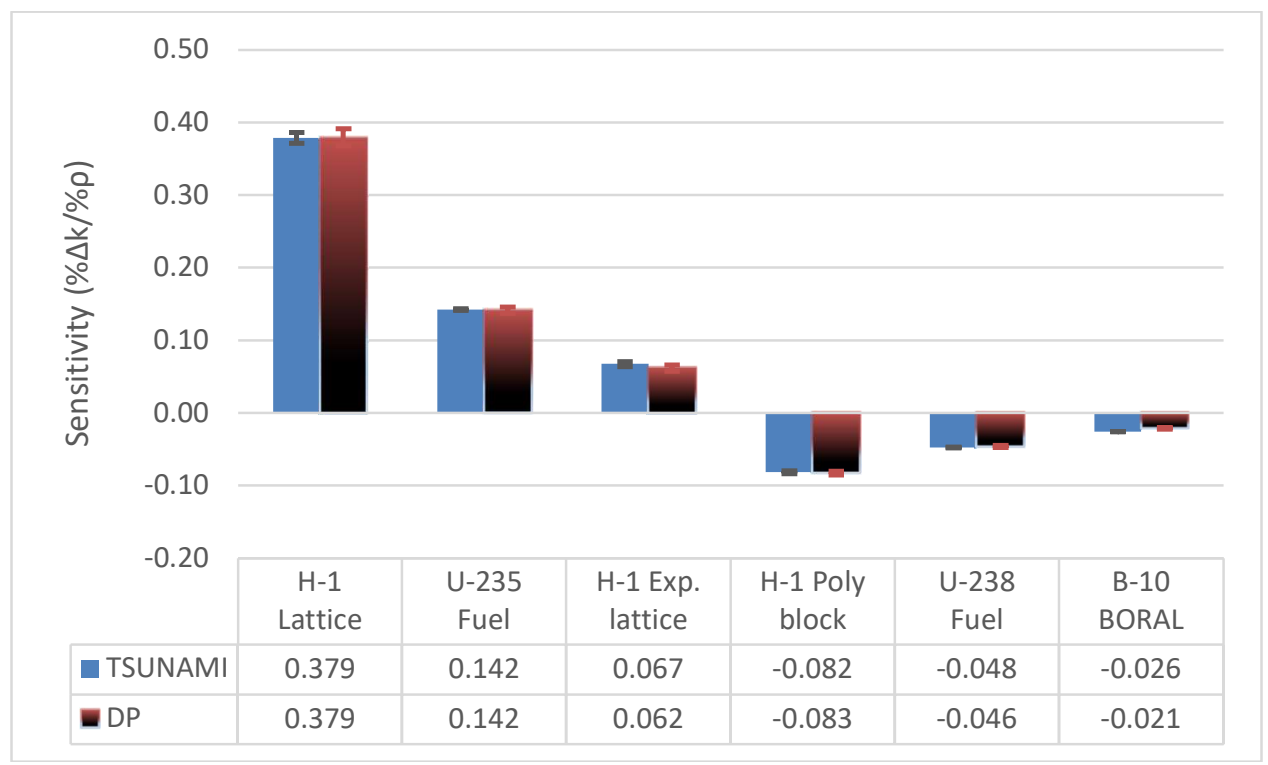

Figure 5. Sensitivity of the infinite array of $5 \mathrm{wt} \%$ fuel assembly packages. 


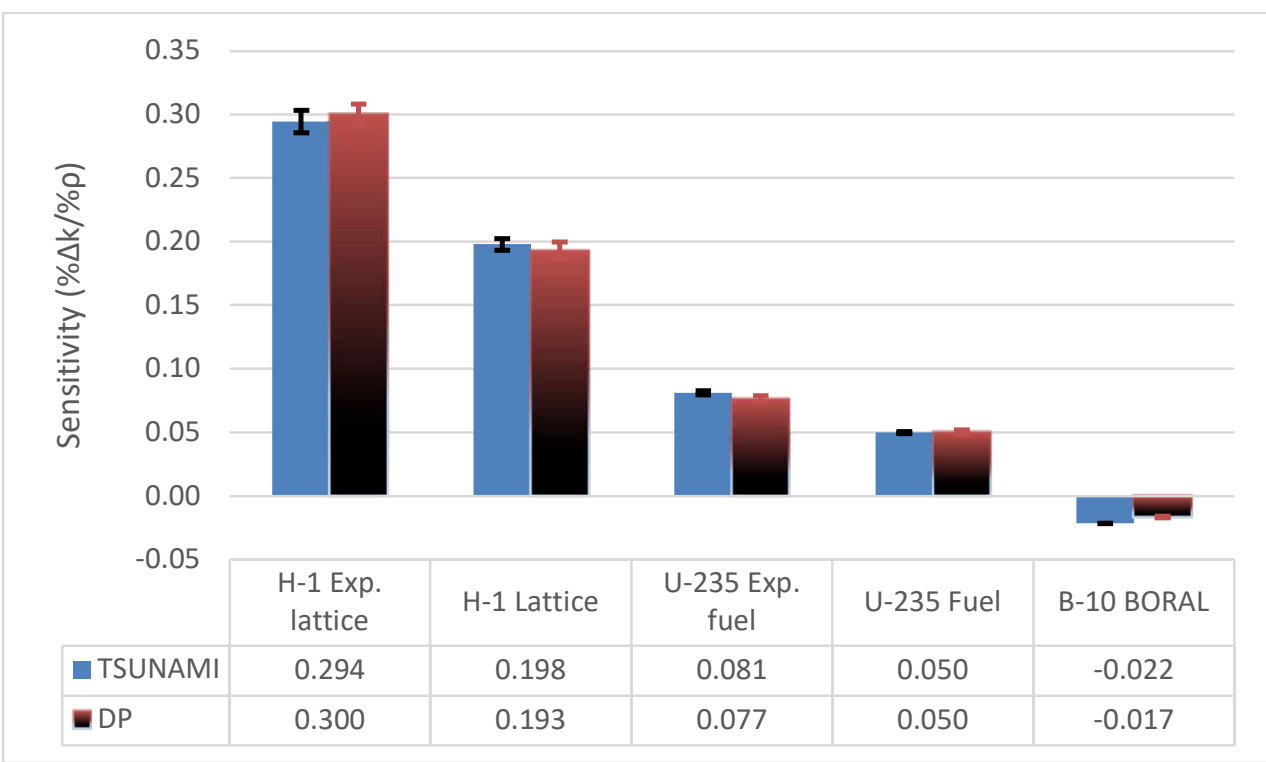

Figure 6. Sensitivity of the single package model with an $8 \mathrm{wt} . \%$ fuel assembly.

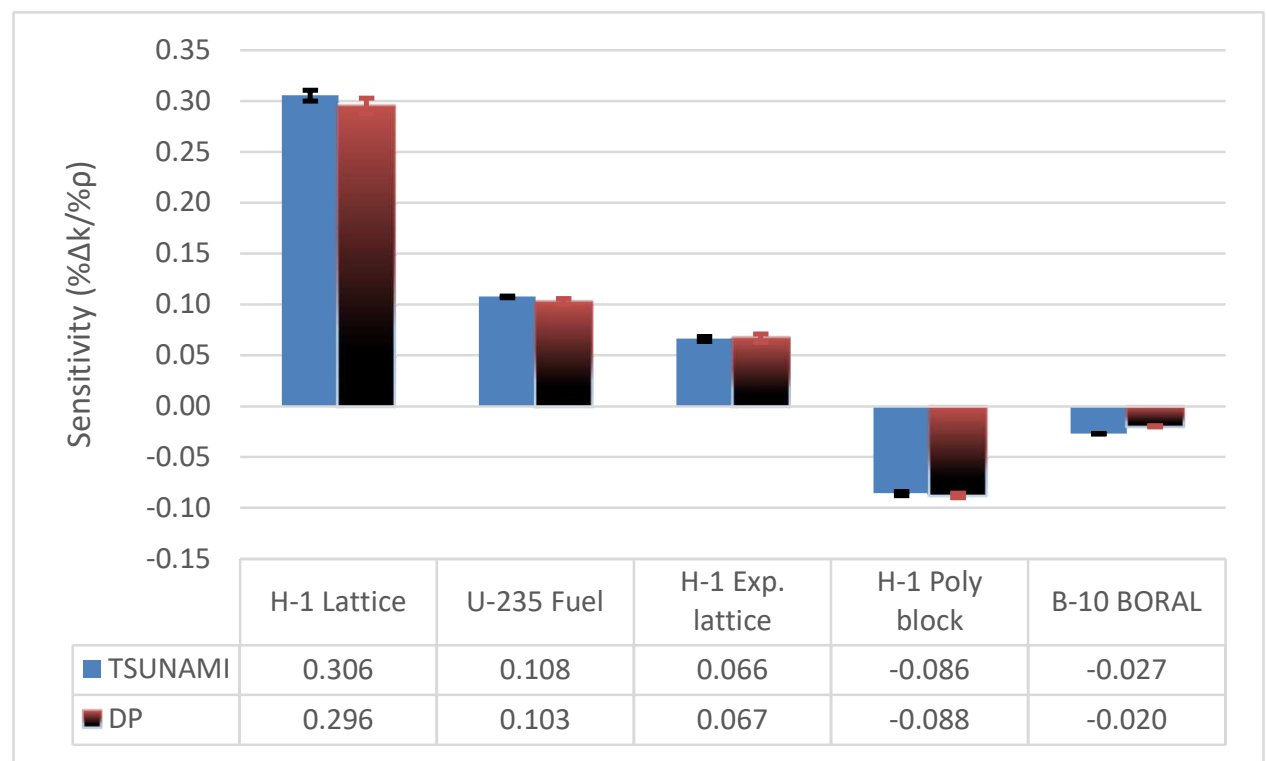

Figure 7. Sensitivity of the infinite array of 8 wt.\% 52 IFBA rod fuel assembly packages.

Sensitivity results provided insight into which materials and regions are most important for criticality control. For example, in the infinite array models (Figures 4 and 6), $k_{\text {eff }}$ is most sensitive to $\mathrm{H}$ in the regular fuel lattice water (increasing ${ }^{1} \mathrm{H}$ increases $k_{\text {eff }}$ ). In the single package model (Figure 5 ), $k_{\text {eff }}$ is most sensitive to $\mathrm{H}$ in the expanded pitch fuel lattice water (increasing ${ }^{1} \mathrm{H}$ increases $k_{\text {eff }}$ ).

Table 6 lists the sensitivity for each mixture in each model. Values greater than $0.01 \% \Delta \mathrm{k} / \% \Sigma$ are highlighted. The two infinite array models (5 wt.\% and $8 \mathrm{wt} . \%$ with 52 IFBA rods) have similar sensitivities. Materials with the highest positive sensitivity ( $k_{\text {eff }}$ increases with density) are water in the fuel lattice and $\mathrm{UO}_{2}$. Polyethylene blocks and Boral have the most significant negative sensitivities. The biggest difference between the infinite package array models is that sensitivity is split between IFBA and non-IFBA fuel lattice materials. In contrast to the infinite package array models, the $8 \mathrm{wt} . \%$ single package model has: (1) higher sensitivity in the expanded lattice region than in the physically larger 
regular lattice region, (2) small positive sensitivity to water in the overpack, and (3) almost no sensitivity to the polyethylene blocks. The latter two observations are explained by recognizing that water in the overpack in package arrays increases moderation but tends to reduce interaction between packages, whereas water in the overpack in a single package only increases moderation.

Table 6. Traveller fuel assembly model sensitivity by mixture.

\begin{tabular}{|c|c|c|c|c|c|c|c|}
\hline \multirow[b]{2}{*}{ Mixture } & \multirow[b]{2}{*}{ Description } & \multicolumn{2}{|c|}{$\begin{array}{c}5 \text { wt\% } 17 x 17 \text { Inf. } \\
\text { Array }\end{array}$} & \multicolumn{2}{|c|}{8 wt\% $17 \times 17$ Single } & \multicolumn{2}{|c|}{$\begin{array}{c}8 \text { wt\% 17x17 IFBA } \\
\text { Inf. Array }\end{array}$} \\
\hline & & Sensitivity & Std. Dev. & Sensitivity & Std. Dev. & Sensitivity & Std. Dev. \\
\hline 1 & $\mathrm{UO}_{2}(\mathrm{NL})$ & 0.114 & $0.6 \%$ & 0.051 & $0.9 \%$ & 0.101 & $0.6 \%$ \\
\hline 2 & Fuel clad (NL) & 0.007 & $1.1 \%$ & 0.005 & $0.9 \%$ & 0.006 & $1.0 \%$ \\
\hline 3 & Fuel lattice water (NL) & 0.413 & $0.9 \%$ & 0.218 & $1.1 \%$ & 0.333 & $0.8 \%$ \\
\hline 4 & SS structure & -0.006 & $1.0 \%$ & 0.000 & $7.2 \%$ & -0.006 & $1.0 \%$ \\
\hline 5 & BORAL & -0.023 & $0.3 \%$ & -0.018 & $0.4 \%$ & -0.024 & $0.3 \%$ \\
\hline 6 & Clamshell water & 0.030 & $2.0 \%$ & 0.018 & $2.7 \%$ & 0.028 & $2.1 \%$ \\
\hline 7 & Clamshell aluminum & 0.004 & $1.2 \%$ & 0.009 & $0.6 \%$ & 0.004 & $1.2 \%$ \\
\hline 8 & Polyethylene blocks & -0.078 & $1.2 \%$ & 0.001 & $79.6 \%$ & -0.083 & $1.3 \%$ \\
\hline 10 & Polyethylene packaging & 0.004 & $3.6 \%$ & 0.005 & $5.3 \%$ & 0.003 & $4.1 \%$ \\
\hline 13 & Fuel-clad gap water (NL) & 0.005 & $7.1 \%$ & 0.002 & $9.5 \%$ & 0.004 & $6.2 \%$ \\
\hline 14 & Water in outerpack & 0.000 & $0.7 \%$ & 0.010 & $11.4 \%$ & 0.000 & $0.7 \%$ \\
\hline 15 & Water in outerpack foam & 0.000 & $0.4 \%$ & 0.000 & $0.4 \%$ & 0.000 & $0.4 \%$ \\
\hline 16 & Water reflector & 0.000 & $0.8 \%$ & 0.000 & $17.4 \%$ & 0.000 & $0.8 \%$ \\
\hline 21 & $\mathrm{UO}_{2}(\mathrm{EL})$ & 0.023 & $1.2 \%$ & 0.085 & $1.0 \%$ & 0.018 & $1.3 \%$ \\
\hline 22 & Fuel clad (EL) & 0.001 & $2.3 \%$ & 0.008 & $1.2 \%$ & 0.001 & $2.4 \%$ \\
\hline 23 & Fuel lattice water (EL) & 0.075 & $2.2 \%$ & 0.329 & $1.3 \%$ & 0.054 & $2.1 \%$ \\
\hline 33 & Fuel-clad gap water (EL) & 0.001 & $19.3 \%$ & 0.001 & $11.4 \%$ & 0.000 & $17.1 \%$ \\
\hline 101 & $\mathrm{UO} 2$ (INL) & & & & & 0.035 & $0.9 \%$ \\
\hline 102 & Fuel clad (INL) & & & & & 0.001 & $3.4 \%$ \\
\hline 103 & Fuel lattice water (INL) & & & & & 0.071 & $1.7 \%$ \\
\hline 113 & Fuel-clad gap water (INL) & & & & & 0.001 & $16.4 \%$ \\
\hline 114 & IFBA (INL) & & & & & -0.005 & $1.4 \%$ \\
\hline 121 & UO2 (IEL) & & & & & 0.006 & $2.1 \%$ \\
\hline 122 & Fuel clad (IEL) & & & & & 0.000 & $8.2 \%$ \\
\hline 123 & Fuel lattice water (IEL) & & & & & 0.012 & $4.5 \%$ \\
\hline 124 & Fuel-clad gap water (IEL) & & & & & -0.001 & $4.4 \%$ \\
\hline 133 & IFBA (IEL) & & & & & 0.000 & $50.2 \%$ \\
\hline
\end{tabular}

Region codes: NL (normal fuel lattice), EL (expanded fuel lattice), INL (IFBA pin in normal fuel lattice), IEL (IFBA pin in expanded fuel lattice) 


\subsection{Traveller Fuel Assembly Model TSUNAMI-IP Results}

TSUNAMI-IP was used to calculate correlation coefficients $\left(c_{k}\right)$ for each of the three SDF files in Table 4 and the library of 1,584 critical experiment SDF files. Figures 8, 9, and 10 show the results with different symbols representing different types of experiments. IEU includes all intermediate enrichment experiments, LEU includes low enrichment experiments not captured by the other categories, LCT includes LEU-COMP-THERM experiments, LMP includes LEU-MET-THERM experiments, and LST includes LEU-SOL-THERM experiments. Table 7 shows the number of experiments in each category with $c_{k} \geq 0.9$ and the median $c_{k}$ of the 1,584 experiments for each of the three Traveller models. A full list of experiments with $c_{k}$ values is provided in Appendix A. The TSUNAMI results show that many experiments are likely to be applicable for code validation for the Traveller PWR fuel assembly models with $\mathrm{UO}_{2}$ fuel enrichment up to $8 \mathrm{wt} . \%{ }^{235} \mathrm{U}$.

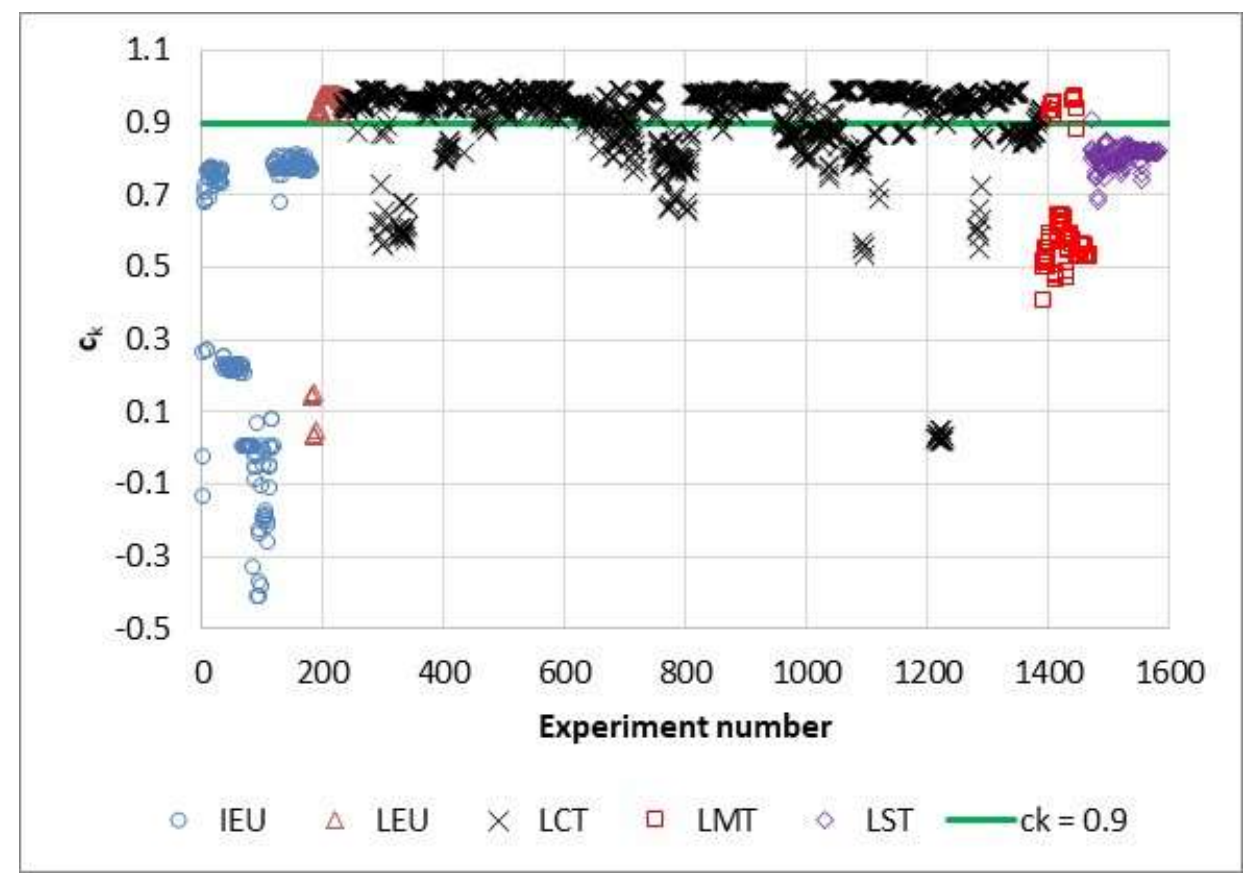

Figure 8. Traveller $c_{k}$ for the infinite array of packages with $5 \mathrm{wt} \%$ fuel assemblies. 


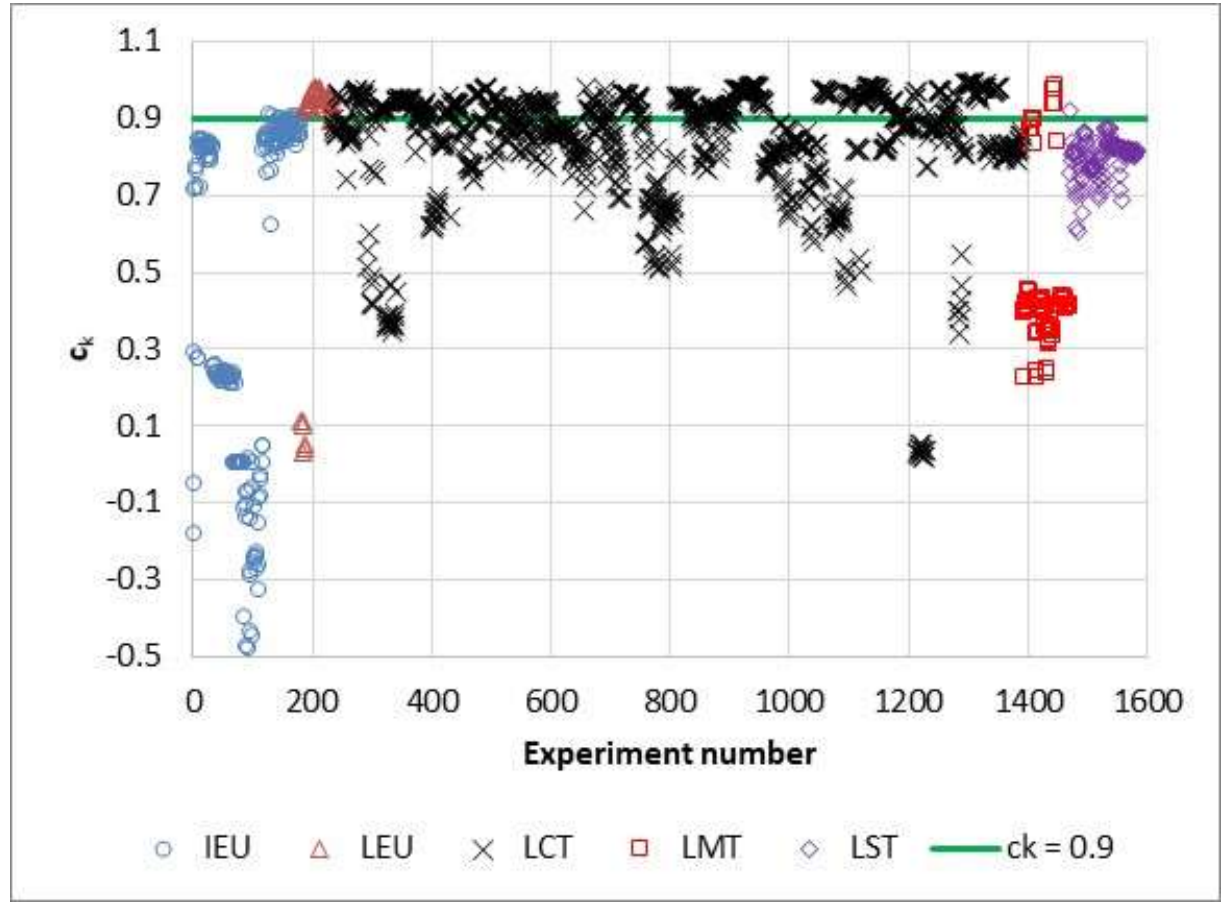

Figure 9. Traveller $c_{k}$ for a single package with an $8 \mathrm{wt} . \%$ fuel assembly.

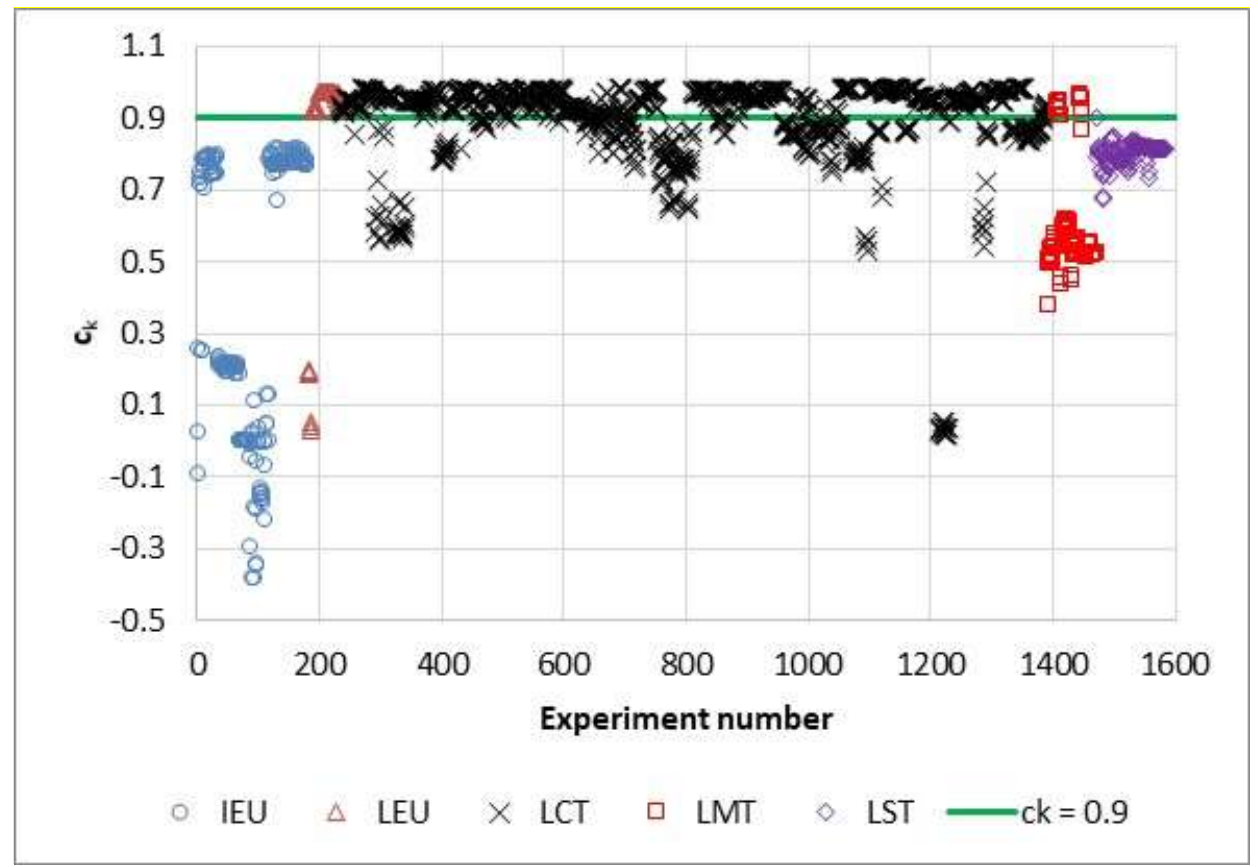

Figure 10. Traveller $c_{k}$ for the infinite array of packages with $8 \mathrm{wt} . \% 52$ IFBA rod fuel assemblies. 
Table 7. Traveller fuel assembly model TSUNAMI-IP similarity summary.

\begin{tabular}{|c|c|c|c|}
\cline { 2 - 4 } \multicolumn{1}{c|}{} & \multicolumn{3}{c|}{ Experiments with $\boldsymbol{c}_{\boldsymbol{k}} \geq \mathbf{0 . 9}$} \\
\hline Category & $\mathbf{5}$ wt.\% array & 8 wt.\% single & 8 wt.\% 52 IFBA array \\
\hline IEU $c_{k} \geq 0.9$ & 0 & 5 & 0 \\
LEU $c_{k} \geq 0.9$ & 48 & 47 & 48 \\
\hline LCT $c_{k} \geq 0.9$ & 830 & 528 & 801 \\
\hline LMT $c_{k} \geq 0.9$ & 13 & 7 & 13 \\
\hline LST $c_{k} \geq 0.9$ & 1 & 1 & 1 \\
\hline Median $c_{k}$ & 0.931 & 0.864 & 0.921 \\
\hline
\end{tabular}

\subsection{Traveller Rod Pipe Model Criticality Calculations}

The Traveller package was also used to transport fuel rods in a rod pipe. To cover potential combinations of fuel pellet outer diameters (OD) and the number of rods in the pipe, Sampler was used to direct KENO infinite array calculations for 11 pellet OD and 11 fuel rod pitch values (121 cases). Pellet OD ranged from 0.78 to $1.3 \mathrm{~cm}$. Rod pitch varied from 0.1 to $1.0 \mathrm{~cm}$ larger than the pellet OD. No fuel clad is modeled, polyethylene moderator fills the non-fuel space in the rod pipe, and water flooding is assumed in the Clamshell. Other regions are dry.

Sampler/KENO $k_{\text {eff }}$ results for $5 \mathrm{wt} . \% \mathrm{UO}_{2}$ fuel rods are shown in Figure 11. Maximum reactivity occurs with pellet $\mathrm{OD} 0.78 \mathrm{~cm}$ and $1.42 \mathrm{~cm}$ rod pitch. Results for $10 \mathrm{wt} \% \mathrm{UO}_{2}$ fuel rods are shown in Figure 12. Maximum reactivity occurs with pellet OD $0.884 \mathrm{~cm}$ and $1.70 \mathrm{~cm}$ rod pitch.

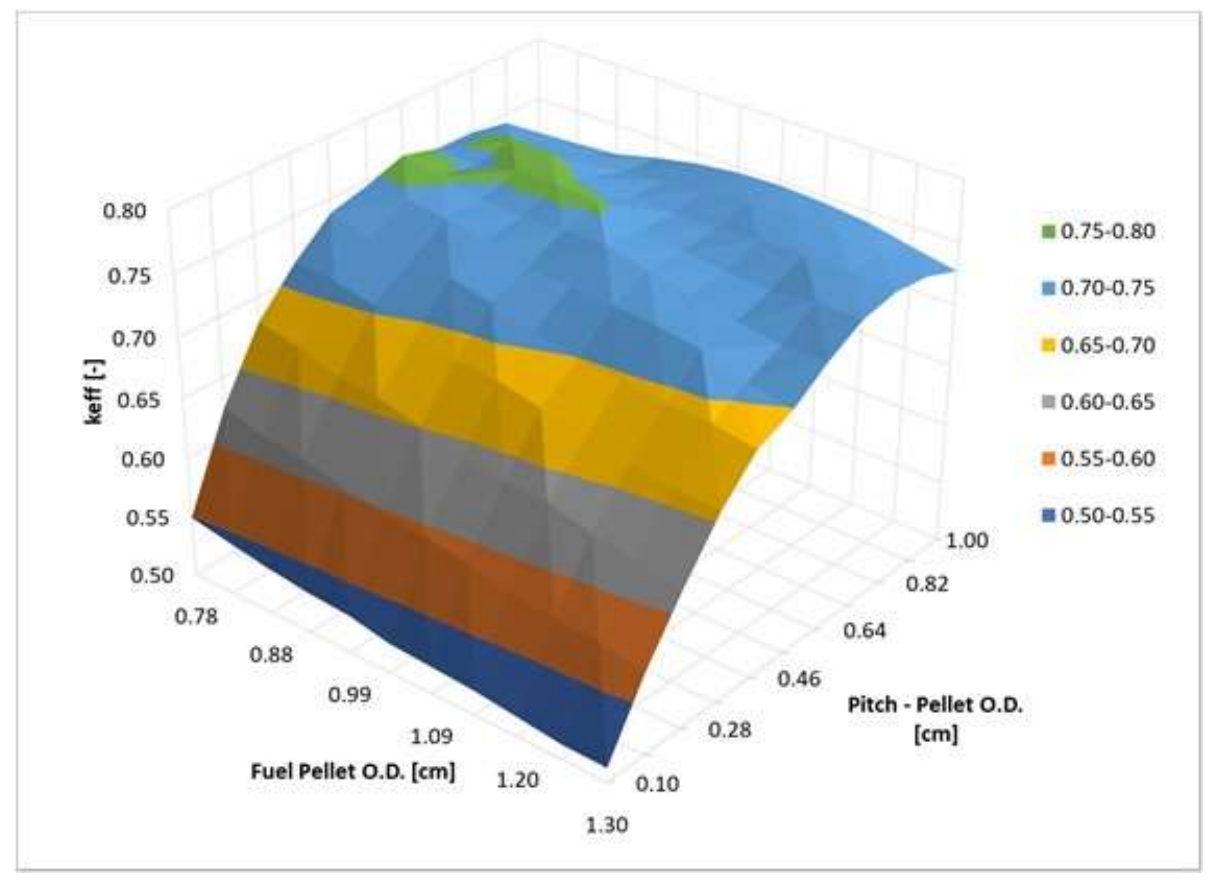

Figure 11. Rod pipe model $k_{e f f}$ for fuel pellet OD and rod pitch combinations for $5 \mathrm{wt} . \% \mathrm{UO}_{2}$. 


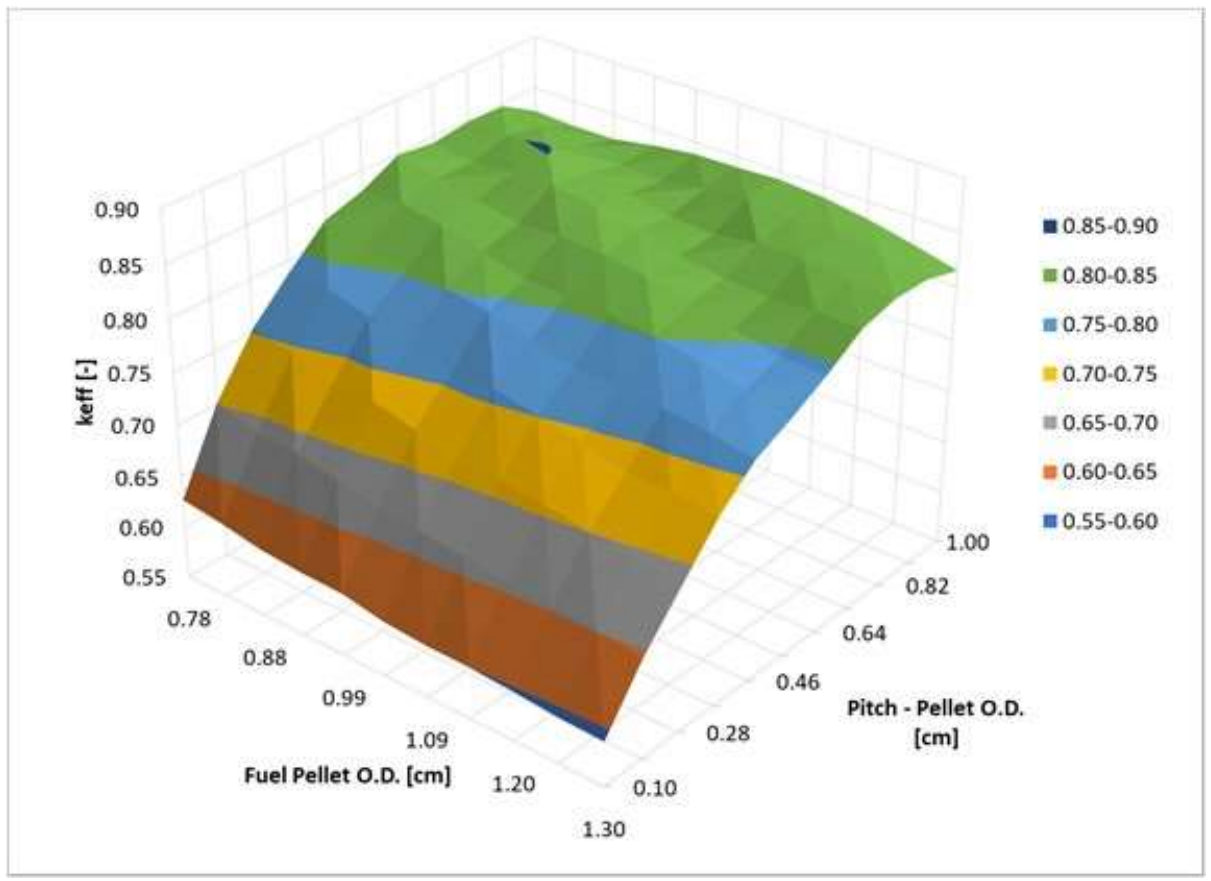

Figure 12. Rod pipe model $k_{\text {eff }}$ for fuel pellet OD and rod pitch combinations for 10 wt. $\% \mathrm{UO}_{2}$.

Additional preferential flooding cases (i.e., overpack, foam, and external) were run by using the Sampler maximum reactivity cases. The flooding cases confirmed that the assumed Clamshell-only flooding produces the highest $k_{\text {eff. }}$. With $10 \mathrm{wt} . \% \mathrm{UO}_{2}$ fuel rods, the Traveller rod pipe $k_{\text {eff }}(0.851)$ is substantially less than the base case target (0.892). This means that the Traveller package in its current form can likely support the transportation of $\mathrm{UO}_{2}$ fuel rods up to $10 \mathrm{wt} . \%{ }^{235} \mathrm{U}$.

\subsection{Traveller Rod Pipe Model TSUNAMI-3D Calculations}

TSUNAMI-3D S/U calculations were performed for the 5 and $10 \mathrm{wt} . \%$ most reactive rod pipe cases.

Table 8 shows key TSUNAMI input values, forward and adjoint $k_{\text {eff, }}$, the energy of the average lethargy of fission, and total cross section uncertainty.

Table 8. Traveller rod pipe model TSUNAMI-3D case data.

\begin{tabular}{|c|c|c|}
\hline Parameter & 5 wt.\% infinite array & 10 wt.\% infinite array \\
\hline Grid mesh & $40 \times 42 \times 44$ & $40 \times 42 \times 44$ \\
\hline Particles/gen. & $10,000 \mathrm{fwd} . / 30,000 \mathrm{adj}$. & $10,000 \mathrm{fwd} . / 30,000 \mathrm{adj}$. \\
\hline SDF file & T3D_Trav_RP5 & T3D_Trav_RP10 \\
\hline Forward $k_{\text {eff }}$ & $0.75819 \pm 0.00049$ & $0.84951 \pm 0.00049$ \\
\hline Adjoint $k_{\text {eff }}$ & $0.75800 \pm 0.0014$ & $0.8491 \pm 0.0014$ \\
\hline EALF & $0.181 \mathrm{eV}$ & $0.255 \mathrm{eV}$ \\
\hline $\begin{array}{c}\text { Cross section } \\
\text { uncertainty }\end{array}$ & $0.904 \% \Delta \mathrm{k} / \mathrm{k}$ & $0.880 \% \Delta \mathrm{k} / \mathrm{k}$ \\
\hline
\end{tabular}

Figures 13 and 14 summarize the sensitivity results calculated by TSUNAMI and DP KENO cases for the highest sensitivity nuclide/region combinations. There is good agreement between the DP and TSUNAMI sensitivities, which confirms that the TSUNAMI SDFs properly represent the models. The results also provide insight into what materials and regions are most important for criticality control. For example, in each model, $k_{\text {eff }}$ is most sensitive to $\mathrm{H}$ in the fuel lattice region polyethylene (increasing ${ }^{1} \mathrm{H}$ increases $k_{\text {eff }}$ ). 


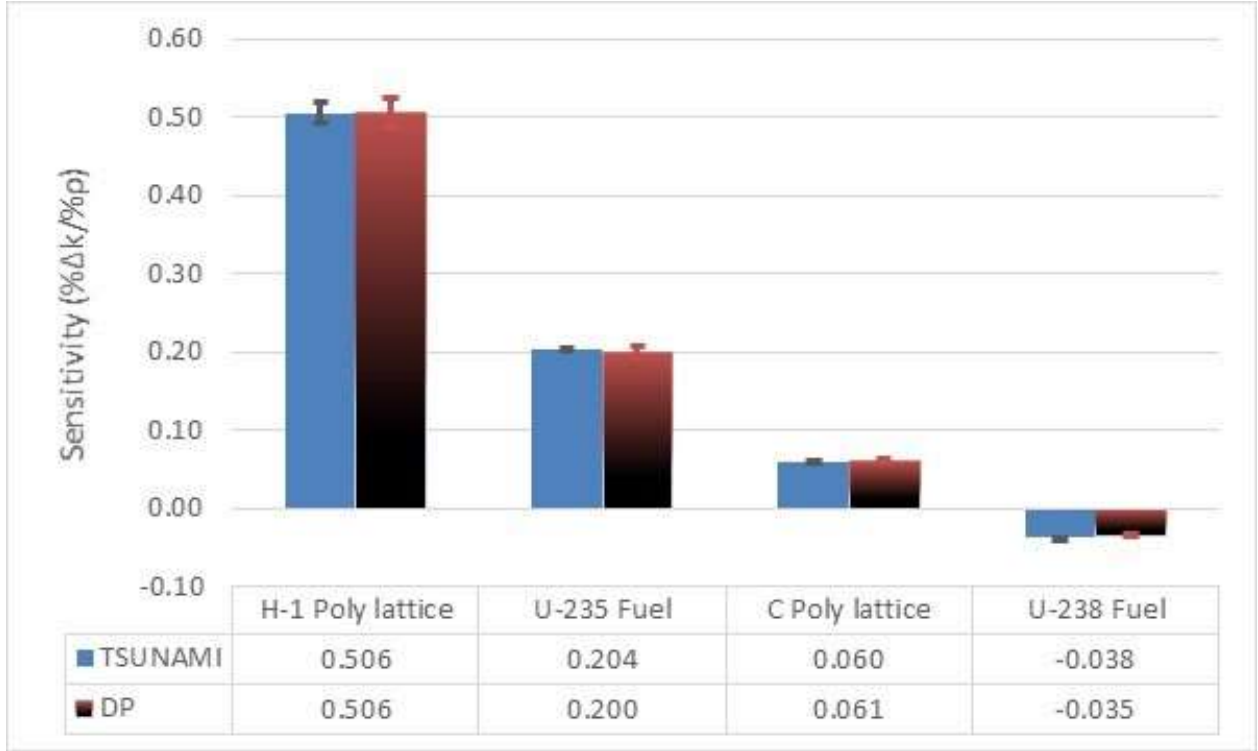

Figure 13. Sensitivity of the infinite array of packages with $5 \mathrm{wt} . \% \mathrm{UO}_{2}$ fuel rods.

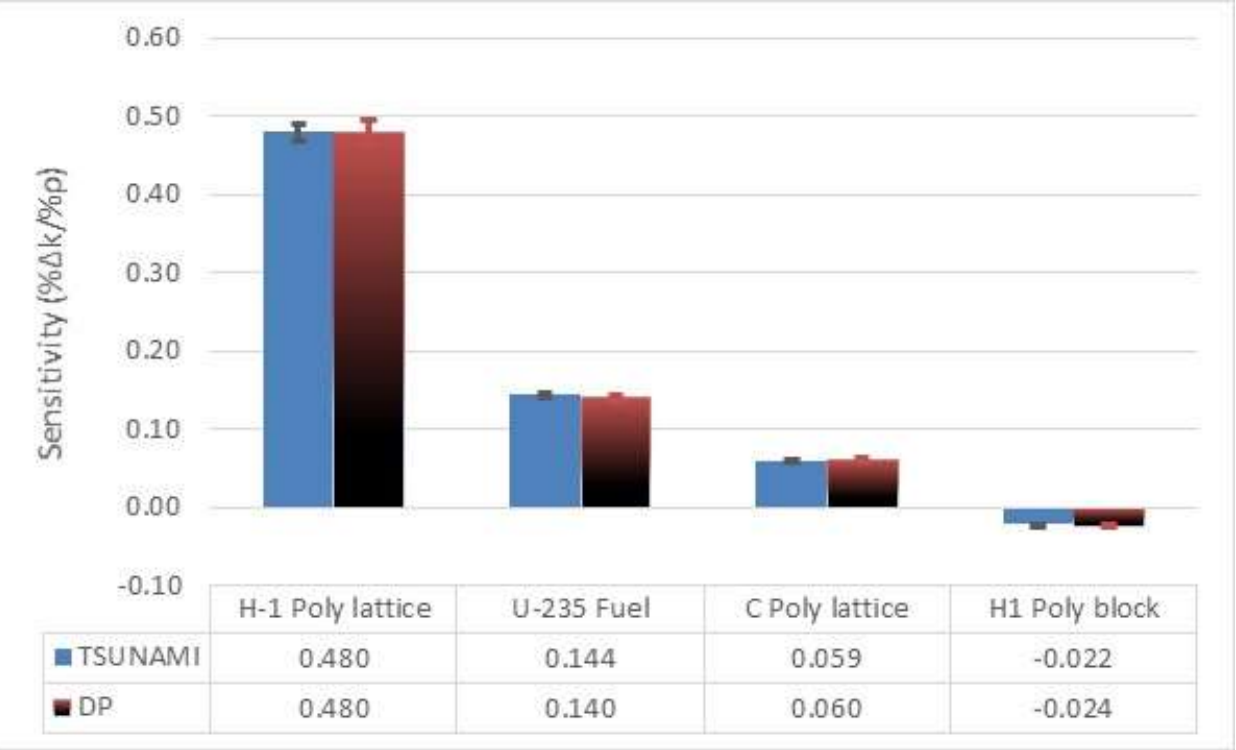

Figure 14. Sensitivity of the infinite array of packages with $10 \mathrm{wt} . \% \mathrm{UO}_{2}$ fuel rods.

Table 9 lists the sensitivity for each mixture in each model. Values greater than $0.01 \% \Delta \mathrm{k} / \% \Sigma$ are highlighted. The two rod pipe models have very similar mixture sensitivities. One interesting feature is that Boral is unimportant for criticality control as compared with SS shells or polyethylene blocks. This is probably due to the SS shells and polyethylene blocks reducing package-to-package neutron interaction in the package array. 
Table 9. Traveller rod pipe model sensitivity by mixture.

\begin{tabular}{|c|c|c|c|c|c|}
\hline \multirow[b]{2}{*}{ Mixture } & \multirow[b]{2}{*}{ Description } & \multicolumn{2}{|c|}{5 wt. \% rod pipe } & \multicolumn{2}{|c|}{10 wt. $\%$ rod pipe } \\
\hline & & Sensitivity & \% std. dev. & Sensitivity & $\%$ std. dev. \\
\hline 1 & $\mathrm{UO}_{2}$ & 0.190 & $0.6 \%$ & 0.150 & $0.7 \%$ \\
\hline 3 & Fuel lattice polyethylene & 0.567 & $1.3 \%$ & 0.539 & $1.1 \%$ \\
\hline 4 & SS structure & -0.018 & $2.2 \%$ & -0.014 & $2.4 \%$ \\
\hline 5 & Boral & -0.002 & $1.9 \%$ & -0.002 & $1.7 \%$ \\
\hline 6 & Clamshell water & 0.053 & $6.1 \%$ & 0.051 & $5.9 \%$ \\
\hline 7 & Clamshell Al & 0.001 & $2.6 \%$ & 0.001 & $2.4 \%$ \\
\hline 8 & Polyethylene blocks & -0.021 & $1.2 \%$ & -0.021 & $1.1 \%$ \\
\hline 14 & Water in Outerpack & 0.000 & $1.0 \%$ & 0.000 & $0.9 \%$ \\
\hline 15 & Water in Outerpack foam & 0.000 & $0.5 \%$ & 0.000 & $0.4 \%$ \\
\hline 16 & Water reflector & 0.000 & $0.9 \%$ & 0.000 & $0.8 \%$ \\
\hline
\end{tabular}

\subsection{Traveller Rod Pipe Model TSUNAMI-IP Results}

TSUNAMI-IP was used to calculate correlation coefficients $\left(c_{k}\right)$ for the two SDF files in Table 7 and the library of 1,584 critical experiment SDF files. Figures 15 and 16 show the results with different symbols representing different types of experiments. Table 10 shows the number of experiments in each category with $c_{k} \geq 0.9$ for the two Traveller rod pipe models. A full list of experiments with $c_{k}$ values is provided in Appendix A. The TSUNAMI results show that many experiments are applicable for code validation for Traveller rod pipe models with $\mathrm{UO}_{2}$ fuel enrichment up to $10 \mathrm{wt} . \%{ }^{235} \mathrm{U}$.

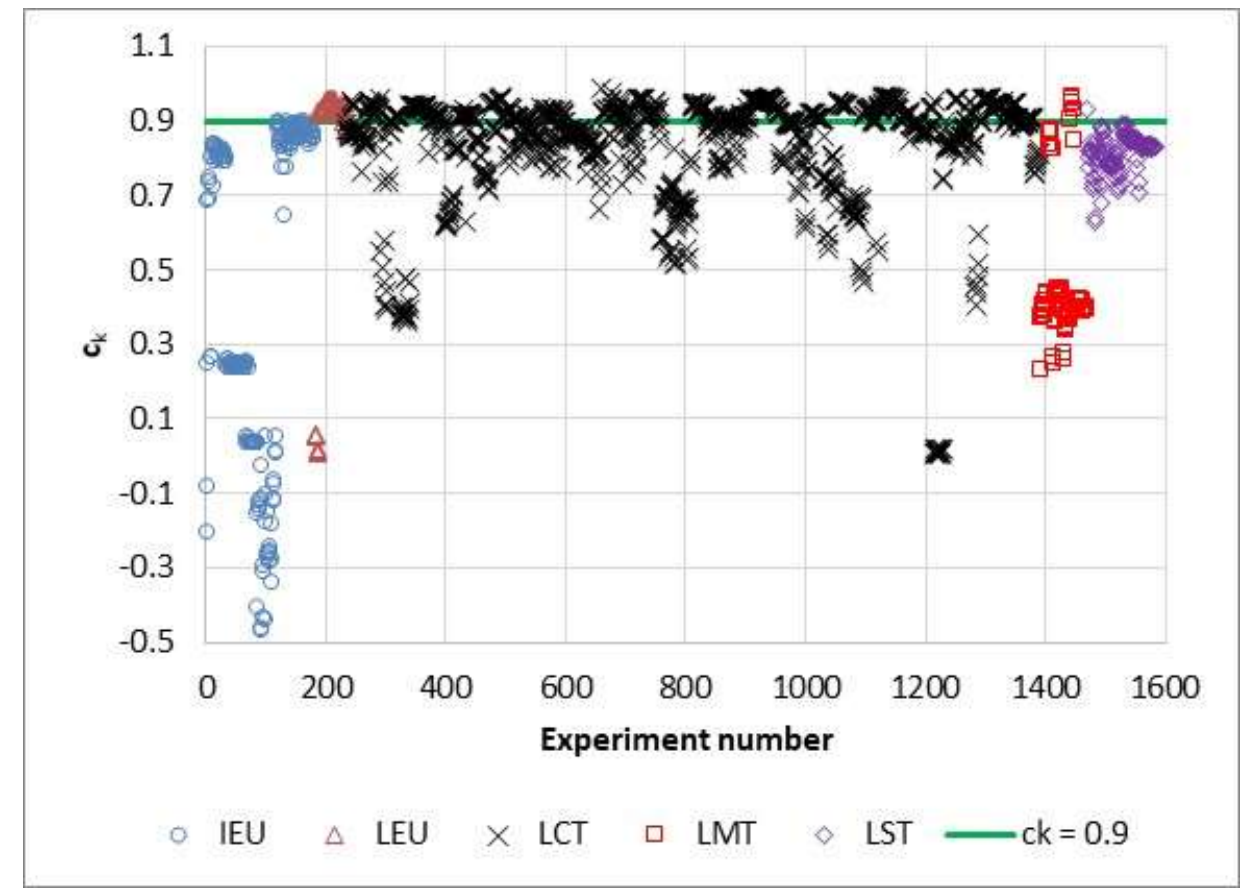

Figure 15. Traveller $c_{k}$ for the infinite array of packages with $5 \mathrm{wt} \% \mathrm{UO}_{2}$ fuel rods. 


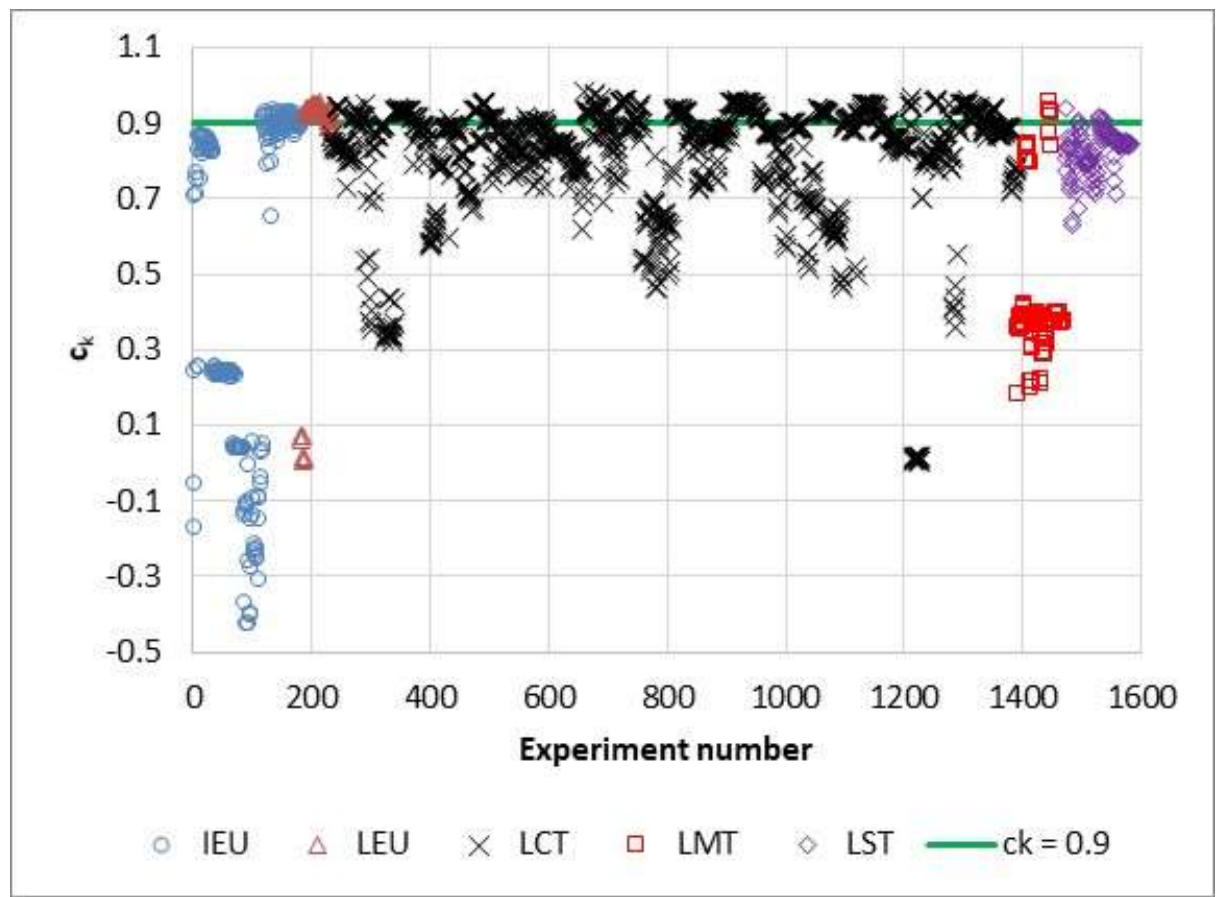

Figure 16. Traveller $c_{k}$ for the infinite array of packages with 10 wt. $\% \mathrm{UO}_{2}$ fuel rods.

Table 10. Traveller rod pipe model TSUNAMI-IP similarity summary.

\begin{tabular}{|c|c|c|}
\cline { 2 - 3 } \multicolumn{1}{c|}{} & \multicolumn{2}{c|}{ Experiments with $\boldsymbol{c}_{\boldsymbol{k}} \geq \mathbf{0 . 9}$} \\
\hline Category & $\mathbf{5}$ wt.\% UO2 rods & $\mathbf{1 0}$ wt.\% UO rods \\
\hline IEU $c_{k} \geq 0.9$ & 1 & 32 \\
\hline LEU $c_{k} \geq 0.9$ & 45 & 44 \\
\hline LCT $c_{k} \geq 0.9$ & 560 & 363 \\
\hline LMT $c_{k} \geq 0.9$ & 5 & 4 \\
\hline LST $c_{k} \geq 0.9$ & 1 & 6 \\
\hline Median $c_{k}$ & 0.873 & 0.860 \\
\hline
\end{tabular}




\section{3. $\mathrm{UO}_{2}$ POWDER AND PELLETS}

\subsection{CHT-OP-TU Package Description}

The CHT-OP-TU is a cube-shaped package designed to transport uranium oxide in powder or pellet form $[7,8] . \mathrm{UO}_{2}$ enrichment is currently limited to $\leq 5 \mathrm{wt} . \%{ }^{235} \mathrm{U}$. Overall dimensions are $45 \times 45 \times 62$ in. tall. Package construction is of 11 ga carbon or SS with foam and ceramic fiber insulation. $\mathrm{UO}_{2}$ is contained in four steel oxide vessels (OVs) placed within steel sleeves. Three different sizes of OVs may be used, which have nominal inside diameters of $8,7.5$, and 6 in. The OV minimum wall thickness is 0.22 in., and the maximum internal cavity height is $41 \mathrm{in}$. The vessels are arranged in a square lattice with a $22.5 \mathrm{in}$. pitch. Table 11 summarizes the modeled package dimensions, which are based on the HAC models in the SAR.

Table 11. CHT-OP-TU model data.

\begin{tabular}{lc}
\hline \multicolumn{1}{c}{ Parameter } & Value (cm) \\
\hline Outside width & 113.51 \\
\hline Outside length & 113.51 \\
\hline Outside height & 140.14 \\
\hline Outer steel wall thickness & 0.305 \\
\hline OV \#1 inner diameter & 20.824 \\
\hline OV \#2 inner diameter & 19.558 \\
\hline OV \#3 inner diameter & 15.748 \\
\hline OV maximum internal height & 105.18 \\
\hline OV minimum wall thickness & 0.5588 \\
\hline OV pitch & 56.992 \\
\hline Sleeve outer diameter & 27.305 \\
\hline Sleeve thickness & 0.366
\end{tabular}

Since package design drawings are redacted from the public version of the SAR, a SCALE 6.3.b11 KENO-VI HAC model was constructed by converting the SCALE 4.3 input provided in the SAR. HAC dimensions and conditions were chosen because they produced the highest SAR $k_{\text {eff. }}$ HAC model package dimensions are slightly different than the nominal design [8]. A cutaway representation of a $4 \times 4 \times 3$ array of 48 CHT-OP-TU packages is shown in Figure 17. The outermost region is water reflector. 


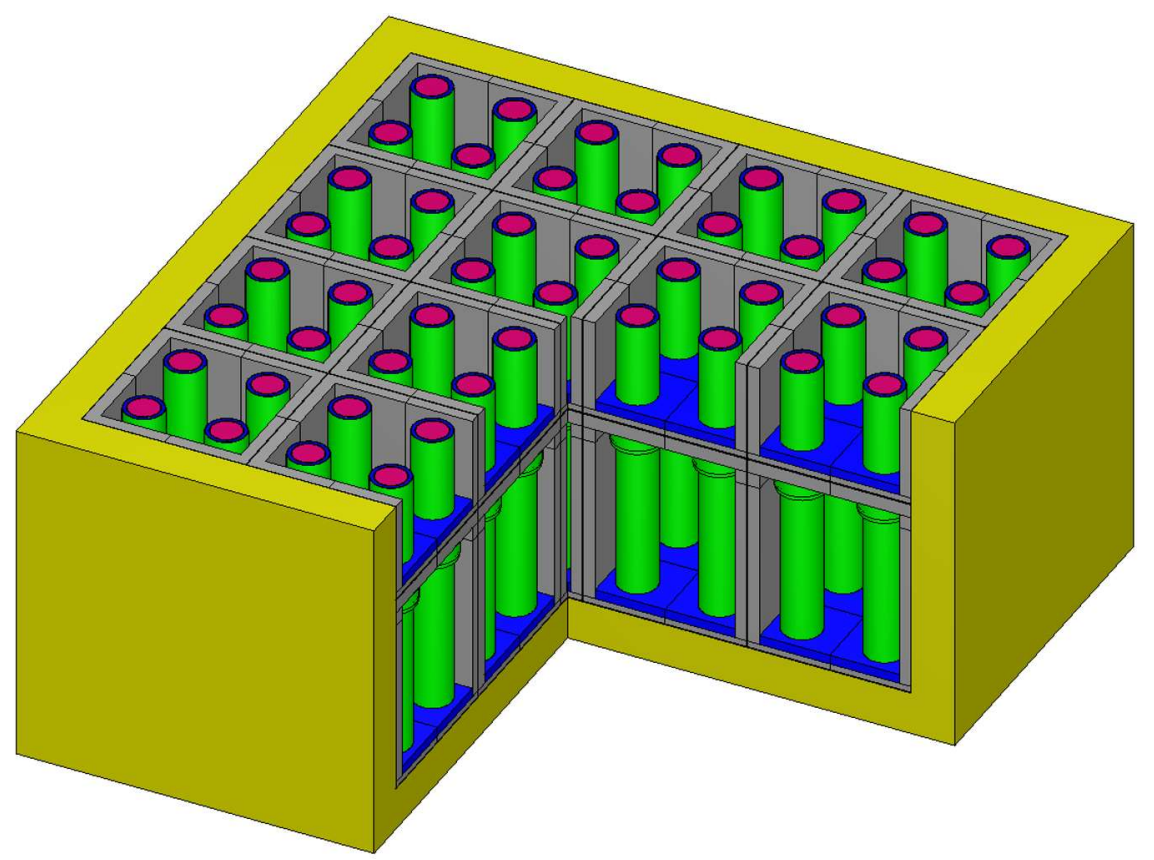

Figure 17. CHT-OP-TU model $\mathrm{UO}_{2}$ powder model $(4 \times 4 \times 3$ package array).

Two versions of the 48 package HAC array model were used to compare optimum moderation conditions and $k_{\text {eff }}$ with the CHT-OP-TU SAR results. Table 12 lists the CHT-OP-TU SAR HAC limiting conditions and $k_{\text {eff }}$ with $5 \mathrm{wt} . \%{ }^{235} \mathrm{U} \mathrm{UO}_{2}$ powder and $5 \mathrm{wt} . \%{ }^{235} \mathrm{U} \mathrm{UO}_{2}$ pellets. Polyethylene packaging increases $k_{\text {eff }}$ by $0.001-0.002$, but it is ignored in this study for simplicity. The SAR HAC array is $4 \times 4 \times 3+2$ (50 packages).

Table 12. CHT-OP-TU safety analysis bounding HAC results.

\begin{tabular}{cclc}
\hline $\begin{array}{c}\text { Number of } \\
\text { packages }\end{array}$ & Array size & \multicolumn{1}{c}{ Moderator } & $\boldsymbol{k}_{\text {eff }}$ \\
\hline 50 & $4 \times 4 \times 3+2$ & $\begin{array}{l}\text { Water reflector, homogenous water/ } \mathrm{UO}_{2} \text { mix in } 8 \text { in. OV, other } \\
\text { regions dry. } \mathrm{H} / \mathrm{X} \text { ratio } 150-200 .\end{array}$ & $0.9381 \pm 0.0007$ \\
\hline 50 & $4 \times 4 \times 3+2$ & $\begin{array}{l}\text { Water reflector, heterogenous water/UO } / \mathrm{UO}_{2} \text { mix in } 7.5 \mathrm{in.} \mathrm{OV}, \\
\text { other regions dry. OV water/fuel ratio } \sim 3.0 .\end{array}$ & $0.9255 \pm 0.0007$ \\
\hline
\end{tabular}

The KENO-VI $4 \times 4 \times 3$ array model was used to confirm HAC optimum moderation package configuration with a homogenous mixture of water and $\mathrm{UO}_{2}$. The array model has $30 \mathrm{~cm}$ of water reflection. KENO-VI and Sampler were used to calculate $k_{\text {eff }}$ with varying water volume fractions in the OV sleeve gap, in the internal package void space, and inside the $\mathrm{OV}\left(\mathrm{UO}_{2} \mathrm{vf}=1\right.$ - water VF). Fivehundred combinations of moderator VF were run with $k_{\text {eff }}$ statistical uncertainty (one sigma) $\leq 0.001$. Figure 18 shows the maximum $k_{\text {eff }}$ vs. water volume fraction for each of the three variables. The maximum $k_{\text {eff }}(0.941)$, which matches the SAR value within the uncertainty of the calculations, occurs with no water in the OV sleeve gap or in the package void spaces and $78 \%$ water VF in the 8 in. diameter OV $(\mathrm{H} / \mathrm{X}=196)$. 


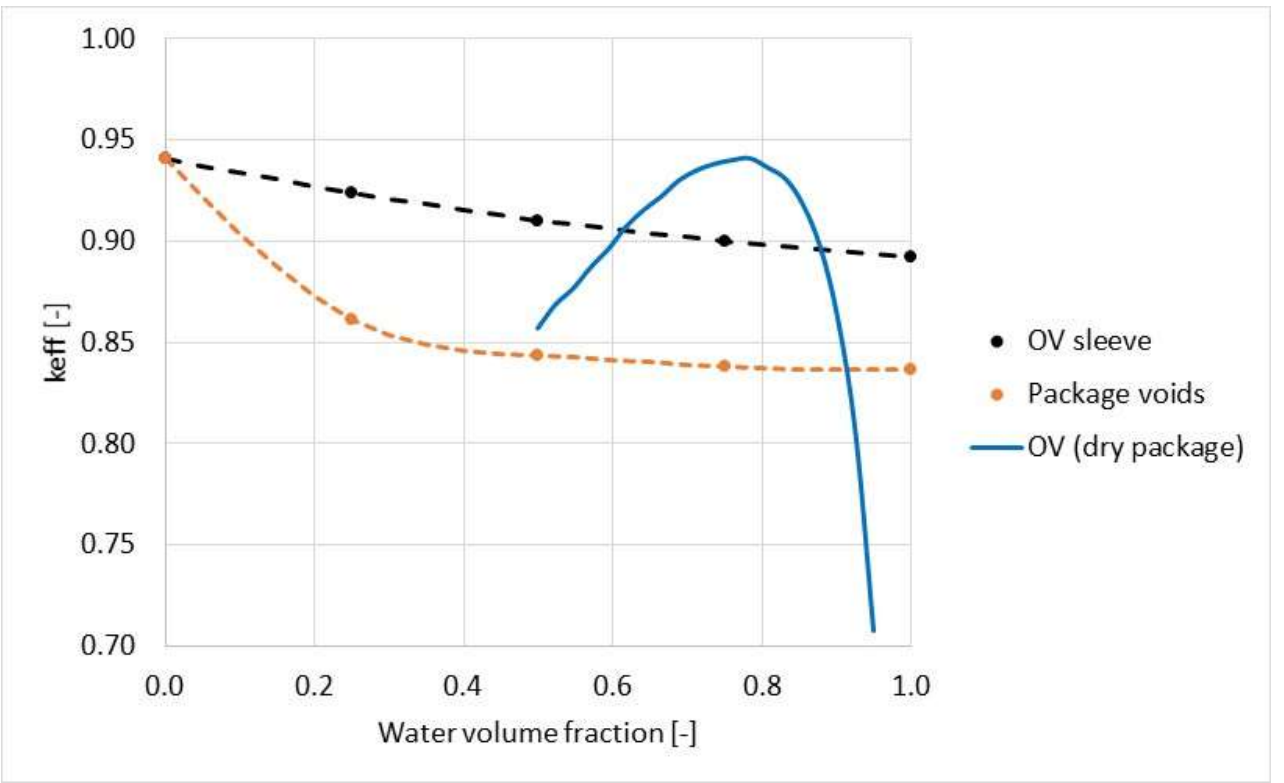

Figure 18. CHT-OP-TU UO $\mathrm{O}_{2}$ powder model optimum moderation results.

The KENO-VI $4 \times 4 \times 3$ package array pellet model was used to confirm HAC optimum package configuration with a heterogenous mixture of water and $\mathrm{UO}_{2}$ pellets. $\mathrm{UO}_{2}$ pellets were arranged in triangular pitch stacks. Partial pellets are included in the model where pellet stacks intersect the OV wall.

Sampler/KENO-VI was used to determine optimum pellet OD and pellet stack picth combinations with water filling the non-pellet space in the OV. Except for $30 \mathrm{~cm}$ of external water reflector, all other nonOV regions of the package were dry. A total of 256 combinations of pellet OD and pitch were run with $k_{\text {eff }}$ statistical uncertainty (one sigma) $\leq 0.0005$. Tighter convergence was used in this Sampler set to better resolve maximum reactivity conditions. Figure 19 shows $k_{\text {eff }}$ as a function of OD and pitch. There are several combinations of pellet OD and pitch at which statistically equivalent maximum $k_{\text {eff }}$ occurs. The largest best estimate value $(0.930)$ occurs with $0.6 \mathrm{~cm}$ pellet OD and $1.1 \mathrm{~cm}$ pitch, which corresponds to a water/fuel ratio of 2.7 . 


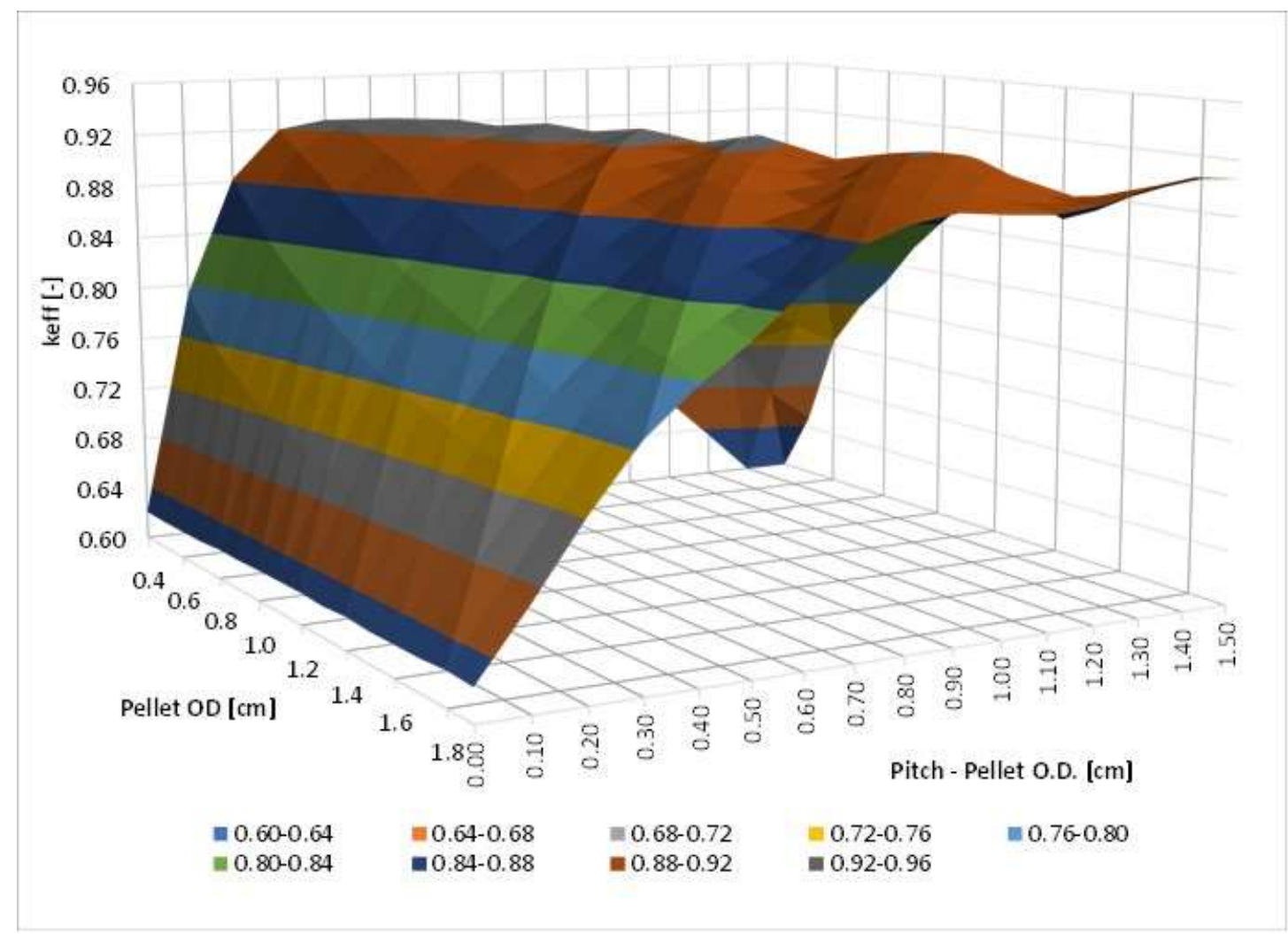

Figure 19. CHT-OP-TU UO $\mathrm{O}_{2}$ pellet model optimum moderation results.

The two $4 \times 4 \times 3$ HAC array KENO models produced bounding condition results very similar to the SAR results. There is little, if any, $k_{\text {eff }}$ margin available to accommodate increased enrichment without changing some aspect of the allowable configurations. Compensating for increased enrichment by reducing the HAC array size and reducing the OV diameter will be evaluated with the bounding models.

\subsection{CHT-OP-TU Powder Model Criticality Calculations}

Table 13 shows the variation of the $\mathrm{UO}_{2}$ powder model $k_{\text {eff }}$ with enrichment, HAC array size, and OV size. Combinations of these characteristics were varied to establish $k_{\text {eff }}$ close to 0.94 , which is the approximate upper bound from the SAR. Each case maintains the optimum moderator conditions determined in Section 3.1. Cube-shaped array configurations were selected to minimize neutron leakage. Although not comprehensive, the results in Table 13 suggest that by reducing the size of the HAC array and/or reducing the OV diameter, the CHT-OP-TU could be adapted to the transport of increased enrichment $\mathrm{UO}_{2}$. 
Table 13. CHT-OP-TU UO2 powder model criticality summary.

\begin{tabular}{|c|c|c|c|c|c|}
\hline $\begin{array}{c}\text { Enrichment } \\
\text { (wt.\%) }\end{array}$ & Array size & CSI & $\begin{array}{c}\text { OV diameter } \\
\text { (in.) }\end{array}$ & EALF (eV) \\
\hline 5 & $4 \times 4 \times 3$ & 2.1 & 8.0 & $0.9424 \pm 0.00049$ & 0.127 \\
\hline 6.25 & $4 \times 4 \times 3$ & 2.1 & 7.5 & $0.9420 \pm 0.00049$ & 0.150 \\
\hline 7.25 & $3 \times 3 \times 3$ & 3.8 & 7.5 & $0.9404 \pm 0.00049$ & 0.171 \\
\hline 8 & $3 \times 3 \times 2$ & 5.6 & 7.5 & $0.9416 \pm 0.00049$ & 0.189 \\
\hline 10 & $4 \times 4 \times 3$ & 2.1 & 6 & $0.8592 \pm 0.00049$ & 0.240 \\
\hline 18 & $4 \times 4 \times 3$ & 2.1 & 6 & $0.9331 \pm 0.00049$ & 0.507 \\
\hline $18^{*}$ & $4 \times 4 \times 3$ & 2.1 & 6 & $0.9390 \pm 0.00049$ & 0.314 \\
\hline
\end{tabular}

${ }^{*}$ After Sampler search for optimum moderation

The EALF of all cases indicates a thermal spectrum, which suggests that ample low-enriched, compound form, thermal energy spectrum (LCT) critical benchmark experiments are likely to be neutronically similar and useful for code validation. A review of 1,236 ICSBEP LCT experiment EALF values shows a range of 0.011 to $6.56 \mathrm{eV}$ with a median value of $0.24 \mathrm{eV}$ [14].

Given the large increase in enrichment considered, the optimum moderator conditions determined in Section 3.1 for $5 \mathrm{wt} . \% \mathrm{UO}_{2}$ might not be bounding for $18 \mathrm{wt} \% \mathrm{UO}_{2}$. A Sampler/KENO optimum moderation search was performed for the $18 \mathrm{wt} . \%$ case. The maximum $k_{\text {eff }}(0.9390)$ occurs with no water in the OV sleeve gap or in the package void spaces and $82.5 \%$ water VF in the 6 in. diameter OV.

The Table 13 results confirm that there are multiple combinations of package array and OV diameter that would support the transporation of $\mathrm{UO}_{2}$ powder enriched to $>5 \mathrm{wt} . \%{ }^{235} \mathrm{U}$.

\subsection{CHT-OP-TU Pellet Model Criticality Calculations}

Table 14 shows the variation of the $\mathrm{UO}_{2}$ pellet model $k_{\text {eff }}$ with enrichment, $\mathrm{HAC}$ array size, and OV size. Combinations of these characteristics were varied to establish a $k_{\text {eff }}$ close to 0.94 , which is the approximate upper bound from the SAR. Each case maintains the optimum moderator conditions determined in Section 3.1. Cube-shaped array configurations were selected to minimize neutron leakage. Although not comprehensive, the Table 13 results suggest that by reducing the size of the HAC array and/or reducing the OV diameter, the CHT-OP-TU could be adapted to transport increased enrichment $\mathrm{UO}_{2}$.

Table 14. CHT-OP-TU UO $\mathrm{O}_{2}$ pellet model criticality summary.

\begin{tabular}{|c|c|c|c|c|c|}
\hline $\begin{array}{c}\text { Enrichment } \\
\text { (wt.\%) }\end{array}$ & Array size & CSI & $\begin{array}{c}\text { OV diameter } \\
\text { (in.) }\end{array}$ & kALF (eV) \\
\hline 5 & $4 \times 4 \times 3$ & 2.1 & 7.5 & $0.9297 \pm 0.00049$ & 0.203 \\
\hline 5.5 & $4 \times 4 \times 3$ & 2.1 & 7.5 & $0.9397 \pm 0.00049$ & 0.212 \\
\hline 6.25 & $3 \times 3 \times 3$ & 3.8 & 7.5 & $0.9411 \pm 0.00049$ & 0.245 \\
\hline 6.9 & $3 \times 3 \times 2$ & 5.6 & 7.5 & $0.9408 \pm 0.00049$ & 0.271 \\
\hline 17 & $4 \times 4 \times 3$ & 2.1 & 6 & $0.9390 \pm 0.00049$ & 0.807 \\
\hline $17^{*}$ & $4 \times 4 \times 3$ & 2.1 & 6 & $0.9454 \pm 0.00049$ & 0.346 \\
16.5 & $4 \times 4 \times 3$ & 2.1 & 6 & $0.9423 \pm 0.00049$ & 0.334 \\
\hline
\end{tabular}

${ }^{*}$ After Sampler search for optimum moderation 
The EALF of all cases indicates a thermal spectrum, which suggests that ample low-enriched, compound form, thermal energy spectrum (LCT) critical benchmark experiments are likely to be neutronically similar and useful for code validation.

A Sampler/KENO optimum moderation search was performed for the $17 \mathrm{wt} . \%$ case. The maximum $k_{\text {eff }}$ (0.9454) occurs with no water in the OV sleeve gap or in the package void spaces, $0.6 \mathrm{~cm}$ pellet OD and $1.1 \mathrm{~cm}$ pitch (water/fuel ratio of 3.3).

The Table 14 results confirm that there are multiple combinations of package array and OV diameter that would support the transporation of $\mathrm{UO}_{2}$ pellets enriched to $>5 \mathrm{wt} . \%{ }^{235} \mathrm{U}$.

\subsection{CHT-OP-TU Powder Model TSUNAMI-3D Calculations}

TSUNAMI-3D S/U calculations were performed for two cases from Table 13. Table 15 shows key TSUNAMI input values, forward and adjoint $k_{\text {eff, }}$ the energy of the average lethargy of fission, and total cross section uncertainty.

Table 15. CHT-OP-TU UO O $_{2}$ powder model TSUNAMI-3D case data.

\begin{tabular}{|c|c|c|}
\hline Parameter & $\begin{array}{c}\mathbf{5} \text { wt.\%, 8 in. diameter } \\
\text { OV, 48 packages }\end{array}$ & $\begin{array}{c}\mathbf{8} \text { wt.\%, } \mathbf{7 . 5} \text { in. diameter OV, } \\
\mathbf{1 8} \text { packages }\end{array}$ \\
\hline Grid mesh & $50 \times 50 \times 30$ & $38 \times 38 \times 24$ \\
\hline Particles/gen. & $30,000 \mathrm{fwd} . / 90,000 \mathrm{adj}$. & $30,000 \mathrm{fwd} . / 90,000 \mathrm{adj}$. \\
\hline SDF file name & T3D_HAC_48_5wt_8in_rev3 & T3D_HAC_48_8wt_7-5in_rev3* \\
\hline Forward $k_{\text {eff }}$ & $0.94038 \pm 0.00049$ & $0.94136 \pm 0.00036$ \\
\hline Adjoint $k_{\text {eff }}$ & $0.9409 \pm 0.0014$ & $0.9427 \pm 0.0014$ \\
\hline EALF & $0.127 \mathrm{eV}$ & $0.189 \mathrm{eV}$ \\
\hline $\begin{array}{c}\text { Cross section } \\
\text { uncertainty }\end{array}$ & $0.658 \% \Delta \mathrm{k} / \mathrm{k}$ & $0.727 \% \Delta \mathrm{k} / \mathrm{k}$ \\
\hline
\end{tabular}

The adequacy of the TSUNAMI input is confirmed by comparing TSUNAMI-calculated nuclide sensitivity with KENO DP sensitivity. Figures 20 and 21 summarize the sensitivity results calculated by each method for the highest sensitivity nuclide/region combinations. There is good agreement between the DP and TSUNAMI sensitivities, which confirms that the TSUNAMI SDFs properly represent the models. The two models have the same sensitivity ranking and similar sensitivity values. 


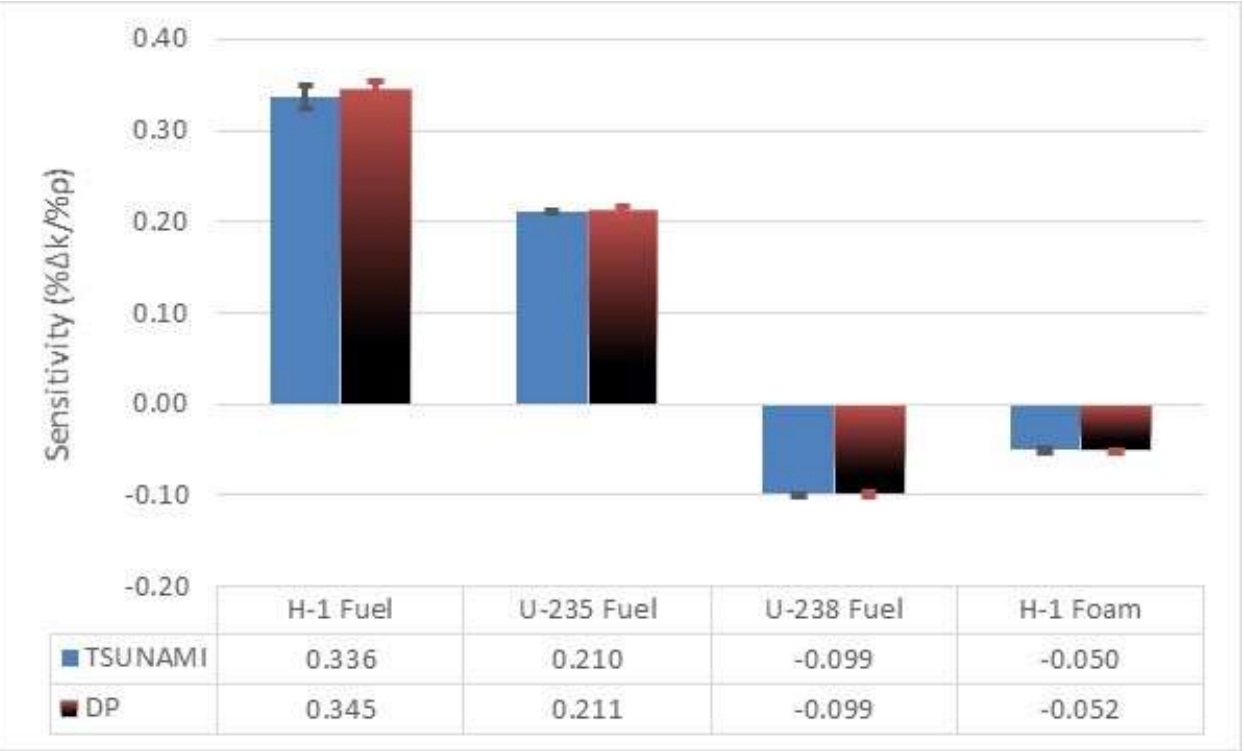

Figure 20. CHT-OP-TU UO2 powder model sensitivity (48 packages, 5 wt.\%, 8 in. OV).

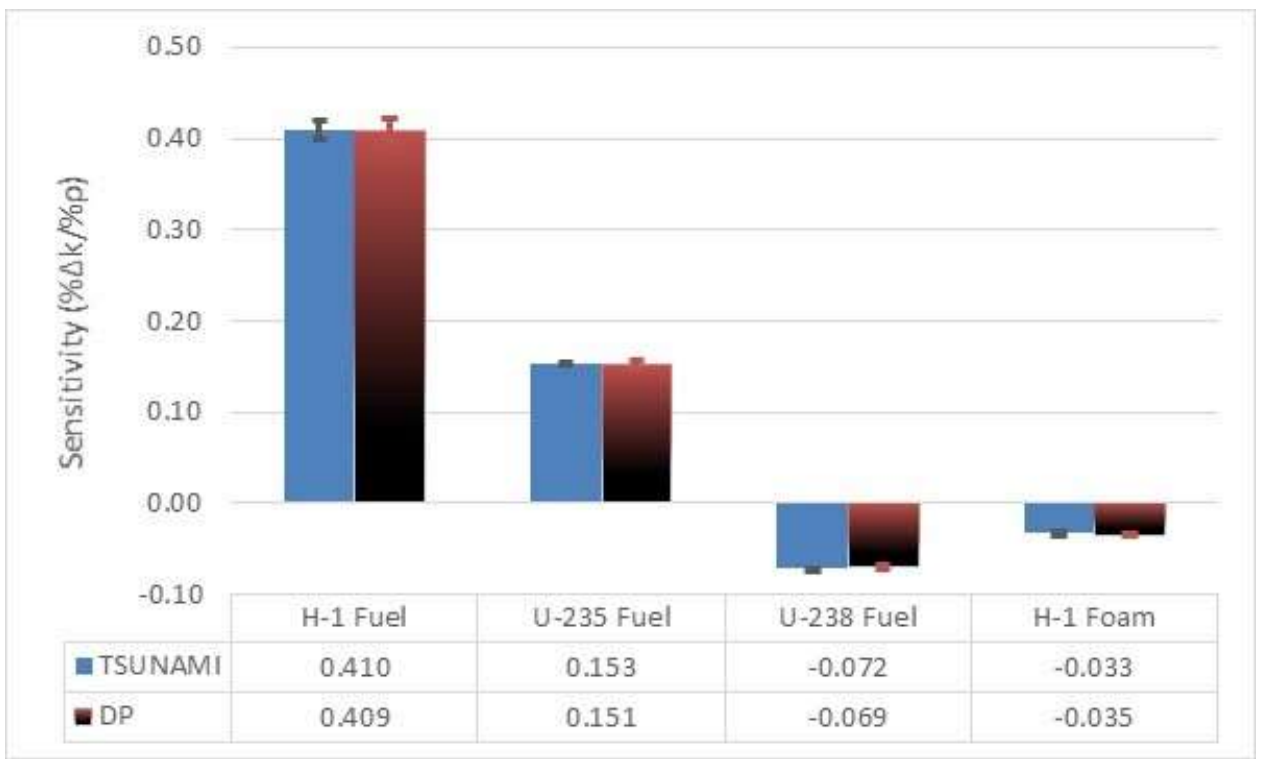

Figure 21. CHT-OP-TU UO2 powder model sensitivity (18 packages, 8 wt.\%, 7.5 in. OV).

Table 16 lists the sensitivity for each mixture in each model. Values greater than $0.01 \% \Delta \mathrm{k} / \% \Sigma$ are highlighted. The CHT-OP-TU models have few regions and materials. The $\mathrm{UO}_{2} /$ water mixture in the $\mathrm{OV}$, the OV and sleeve walls, and the low-density foam have the highest mixture sensitivities. 
Table 16. CHT-OP-TU UO ${ }_{2}$ powder model sensitivity by mixture.

\begin{tabular}{|c|c|c|c|c|c|}
\cline { 3 - 5 } \multicolumn{2}{c|}{} & \multicolumn{2}{c|}{$\mathbf{5}$ wt.\%, 8 in. diameter OV, } & \multicolumn{2}{c|}{$\mathbf{8}$ wt.\%, $\mathbf{7 . 5}$ in. diameter OV, } \\
\multicolumn{2}{c|}{} & \multicolumn{2}{|c|}{$\mathbf{4 8}$ packages } & \multicolumn{2}{c|}{ packages } \\
\hline Mixture & Description & Sensitivity & \% std. dev. & Sensitivity & \% std. dev. \\
\hline 1 & $\mathrm{UO}_{2}$ and water in OV & $\mathbf{0 . 4 8 2}$ & $\mathbf{1 . 4 \%}$ & $\mathbf{0 . 5 3 4}$ & $\mathbf{1 . 0 \%}$ \\
\hline 2 & Water outside OV & 0.000 & $3.4 \%$ & 0.000 & $3.9 \%$ \\
\hline 3 & Steel package wall & -0.007 & $6.9 \%$ & -0.005 & $7.8 \%$ \\
\hline 4 & OV and sleeve 304 SS & $\mathbf{- 0 . 0 3 4}$ & $\mathbf{2 . 8 \%}$ & $\mathbf{- 0 . 0 2 1}$ & $\mathbf{3 . 2 \%}$ \\
\hline 5 & Water reflector & 0.000 & N/A & 0.000 & N/A \\
\hline 6 & Low-density foam & $\mathbf{- 0 . 0 4 5}$ & $\mathbf{2 . 6 \%}$ & $\mathbf{- 0 . 0 2 6}$ & $\mathbf{4 . 0 \%}$ \\
\hline 7 & High-density foam & -0.008 & $6.5 \%$ & -0.004 & $10.2 \%$ \\
\hline 8 & Water in void spaces & 0.000 & $2.3 \%$ & 0.000 & $3.3 \%$ \\
\hline
\end{tabular}

\subsection{CHT-OP-TU UO 2 Powder Model TSUNAMI-IP Results}

TSUNAMI-IP was used to calculate correlation coefficients $\left(c_{k}\right)$ for the two SDF files in Table 15 and the library of 1,584 critical experiment SDF files. The $5 \mathrm{wt} . \%$ case serves as a reference point for comparison with higher enrichment results. Figures 22 and 23 show the similarity coefficients with different symbols representing different types of experiments. Table 17 shows the number of experiments in each category with $c_{k} \geq 0.9$ and the median $c_{k}$ for each CHT-OP-TU powder model. A full list of experiments with $c_{k}$ values is provided in Appendix A. The TSUNAMI results show that many experiments are likely to be applicable for code validation for the CHT-OP-TU powder models with $\mathrm{UO}_{2}$ fuel enrichment up to 8 wt.\% ${ }^{235} \mathrm{U}$. Enrichment higher than $8 \mathrm{wt} . \%$ is included in the pellet model evaluation.

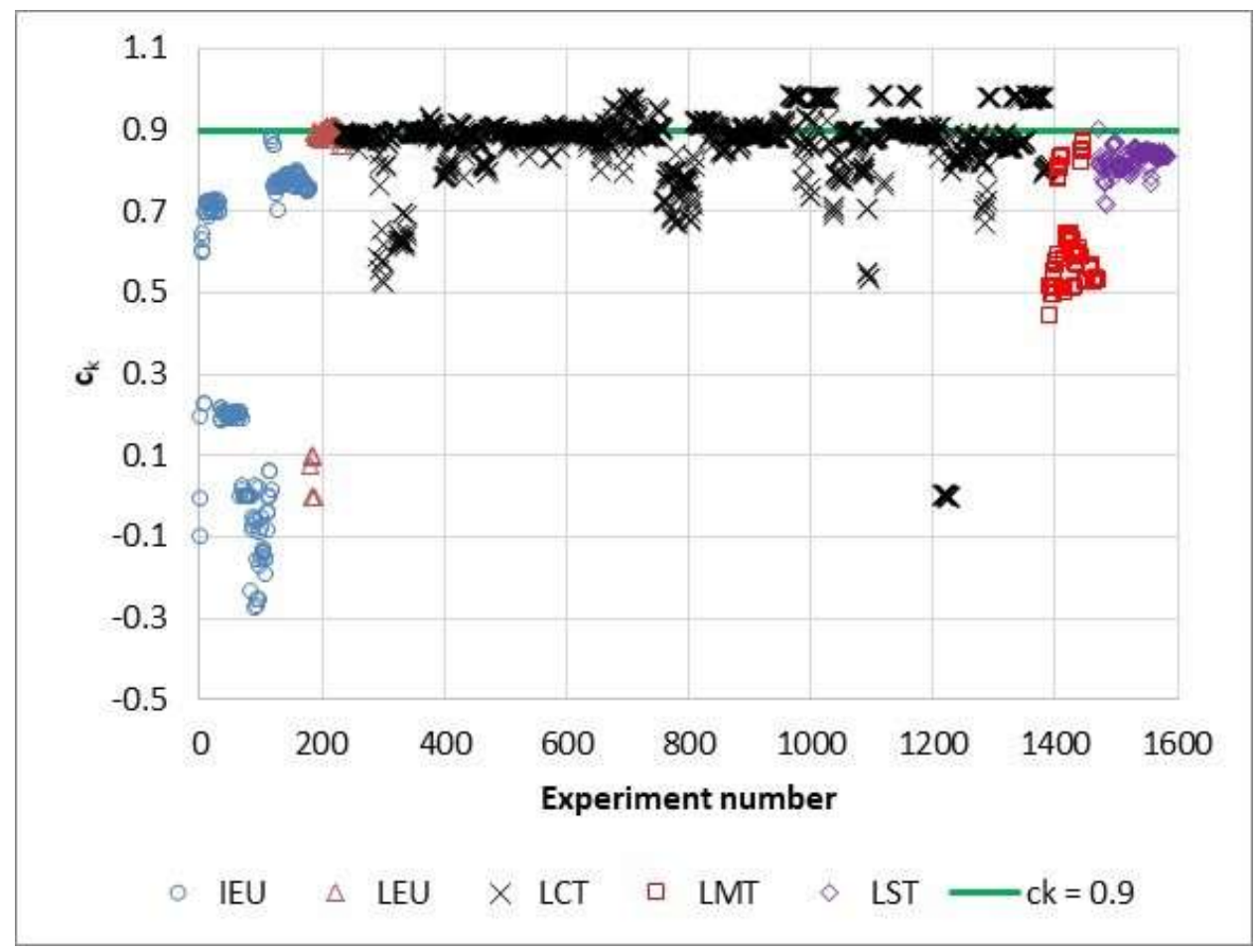

Figure 22. CHT-OP-TU $c_{k}$ for 5 wt. $\% \mathrm{UO}_{2}$ powder (48 packages, 8 in. diameter OV). 


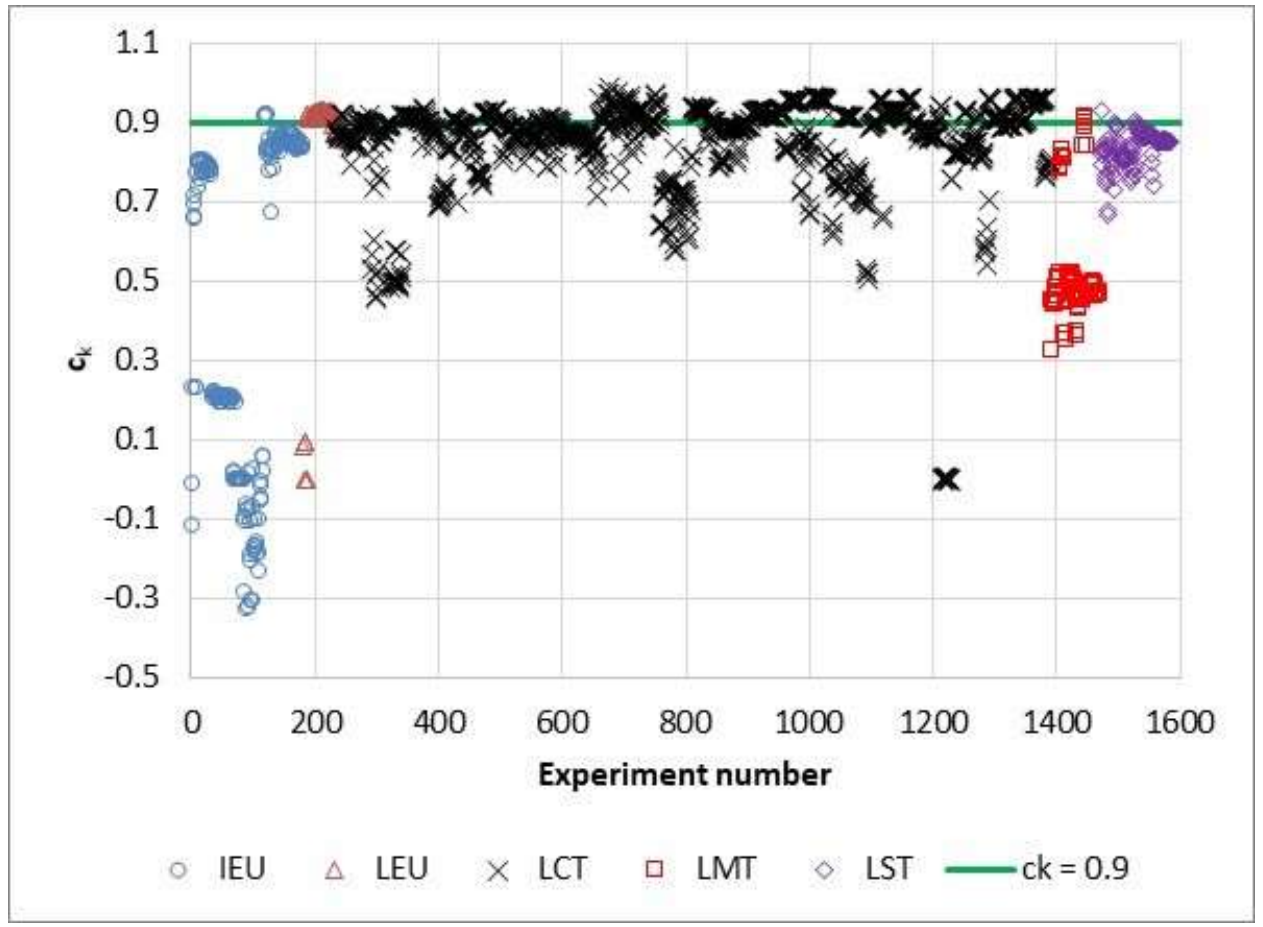

Figure 23. CHT-OP-TU $c_{k}$ for 8 wt.\% $\mathrm{UO}_{2}$ powder (18 packages, $7.5 \mathrm{in}$. diameter OV).

Table 17. CHT-OP-TU UO 2 powder model TSUNAMI-IP similarity summary.

\begin{tabular}{|c|c|c|}
\hline Category & 5 wt.\% & 8 wt.\% \\
\hline IEU $c_{k} \geq 0.9$ & 0 & 4 \\
LEU $c_{k} \geq 0.9$ & 18 & 44 \\
\hline LCT $c_{k} \geq 0.9$ & 301 & 476 \\
\hline LMT $c_{k} \geq 0.9$ & 0 & 3 \\
\hline LST $c_{k} \geq 0.9$ & 1 & 2 \\
\hline Median $c_{k}$ & 0.882 & 0.871 \\
\hline
\end{tabular}




\subsection{CHT-OP-TU Pellet Model TSUNAMI-3D Calculations}

TSUNAMI-3D S/U calculations were performed for two cases from Table 14. Table 18 shows key TSUNAMI input values, forward and adjoint $k_{\text {eff, }}$, the energy of the average lethargy of fission, and total cross section uncertainty.

Table 18. CHT-OP-TU UO 2 pellet model TSUNAMI-3D case data.

\begin{tabular}{|c|c|c|}
\hline Parameter & $\begin{array}{c}\text { 6.9 wt.\%, 7.5 in. diameter } \\
\text { OV } \mathbf{1 8} \text { packages }\end{array}$ & $\begin{array}{c}\mathbf{1 6 . 5} \text { wt. \%, 6 in. diameter OV } \\
\mathbf{4 8} \text { packages }\end{array}$ \\
\hline Grid mesh & $51 \times 51 \times 30$ & $50 \times 50 \times 30$ \\
\hline Particles/gen. & $100,000 \mathrm{fwd} . / 500,000 \mathrm{adj}$. & $30,000 \mathrm{fwd} . / 90,000 \mathrm{adj}$. \\
\hline SDF file name & $\begin{array}{c}\text { T3D_HAC_18_6-9wt_7- } \\
5 \mathrm{pel} \text { rev3a }\end{array}$ & T3D_HAC_48_16-5wt_6pel_rev3 \\
\hline Forward $k_{\text {eff }}$ & $0.94076 \pm 0.00039$ & $0.94247 \pm 0.00046$ \\
\hline Adjoint $k_{\text {eff }}$ & $0.9408 \pm 0.0011$ & $0.9427 \pm 0.0014$ \\
\hline EALF & $0.271 \mathrm{eV}$ & $0.334 \mathrm{eV}$ \\
\hline $\begin{array}{c}\text { Cross section } \\
\text { uncertainty }\end{array}$ & $0.720 \% \Delta \mathrm{k} / \mathrm{k}$ & $0.750 \% \Delta \mathrm{k} / \mathrm{k}$ \\
\hline
\end{tabular}

The adequacy of the TSUNAMI input is confirmed by comparing TSUNAMI-calculated nuclide sensitivity with KENO DP sensitivity. Figures 24 and 25 summarize the sensitivity results calculated by each method for the highest sensitivity nuclide/region combinations. There is good agreement between the DP and TSUNAMI sensitivities, which confirms that the TSUNAMI SDFs properly represent the models. The two models have the same sensitivity ranking and similar sensitivity values.

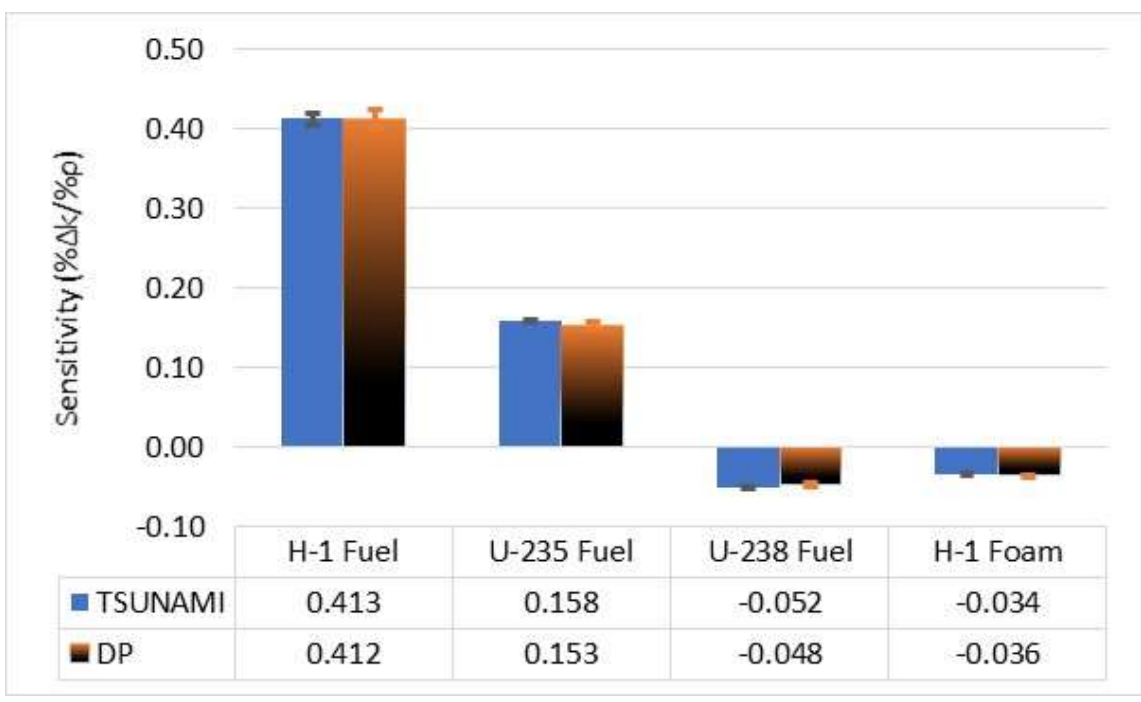

Figure 24. CHT-OP-TU UO 2 pellet model sensitivity (18 packages, 6.9 wt.\%, 7.5 in. OV). 


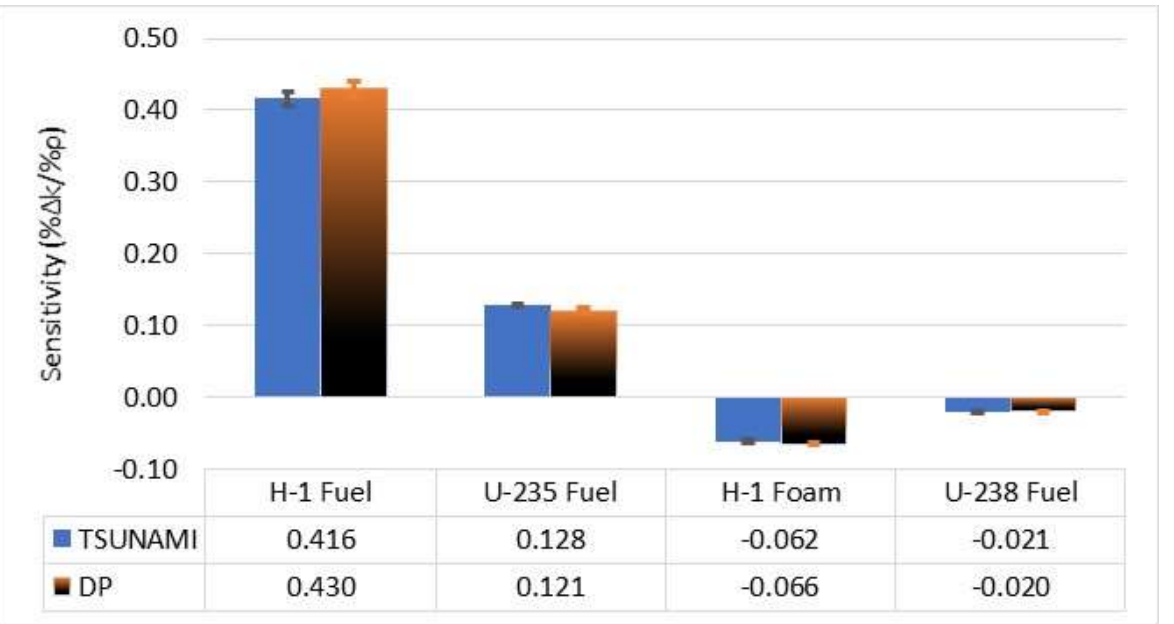

Figure 25. CHT-OP-TU UO 2 pellet model sensitivity (48 packages, 16.5 wt.\%, 6 in. OV).

Table 19 lists the sensitivity for each mixture in each model. Values greater than $0.01 \% \Delta \mathrm{k} / \% \Sigma$ are highlighted. The CHT-OP-TU models have few regions and materials. The $\mathrm{UO}_{2}$ and water mixtures in the $\mathrm{OV}, \mathrm{OV}$ and sleeve walls, and low-density foam have the highest mixture sensitivities.

Table 19. CHT-OP-TU UO 2 pellet model sensitivity by mixture.

\begin{tabular}{|c|c|c|c|c|c|}
\cline { 3 - 5 } \multicolumn{2}{c|}{} & \multicolumn{2}{c|}{$\begin{array}{c}\mathbf{6 . 9} \text { wt.\%, 7.5 in. diameter OV, } \\
\mathbf{1 8} \text { packages }\end{array}$} & \multicolumn{2}{c|}{$\begin{array}{c}\mathbf{1 6 . 5} \text { wt.\%, } \mathbf{6} \text { in. diameter OV, } \\
\mathbf{4 8} \text { packages }\end{array}$} \\
\hline Mixture & Description & Sensitivity & \% std. dev. & Sensitivity & $\%$ std. dev. \\
\hline 1 & UO $_{2}$ in OV & $\mathbf{0 . 1 2 1}$ & $\mathbf{0 . 5 \%}$ & $\mathbf{0 . 1 1 6}$ & $\mathbf{0 . 9 \%}$ \\
\hline 2 & Water outside OV & 0.000 & $2.2 \%$ & 0.000 & $2.1 \%$ \\
\hline 3 & Steel package wall & -0.005 & $4.8 \%$ & -0.009 & $5.3 \%$ \\
\hline 4 & OV and sleeve 304 SS & $\mathbf{- 0 . 0 2 2}$ & $\mathbf{1 . 7 \%}$ & $\mathbf{- 0 . 0 3 5}$ & $\mathbf{2 . 4 \%}$ \\
\hline 5 & Water reflector & 0.000 & N/A & 0.000 & N/A \\
\hline 6 & Low-density foam & $\mathbf{- 0 . 0 2 7}$ & $\mathbf{2 . 1 \%}$ & $\mathbf{- 0 . 0 5 4}$ & $\mathbf{2 . 1 \%}$ \\
\hline 7 & High-density foam & -0.005 & $5.6 \%$ & $\mathbf{- 0 . 0 1 1}$ & $\mathbf{4 . 8 \%}$ \\
\hline 8 & Water in void spaces & 0.000 & $1.7 \%$ & 0.000 & $1.8 \%$ \\
\hline 9 & Water in OV & $\mathbf{0 . 4 4 6}$ & $\mathbf{0 . 8 \%}$ & $\mathbf{0 . 4 4 8}$ & $\mathbf{1 . 1 \%}$ \\
\hline
\end{tabular}

\subsection{CHT-OP-TU UO O $_{2}$ Pellet Model TSUNAMI-IP Results}

TSUNAMI-IP was used to calculate correlation coefficients $\left(c_{k}\right)$ for the two SDF files in Table 18 and the library of 1,584 critical experiment SDF files. The $5 \mathrm{wt} . \%$ case serves as a reference point for comparison with higher enrichment results. Figures 26 and 27 show the similarity coefficients with different symbols representing different types of experiments. Table 20 shows the number of experiments in each category with $c_{k} \geq 0.9$ and the median $c_{k}$ for each of the CHT-OP-TU powder models. A full list of experiments with $c_{k}$ values is provided in Appendix A. The TSUNAMI results show that many experiments are likely to be applicable for code validation for the CHT-OP-TU pellet models with $\mathrm{UO}_{2}$ fuel enrichment up to $16.5 \mathrm{wt} . \%{ }^{235} \mathrm{U}$, although the $16.5 \mathrm{wt} . \%$ package median $c_{k}$ is substantially lower than for lower enrichment CHT-OP-TU models. 


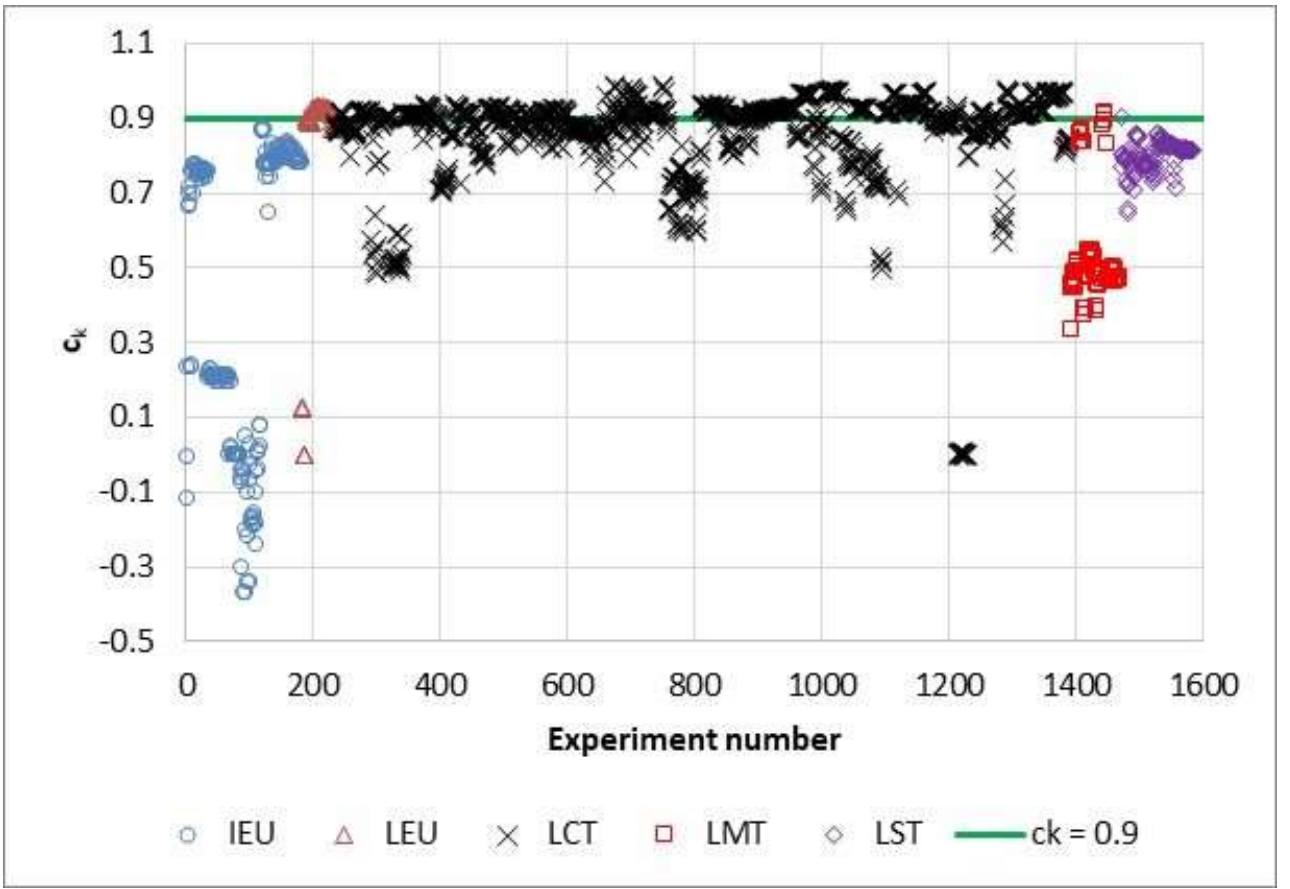

Figure 26. CHT-OP-TU $c_{k}$ for 6.9 wt.\% UO2 pellets (18 packages, 7.5 in. diameter OV).

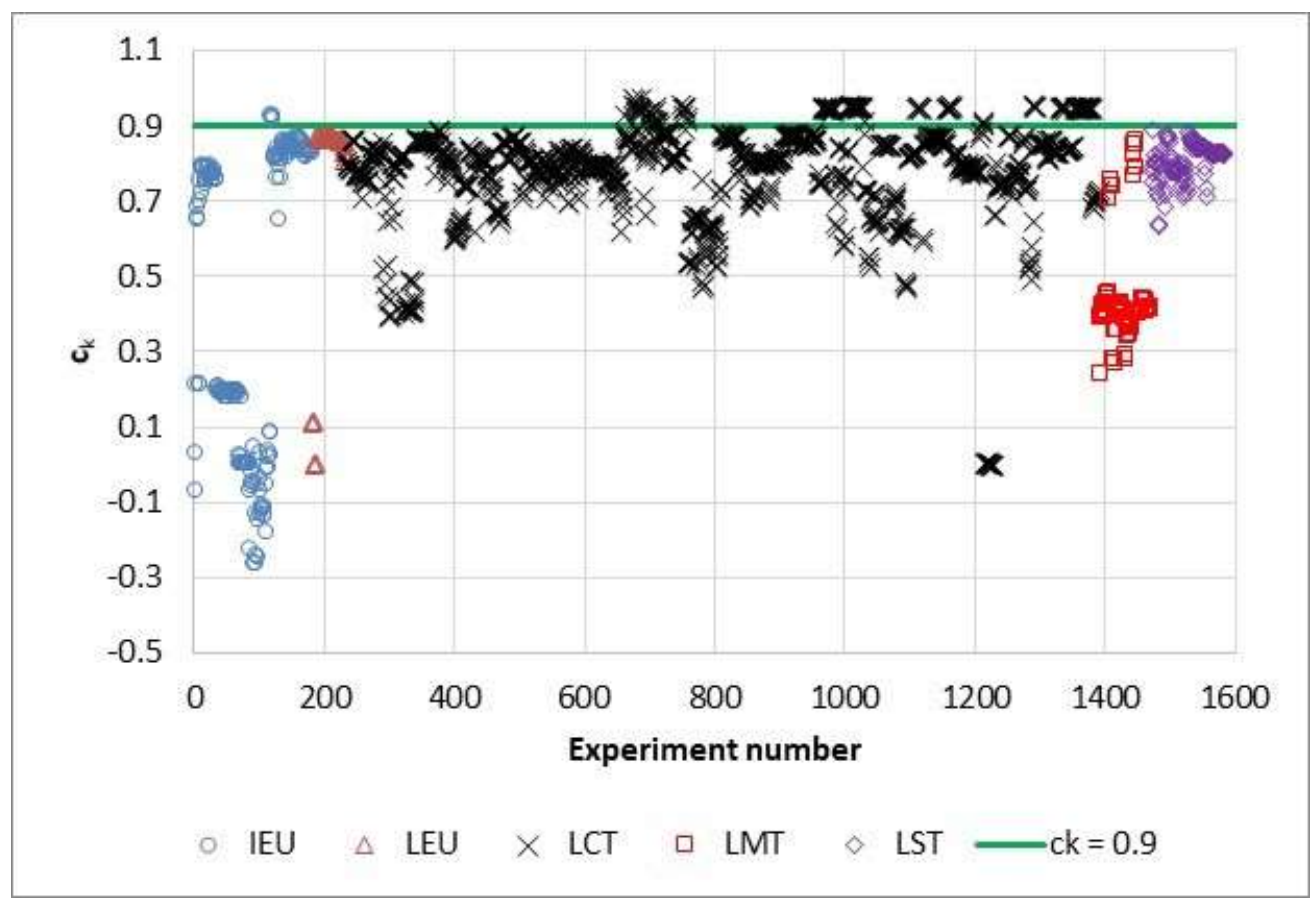

Figure 27. CHT-OP-TU $c_{k}$ for 16.5 wt.\% UO2 pellets (48 packages, 6 in. diameter OV). 
Table 20. CHT-OP-TU UO 2 pellet model TSUNAMI-IP similarity summary.

\begin{tabular}{|c|c|c|}
\hline Category & $\mathbf{6 . 9}$ wt.\% & $\mathbf{1 6 . 5}$ wt.\% \\
\hline IEU $c_{k} \geq 0.9$ & 0 & 4 \\
\hline LEU $c_{k} \geq 0.9$ & 37 & 0 \\
LCT $c_{k} \geq 0.9$ & 617 & 137 \\
\hline LMT $c_{k} \geq 0.9$ & 3 & 0 \\
\hline LST $c_{k} \geq 0.9$ & 1 & 0 \\
\hline Median $c_{k}$ & 0.881 & 0.807 \\
\hline
\end{tabular}




\section{U METAL AND ALLOYS}

\subsection{VERSA-PAC PACKAGE DESCRIPTION}

The Versa-Pac is a 55 or 110 gal drum-type package for shipping uranium oxides, uranium metal, uranyl nitrate crystals, uranium carbides, uranyl fluorides, uranyl carbonates, and uranium hexafluoride in $1 \mathrm{~S}$ or $2 \mathrm{~S}$ cylinders $[9,10]$. Figure 28 shows the major Versa-Pac components. A 5 in. carbon steel pipe container held in place by a "birdcage" device (not pictured) may optionally be used inside the containment body.

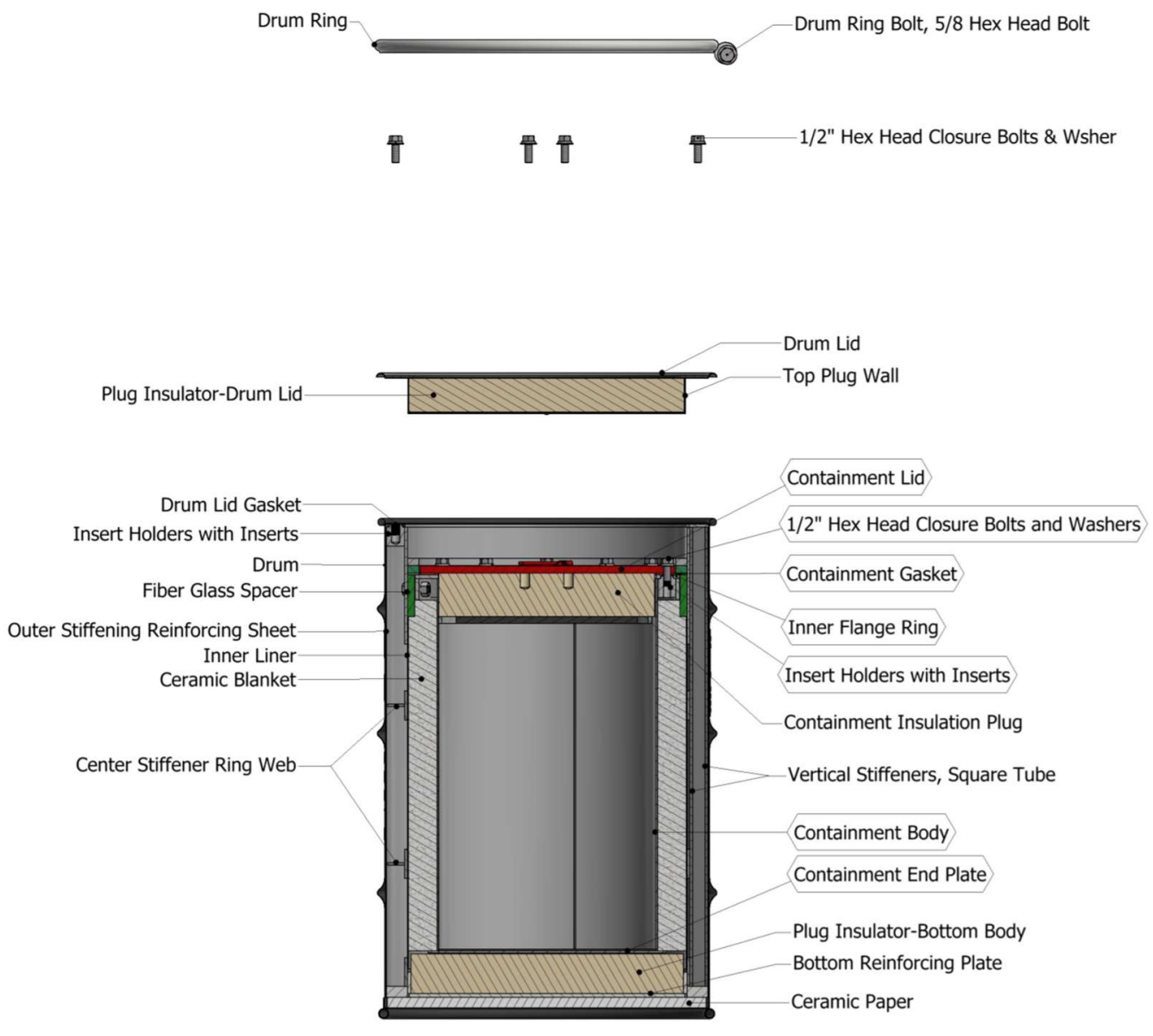

Figure 28. Versa-Pac component illustration [9].

The Versa-Pac package is currently licensed to carry $U$ materials enriched up to $100 \%{ }^{235} \mathrm{U}$ with a ${ }^{235} \mathrm{U}$ content maximum allowed mass determined by enrichment bin, as shown in Table 21. Because the VersaPac is already licensed to transport HALEU and has mass limits that are a function of enrichment, no additional evaluation is required. 
Table 21. Versa-Pac maximum ${ }^{235} \mathrm{U}$ per package (ground/vessel transportation) [15].

\begin{tabular}{ccc}
\hline${ }^{235} \mathbf{U}$ wt.\% & Maximum grams ${ }^{\mathbf{2 3 5}} \mathbf{U}$ & Packaging type \\
\hline$\leq 100$ & 350 & 55 or 110 gal drum \\
\hline 20 & 410 & 55 or 110 gal drum \\
$\leq 10$ & 470 & 55 or 110 gal drum \\
\hline 5 & 580 & 55 or 110 gal drum \\
$\leq 20$ & 605 & $\begin{array}{c}55 \text { or } 110 \text { gal drum, limited } \\
\text { hydrogenous packing, CSI }=0.7\end{array}$ \\
& & $\begin{array}{c}55 \text { or } 110 \text { gal drum, limited } \\
\text { hydrogenous packing, CSI }=1.0\end{array}$ \\
\hline 20 & 635 & 55 gal drum, 5 in. pipe \\
\hline 100 & 695 & 55 gal drum, 5 in. pipe \\
\hline 20 & 1,215 & 55 gal drum, 5 in. pipe \\
\hline 10 & 1,605 & 55 gal drum, 5 in. pipe \\
\hline$\leq 5$ & 1,065 &
\end{tabular}




\section{BWR FUEL RODS AND FUEL ASSEMBLIES}

\subsection{Framatome TN-B1 Package Description}

The TN-B1 is currently licensed to transport two commercial BWR fuel assemblies with lattice average ${ }^{235} \mathrm{U}$ enrichment $\leq 5.0 \mathrm{wt} . \%$ or various types of unirradiated fuel rods with ${ }^{235} \mathrm{U}$ enrichment $\leq 5.0 \mathrm{wt} . \%$ in a 5 in. SS rod pipe $[11,12]$. Multiple fuel assembly design requirements (i.e., Gd rods) and restrictions (i.e., fuel rod OD) are included in the licensing basis that are primarily a function of lattice size.

The TN-B1 package is a rectangular box 29.21 in. tall, $28.35 \mathrm{in}$. wide, and $199.53 \mathrm{in}$. long that comprises one inner and one outer SS container. The inner container has a double-wall SS sheet structure with an alumina silicate thermal insulator and foam polyethylene cushioning material. The outer container comprises an angular framework covered with SS plates. A representation of the TN-B1 package from the safety analysis is shown in Figure 29.

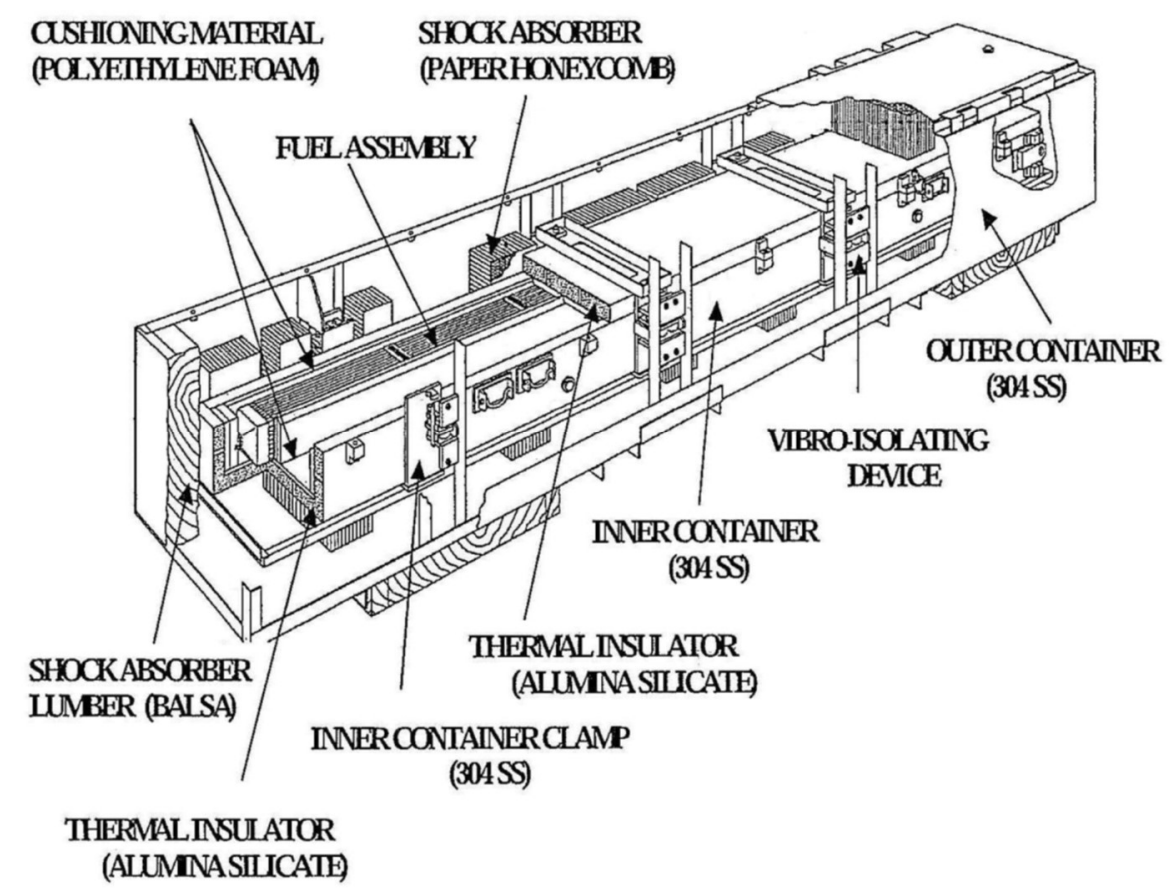

Figure 29. TN-B1 package assembly [11].

The most limiting configuration of the TN-B1 package identified in the criticality safety analysis is the HAC $10 \times 1 \times 10$ array of packages; no space between packages; full flooding in the inner container, including the fuel pellet-clad gap; no water in the outer container or between packages; and $30 \mathrm{~cm}$ of water reflector. HAC model dimensions from the criticality safety analysis are shown in Figure 30. Package reactivity is partly controlled by requiring a minimum number of $\mathrm{UO}_{2}$ rods that contain $2.0 \mathrm{wt} . \%$ $\mathrm{Gd}_{2} \mathrm{O}_{3}$ rods (Gd rods) in the fuel assembly lattice. The $5.0 \mathrm{wt} . \%{ }^{235} \mathrm{U} 11 \times 11$ assembly design requires $13 \mathrm{Gd}$ rods and is the reference design used in this study. 


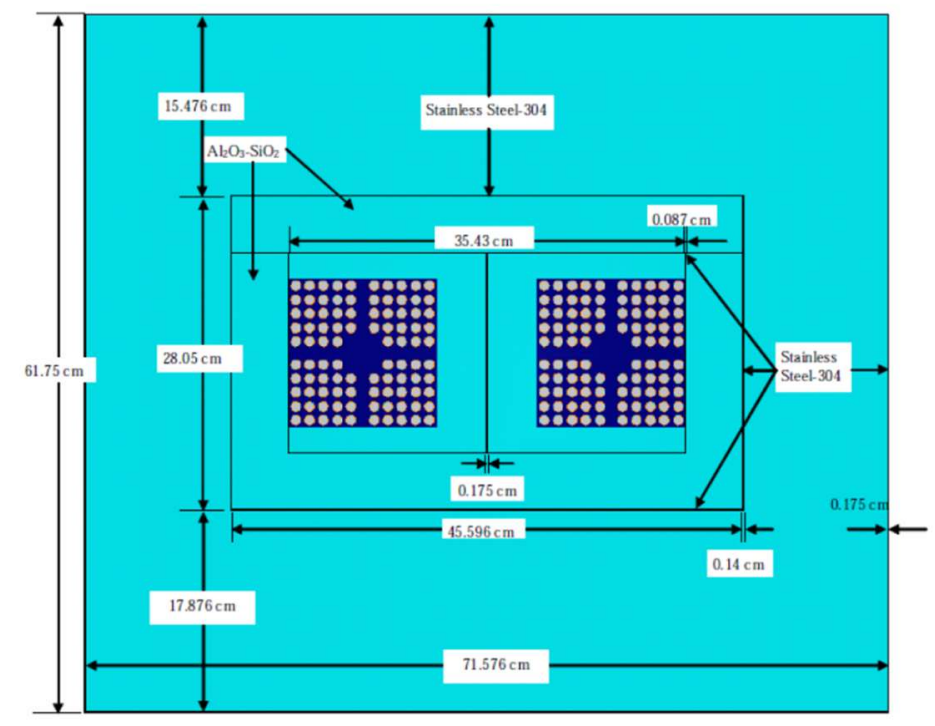

Figure 30. TN-B1 HAC package dimensions [11].

\subsection{TN-B1 SCALE KENO-VI Models}

A SCALE 6.3.b11 KENO-VI TN-B1 HAC model was constructed by converting a KENO-V.a model provided in the SAR. This base case model consists of a $10 \times 1 \times 10$ array of packages with each package containing two $11 \times 115$ wt.\% fuel assemblies. Each assembly has $13 \mathrm{Gd}$ rods and three axial regions, which differ in the number and configuration of empty lattice locations. Fuel enrichment is $5 \mathrm{wt} . \%$ in all fuel rods and axial regions. The base case is used as a representative of TN-B1 characteristics with other fuel designs. The $k_{\text {eff }}$ of this HAC case is used as a target for the increased enrichment study. NCT conditions are generally less limiting than HAC conditions and are not modeled for this evaluation.

The effect of increased fuel enrichment fuel in the TN-B1 can be offset by requiring more Gd rods or by reducing the size of the package array. Both approaches are considered in the evaluation. Two cutaway views of a $6 \times 1 \times 6$ package array (top section and front half removed) surrounded by $30 \mathrm{~cm}$ of water are shown in Figure 31. 

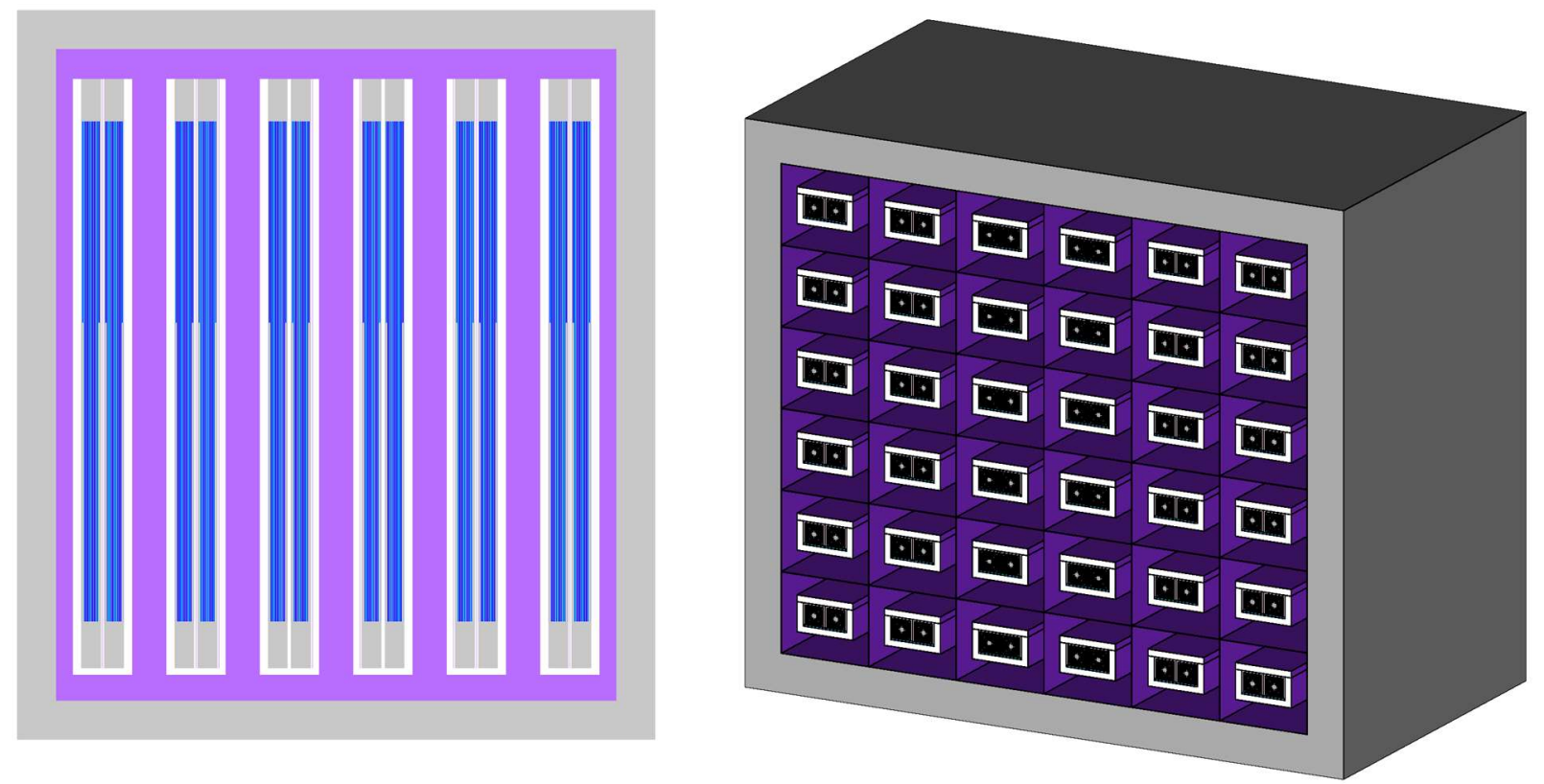

Figure 31. TN-B1 $6 \times 1 \times 6$ package array top and front cutaway views.

\subsection{TN-B1 Criticality Calculations}

Only one fuel design is used because the objective of this calculation is to assess the effect of increased enrichment. This objective can be met with a representative fuel assembly. Bounding moderator conditions are the same as those determined in the TN-B1 SAR and are not separately verified herein.

Table 22 shows the results of TN-B1 criticality calculations. In the first series of cases, HAC array size is varied from $10 \times 1 \times 10$ to $2 \times 1 \times 2$ incrementally with $\mathrm{UO}_{2}$ enrichment fixed at $5 \mathrm{wt} . \%$. In the second set, enrichment is increased until $k_{\text {eff }}$ is approximately the same as the base case. The last case represents a $10 \times 1 \times 10 \mathrm{HAC}$ array of packages that contain $8 \mathrm{wt} . \%$ fuel assemblies wit $24 \mathrm{Gd}$ rods.

Table 22. TN-B1 HAC array criticality calculations.

\begin{tabular}{|c|c|c|c|c|c|c|}
\hline $\begin{array}{c}\text { Enrichment } \\
\left(\mathbf{w t . \%}{ }^{235} \mathbf{U}\right)\end{array}$ & Gd $^{*}$ rods & Array size & CSI & $\begin{array}{c}\text { Fuel } \\
\text { assemblies }\end{array}$ & $\boldsymbol{k}_{\text {eff }}$ & $\begin{array}{c}\text { EALF } \\
(\mathbf{e V})\end{array}$ \\
\hline 5 & 13 & $2 \times 1 \times 2$ & 25 & 8 & $0.7108 \pm 0.00031$ & 0.293 \\
\hline 5 & 13 & $3 \times 1 \times 3$ & 12.5 & 18 & $0.7724 \pm 0.00036$ & 0.275 \\
\hline 5 & 13 & $4 \times 1 \times 4$ & 6.3 & 32 & $0.8184 \pm 0.00033$ & 0.267 \\
\hline 5 & 13 & $5 \times 1 \times 5$ & 4.2 & 50 & $0.8528 \pm 0.00031$ & 0.260 \\
\hline 5 & 13 & $6 \times 1 \times 6$ & 2.8 & 72 & $0.8783 \pm 0.00035$ & 0.256 \\
\hline 5 & 13 & $7 \times 1 \times 7$ & 2.1 & 98 & $0.8991 \pm 0.00030$ & 0.253 \\
\hline 5 & 13 & $8 \times 1 \times 8$ & 1.6 & 128 & $0.9148 \pm 0.00030$ & 0.251 \\
\hline 5 & 13 & $9 \times 1 \times 9$ & 1.3 & 162 & $0.9271 \pm 0.00031$ & 0.249 \\
\hline 5 & 13 & $10 \times 1 \times 10$ & 1 & 200 & $0.9374 \pm 0.00036$ & 0.249 \\
\hline 5.55 & 13 & $8 \times 1 \times 8$ & 1.6 & 128 & $0.9371 \pm 0.00031$ & 0.269 \\
\hline 6 & 13 & $7 \times 1 \times 7$ & 2.1 & 98 & $0.9371 \pm 0.00031$ & 0.288 \\
\hline
\end{tabular}




\begin{tabular}{|c|c|c|c|c|c|c|}
6.7 & 13 & $6 \times 1 \times 6$ & 2.8 & 72 & $0.9377 \pm 0.00033$ & 0.316 \\
\hline 7.8 & 13 & $5 \times 1 \times 5$ & 4.2 & 50 & $0.9371 \pm 0.00036$ & 0.366 \\
\hline 9.8 & 13 & $4 \times 1 \times 4$ & 6.3 & 32 & $0.9374 \pm 0.00041$ & 0.465 \\
\hline 8 & 24 & $10 \times 1 \times 10$ & 1 & 200 & $0.9380 \pm 0.00034$ & 0.454 \\
\hline
\end{tabular}

${ }^{*} 2.0$ wt. $\% \mathrm{Gd}_{2} \mathrm{O}_{3}$

Figure 32 illustrates the trade-off of increased enrichment and reduced array size. Figure 33 compares the $10 \times 1 \times 10$ array $24 \mathrm{Gd}$ rod requirement to extrapolated Gd rod vs. $11 \times 11$ fuel enrichment data from the TN-B1 CoC [17]. A log-linear fit line extrapolation provides a good estimate of the number of Gd rods required.

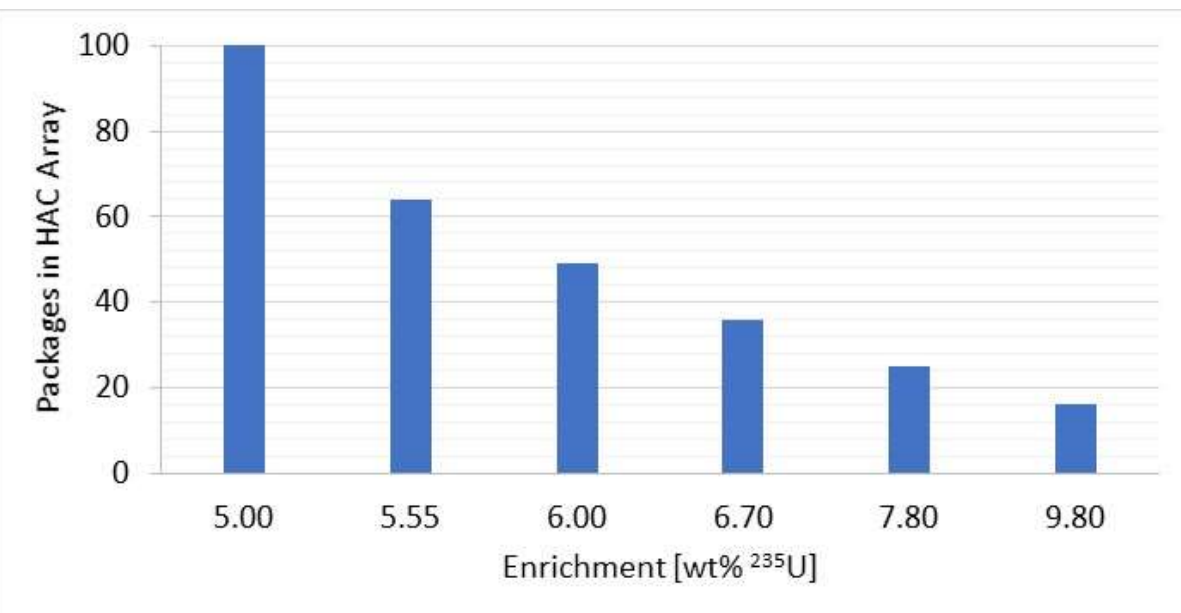

Figure 32. TN-B1 package array size vs. fuel assembly enrichment.

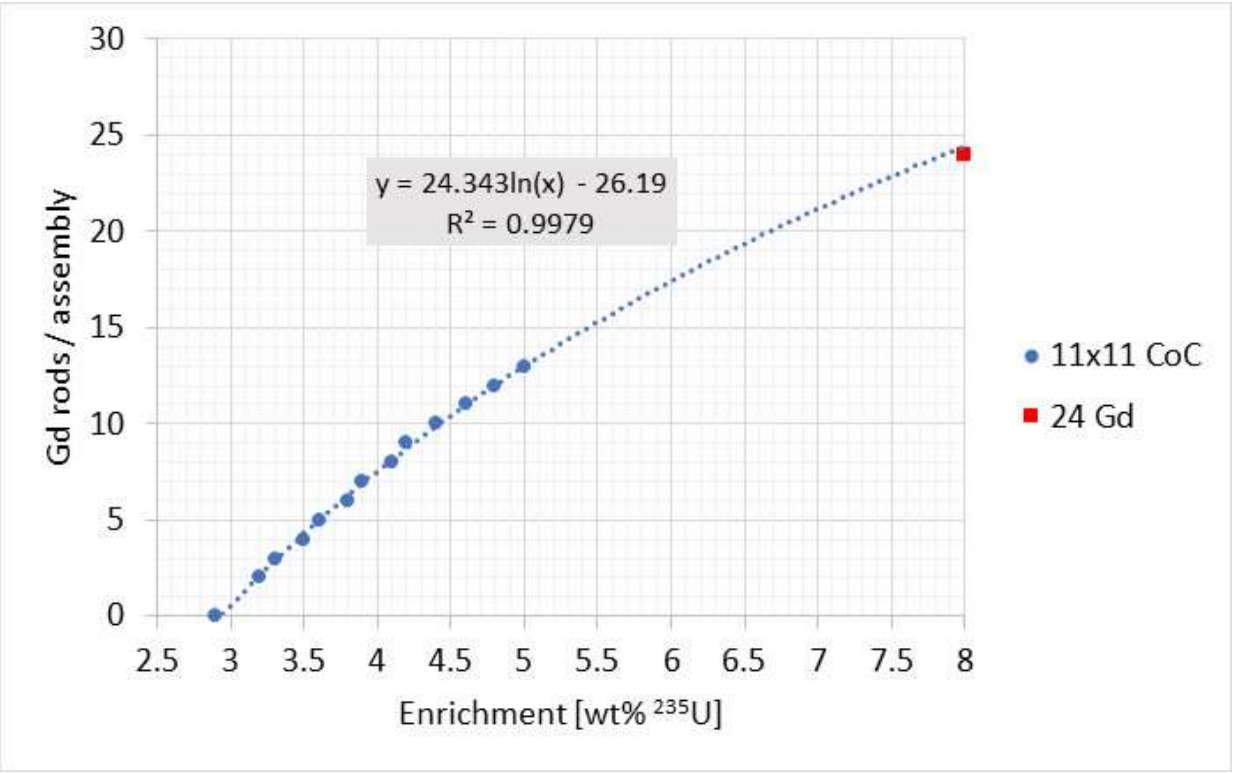

Figure 33. TN-B1 $10 \times 1 \times 10$ package Gd rods required vs. fuel assembly enrichment. 
These results show that the TN-B1 could be used for the transportation of increased enrichment fuel by reducing the size of transportation arrays, increasing the number of Gd rods, or a combination of the two. From this set of cases, three were selected for TSUNAMI similarity calculations that cover a range of enrichment with reasonable transportation array size:

1) $5.0 \mathrm{wt} . \%{ }^{235} \mathrm{U}, 10 \times 1 \times 10$ array, $13 \mathrm{Gd}$ rods/fuel assembly;

2) $6.7 \mathrm{wt} . \%{ }^{235} \mathrm{U}, 6 \times 1 \times 6$ array, $13 \mathrm{Gd}$ rods/fuel assembly; and

3) $8.0 \mathrm{wt} . \%{ }^{235} \mathrm{U}, 10 \times 1 \times 10$ array, $24 \mathrm{Gd}$ rods/fuel assembly.

\subsection{TN-B1 TSUNAMI-3D Calculations}

TSUNAMI-3D S/U calculations were performed for three cases from Table 22. Table 23 shows key TSUNAMI input values, forward and adjoint $k_{\text {eff, }}$, the energy of the average lethargy of fission, and total cross section uncertainty.

Table 23. TN-B1 HAC model TSUNAMI-3D case data.

\begin{tabular}{|c|c|c|c|}
\hline Parameter & $\begin{array}{c}\mathbf{5 . 0} \text { wt.\%, 10 } \times \mathbf{1} \times \mathbf{1 0} \\
\text { package array, 13 Gd rods }\end{array}$ & $\begin{array}{c}\mathbf{6 . 7} \mathbf{w t . \%}, \mathbf{6} \times \mathbf{1} \times \mathbf{6} \\
\text { package array, 13 Gd rods }\end{array}$ & $\begin{array}{c}\mathbf{8 . 0} \text { wt.\%, 10 } \times \mathbf{1} \times \mathbf{1 0} \\
\text { package array, 24 Gd rods }\end{array}$ \\
\hline Grid mesh & $52 \times 52 \times 40$ & $38 \times 38 \times 40$ & $52 \times 52 \times 40$ \\
\hline Particles/gen. & $100,000 \mathrm{fwd} . / 300,000 \mathrm{adj}$. & $50,000 \mathrm{fwd} . / 150,000 \mathrm{adj}$. & $100,000 \mathrm{fwd} . / 300,000$ adj. \\
\hline SDF file name & T3D_HAC_5wt_13Gd_10x10a & T3D_HAC_6-7wt_13Gd_6x6 & T3D_HAC_8wt_24Gd_10x10a \\
\hline Forward $k_{\text {eff }}$ & $0.93688 \pm 0.00037$ & $0.93679 \pm 0.00031$ & $0.93763 \pm 0.00027$ \\
\hline Adjoint $k_{\text {eff }}$ & $0.9364 \pm 0.0011$ & $0.9373 \pm 0.0014$ & $0.9370 \pm 0.0011$ \\
\hline EALF & $0.248 \mathrm{eV}$ & $0.317 \mathrm{eV}$ & $0.454 \mathrm{eV}$ \\
\hline $\begin{array}{c}\text { Cross section } \\
\text { uncertainty }\end{array}$ & $0.536 \% \Delta \mathrm{k} / \mathrm{k}$ & $0.568 \% \Delta \mathrm{k} / \mathrm{k}$ & $0.522 \% \Delta \mathrm{k} / \mathrm{k}$ \\
\hline
\end{tabular}

The adequacy of the TSUNAMI input was confirmed by comparing TSUNAMI-calculated nuclide sensitivity with KENO DP sensitivity. Figures 34, 35, and 36 summarize the sensitivity results calculated by each method for the highest sensitivity nuclide/region combinations. There is good agreement between the DP and TSUNAMI sensitivities, which confirms that the TSUNAMI SDFs properly represent the models. The two models have the same sensitivity ranking and similar sensitivity values.

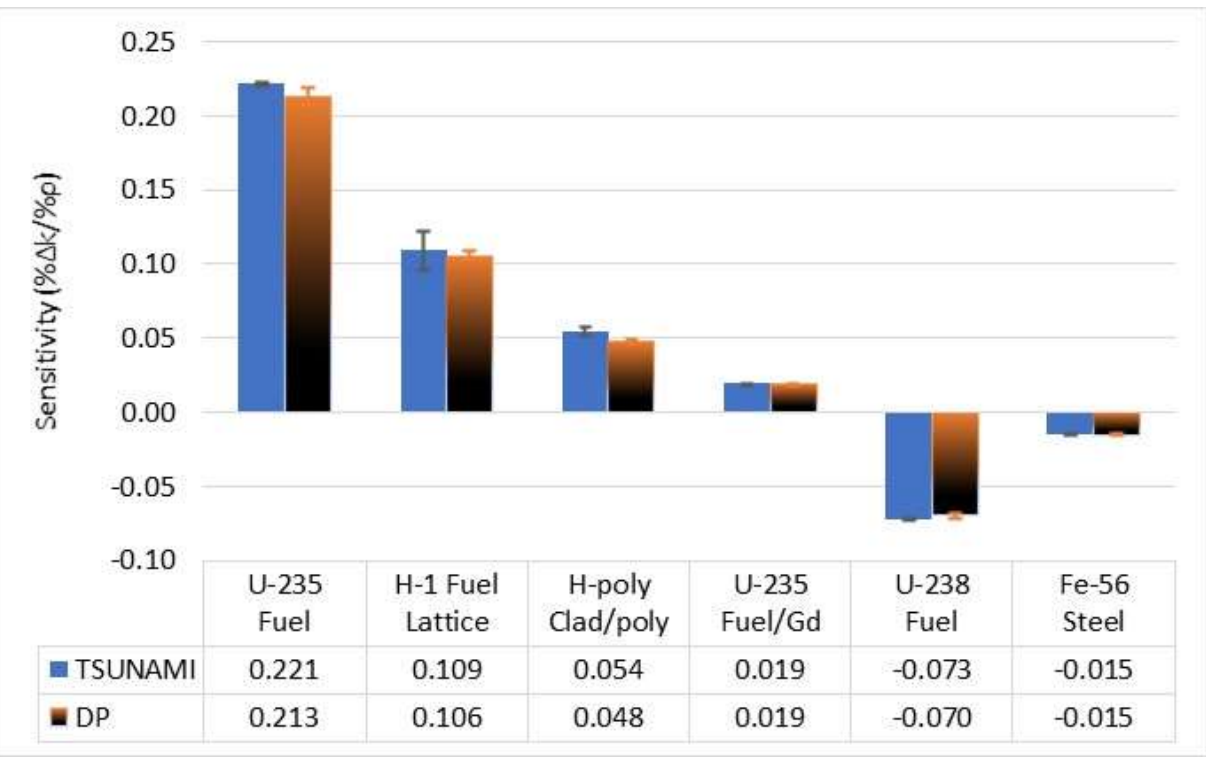


Figure 34. TN-B1 model sensitivity (100 packages, 5 wt.\%, 13 Gd rods/fuel assembly).

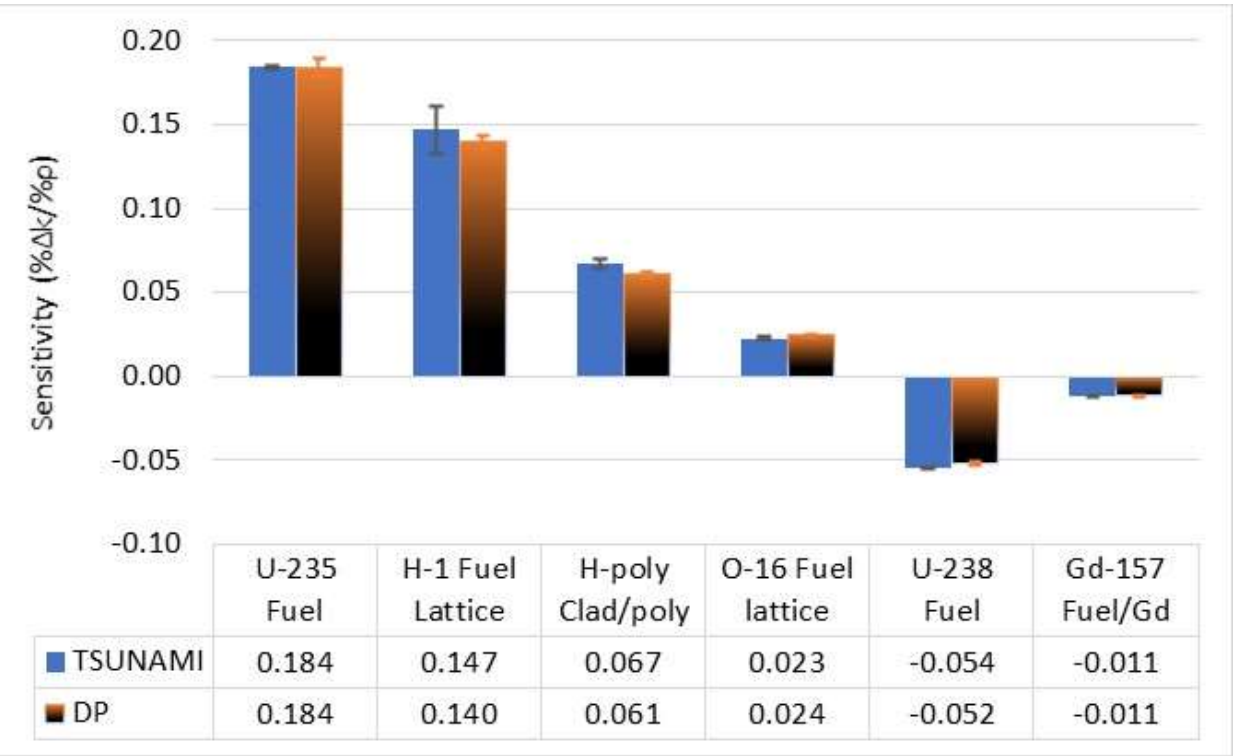

Figure 35. TN-B1 model sensitivity (36 packages, 6.7 wt.\%, $13 \mathrm{Gd}$ rods/fuel assembly).

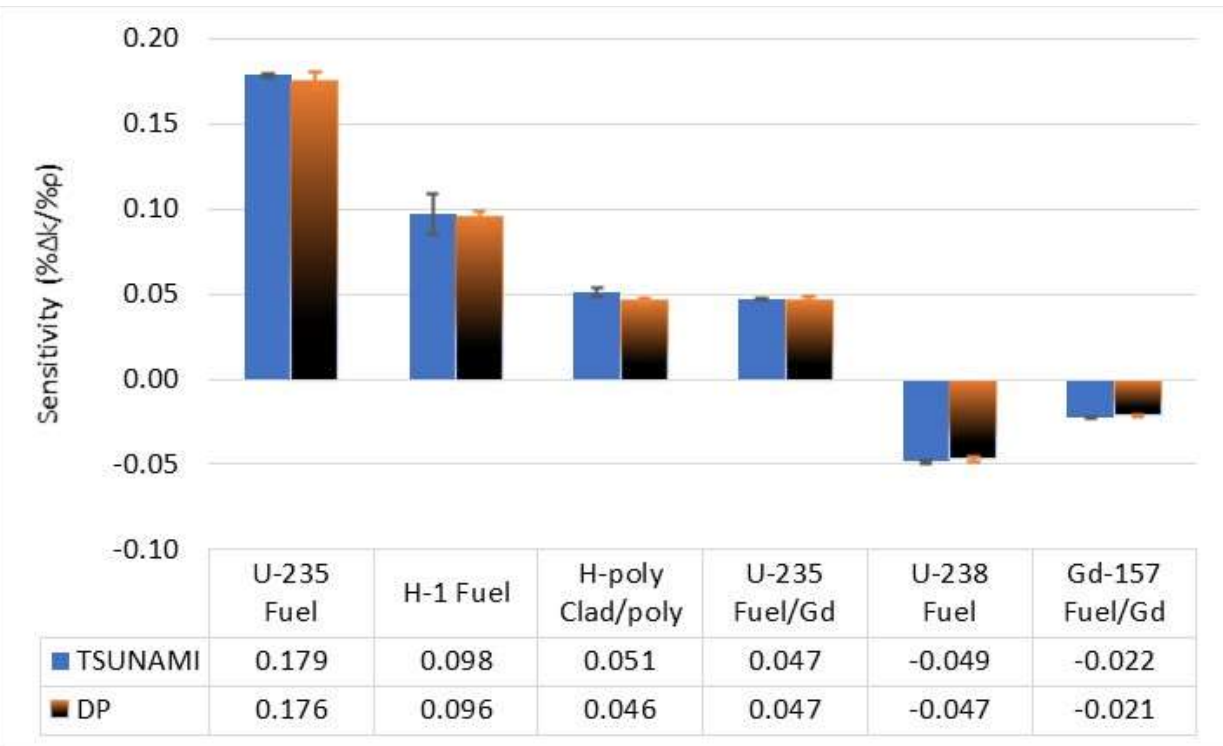

Figure 36. TN-B1 model sensitivity (100 packages, 8 wt.\%, 24 Gd rods/fuel assembly).

Table 24 lists the sensitivity for each mixture in each model. Values greater than $0.01 \% \Delta \mathrm{k} / \% \Sigma$ are highlighted. Sensitivities are similar with $\mathrm{UO}_{2}$ in the non-Gd fuel rods that have the highest sensitivity. The largest difference occurs for lattice/reflector water in the $6 \times 1 \times 6$ array, possibly because the reflector has more importance in a system with more neutron leakage.

Table 24. TN-B1 model sensitivity by mixture.

\begin{tabular}{c|c}
\hline $\begin{array}{c}5 \mathrm{wt} . \%, 13 \mathrm{Gd} \\
\text { rods/assembly, } \\
100 \text { packages }\end{array}$ & $\begin{array}{c}6.7 \mathrm{wt.} \%, 13 \mathrm{Gd} \\
\text { rods/assembly, } \\
36 \text { packages }\end{array}$ \\
\hline
\end{tabular}




\begin{tabular}{|c|c|c|c|c|c|c|c|}
\hline Mixture & Description & Sensitivity & $\begin{array}{c}\text { \% std. } \\
\text { dev. }\end{array}$ & Sensitivity & $\begin{array}{c}\text { \% std. } \\
\text { dev. }\end{array}$ & Sensitivity & $\begin{array}{c}\text { \% std. } \\
\text { dev. }\end{array}$ \\
\hline 1 & $\mathrm{UO}_{2}$ & $\mathbf{0 . 1 5 2}$ & $\mathbf{0 . 4 \%}$ & $\mathbf{0 . 1 3 5}$ & $\mathbf{0 . 5 \%}$ & $\mathbf{0 . 1 3 3}$ & $\mathbf{0 . 5 \%}$ \\
\hline 2 & Clad with polyethylene & $\mathbf{0 . 0 5 6}$ & $\mathbf{2 . 3 \%}$ & $\mathbf{0 . 0 7 2}$ & $\mathbf{1 . 8 \%}$ & $\mathbf{0 . 0 5 3}$ & $\mathbf{2 . 3 \%}$ \\
\hline 4 & $\mathrm{UO}_{2}$ with Gd & 0.000 & $\mathrm{~N} / \mathrm{A}$ & 0.003 & $5.8 \%$ & 0.012 & $2.3 \%$ \\
\hline 6 & $304 \mathrm{SS}$ structure & $\mathbf{- 0 . 0 2 9}$ & $\mathbf{1 . 4 \%}$ & $\mathbf{- 0 . 0 2 1}$ & $\mathbf{1 . 6 \%}$ & $\mathbf{- 0 . 0 2 6}$ & $\mathbf{1 . 7 \%}$ \\
\hline 7 & $\begin{array}{c}\text { Water (lattice and } \\
\text { reflector) }\end{array}$ & $\mathbf{0 . 1 2 4}$ & $\mathbf{5 . 2 \%}$ & $\mathbf{0 . 1 7 0}$ & $\mathbf{4 . 2 \%}$ & $\mathbf{0 . 1 1 2}$ & $\mathbf{5 . 3 \%}$ \\
\hline 8 & Low-density foam & 0.001 & $33.4 \%$ & 0.003 & $12.1 \%$ & 0.003 & $17.6 \%$ \\
\hline 9 & High-density foam & 0.002 & $18.0 \%$ & 0.003 & $11.1 \%$ & 0.003 & $13.8 \%$ \\
\hline 10 & Insulation & 0.009 & $1.5 \%$ & $\mathbf{0 . 0 1 1}$ & $\mathbf{1 . 1 \%}$ & 0.009 & $1.6 \%$ \\
\hline 15 & Zr fuel channel & 0.000 & $28.8 \%$ & 0.002 & $5.9 \%$ & 0.000 & $27.4 \%$ \\
\hline
\end{tabular}

\subsection{TN-B1 Model TSUNAMI-IP Results}

TSUNAMI-IP was used to calculate correlation coefficients $\left(c_{k}\right)$ for the three SDF files in Table 23 and the library of 1,584 critical experiment SDF files. The $5 \mathrm{wt} . \%$ case serves as a reference point for comparison with higher enrichment results. Figures 37, 38, and 39 show the similarity coefficients with different symbols representing different types of experiments.

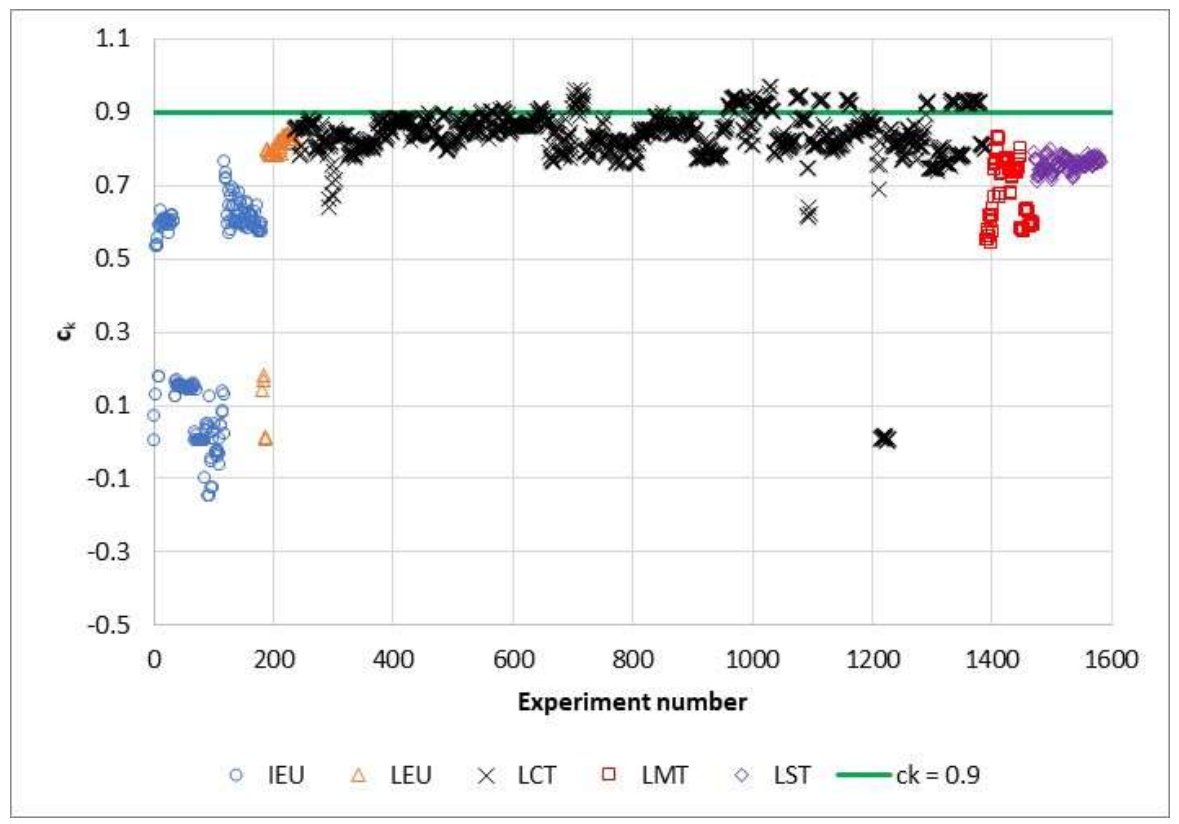

Figure 37. TN-B1 $c_{k}$ for $5 \mathrm{wt.} \% \mathrm{UO}_{2}(100$ packages, $13 \mathrm{Gd}$ rods/fuel assembly). 


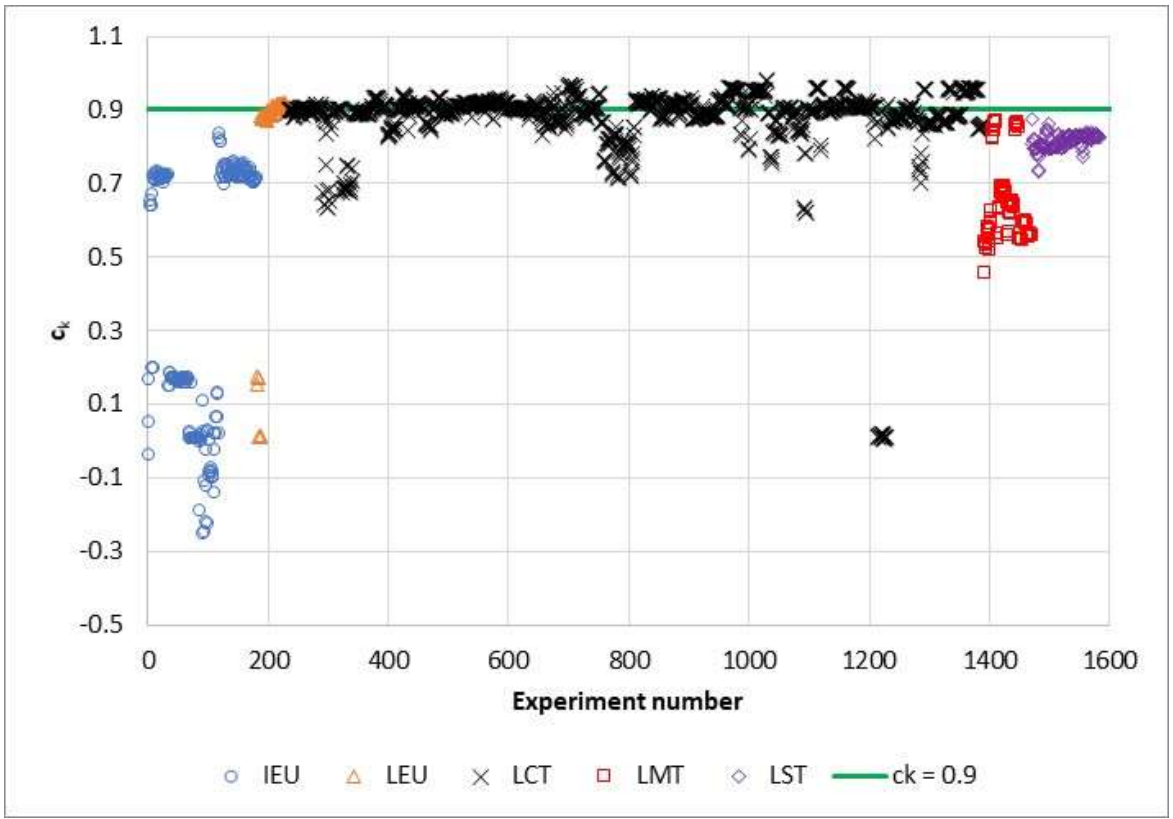

Figure 38 . TN-B1 $c_{k}$ for 6.7 wt.\% $\mathrm{UO}_{2}(36$ packages, $13 \mathrm{Gd}$ rods/fuel assembly).

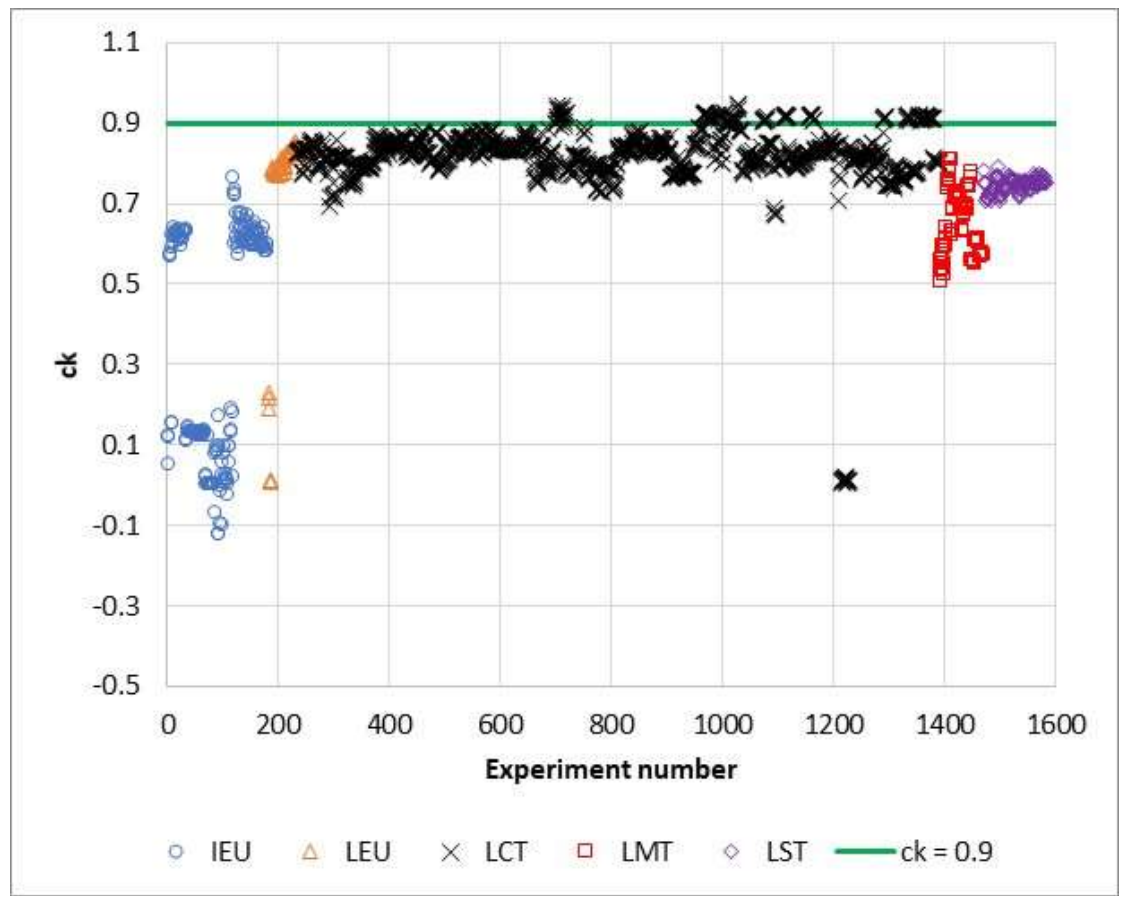

Figure 39. TN-B1 $c_{k}$ for 8 wt.\% $\mathrm{UO}_{2}(100$ packages, $24 \mathrm{Gd}$ rods/fuel assembly).

Table 25 shows the number of experiments in each category with $c_{k} \geq 0.9$ and the median $c_{k}$ for each TNB1model. A full list of experiments with $c_{k}$ values is provided in Appendix A. There are significantly more experiments with $c_{k} \geq 0.9$ for the smaller array (36 packages) with the intermediate enrichment (6.7 wt.\%). This increase in similarity is coincident with the increased importance of lattice and reflector water. There is only a modest difference in similarity results for the 5 and $8 \mathrm{wt} . \% 100$ package models. Overall, numerous experiments are applicable for code validation (marginally similar with $c_{k} \geq 0.8$ or similar with $c_{k} \geq 0.9$ ) for the TN-B1 models with $\mathrm{UO}_{2}$ fuel enrichment up to $8 \mathrm{wt} . \%{ }^{235} \mathrm{U}$. 
Table 25. TN-B1 model TSUNAMI-IP similarity summary.

\begin{tabular}{|c|c|c|c|}
\hline Category & $\begin{array}{c}\mathbf{5} \text { wt.\%, 13 Gd } \\
\text { rods/assembly, } \\
\mathbf{1 0 0} \text { packages }\end{array}$ & $\begin{array}{c}\mathbf{6 . 7} \text { wt.\%, 13 Gd } \\
\text { rods/assembly, } \\
\mathbf{3 6} \text { packages }\end{array}$ & $\begin{array}{c}\mathbf{8} \text { wt.\%, 24 Gd } \\
\text { rods/assembly, } \\
\mathbf{1 0 0} \text { packages }\end{array}$ \\
\hline IEU $c_{k} \geq 0.9$ & 0 & 0 & 0 \\
\hline LEU $c_{k} \geq 0.9$ & 0 & 25 & 0 \\
\hline LCT $c_{k} \geq 0.9$ & 162 & 633 & 0 \\
\hline LMT $c_{k} \geq 0.9$ & 0 & 0 & 0 \\
\hline LST $c_{k} \geq 0.9$ & 0 & 0 & 0.811 \\
\hline Median $c_{k}$ & 0.822 & 0.890 & 0 \\
\hline
\end{tabular}




\section{6. $\mathrm{UF}_{6}$}

\subsection{Daher Nuclear Technologies DN-30 Description}

The DN-30 is a nominally 30 in. diameter 30B cylindrical steel container used for the transportation of up to $2,277 \mathrm{~kg}$ of $\leq 5 \mathrm{wt} . \%$ enriched $\mathrm{UF}_{6}$ per cylinder. Outside the $30 \mathrm{~B}$ is protective structural packaging (PSP) comprised largely of inner and outer steel shells with polyisocyanurate rigid foam between $[13,14]$. Nominal package dimensions are provided in Table 26 . Figure 40 shows the major DN30 components.

Table 26. DN-30 model data.

\begin{tabular}{lc}
\hline \multicolumn{1}{c}{ Parameter } & Value (cm) \\
\hline 30B nominal length & 206.0 \\
\hline 30B nominal diameter & 76.0 \\
\hline 30B wall thickness & 1.3 \\
\hline PSP nominal length & 243.7 \\
\hline PSP nominal diameter & $121.6 \mathrm{c}$ \\
\hline PSP inner shell side thickness & 0.2 \\
\hline PSP outer shell side thickness & 0.3 \\
\hline
\end{tabular}

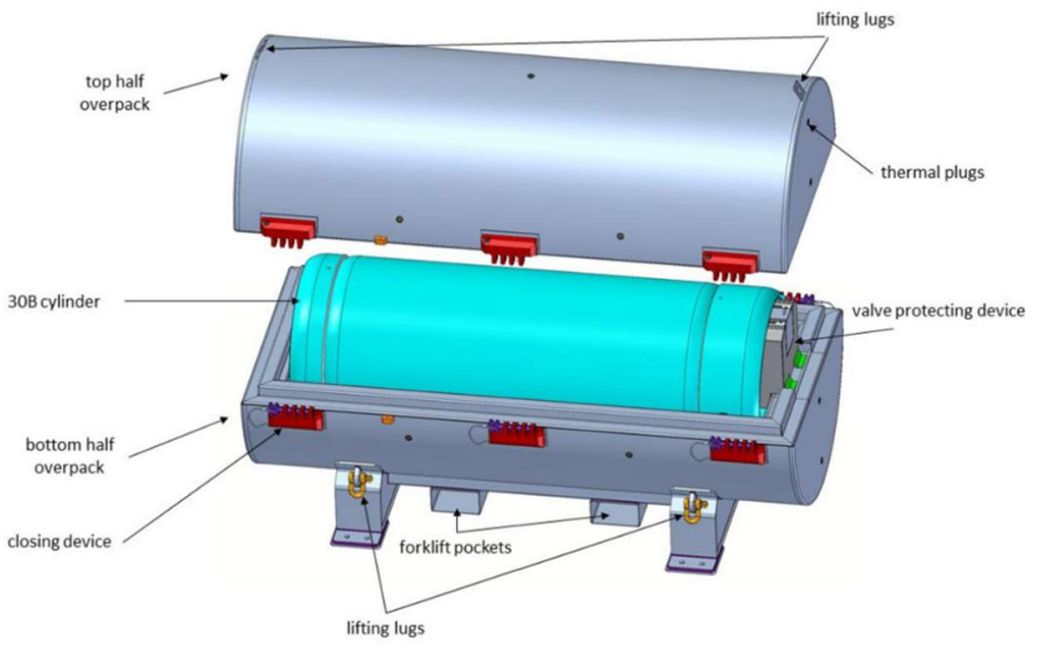

Figure 40. DN-30 package assembly [27].

The most limiting configuration of the DN-30 package identified in the SAR is the HAC infinite package array with a thin cylindrical layer of water between each package in the triangular pitch lattice. Unlike other packages evaluated in this study, $\mathrm{UF}_{6}$ transportation packages that meet certain requirements are exempt from the 10 CFR 71.55(b) assumption of water leakage into the containment system [20]. This exemption applies to $\mathrm{UF}_{6}$ enrichment $\leq 5 \mathrm{wt} . \%$. The SAR most reactive $30 \mathrm{~B}$ contents include $0.5 \mathrm{wt} . \% \mathrm{HF}$ and 3.9-11.4 kg of hydrogenated uranium residues (HUR). $\mathrm{UF}_{6}$ occupies the balance of the internal volume of the $30 \mathrm{~B}$. The minimum internal volume is $0.736 \mathrm{~m}^{3}$, and the maximum allowed $\mathrm{UF}_{6}$ loading is $2,277 \mathrm{~kg}$. The $\mathrm{CoC}$ lists a maximum allowed $\mathrm{H} / \mathrm{U}$ ratio of 0.088 . 
Two 30B models were used in the SAR: one with spherical heads and cylinder skirts on the cylinder ends and one simplified to a right circular cylinder. The maximum OD is $76.8 \mathrm{~cm}$, and the length is $193.5 \mathrm{~cm}$, resulting in a spherical head model internal volume that us almost $10 \%$ greater than the minimum. In this work, a simplified model was used for scoping calculations, and a spherical head model was used for documented calculations.

\subsection{DN-30 SCALE KENO-VI HAC Models}

The DN-30 SAR maximizes the 30B volume by using minimum wall thickness $(0.794 \mathrm{~cm})$ and maximal cylinder dimensions. The PSP is assumed to collapse down onto the 30B with only the PSP SS walls $(0.5 \mathrm{~cm}$ thick radially and $1.4 \mathrm{~cm}$ thick axially) and a water layer for optimum moderation between packages.

Four aspects of the SAR HAC model are highly important for criticality calculations:

1) the thickness and placement (inside or outside the collapsed PSP shells) of a water layer outside the 30B;

2) the presence of the collapsed PSP shells;

3) a three-shell spherical model of the HUR with HUR in the center, a shell of $\mathrm{UF}_{6}$, and a shell of HF; and

4) the position of the HUR sphere.

The nonproprietary SAR does not provide details to allow the SAR HAC model to be replicated. The DN30 model used in this work includes the important features and produces very similar $k_{\text {eff }}$ with $5 \mathrm{wt} . \%$ $\mathrm{UF}_{6}$. The SAR infinite array HAC $k_{\text {eff }}$ is 0.935 , including 3 sigma uncertainty. The SAR model has $5.5 \mathrm{~g} / \mathrm{cc} 99.5 \mathrm{wt} . \% \mathrm{UF}_{6}$ and $0.5 \mathrm{wt} . \%$ HF filling the volume not occupied by HUR. For the same conditions, $k_{\text {eff }}$ that uses the KENO-VI model developed herein is 0.935 (no uncertainty). This model includes $11.4 \mathrm{~kg}$ of HUR of the form $\mathrm{UO}_{2} \mathrm{~F}_{2} * 3 \mathrm{H}_{2} \mathrm{O}$ with a density of $6.3 \mathrm{~g} / \mathrm{cc}$ [21].

The SAR bounding material orientation is a complex arrangement of $\mathrm{UF}_{6}$ and a sphere of $\mathrm{UF}_{6}, \mathrm{HF}$, and HUR (multiple spherical layers) placed near one end of the cylinder. Scoping calculations confirmed that modeling the HUR as a multilayer sphere produces higher $k_{\text {eff }}$ than homogenizing the HUR with $\mathrm{UF}_{6}$. The SAR indicated maximum $\mathrm{UF}_{6}$ density with no void space in the cylinder maximized $k_{\text {eff. }}$.

Other work suggests that $k_{\text {eff }}$ is maximum at reduced $\mathrm{UF}_{6}$ density [22]. Scoping calculations with and without the HUR sphere in a $5 \mathrm{wt} . \%$ DN-30 model show that the HUR sphere increases $k_{\text {eff }}$ significantly and reverses the trend with $\mathrm{UF}_{6}$ density (Figure 41). The HUR is neutronically important because HUR spheres are positioned to maximize interactions between HUR spheres in adjacent cylinders and because the HUR sphere contains much more moderator than the larger volume of $\mathrm{UF}_{6}$. Figure 42 shows the HUR sphere positioning in a KENO-VI $3 \times 2 \mathrm{HAC}$ array model. 


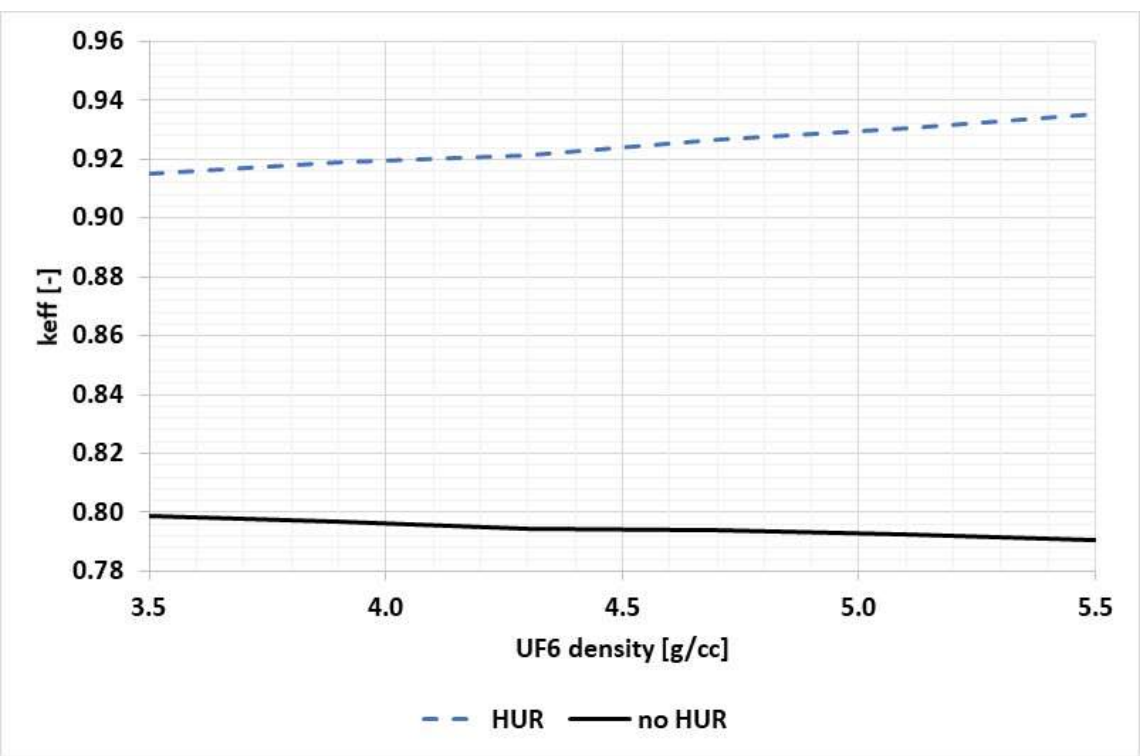

Figure 41. Effect of HUR on $\boldsymbol{k}_{\text {eff }}$.

The enrichment of the U in HUR is important. Increased $U_{6} F_{6}$ enrichment will lead to increased HUR enrichment. For this work, HUR enrichment is assumed to be the same as $\mathrm{UF}_{6}$ enrichment. In practice, the HUR enrichment might need to be modeled as the highest enrichment that a cylinder is licensed to transport.

Sampler was used to perform $k_{\text {eff }}$ calculations over a range of $\mathrm{UF}_{6}$ density and water layer thickness. Figure 43 shows $k_{\text {eff }}$ results for a $3 \times 2 \mathrm{HAC}$ array with $6.7 \mathrm{wt} \% \%$ enriched $\mathrm{UF}_{6}$. Maximum reactivity occurs with maximum $\mathrm{UF}_{6}$ density and a $0.4 \mathrm{~cm}$ water layer around each $30 \mathrm{~B}$ cylinder. Several combinations of enrichment and HAC array size were investigated. Unlike the array models, the highenrichment single cylinder model had maximum reactivity with $1.4 \mathrm{~cm}$ of water between the $30 \mathrm{~B}$ and the PSP steel. For that single package model, increased water layer thickness moves the PSP steel further from the $\mathrm{UF}_{6}$ but does not increase the distance between packages. 

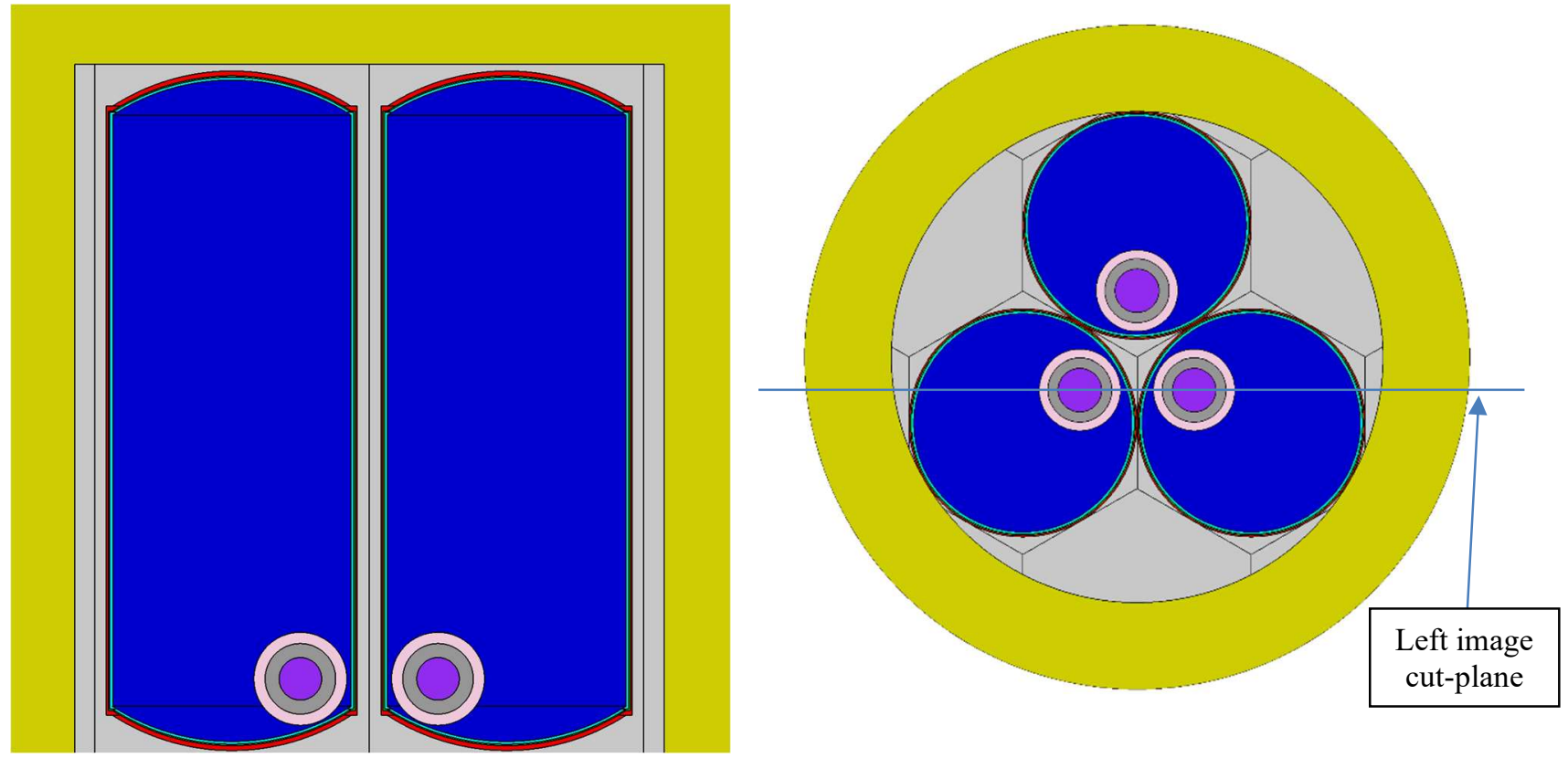

Figure 42. DN-30 $3 \times 2$ HAC array model cutaway views.

\subsection{DN-30 Criticality Calculations}

Criticality calculations were performed to determine combinations of enrichment and array size with $k_{\text {eff }}$ approximately equal to the $5 \mathrm{wt}$ \% infinite array model. Sampler was used to determine the most reactive water layer thickness and $\mathrm{UF}_{6}$ density. Figures 43 and 44 show the Sampler results for the $3 \times 2$ and single package models, respectively. Table 27 shows the combinations of enrichment and array size with $k_{\text {eff }}$ near 0.935 . The trend of enrichment with inverse HAC array size (1/number of packages) is shown in Figure 45. Subcriticality with no water flooding of the 30B interior can be maintained with increased enrichment by reducing the maximum HAC array size (increasing CSI) up to $12.5 \mathrm{wt} . \%$. These results rely partly on the SAR modeling approach, particularly the treatment of HUR. The criticality calculations presented provide useful insights but should not be considered exhaustive. 


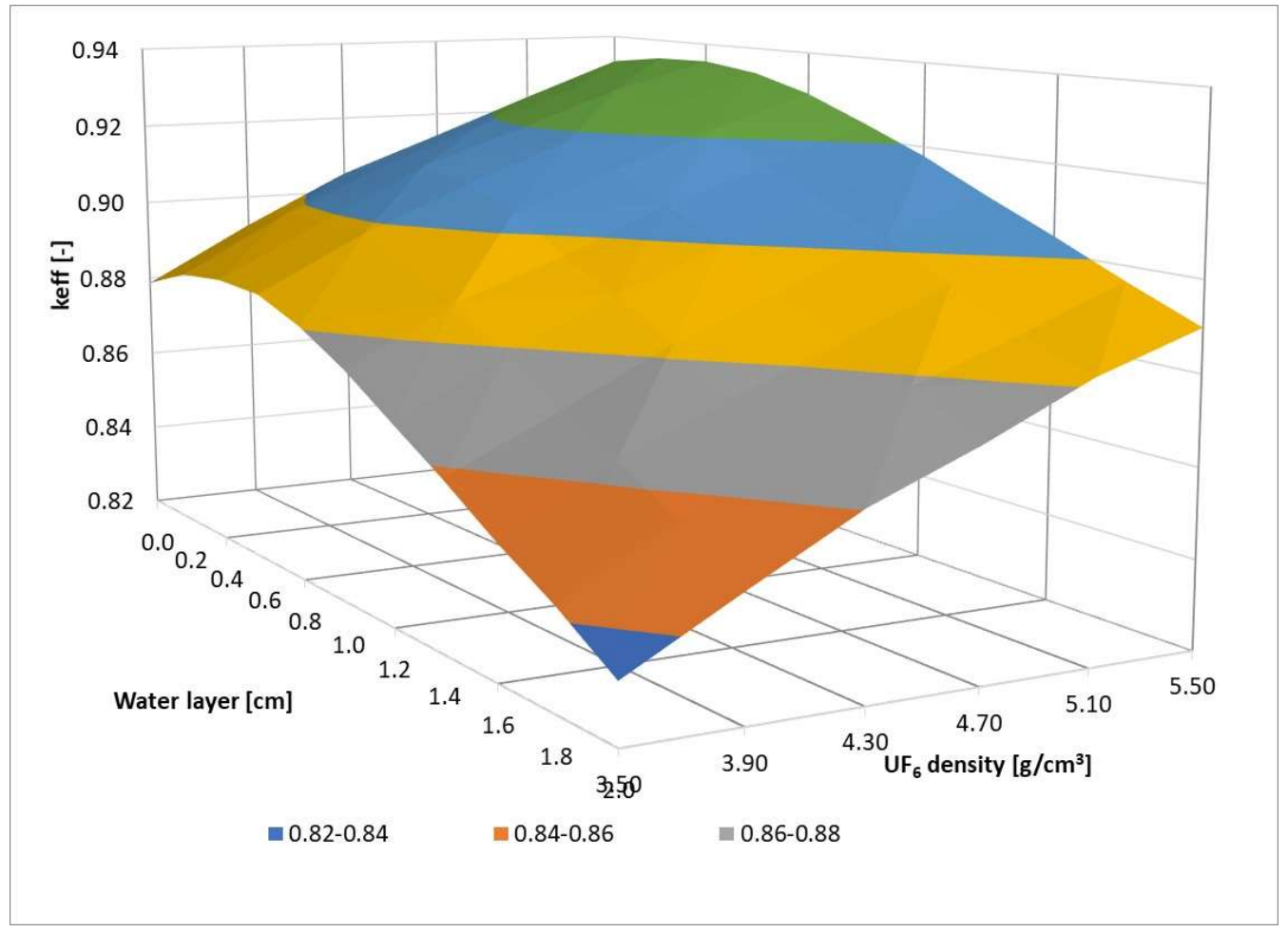

Figure 43. DN-30 3 × 2 HAC array $k_{\text {eff }}$.

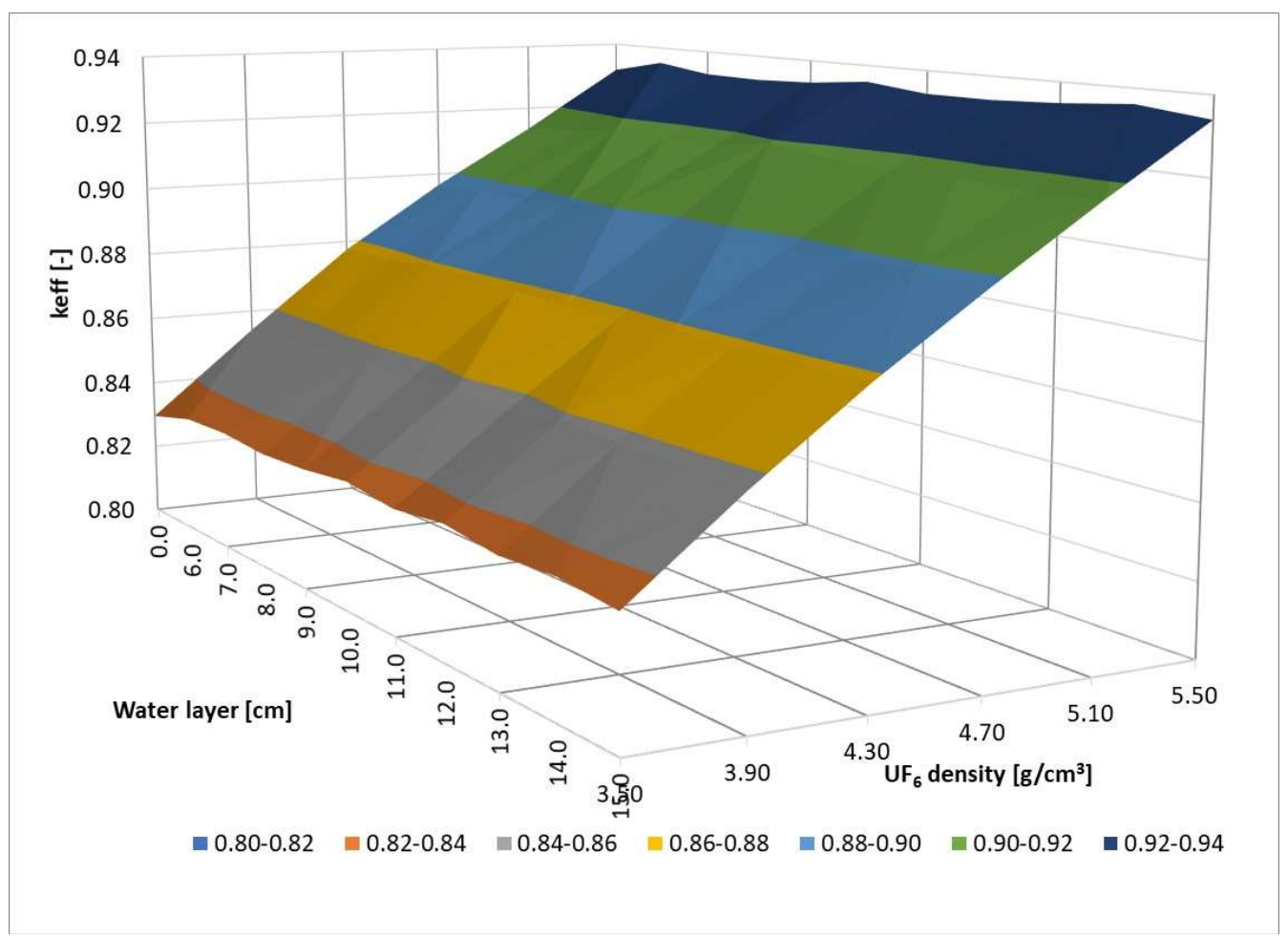

Figure 44. DN-30 HAC single package $k_{e f f}$. 
Table 27. DN-30 model enrichment and array size.

\begin{tabular}{|c|c|c|c|c|}
\hline $\begin{array}{c}\text { Enrichment } \\
\left(\mathbf{w t . \%} \mathbf{~}^{\mathbf{2 3 5}} \mathbf{U}\right)\end{array}$ & HAC array size & 1/array size & $\boldsymbol{k}_{\text {eff }}$ & EALF (eV) \\
\hline 5 & Infinite & 0 & $0.9353 \pm 0.00048$ & 45.2 \\
\hline 5.8 & $7 \times 2$ & 0.1 & $0.9354 \pm 0.00049$ & 36.5 \\
\hline 6.7 & $3 \times 2$ & 0.2 & $0.9350 \pm 0.00048$ & 33.2 \\
\hline 9.5 & $1 \times 2$ & 0.5 & $0.9350 \pm 0.00048$ & 69.4 \\
\hline 12.5 & $1 \times 1$ & 1.0 & $0.9360 \pm 0.00048$ & 123.9 \\
\hline
\end{tabular}

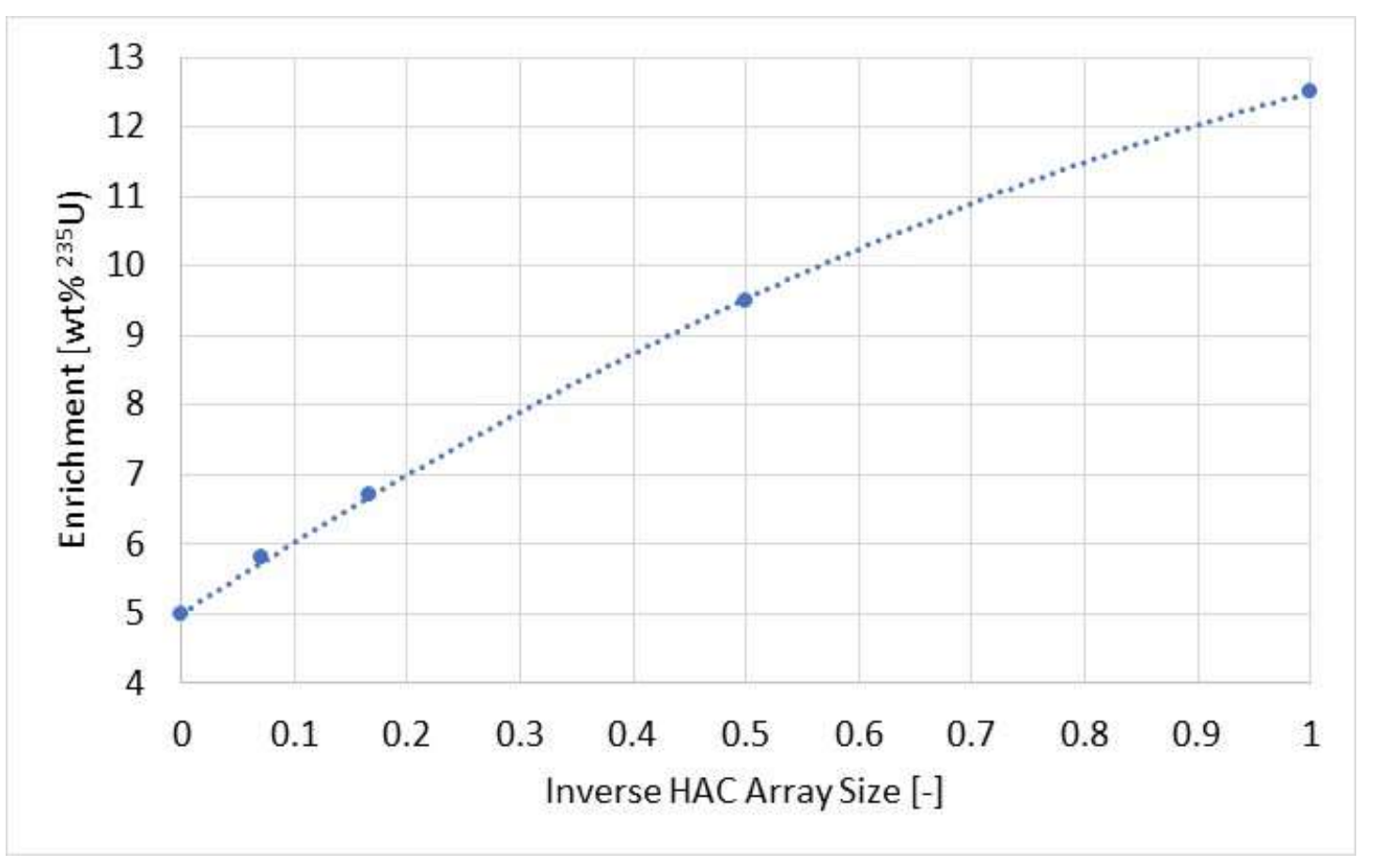

Figure 45. DN-30 model enrichment and array size.

\subsection{DN-30 TSUNAMI-3D Calculations}

TSUNAMI-3D S/U calculations were performed for three cases from Table 27. Table 28 shows key TSUNAMI input values, forward and adjoint $k_{\text {eff }}$, the energy of the average lethargy of fission, and total cross section uncertainty. 
Table 26. DN-30 HAC model TSUNAMI-3D case data.

\begin{tabular}{|c|c|c|c|}
\hline Parameter & $\begin{array}{c}\mathbf{5 . 0} \text { wt.\%, infinite package } \\
\text { array }\end{array}$ & $\begin{array}{c}\mathbf{6 . 7} \mathbf{w t . \% , 3 \times 2} \text { package } \\
\text { array }\end{array}$ & $\mathbf{1 2 . 5}$ wt.\%, single package \\
\hline Grid mesh & $40 \times 40 \times 55$ & $46 \times 46 \times 36$ & $46 \times 46 \times 56$ \\
\hline Particles/gen. & $50,000 \mathrm{fwd} . / 300,000 \mathrm{adj}$. & $50,000 \mathrm{fwd} . / 250,000 \mathrm{adj}$. & $50,000 \mathrm{fwd} . / 300,000 \mathrm{adj}$. \\
\hline SDF file name & $\mathrm{T} 3 \mathrm{D} \_\mathrm{dn} 30 \mathrm{hac}$ inf_5wt & T3D_dn30_hac_3x2_6-7wt & T3D_dn30_hac_sgl_12-5wt \\
\hline Forward $k_{\text {eff }}$ & $0.93513 \pm 0.00031$ & $0.93542 \pm 0.00029$ & $0.93229 \pm 0.00049$ \\
\hline Adjoint $k_{\text {eff }}$ & $0.9333 \pm 0.0014$ & $0.9362 \pm 0.0014$ & $0.9346 \pm 0.0014$ \\
\hline EALF & $51.5 \mathrm{eV}$ & $34.5 \mathrm{eV}$ & $155.1 \mathrm{eV}$ \\
\hline $\begin{array}{c}\text { Cross section } \\
\text { uncertainty }\end{array}$ & $0.660 \% \Delta \mathrm{k} / \mathrm{k}$ & $0.676 \% \Delta \mathrm{k} / \mathrm{k}$ & $0.915 \% \Delta \mathrm{k} / \mathrm{k}$ \\
\hline
\end{tabular}

The adequacy of the TSUNAMI input is confirmed by comparing TSUNAMI-calculated nuclide sensitivity with KENO DP sensitivity. Figures 46, 47, and 48 summarize the sensitivity results calculated by each method for the highest sensitivity nuclide/region combinations. There is good agreement between the DP and TSUNAMI sensitivities, which confirms that the TSUNAMI SDFs properly represent the models. HUR is a high-sensitivity material in all three models.

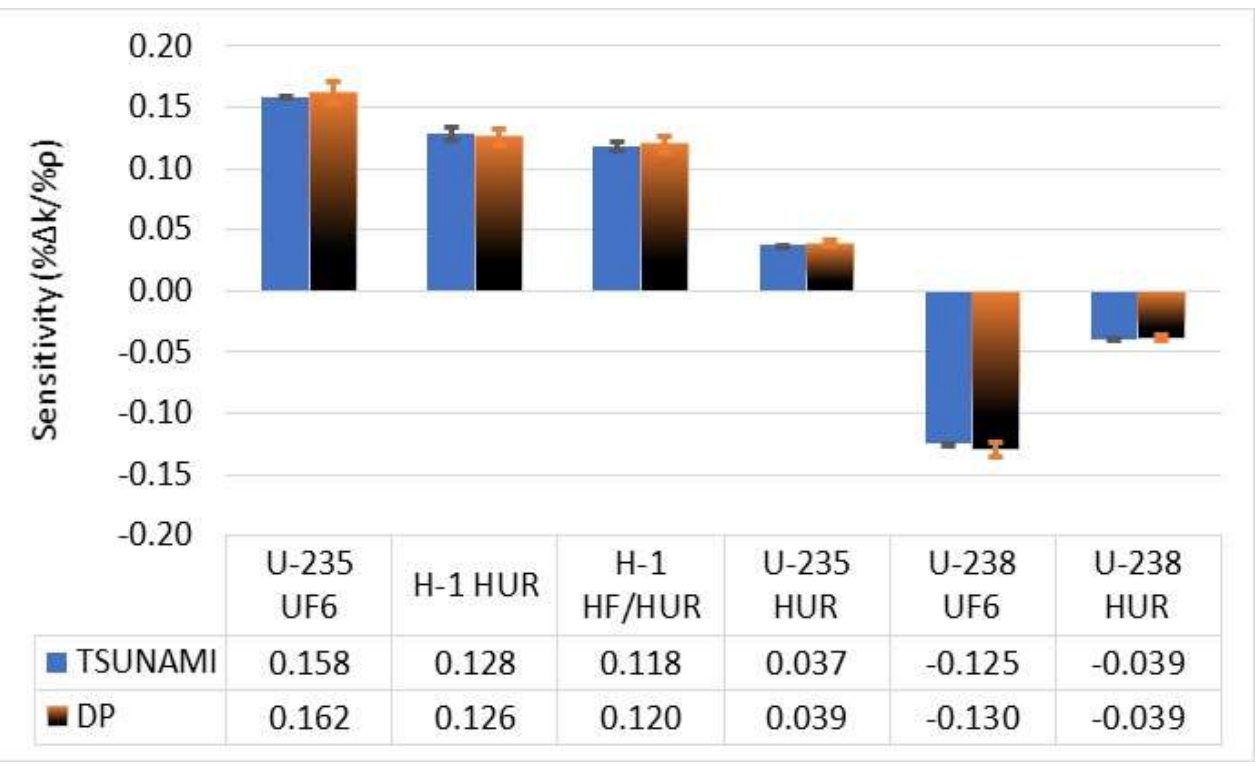

Figure 46. DN-30 model sensitivity (5 wt.\%, infinite array). 


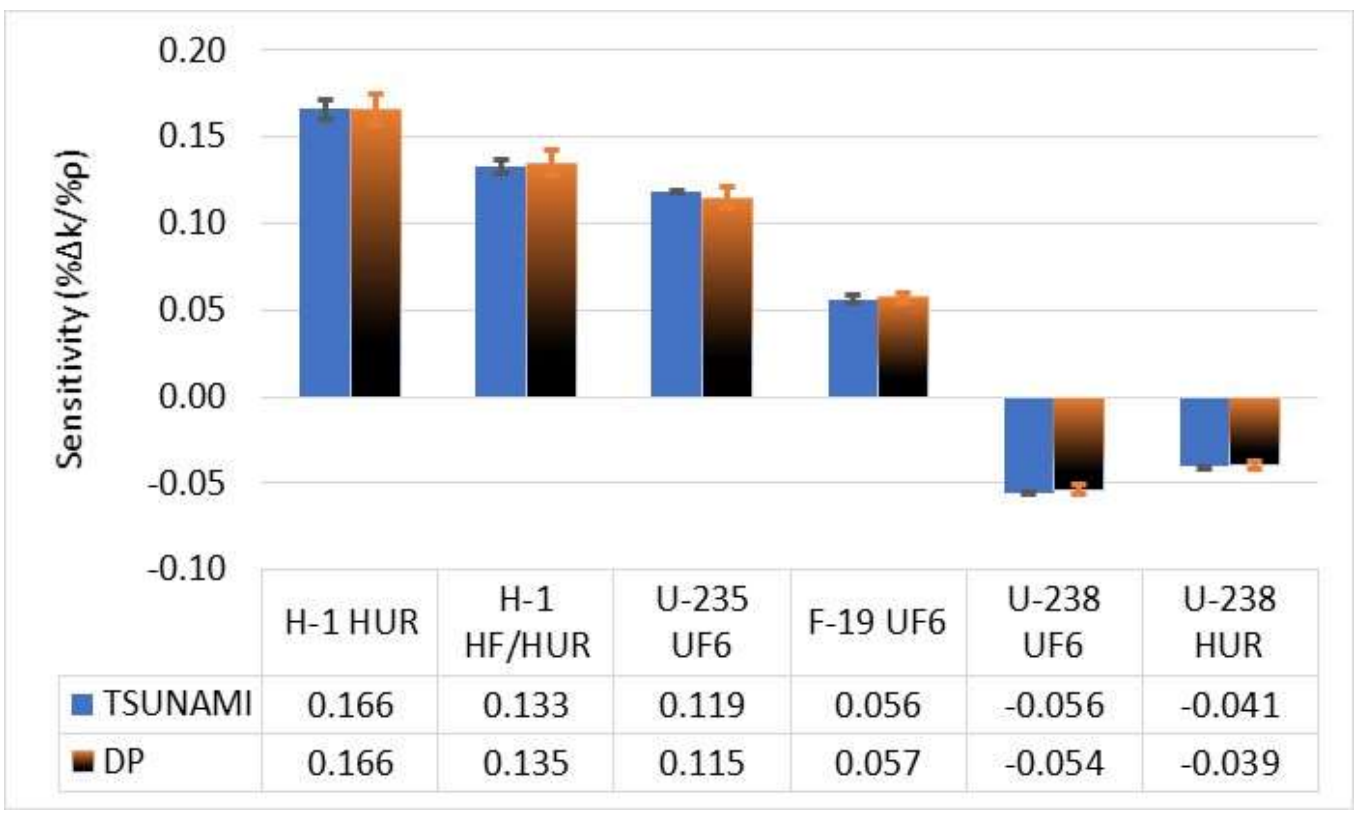

Figure 47. DN-30 model sensitivity $(6.7 \mathrm{wt.} \%, 3 \times 2$ array $)$.

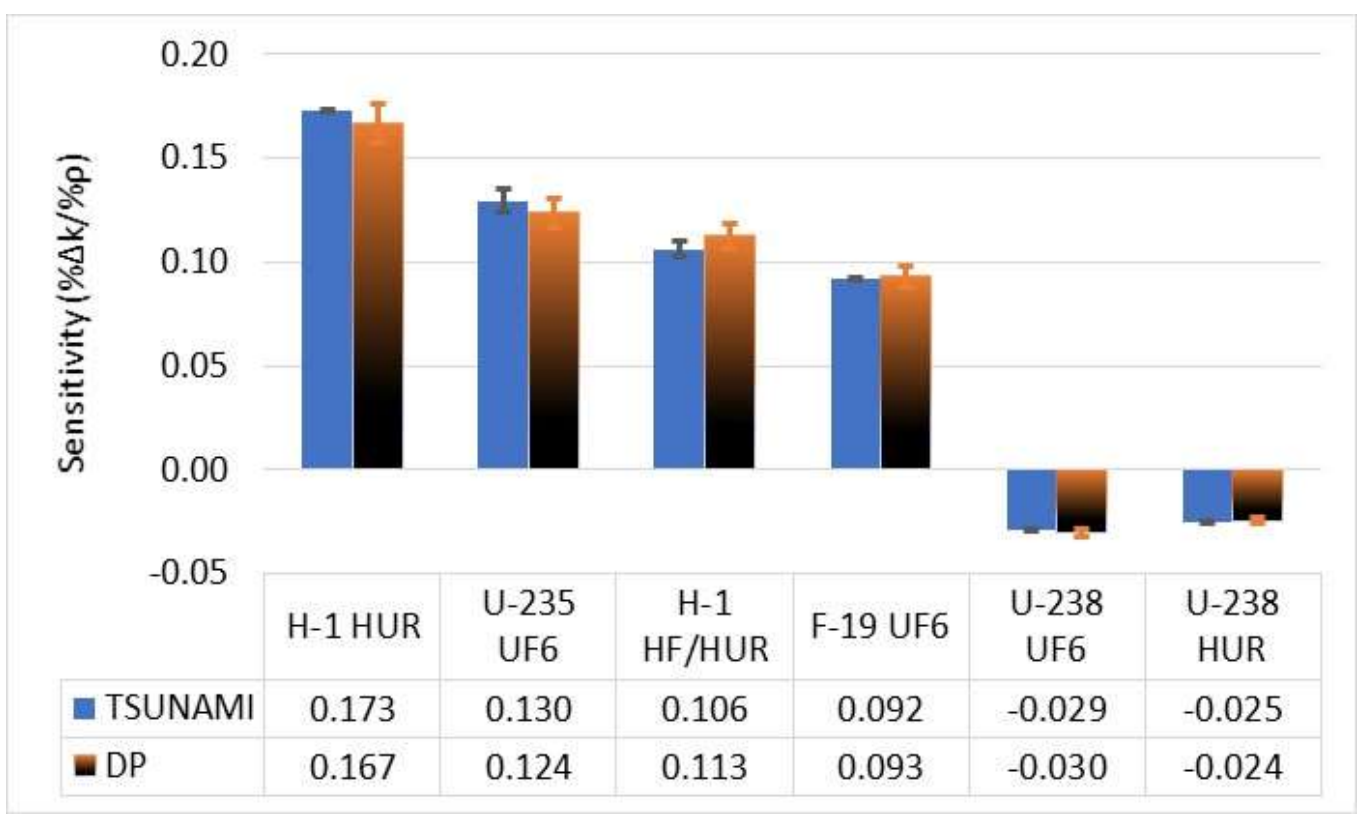

Figure 48. DN-30 model sensitivity (12.5 wt.\%, single package).

Table 29 lists the sensitivity for each mixture in each model. Values greater than $0.01 \% \Delta \mathrm{k} / \% \Sigma$ are highlighted. The importance of HUR stands out (mixtures 6, 7, and 8). The importance of moderation provided by the $0.5 \mathrm{wt} \% \mathrm{HF}$ impurity in the $\mathrm{UF}_{6}$ increases strongly with increasing enrichment (mixture 1). 
Table 27. DN-30 model sensitivity by mixture.

\begin{tabular}{|c|c|c|c|c|c|c|c|}
\hline \multirow[b]{2}{*}{ Mixture } & \multirow[b]{2}{*}{ Description } & \multicolumn{2}{|c|}{$\begin{array}{l}5.0 \text { wt. } \% \text {, infinite } \\
\text { package array }\end{array}$} & \multicolumn{2}{|c|}{$\begin{array}{l}6.7 \mathrm{wt} . \%, 3 \times 2 \\
\text { package array }\end{array}$} & \multicolumn{2}{|c|}{$\begin{array}{c}12.5 \text { wt. } \% \text {, single } \\
\text { package }\end{array}$} \\
\hline & & Sensitivity & $\begin{array}{c}\text { \% std. } \\
\text { dev. }\end{array}$ & Sensitivity & $\begin{array}{c}\text { \% std. } \\
\text { dev. }\end{array}$ & Sensitivity & $\begin{array}{c}\text { \% std. } \\
\text { dev. }\end{array}$ \\
\hline 1 & $\mathrm{UF}_{6} / \mathrm{HF}$ & 0.040 & $3.0 \%$ & 0.127 & $0.8 \%$ & 0.205 & $0.5 \%$ \\
\hline 2 & 30B steel & -0.023 & $1.7 \%$ & -0.016 & $1.7 \%$ & -0.018 & $1.2 \%$ \\
\hline 3 & $\mathrm{H}_{2} \mathrm{O}$ layer & 0.004 & $15.6 \%$ & 0.004 & $15.4 \%$ & 0.016 & $16.0 \%$ \\
\hline 4 & $\mathrm{H}_{2} \mathrm{O}$ reflector & $\mathrm{N} / \mathrm{A}$ & $\mathrm{N} / \mathrm{A}$ & 0.000 & $\mathrm{~N} / \mathrm{A}$ & 0.000 & $\mathrm{~N} / \mathrm{A}$ \\
\hline 5 & PSP 304 SS & -0.018 & $1.7 \%$ & -0.005 & $3.7 \%$ & 0.000 & $\mathrm{~N} / \mathrm{A}$ \\
\hline 6 & HUR & 0.136 & $2.0 \%$ & 0.178 & $1.7 \%$ & 0.196 & $1.5 \%$ \\
\hline 7 & $\mathrm{UF}_{6} / \mathrm{HF}$ (HUR) & 0.016 & $4.2 \%$ & 0.027 & $2.6 \%$ & 0.042 & $1.6 \%$ \\
\hline 8 & HF (HUR) & 0.128 & $1.6 \%$ & 0.146 & $1.3 \%$ & 0.123 & $1.3 \%$ \\
\hline
\end{tabular}

\subsection{DN-30 Model TSUNAMI-IP Results}

TSUNAMI-IP was used to calculate correlation coefficients $\left(c_{k}\right)$ for the three SDF files in Table 28 and the library of 1,584 critical experiment SDF files. The $5 \mathrm{wt} . \%$ case serves as a reference point for comparison with higher enrichment results. Figures 49, 50, and 51 show the similarity coefficients with different symbols representing different types of experiments.

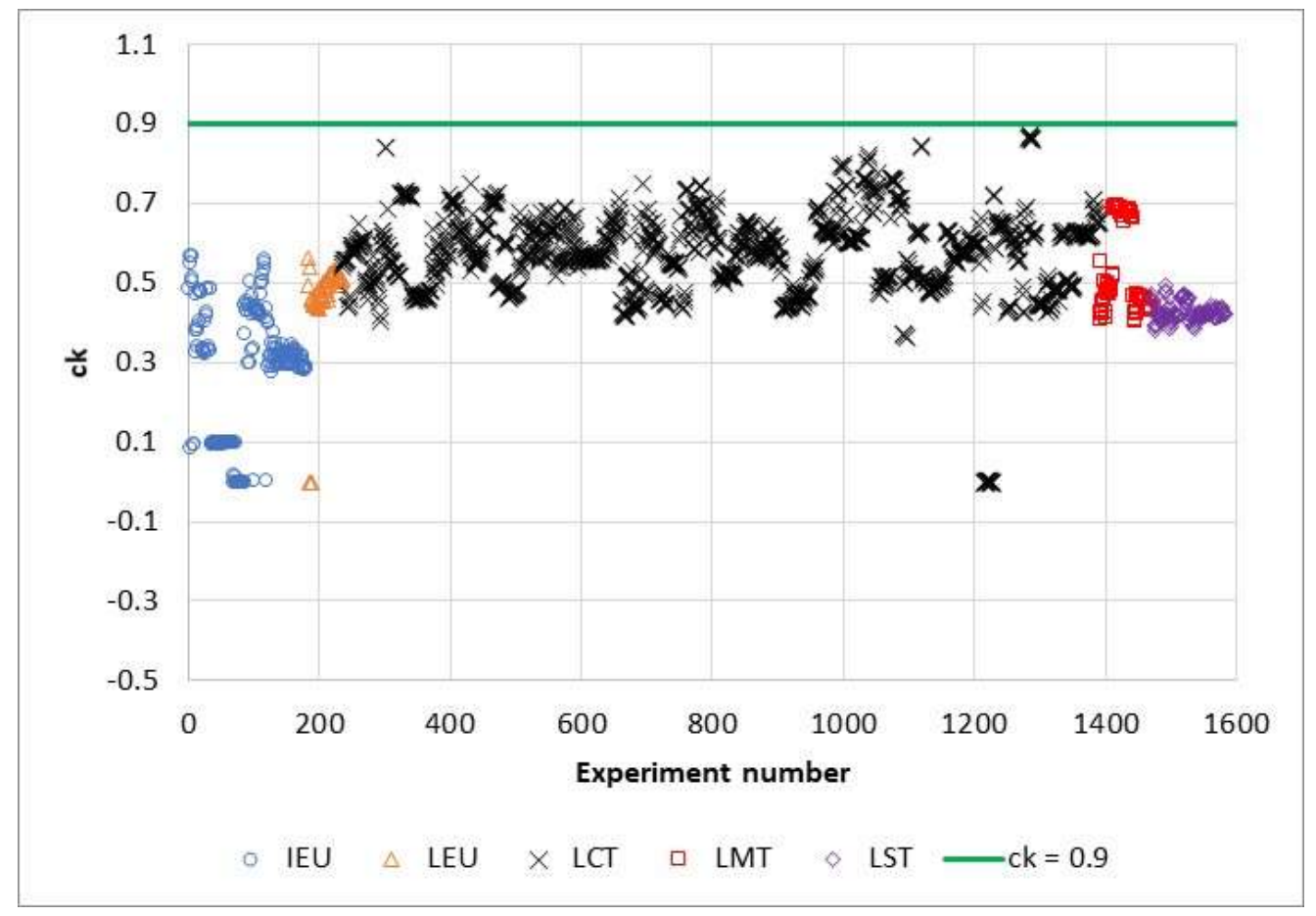

Figure 49. DN-30 $c_{k}$ for 5 wt.\% UF (infinite HAC array). 


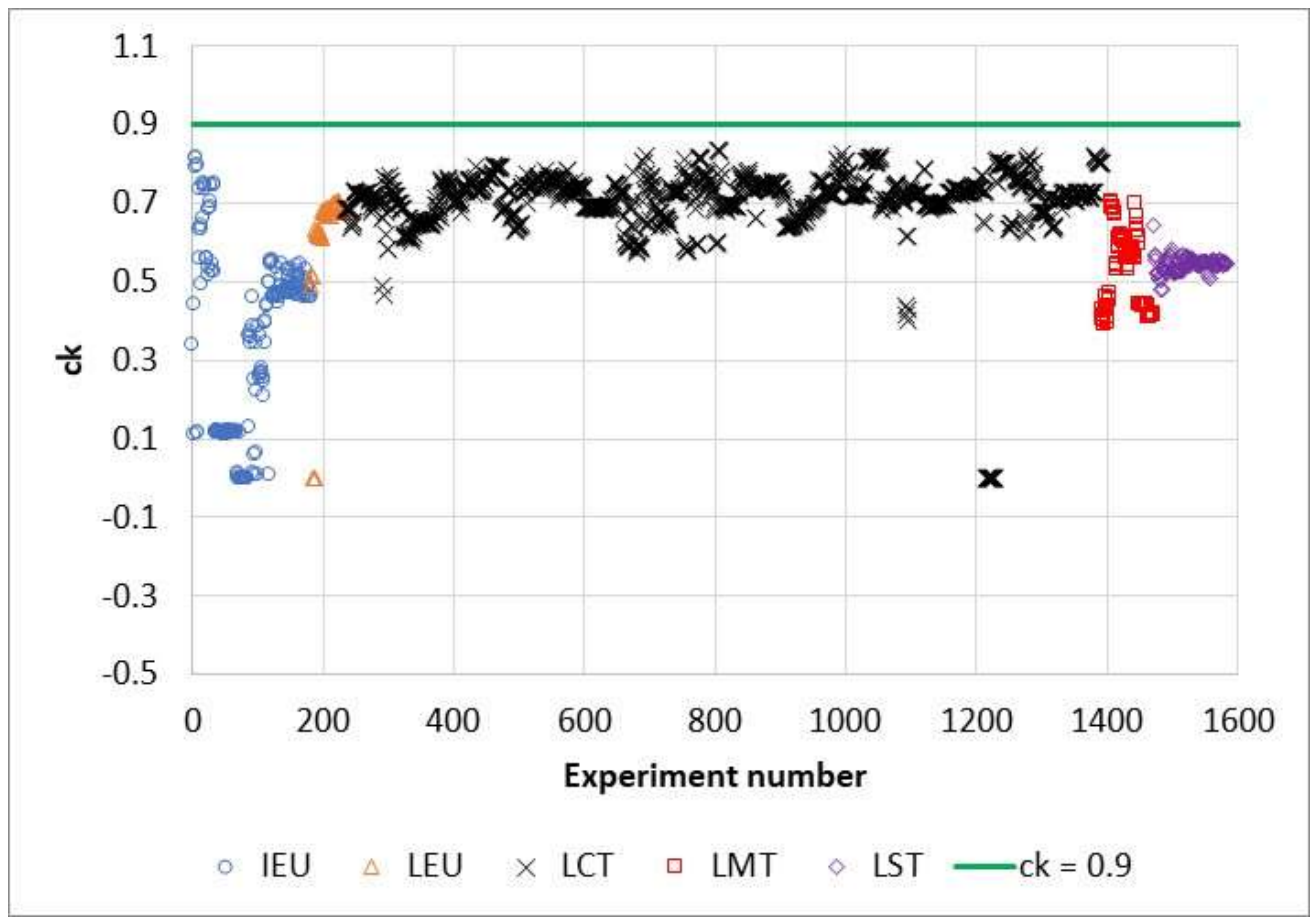

Figure 40. DN-30 $c_{k}$ for $6.7 \mathrm{wt} . \% \mathrm{UF}_{6}(3 \times 2 \mathrm{HAC}$ array $)$.

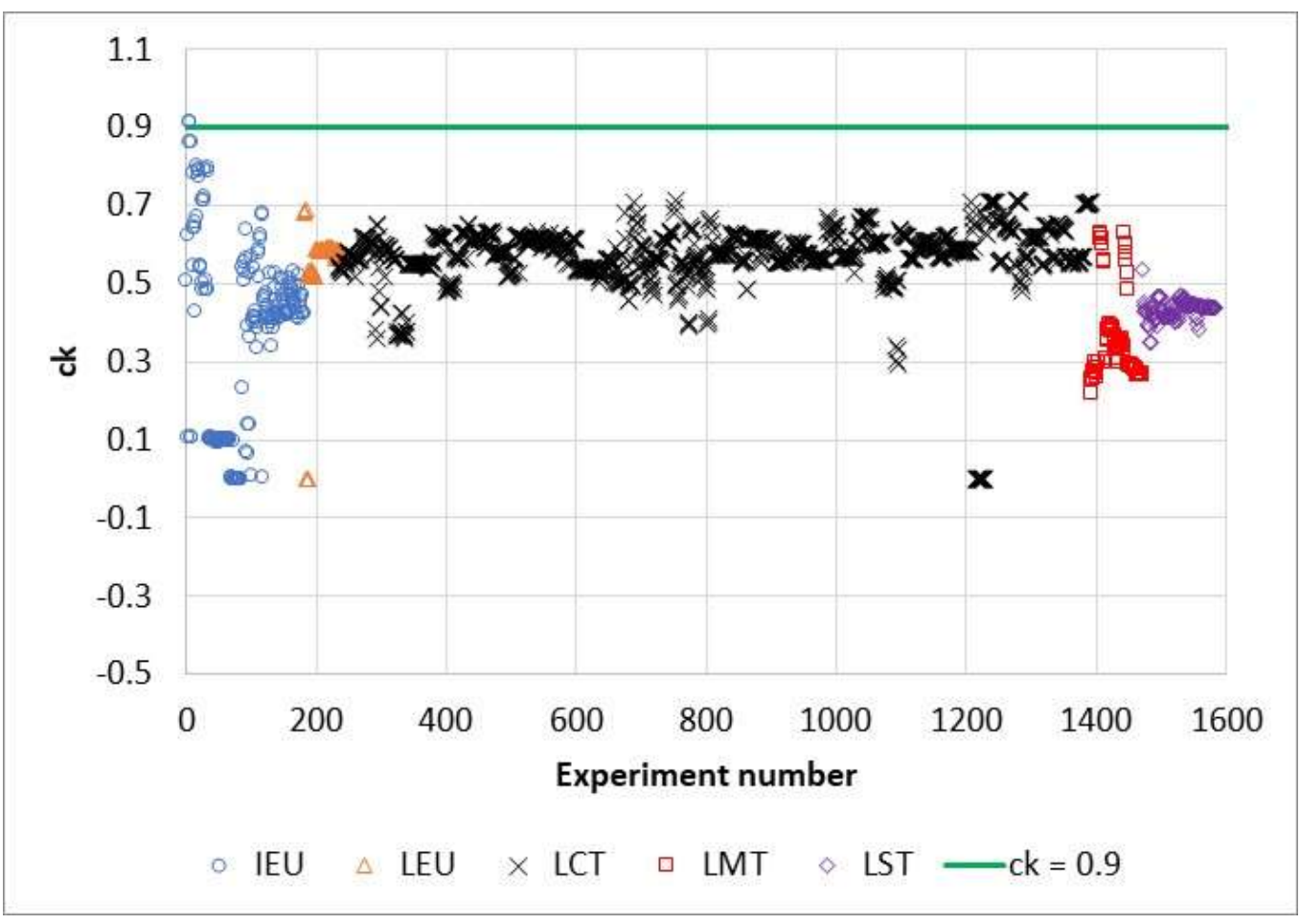

Figure 41. DN-30 $c_{k}$ for $12.5 \mathrm{wt} . \% \mathrm{UF}_{6}$ (single package). 
Table 30 shows the number of experiments in each category with $c_{k} \geq 0.9$ and the median $c_{k}$ for each of the DN-30 models. A full list of experiments with $c_{k}$ values is provided in Appendix A. There are only two experiments with $c_{k} \geq 0.9$ for any of the three DN-30 models. Only the $3 \times 26.7 \mathrm{wt} . \%$ model has a reasonable number of validation candidates (66) with $c_{k} \geq 0.8$. The median $c_{k}$ is also much higher for that enrichment/array size combination. Similarity decreases with increasing enrichment and increases with decreasing array size. Appendix $\mathrm{C}$ has an expanded discussion of the trend of similarity with array size.

Table 30. DN-30 model TSUNAMI-IP similarity summary.

\begin{tabular}{|c|c|c|c|}
\hline Category & $\begin{array}{c}\mathbf{5 . 0} \text { wt.\%, infinite } \\
\text { package array }\end{array}$ & $\begin{array}{c}\mathbf{6 . 7} \text { wt.\%, } \mathbf{3} \times \mathbf{2} \text { package } \\
\text { array }\end{array}$ & $\begin{array}{c}\mathbf{1 2 . 5} \text { wt.\%, single } \\
\text { package }\end{array}$ \\
\hline IEU $c_{k} \geq 0.9$ & 0 & 0 & 2 \\
\hline LEU $c_{k} \geq 0.9$ & 0 & 0 & 0 \\
\hline LCT $c_{k} \geq 0.9$ & 0 & 0 & 0 \\
\hline LMT $c_{k} \geq 0.9$ & 0 & 0 & 0 \\
\hline LST $c_{k} \geq 0.9$ & 0 & 0 & 0 \\
\hline IEU $c_{k} \geq 0.8$ & 0 & 2 & 5 \\
\hline LEU $c_{k} \geq 0.8$ & 0 & 0 & 0 \\
\hline LCT $c_{k} \geq 0.8$ & 15 & 66 & 0 \\
\hline LMT $c_{k} \geq 0.8$ & 0 & 0 & 0 \\
\hline LST $c_{k} \geq 0.8$ & 0 & 0 & 0 \\
\hline Median $c_{k}$ & 0.548 & 0.699 & 0.567 \\
\hline
\end{tabular}

The lack of experiments with a $c_{k}$ value in excess of 0.8 does not mean that a validation is impossible; a validation must be performed for all computed analysis results. The lack of highly applicable experiments indicates that the bias resulting from these benchmarks might not be highly representative of the bias for the application system. The solution to this is to apply an additional reactivity margin to ensure that the aggregate margin included in the bias, bias uncertainty, and additional margin conservatively represents the bias that is expected for the application. $\mathrm{S} / \mathrm{U}$ techniques provide tools for generating a quantitative, defensible estimate of what an appropriate margin might be.

Examining the nuclides and reactions that contribute significantly to data-induced uncertainty will indicate the important processes to be validated. Some elements, such as F, might be entirely absent from the validation set or only poorly represented. For these nuclides, the data-induced uncertainty can provide an estimate for the magnitude of the bias that occurs in the application. This approach is essentially the same as that used in several other works $[23,24,25]$.

In other cases, important nuclides will be present in the validation suite, but the energy-dependent sensitivity profiles will differ significantly. These profiles can be reviewed to determine whether validation exists for an energy range or perhaps multiple energy ranges. For example, it might be evident that high-energy cross sections are validated because fission neutrons are born at high energies in all systems. Thermal cross sections are generally well-validated due to the many available thermal benchmark experiments. Therefore, an estimate of the unvalidated portion of the profile can be generated and used to estimate the magnitude of the remaining potential bias in the application in the energy ranges with weaker validation. In some cases, an energy range in the application might have significantly less sensitivity than is present in relevant benchmarks. An additional margin for these situations is likely not needed because the low sensitivity directly indicates a low potential for bias. The margin estimates generated for the partially validated nuclides are likely to be less precise than those resulting from an entirely unrepresented nuclide and thus should be developed with some deliberate conservatism. 
Overall, this approach is similar to the PENALTY assessment in TSUNAMI-IP [26], but it does not necessarily give credit for single experiments that cover an energy range. This option might still be useful as a reference value. The magnitude of the data-induced uncertainty in the application also provides a bound on the estimated magnitude of the bias given no applicable benchmarks. Using this value might be more efficient than investing effort in developing and defending a lower additional margin. 


\section{CONCLUSIONS}

A criticality assessment of five representative transportation packages was performed to determine whether existing package designs can be used to transport unirradiated increased enrichment (within the HALEU range) LWR fuel materials of different forms. The following representative packages were evaluated:

1) Traveller (PWR fuel assemblies, PWR and BWR fuel pins),

2) CHT-OP-TU (UO 2 powder and pellets),

3) VersaPac (U-metal/TRISO),

4) TN-B1 (BWR fuel assemblies), and

5) $\mathrm{DN}-30\left(\mathrm{UF}_{6}\right)$

The results provided for each package evaluation include enrichment and packaging limits (e.g., maximum HAC package array size as a function of enrichment) and the identification of benchmark critical experiments that are appropriate candidates for use in computer code validation (determination of $k_{\text {eff }}$ bias and bias uncertainty for the application).

Table 31 summarizes the range of enrichment and transportation array size combinations that each design could support based on criticality calculations. In some cases, additional trade-off options to permit increased enrichment are indicated (i.e., increased Gd rod credit in BWR assemblies). These criticality analysis results indicate that there are viable means for increasing enrichments into the HALEU range across the spectrum of fuel forms with differing increase amounts available for different packages.

The Traveller package can support transportation of PWR and $\mathrm{BWR} \mathrm{UO}_{2}$ fuel rods up to $10 \mathrm{wt} \%$ in the rod pipe container. Determining an upper fuel assembly enrichment limit for the Traveller is difficult due to the complexity of the safety analysis, which covers numerous fuel designs and three versions of the package. Subcritical margin is relatively insensitive to package array size due to the use of Boral plates and polyethylene moderator blocks, which limit neutronic interaction between packages. The Traveller standard version could support transportation of 5.5 to $6.5 \mathrm{wt} \%{ }^{235} \mathrm{U}$ (assembly average) PWR fuel assemblies for some fuel designs through a combination of transportation array size limits and safety analysis margin harvesting. In addition, maximum enrichment can be increased by crediting minimum integral poisons in the fuel assembly, similar to the TN-B1 BWR assembly package. Numerous critical experiment candidates are available for validation of 5 to $8 \mathrm{wt} \%{ }^{235} \mathrm{U}$ Traveller models.

The CHT-OP-TU package can be configured to transport up to $18 \mathrm{wt} \% \mathrm{UO}_{2}$ powder and up to $16.5 \mathrm{wt} \%$ $\mathrm{UO}_{2}$ pellets through a combination of oxide vessel diameter and package array size. Numerous critical experiment candidates are available for validation of 5 to $16.5 \mathrm{wt} \%{ }^{235} \mathrm{U}$ CHT-OP-TU models.

The Versa-Pac package is currently licensed to transport $U$ materials enriched up to $100 \%{ }^{235} \mathrm{U}$ with a ${ }^{235} \mathrm{U}$ content maximum allowed mass determined by enrichment bin and packaging type. Because the Versa-Pac is already licensed to transport HALEU and has mass limits that are a function of enrichment, no additional evaluation was performed.

The TN-B1 package can support transportation of BWR fuel assemblies up to $10 \mathrm{wt} \%$ (assembly average) using a combination of package array size and gadolinia rod credit. Subcritical margin is highly sensitive to package array size in the un-poisoned TN-B1. Numerous critical experiment candidates were identified for validation of 5 to $8 \mathrm{wt} \%{ }^{235} \mathrm{U}$ TN-B1 models.

The DN-30 package can support transportation of $\mathrm{UF}_{6}$ up to $9.5 \mathrm{wt} \%$ by reducing transportation array size from unlimited $\left(5 \mathrm{wt} \%{ }^{235} \mathrm{U}\right)$ to 2 packages $\left(9.5 \mathrm{wt} \%{ }^{235} \mathrm{U}\right)$. These results are based on retaining the 10 
CFR 71.55(b) exemption from the assumption of water leakage into the containment system. Because the limiting accident conditions do not include water in-leakage, the neutron energy spectrum of the DN-30 is harder than other evaluated packages and only a few critical experiments were identified with a similarity index $\left(\mathrm{c}_{\mathrm{k}}\right)$ of 0.8 or higher. Discussion of methods for validation penalty assessment is provided in the DN-30 analysis.

Table 31. Package array criticality summary.

\begin{tabular}{|c|c|c|c|c|}
\hline Package & Fuel form & $\begin{array}{l}\text { Enrichment } \\
\left(\text { wt. } \%{ }^{235} U\right)\end{array}$ & $\begin{array}{l}\text { HAC array } \\
\text { size }\end{array}$ & Notes \\
\hline Traveller & PWR FA $^{1}$ & 5 & Varies & Current limit. \\
\hline Traveller & PWR FA $^{1}$ & $\sim 6$ & Small & $\begin{array}{l}\text { Enrichment increases } \sim 0.5 \% \text { with array size } \\
\text { halved. Additional margin is available for } \\
\text { some fuel designs and package versions. }\end{array}$ \\
\hline Traveller & PWR FA $^{1}$ & $\sim 7$ & 1 & $\begin{array}{l}\text { Approximate limit for a single package } \\
\text { without additional margin credit. }\end{array}$ \\
\hline Traveller & PWR FA $^{1}$ & 8 & Large & $\begin{array}{l}\text { Same array size as for the current limit with } \\
\text { credit for } 52 \text { IFBA per fuel assembly. }\end{array}$ \\
\hline Traveller & $\begin{array}{l}\text { PWR/BWR fuel } \\
\text { pins }\end{array}$ & $>10$ & Infinite & $\begin{array}{l}\text { Additional margin is available to support } \\
\text { higher enrichment. }\end{array}$ \\
\hline CHT-OP-TU & $\mathrm{UO}_{2}$ powder & 5 & 50 & Current limit, 8 in. pipe. \\
\hline CHT-OP-TU & $\mathrm{UO}_{2}$ powder & 8 & 18 & 7.5 in. pipe. \\
\hline CHT-OP-TU & $\mathrm{UO}_{2}$ powder & 18 & 48 & 6 in. pipe. \\
\hline CHT-OP-TU & $\mathrm{UO}_{2}$ pellets & 5 & 50 & Current limit, 7.5 in. pipe. \\
\hline CHT-OP-TU & $\mathrm{UO}_{2}$ pellets & 6.9 & 18 & 7.5 in. pipe. \\
\hline CHT-OP-TU & $\mathrm{UO}_{2}$ pellets & 16.5 & 48 & 6 in. pipe. \\
\hline Versa-Pac & Multiple & 10 & 100 & 55 gal drum, 5 in. pipe, $1,605 \mathrm{~g}^{235} \mathrm{U}$. \\
\hline Versa-Pac & Multiple & 20 & 100 & 55 gal drum, 5 in. pipe, $1,215 \mathrm{~g}{ }^{235} \mathrm{U}$. \\
\hline TN-B1 & BWR FA $^{2}$ & 5 & 100 & Current limit, $13 \mathrm{Gd}$ rods/assembly. \\
\hline TN-B1 & $\mathrm{BWR}_{\mathrm{FA}}{ }^{2}$ & 6 & 49 & $13 \mathrm{Gd}$ rods/assembly. \\
\hline TN-B1 & BWR FA $^{2}$ & 7.8 & 25 & $13 \mathrm{Gd}$ rods/assembly. \\
\hline TN-B1 & BWR FA $^{2}$ & 9.8 & 16 & $13 \mathrm{Gd}$ rods/assembly. \\
\hline TN-B1 & $\mathrm{BWR}^{\mathrm{FA}}{ }^{2}$ & 8 & 100 & $24 \mathrm{Gd}$ rods/assembly. \\
\hline $\mathrm{DN}-30$ & $\mathrm{UF}_{6}$ & 5 & Infinite & Current limit. Few benchmark candidates. \\
\hline DN-30 & $\mathrm{UF}_{6}$ & 6.7 & 6 & HUR sphere governs. \\
\hline $\mathrm{DN}-30$ & $\mathrm{UF}_{6}$ & 12.5 & 1 & $\begin{array}{l}\text { HUR sphere governs. Few benchmark } \\
\text { candidates. }\end{array}$ \\
\hline
\end{tabular}

${ }^{1}$ PWR FA = PWR fuel assembly, one per package, assembly average enrichment

${ }^{2} \mathrm{BWR} F A=\mathrm{BWR}$ fuel assemblies, two per package, assembly average enrichment

Appendix A summarizes the results of similarity calculations for representative cases from four of the five package designs; the Versa-Pac is already licensed for enrichments up to $100 \%$. Color-coding identifies critical experiments that are similar (green, $c_{k} \geq 0.9$ ) and that are marginally similar (yellow, $c_{k} \geq 0.8$ ). These experiments are considered valid candidates for criticality code validation for the analyzed packages at limiting conditions, subject to further screening based on the appropriateness of materials and geometry. Numerous experiments are indicted by $c_{k}$ to be generally applicable across the range of analyzed packages.

Appendix B addresses the effect of using multiple vs. single water compositions on $c_{k}$. Appendix C investigates the effect of array size on benchmark experiment similarity. 


\section{ACKNOWLEDGMENTS}

Support for this work was provided by the NRC Office of Nuclear Regulatory Research and the Office of Nuclear Material Safety and Safeguards. The authors would like to thank many ORNL staff members for their feedback on the contents and presentation in this report. 


\section{REFERENCES}

[1] M. Diaz, 2019, DSFM Regulatory Conference, "Advanced Fuels Update on the Front End of the Fuel Cycle,” https://www.nrc.gov/docs/ML1925/ML19255F598.pdf.

[2] F. Pimental et al., "The Economic Benefits and Challenges with Utilizing Increased Enrichment and Fuel Burnup for Light-Water Reactors," Nuclear Energy Institute (2019).

[3] B. T. Rearden and M. A. Jessee, SCALE Code System, ORNL/TM-2005/39, Version 6.2.3, Oak Ridge National Laboratory, Oak Ridge, Tennessee (2018).

[4] B. T. Rearden et al., "Sensitivity and Uncertainty Analysis Capabilities and Data in SCALE," Nucl. Technol. 174, 236-288 (2011).

[5] Westinghouse Electric Company, "Application for Certificate of Compliance for the Traveller PWR Fuel Shipping Package,” NRC ADAMS ML 19098A933 (Safety Analysis Report Revision 14) (2019).

[6] US NRC, Certificate of Compliance 9297, Revision 11 (2019).

[7] Columbiana Hi Tech, LLC, Safety Analysis Report for the Model CHT OP-TU, Revision 7 (2006).

[8] US NRC, Certificate of Compliance 9288, Revision 11 (2018).

[9] Daher-TLI, Versa-Pac Safety Analysis Report, Rev. 10, ADAMS Accession number ML18087A454 (2018).

[10] US NRC, Certificate of Compliance 9342, Revision 15 (2020).

[11] Framatome, Inc., Framatome TN-B1 Safety Analysis Report, Rev. 9, ADAMS Accession number ML19045A485 (2019).

[12] US NRC, Certificate of Compliance 9372, Revision 2 (2019).

[13] Daher-TLI, Safety Analysis Report of the DN30 Package, Rev. 3, ADAMS Accession number ML19200A133 (2019).

[14] US NRC, Certificate of Compliance 9362, Revision 2 (2019).

[15] J. M. Scaglione, D. E. Mueller, J. C. Wagner, and W. J. Marshall, An Approach for Validating Actinide and Fission Product Burnup Credit Criticality Safety Analyses-Criticality (keff) Predictions, NUREG/CR-7109 (ORNL/TM-2011/514), Oak Ridge National Laboratory, Oak Ridge, Tennessee (2012).

[16] B. L. Broadhead et al., "Sensitivity- and Uncertainty-Based Criticality Safety Validation Techniques," Nucl. Sci. Eng. 146, 340-366 (2004).

[17] International Criticality Safety Benchmark Evaluation Project ICSBEP Handbook, NEA/NSC/DOC(95)03, Organisation for Economic Co-Operation and Development /Nuclear Energy Agency (2019).

[18] W. J. Marshall and B. T. Rearden, "The SCALE Verified, Archived Library of Inputs and Data VALID," ANS Nuclear Criticality Safety Division Topical Meetings (NCSD2013), Wilmington, North Carolina (2013).

[19] J. B. Clarity, W. J. Marshall, and E. M. Saylor, "User Experiences with ICSBEP Distributed Sensitivity Data Profiles with the SCALE Sensitivity and Uncertainty Methods as of Winter 2019," Data, Analysis and Operations in Nuclear Criticality Safety-III (2019).

[20] 10 CFR 71, NRC Regulations, Packaging and Transportation of Radioactive Material. 
[21] W.C. Jordan J.C. Turner, Estimated Critical Conditions for $\mathrm{UO}_{2} \mathrm{~F}_{2}-\mathrm{H}_{2} \mathrm{O}$ Systems in Fully WaterReflected Spherical Geometry, ORNL/TM-12292 (1992)

[22] L. Begue et al., "Criticality Safety of Enriched UF6 Cylinders." Proceedings of the 17th International Symposium on the Packaging and Transportation of Radioactive Materials (2013).

[23] J. M. Scaglione, D. E. Mueller, J. C. Wagner, and W. J. Marshall, An Approach for Validating Actinide and Fission Product Burnup Credit Criticality Safety Analyses-Criticality (keff) Predictions, NUREG/CR 7109 (ORNL/TM-2011/514), Oak Ridge, Tennessee (2012).

[24] W. J. Marshall, B. J. Ade, S. M. Bowman, I. C. Gauld, G. Ilas, U. Mertyurek, and G. Radulescu, Technical Basis for Peak Reactivity Burnup Credit for BWR Spent Nuclear Fuel in Storage and Transportation Systems, NUREG/CR-7194 (ORNL/TM-2014/240), Oak Ridge, Tennessee (2015).

[25] W. J. Marshall, J. B. Clarity, and S. M. Bowman, Validation of keff Calculations for Extended BWR Burnup Credit, NUREG/CR-7252 (ORNL/TM-2018/797), Oak Ridge, Tennessee (2018).

[26] B. T. Rearden, M. L. Williams, M. A. Jessee, D. E. Mueller, and D. A. Wiarda, "Sensitivity and Uncertainty Analysis Capabilities and Data in SCALE," Nucl. Technol. 174, 236-288 (2011).

[27] “DN30 Package,” Daher Nuclear Technologies; https://daher-nuclear-technologies.com/en/dn30package (current as of Nov. 4, 2020). 


\section{APPENDIX A. BENCHMARK EXPERIMENT SIMILARITY TABLE}

Appendix A summarizes the results of similarity calculations for representative cases from four of the five package designs. Table A-1 contains the column code index descriptions. Cells highlighted yellow have $c_{k} \geq 0.8$. Cells highlighted green have $c_{k} \geq 0.9$.

Table A-1. Similarity table package number description.

\begin{tabular}{|c|c|c|c|c|}
\hline Package \# & Package description & $\begin{array}{c}\text { Enrichment } \\
(\mathrm{wt} \% \text { 235U) }\end{array}$ & \# Packages & Type \\
\hline 1 & Traveller fuel assembly $(17 \mathrm{x} 17)$ & 5 & Infinite & PWR FA \\
\hline 2 & Traveller fuel assembly & 8 & 1 & PWR FA \\
\hline 3 & Traveller fuel assembly, 54 IFBA / FA & 8 & Infinite & PWR FA \\
\hline 4 & Traveller rod pipe & 5 & Infinite & Pins \\
\hline 5 & Traveller rod pipe & 10 & Infinite & Pins \\
\hline 6 & CHT-OP-TU UO2 powder & 5 & 48 & Powder \\
\hline 7 & CHT-OP-TU UO2 powder & 8 & 18 & Powder \\
\hline 8 & CHT-OP-TU UO2 pellets & 6.9 & 18 & Pellets \\
\hline 9 & CHT-OP-TU UO2 pellets & 16.5 & 48 & Pellets \\
\hline 10 & TN-B1 BWR FA (11x11, 13 Gd rods/FA) & 5 & 100 & BWR FA \\
\hline 11 & TN-B1 BWR FA (11x11, 13 Gd rods/FA) & 6.7 & 36 & BWR FA \\
\hline 12 & TN-B1 BWR FA (11x11, 24 Gd rods/FA) & 8 & 100 & BWR FA \\
\hline 13 & DX-30 UF6 in 30B (mod exclusion) & 5 & Infinite & UF6 \\
\hline 14 & DX-30 UF6 in 30B (mod exclusion) & 6.7 & 6 & UF6 \\
\hline 15 & DX-30 UF6 in 30B (optimum mod) & 12.5 & 1 & UF6 \\
\hline
\end{tabular}




\begin{tabular}{|c|c|c|c|c|c|c|c|c|c|c|c|c|c|c|c|c|}
\hline Case & Experiment & 1 & 2 & 3 & 4 & 5 & 6 & 7 & 8 & 9 & 10 & 11 & 12 & 13 & 14 & 15 \\
\hline 1 & IEU-COMP-FAST-004-001_E & -0.14 & -0.18 & -0.09 & -0.20 & -0.17 & -0.10 & -0.12 & -0.12 & -0.07 & 0.00 & -0.04 & 0.05 & 0.49 & 0.34 & 0.51 \\
\hline 2 & IEU-COMP-INTER-005-001_ & -0.02 & -0.05 & 0.02 & -0.08 & -0.05 & -0.01 & -0.01 & 0.00 & 0.03 & 0.07 & 0.05 & 0.12 & 0.55 & 0.44 & 0.62 \\
\hline 3 & IEU-COMP-INTER-006-001_ & 0.26 & 0.29 & 0.26 & 0.25 & 0.24 & 0.20 & 0.23 & 0.23 & 0.22 & 0.13 & 0.17 & 0.12 & 0.09 & 0.11 & 0.11 \\
\hline 4 & IEU-COMP-MIXED-001-001_ & 0.68 & 0.72 & 0.71 & 0.68 & 0.71 & 0.60 & 0.66 & 0.66 & 0.65 & 0.54 & 0.64 & 0.58 & 0.57 & 0.82 & 0.91 \\
\hline 5 & IEU-COMP-MIXED-001-017_ & 0.71 & 0.76 & 0.74 & 0.73 & 0.75 & 0.63 & 0.70 & 0.70 & 0.68 & 0.54 & 0.65 & 0.57 & 0.50 & 0.79 & 0.86 \\
\hline 6 & IEU-COMP-MIXED-001-021_ & 0.68 & 0.72 & 0.72 & 0.69 & 0.71 & 0.60 & 0.66 & 0.67 & 0.66 & 0.54 & 0.64 & 0.58 & 0.57 & 0.81 & 0.91 \\
\hline 7 & IEU-COMP-MIXED-001-022_- & 0.73 & 0.77 & 0.75 & 0.75 & 0.77 & 0.65 & 0.71 & 0.71 & 0.70 & 0.56 & 0.67 & 0.59 & 0.51 & 0.80 & 0.86 \\
\hline 8 & IEU-COMP-MIXED-004-004 & 0.27 & 0.28 & 0.25 & 0.27 & 0.26 & 0.23 & 0.23 & 0.24 & 0.22 & 0.18 & 0.20 & 0.16 & 0.10 & 0.12 & 0.11 \\
\hline 9 & IEU-COMP-MIXED-004-005_ & 0.27 & 0.27 & 0.25 & 0.27 & 0.26 & 0.23 & 0.23 & 0.24 & 0.22 & 0.18 & 0.20 & 0.16 & 0.10 & 0.12 & 0.11 \\
\hline 10 & IEU-COMP-THERM-001-002_ & 0.76 & 0.82 & 0.79 & 0.80 & 0.83 & 0.70 & 0.77 & 0.76 & 0.76 & 0.59 & 0.71 & 0.62 & 0.47 & 0.74 & 0.79 \\
\hline 11 & IEU-COMP-THERM-001-003_ & 0.77 & 0.84 & 0.79 & 0.84 & 0.87 & 0.72 & 0.81 & 0.77 & 0.79 & 0.60 & 0.72 & 0.62 & 0.38 & 0.64 & 0.64 \\
\hline 12 & IEU-COMP-THERM-001-004_- & 0.75 & 0.83 & 0.76 & 0.83 & 0.86 & 0.72 & 0.80 & 0.76 & 0.79 & 0.59 & 0.71 & 0.61 & 0.33 & 0.56 & 0.55 \\
\hline 13 & IEU-COMP-THERM-001-005_- & 0.69 & 0.72 & 0.70 & 0.72 & 0.75 & 0.69 & 0.74 & 0.70 & 0.72 & 0.63 & 0.71 & 0.64 & 0.34 & 0.49 & 0.43 \\
\hline 14 & IEU-COMP-THERM-001-006_ & 0.77 & 0.84 & 0.79 & 0.84 & 0.87 & 0.72 & 0.80 & 0.77 & 0.79 & 0.60 & 0.72 & 0.62 & 0.38 & 0.64 & 0.65 \\
\hline 15 & IEU-COMP-THERM-001-007_- & 0.77 & 0.84 & 0.79 & 0.84 & 0.87 & 0.72 & 0.80 & 0.77 & 0.79 & 0.60 & 0.73 & 0.63 & 0.39 & 0.65 & 0.66 \\
\hline 16 & IEU-COMP-THERM-001-008_ & 0.78 & 0.84 & 0.79 & 0.83 & 0.86 & 0.72 & 0.80 & 0.78 & 0.79 & 0.61 & 0.73 & 0.63 & 0.40 & 0.66 & 0.67 \\
\hline 17 & IEU-COMP-THERM-001-009_- & 0.76 & 0.81 & 0.78 & 0.79 & 0.82 & 0.69 & 0.76 & 0.75 & 0.75 & 0.60 & 0.71 & 0.63 & 0.48 & 0.75 & 0.80 \\
\hline 18 & IEU-COMP-THERM-001-010_- & 0.77 & 0.82 & 0.79 & 0.80 & 0.83 & 0.70 & 0.77 & 0.76 & 0.76 & 0.60 & 0.72 & 0.63 & 0.48 & 0.75 & 0.79 \\
\hline 19 & IEU-COMP-THERM-001-011_ & 0.77 & 0.83 & 0.79 & 0.81 & 0.83 & 0.70 & 0.78 & 0.76 & 0.76 & 0.60 & 0.72 & 0.63 & 0.48 & 0.74 & 0.79 \\
\hline 20 & IEU-COMP-THERM-001-012_ & 0.77 & 0.83 & 0.80 & 0.81 & 0.84 & 0.71 & 0.78 & 0.76 & 0.77 & 0.61 & 0.73 & 0.64 & 0.48 & 0.74 & 0.78 \\
\hline 21 & IEU-COMP-THERM-001-013 & 0.75 & 0.83 & 0.76 & 0.83 & 0.86 & 0.72 & 0.80 & 0.76 & 0.79 & 0.59 & 0.71 & 0.61 & 0.33 & 0.56 & 0.55 \\
\hline 22 & IEU-COMP-THERM-001-014_- & 0.75 & 0.83 & 0.76 & 0.83 & 0.86 & 0.72 & 0.80 & 0.76 & 0.79 & 0.59 & 0.71 & 0.61 & 0.33 & 0.56 & 0.54 \\
\hline 23 & IEU-COMP-THERM-001-015_- & 0.75 & 0.83 & 0.77 & 0.83 & 0.86 & 0.73 & 0.80 & 0.76 & 0.79 & 0.60 & 0.72 & 0.62 & 0.34 & 0.56 & 0.54 \\
\hline 24 & IEU-COMP-THERM-001-016_ & 0.73 & 0.79 & 0.74 & 0.79 & 0.83 & 0.72 & 0.78 & 0.74 & 0.77 & 0.61 & 0.71 & 0.62 & 0.32 & 0.52 & 0.48 \\
\hline 25 & IEU-COMP-THERM-001-018_ & 0.76 & 0.83 & 0.78 & 0.82 & 0.85 & 0.70 & 0.78 & 0.76 & 0.77 & 0.57 & 0.70 & 0.60 & 0.40 & 0.69 & 0.71 \\
\hline 26 & IEU-COMP-THERM-001-019 & 0.74 & 0.80 & 0.75 & 0.81 & 0.84 & 0.72 & 0.79 & 0.75 & 0.78 & 0.60 & 0.71 & 0.62 & 0.33 & 0.54 & 0.51 \\
\hline 27 & IEU-COMP-THERM-001-020_- & 0.76 & 0.82 & 0.79 & 0.80 & 0.83 & 0.70 & 0.77 & 0.76 & 0.76 & 0.60 & 0.72 & 0.63 & 0.48 & 0.75 & 0.79 \\
\hline 28 & IEU-COMP-THERM-001-023_ & 0.77 & 0.84 & 0.79 & 0.83 & 0.85 & 0.71 & 0.79 & 0.77 & 0.78 & 0.59 & 0.72 & 0.62 & 0.42 & 0.69 & 0.71 \\
\hline 29 & IEU-COMP-THERM-001-024_ & 0.77 & 0.83 & 0.78 & 0.82 & 0.85 & 0.71 & 0.79 & 0.76 & 0.77 & 0.59 & 0.71 & 0.62 & 0.43 & 0.70 & 0.72 \\
\hline 30 & IEU-COMP-THERM-001-025_- & 0.74 & 0.80 & 0.75 & 0.80 & 0.84 & 0.73 & 0.80 & 0.75 & 0.78 & 0.62 & 0.73 & 0.63 & 0.34 & 0.54 & 0.51 \\
\hline 31 & IEU-COMP-THERM-001-026_- & 0.73 & 0.79 & 0.74 & 0.79 & 0.83 & 0.72 & 0.79 & 0.74 & 0.77 & 0.62 & 0.72 & 0.63 & 0.33 & 0.53 & 0.49 \\
\hline 32 & IEU-COMP-THERM-001-027_- & 0.73 & 0.79 & 0.74 & 0.79 & 0.83 & 0.72 & 0.79 & 0.74 & 0.77 & 0.62 & 0.72 & 0.63 & 0.33 & 0.53 & 0.49 \\
\hline 33 & IEU-COMP-THERM-001-028_ & 0.77 & 0.82 & 0.79 & 0.80 & 0.83 & 0.70 & 0.77 & 0.76 & 0.76 & 0.60 & 0.72 & 0.63 & 0.49 & 0.75 & 0.80 \\
\hline 34 & IEU-COMP-THERM-001-029 & 0.77 & 0.83 & 0.80 & 0.81 & 0.83 & 0.70 & 0.78 & 0.76 & 0.76 & 0.61 & 0.72 & 0.64 & 0.48 & 0.75 & 0.79 \\
\hline 35 & IEU-COMP-THERM-015-002_- & 0.23 & 0.26 & 0.22 & 0.24 & 0.24 & 0.19 & 0.21 & 0.21 & 0.20 & 0.12 & 0.15 & 0.11 & 0.09 & 0.12 & 0.11 \\
\hline 36 & IEU-COMP-THERM-015-003_- & 0.22 & 0.24 & 0.21 & 0.24 & 0.23 & 0.18 & 0.21 & 0.21 & 0.19 & 0.12 & 0.15 & 0.11 & 0.09 & 0.12 & 0.10 \\
\hline 37 & IEU-COMP-THERM-015-006_- & 0.25 & 0.26 & 0.23 & 0.26 & 0.25 & 0.21 & 0.22 & 0.23 & 0.21 & 0.16 & 0.18 & 0.14 & 0.10 & 0.12 & 0.11 \\
\hline 38 & IEU-COMP-THERM-015-007_- & 0.25 & 0.26 & 0.23 & 0.26 & 0.25 & 0.22 & 0.22 & 0.23 & 0.21 & 0.17 & 0.19 & 0.15 & 0.10 & 0.12 & 0.11 \\
\hline 39 & IEU-COMP-THERM-015-008_ & 0.23 & 0.24 & 0.21 & 0.25 & 0.24 & 0.20 & 0.21 & 0.21 & 0.20 & 0.16 & 0.17 & 0.13 & 0.10 & 0.12 & 0.10 \\
\hline 40 & IEU-COMP-THERM-015-009_- & 0.23 & 0.24 & 0.21 & 0.25 & 0.24 & 0.20 & 0.21 & 0.21 & 0.20 & 0.15 & 0.17 & 0.13 & 0.10 & 0.12 & 0.10 \\
\hline 41 & IEU-COMP-THERM-015-010_- & 0.23 & 0.23 & 0.21 & 0.25 & 0.24 & 0.20 & 0.21 & 0.21 & 0.20 & 0.16 & 0.17 & 0.13 & 0.10 & 0.12 & 0.10 \\
\hline 42 & IEU-COMP-THERM-015-011_- & 0.22 & 0.22 & 0.20 & 0.25 & 0.24 & 0.20 & 0.20 & 0.20 & 0.19 & 0.15 & 0.17 & 0.13 & 0.10 & 0.12 & 0.10 \\
\hline
\end{tabular}




\begin{tabular}{|c|c|c|c|c|c|c|c|c|c|c|c|c|c|c|c|c|}
\hline Case & Experiment & 1 & 2 & 3 & 4 & 5 & 6 & 7 & 8 & 9 & 10 & 11 & 12 & 13 & 14 & 15 \\
\hline 43 & IEU-COMP-THERM-015-012 & 0.22 & 0.23 & 0.20 & 0.25 & 0.24 & 0.20 & 0.20 & 0.20 & 0.19 & 0.15 & 0.17 & 0.13 & 0.10 & 0.12 & 0.10 \\
\hline 44 & IEU-COMP-THERM-015-013_ & 0.22 & 0.23 & 0.20 & 0.25 & 0.24 & 0.20 & 0.20 & 0.20 & 0.19 & 0.15 & 0.17 & 0.13 & 0.10 & 0.12 & 0.10 \\
\hline 45 & IEU-COMP-THERM-015-014_ & 0.22 & 0.22 & 0.20 & 0.25 & 0.24 & 0.20 & 0.20 & 0.20 & 0.19 & 0.16 & 0.17 & 0.13 & 0.10 & 0.12 & 0.10 \\
\hline 46 & IEU-COMP-THERM-015-015_ & 0.22 & 0.22 & 0.20 & 0.25 & 0.24 & 0.20 & 0.20 & 0.20 & 0.19 & 0.16 & 0.17 & 0.13 & 0.10 & 0.12 & 0.10 \\
\hline 47 & IEU-COMP-THERM-015-016_ & 0.21 & 0.21 & 0.19 & 0.24 & 0.23 & 0.19 & 0.19 & 0.19 & 0.18 & 0.15 & 0.16 & 0.13 & 0.09 & 0.11 & 0.10 \\
\hline 48 & IEU-COMP-THERM-015-017_ & 0.21 & 0.21 & 0.19 & 0.24 & 0.23 & 0.19 & 0.19 & 0.19 & 0.18 & 0.15 & 0.16 & 0.13 & 0.09 & 0.11 & 0.10 \\
\hline 49 & IEU-COMP-THERM-015-018_ & 0.21 & 0.21 & 0.19 & 0.24 & 0.23 & 0.19 & 0.19 & 0.19 & 0.18 & 0.15 & 0.16 & 0.13 & 0.09 & 0.11 & 0.10 \\
\hline 50 & IEU-COMP-THERM-016-001_- & 0.23 & 0.24 & 0.22 & 0.25 & 0.24 & 0.20 & 0.21 & 0.21 & 0.20 & 0.15 & 0.17 & 0.13 & 0.10 & 0.12 & 0.10 \\
\hline 51 & IEU-COMP-THERM-016-002_ & 0.22 & 0.23 & 0.20 & 0.25 & 0.24 & 0.20 & 0.20 & 0.21 & 0.19 & 0.15 & 0.16 & 0.13 & 0.10 & 0.12 & 0.10 \\
\hline 52 & IEU-COMP-THERM-016-003_ & 0.22 & 0.23 & 0.21 & 0.25 & 0.24 & 0.20 & 0.20 & 0.21 & 0.19 & 0.15 & 0.16 & 0.13 & 0.10 & 0.12 & 0.10 \\
\hline 53 & IEU-COMP-THERM-016-004_ & 0.22 & 0.23 & 0.21 & 0.25 & 0.24 & 0.20 & 0.20 & 0.21 & 0.19 & 0.15 & 0.17 & 0.13 & 0.10 & 0.12 & 0.10 \\
\hline 54 & IEU-COMP-THERM-016-005_- & 0.21 & 0.22 & 0.19 & 0.24 & 0.23 & 0.19 & 0.19 & 0.19 & 0.18 & 0.14 & 0.16 & 0.12 & 0.09 & 0.11 & 0.10 \\
\hline 55 & IEU-COMP-THERM-016-011_- & 0.23 & 0.24 & 0.22 & 0.25 & 0.24 & 0.20 & 0.21 & 0.21 & 0.20 & 0.15 & 0.17 & 0.13 & 0.10 & 0.12 & 0.10 \\
\hline 56 & IEU-COMP-THERM-016-012_ & 0.23 & 0.24 & 0.21 & 0.25 & 0.24 & 0.20 & 0.21 & 0.21 & 0.20 & 0.15 & 0.17 & 0.13 & 0.10 & 0.12 & 0.10 \\
\hline 57 & IEU-COMP-THERM-016-013_ & 0.23 & 0.24 & 0.21 & 0.25 & 0.24 & 0.20 & 0.21 & 0.21 & 0.20 & 0.15 & 0.17 & 0.13 & 0.10 & 0.12 & 0.10 \\
\hline 58 & IEU-COMP-THERM-016-014_ & 0.23 & 0.24 & 0.21 & 0.25 & 0.24 & 0.20 & 0.21 & 0.21 & 0.20 & 0.15 & 0.17 & 0.13 & 0.10 & 0.12 & 0.10 \\
\hline 59 & IEU-COMP-THERM-016-015_- & 0.22 & 0.23 & 0.20 & 0.25 & 0.24 & 0.20 & 0.20 & 0.20 & 0.19 & 0.15 & 0.17 & 0.13 & 0.10 & 0.12 & 0.10 \\
\hline 60 & IEU-COMP-THERM-016-016_ & 0.22 & 0.23 & 0.20 & 0.25 & 0.24 & 0.20 & 0.20 & 0.20 & 0.19 & 0.15 & 0.17 & 0.13 & 0.10 & 0.12 & 0.10 \\
\hline 61 & IEU-COMP-THERM-016-017_ & 0.22 & 0.23 & 0.20 & 0.25 & 0.24 & 0.20 & 0.20 & 0.20 & 0.19 & 0.15 & 0.17 & 0.13 & 0.10 & 0.12 & 0.10 \\
\hline 62 & IEU-COMP-THERM-016-018_- & 0.22 & 0.23 & 0.20 & 0.25 & 0.24 & 0.20 & 0.20 & 0.20 & 0.19 & 0.15 & 0.17 & 0.13 & 0.10 & 0.12 & 0.10 \\
\hline 63 & IEU-COMP-THERM-016-019_ & 0.20 & 0.21 & 0.19 & 0.24 & 0.23 & 0.19 & 0.19 & 0.19 & 0.18 & 0.15 & 0.16 & 0.12 & 0.10 & 0.12 & 0.10 \\
\hline 64 & IEU-COMP-THERM-016-020_ & 0.20 & 0.21 & 0.19 & 0.24 & 0.23 & 0.19 & 0.19 & 0.19 & 0.18 & 0.14 & 0.16 & 0.12 & 0.10 & 0.12 & 0.10 \\
\hline 65 & IEU-COMP-THERM-016-021_ & 0.23 & 0.23 & 0.21 & 0.25 & 0.24 & 0.20 & 0.21 & 0.21 & 0.19 & 0.15 & 0.17 & 0.13 & 0.10 & 0.12 & 0.10 \\
\hline 66 & IEU-COMP-THERM-016-022_ & 0.23 & 0.23 & 0.21 & 0.25 & 0.24 & 0.20 & 0.21 & 0.21 & 0.19 & 0.16 & 0.17 & 0.14 & 0.10 & 0.12 & 0.10 \\
\hline 67 & IEU-COMP-THERM-016-024_- & 0.23 & 0.24 & 0.22 & 0.25 & 0.24 & 0.20 & 0.21 & 0.21 & 0.20 & 0.15 & 0.17 & 0.13 & 0.10 & 0.12 & 0.10 \\
\hline 68 & IEU-COMP-THERM-016-025_ & 0.22 & 0.23 & 0.20 & 0.25 & 0.24 & 0.20 & 0.20 & 0.20 & 0.19 & 0.15 & 0.17 & 0.13 & 0.10 & 0.12 & 0.10 \\
\hline 69 & IEU-COMP-THERM-016-026_- & 0.00 & 0.00 & 0.00 & 0.04 & 0.04 & 0.00 & 0.00 & 0.00 & 0.00 & 0.01 & 0.01 & 0.01 & 0.00 & 0.00 & 0.00 \\
\hline 70 & IEU-COMP-THERM-016-027_ & 0.00 & 0.00 & 0.00 & 0.05 & 0.05 & 0.02 & 0.02 & 0.02 & 0.03 & 0.03 & 0.03 & 0.03 & 0.02 & 0.01 & 0.01 \\
\hline 71 & IEU-COMP-THERM-016-028_ & 0.00 & 0.00 & 0.00 & 0.05 & 0.05 & 0.01 & 0.01 & 0.01 & 0.02 & 0.02 & 0.02 & 0.02 & 0.01 & 0.01 & 0.00 \\
\hline 72 & IEU-COMP-THERM-016-029_- & 0.21 & 0.21 & 0.19 & 0.24 & 0.23 & 0.19 & 0.19 & 0.19 & 0.18 & 0.14 & 0.16 & 0.12 & 0.10 & 0.12 & 0.10 \\
\hline 73 & IEU-COMP-THERM-016-031_- & 0.00 & 0.00 & 0.00 & 0.04 & 0.04 & 0.00 & 0.00 & 0.00 & 0.00 & 0.01 & 0.01 & 0.01 & 0.00 & 0.00 & 0.00 \\
\hline 74 & IEU-COMP-THERM-016-032_- & 0.00 & 0.00 & 0.00 & 0.04 & 0.04 & 0.00 & 0.00 & 0.00 & 0.00 & 0.01 & 0.01 & 0.01 & 0.00 & 0.00 & 0.00 \\
\hline 75 & IEU-COMP-THERM-016-033_ & 0.00 & 0.00 & 0.00 & 0.04 & 0.04 & 0.00 & 0.00 & 0.00 & 0.00 & 0.01 & 0.01 & 0.01 & 0.00 & 0.00 & 0.00 \\
\hline 76 & IEU-COMP-THERM-016-034_- & 0.00 & 0.00 & 0.00 & 0.04 & 0.04 & 0.00 & 0.00 & 0.00 & 0.00 & 0.01 & 0.01 & 0.01 & 0.00 & 0.00 & 0.00 \\
\hline 77 & IEU-COMP-THERM-016-035_ & 0.00 & 0.00 & 0.00 & 0.04 & 0.04 & 0.00 & 0.00 & 0.00 & 0.00 & 0.01 & 0.01 & 0.01 & 0.00 & 0.00 & 0.00 \\
\hline 78 & IEU-COMP-THERM-016-036_ & 0.00 & 0.00 & 0.00 & 0.04 & 0.04 & 0.00 & 0.00 & 0.00 & 0.00 & 0.01 & 0.01 & 0.01 & 0.00 & 0.00 & 0.00 \\
\hline 79 & IEU-COMP-THERM-016-037_- & 0.00 & 0.00 & 0.00 & 0.04 & 0.04 & 0.00 & 0.00 & 0.00 & 0.00 & 0.01 & 0.01 & 0.01 & 0.00 & 0.00 & 0.00 \\
\hline 80 & IEU-COMP-THERM-016-038_ & 0.00 & 0.00 & 0.00 & 0.04 & 0.04 & 0.00 & 0.00 & 0.00 & 0.00 & 0.01 & 0.01 & 0.01 & 0.00 & 0.00 & 0.00 \\
\hline 81 & IEU-COMP-THERM-016-039 & 0.00 & 0.00 & 0.00 & 0.04 & 0.04 & 0.00 & 0.00 & 0.00 & 0.00 & 0.01 & 0.01 & 0.01 & 0.00 & 0.00 & 0.00 \\
\hline 82 & IEU-COMP-THERM-016-040_ & 0.00 & 0.00 & 0.00 & 0.04 & 0.04 & 0.00 & 0.00 & 0.00 & 0.00 & 0.01 & 0.01 & 0.01 & 0.00 & 0.00 & 0.00 \\
\hline 83 & IEU-COMP-THERM-016-041_- & 0.00 & 0.00 & 0.00 & 0.04 & 0.04 & 0.00 & 0.00 & 0.00 & 0.00 & 0.01 & 0.01 & 0.01 & 0.00 & 0.00 & 0.00 \\
\hline 84 & IEU-COMP-THERM-016-042_- & 0.00 & 0.00 & 0.00 & 0.04 & 0.04 & 0.00 & 0.00 & 0.00 & 0.00 & 0.01 & 0.01 & 0.01 & 0.00 & 0.00 & 0.00 \\
\hline
\end{tabular}


Experiment

IEU-COMP-THERM-016-043 IEU-MET-FAST-002-001_EN IEU-MET-FAST-003-001_EN IEU-MET-FAST-004-001_EN IEU-MET-FAST-005-001_EN IEU-MET-FAST-006-001_EN IEU-MET-FAST-007-001_EN IEU-MET-FAST-008-001 EN IEU-MET-FAST-009-001_EN IEU-MET-FAST-010-001_EN IEU-MET-FAST-012-001_EN IEU-MET-FAST-013-001_EN IEU-MET-FAST-014-001_EN IEU-MET-FAST-014-002 EN IEU-MET-FAST-016-001_EN IEU-MET-FAST-018-002_EN IEU-MET-FAST-019-001 EN IEU-MET-FAST-019-002 EN IEU-MET-FAST-020-001_EN IEU-MET-FAST-020-002 EN IEU-MET-FAST-020-003 EN IEU-MET-FAST-020-004_EN IEU-MET-FAST-020-005 EN IEU-MET-FAST-020-006 EN IEU-MET-FAST-020-007_EN IEU-MET-FAST-021-001_EN IEU-MET-FAST-022-001_EN IEU-MET-FAST-022-005_EN IEU-MET-FAST-022-006 EN IEU-MET-FAST-022-007 EN IEU-MET-INTER-001-002_E IEU-MET-INTER-001-003_E

IEU-MET-INTER-001-004 E IEU-MET-MIXED-001-001_E IEU-SOL-THERM-001-001 E IEU-SOL-THERM-001-002 E IEU-SOL-THERM-001-003_E IEU-SOL-THERM-001-004_E IEU-SOL-THERM-002-001 E IEU-SOL-THERM-002-002_E IEU-SOL-THERM-002-003_E IEU-SOL-THERM-002-004 E

\begin{tabular}{|c|c|c|c|c|c|c|c|c|c|c|c|c|c|}
\hline 2 & 3 & 4 & 5 & b & 1 & 8 & 9 & 10 & 11 & 12 & 13 & 14 & 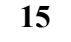 \\
\hline 0.00 & 0.00 & 0.04 & 0.04 & 0.00 & 0.00 & 0.00 & 0.00 & 0.01 & 0.01 & 0.01 & 0.00 & 0.00 & 0.00 \\
\hline 0.40 & .29 & -0.41 & -0.37 & -0.24 & -0.28 & -0.30 & -0.23 & -0.10 & -0.19 & -0.07 & 37 & 13 & .23 \\
\hline 0.12 & -0.01 & -0.15 & -0.14 & -0.08 & -0.11 & 0.07 & -0.07 & 0.03 & 0.00 & 0.08 & .45 & .36 & .54 \\
\hline-0.11 & -0.01 & -0.14 & -0.13 & -0.08 & -0.09 & 0.06 & -0.06 & 0.03 & 0.00 & 0.08 & 0.45 & .37 & .55 \\
\hline-0.14 & -0.04 & -0.13 & -0.12 & -0.05 & -0.07 & -0.04 & -0.02 & 0.05 & 0.01 & 10 & +3 & נJ & .51 \\
\hline-0.08 & 0.02 & -0.12 & -0.11 & -0.06 & -0.08 & -0.05 & -0.05 & 0.04 & 0.01 & 0.08 & 43 & 36 & .53 \\
\hline-0.48 & -0.38 & -0.47 & -0.43 & -0.27 & -0.33 & -0.37 & -0.27 & -0.15 & -0.25 & -0.12 & 0.30 & .01 & .07 \\
\hline-0.07 & 0.03 & -0.11 & -0.10 & -0.06 & -0.08 & -0.04 & -0.05 & 0.05 & 0.02 & 0.09 & 0.45 & 0.39 & 0.57 \\
\hline 0.02 & 0.11 & -0.02 & -0.01 & 0.03 & 0.02 & 0.05 & 0.05 & 0.12 & 0.11 & 0.17 & 0.51 & .46 & .64 \\
\hline-0.48 & -0.39 & -0.47 & -0.42 & -0.27 & & -0.37 & -0.26 & -0.15 & -0.25 & -0.12 & 30 & 01 & .07 \\
\hline .28 & -0.19 & -0.30 & -0.26 & -0.16 & -0.19 & -0.20 & -0.13 & -0.04 & -0.11 & 0.00 & 45 & 25 & .39 \\
\hline 0.44 & -0.34 & -0.44 & -0.40 & -0.25 & -0.30 & -0.34 & -0.24 & -0.13 & -0.22 & -0.10 & 33 & 06 & .14 \\
\hline-0.29 & -0.19 & -0.31 & -0.27 & -0.17 & -0.21 & -0.22 & -0.15 & -0.05 & -0.12 & -0.02 & 0.42 & .23 & .36 \\
\hline-0.14 & -0.06 & -0.18 & -0.15 & -0.09 & -0 . & -0.10 & -0.05 & 0.01 & -0.0 & 0. & 0.4 & 4 & .53 \\
\hline-0.45 & -0.35 & -0.44 & -0.40 & -0.26 & -0 & -0.34 & -0.24 & -0.13 & -0.22 & -0.10 & $r$ & 06 & .14 \\
\hline 0.00 & 0.00 & 0.05 & 0.05 & & & 0.03 & 0.03 & 00 & 0.03 & 0.03 & & & .01 \\
\hline-0.06 & 0.04 & -0.10 & -0.09 & -0.05 & -0 & -0.03 & -0.04 & & 0.03 & 0.10 & 0 & 39 & 0.57 \\
\hline-0.11 & -0.01 & -0.15 & -0.13 & -0.08 & -0 . & -0.07 & -0.07 & 0. & 0.00 & 0.08 & 0 & 6 & 0.54 \\
\hline-0.26 & -0.16 & -0.28 & -0.24 & -0.15 & -0 . & -0.18 & -0.13 & -0 & -0.09 & 0. & & 6 & .40 \\
\hline-0.24 & -0.15 & -0.26 & -0.23 & -0.14 & -0.17 & -0.17 & -0.12 & -0.03 & -0.08 & 0.01 & 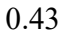 & 27 & .41 \\
\hline 0.25 & -0.15 & -0.26 & -0.23 & -0.14 & -0.17 & -0.17 & -0.12 & -0.03 & -0.09 & 0.01 & 0 & 26 & 0.41 \\
\hline-0.23 & -0.13 & -0.25 & -0.22 & -0.13 & -0.16 & -0.16 & -0.11 & -0.02 & -0.07 & 0.02 & 3 & 8 & 0.43 \\
\hline-0.24 & -0.14 & -0.26 & -0.23 & -0.14 & -0. & -0.17 & -0.11 & -0.03 & -0.08 & 0.0 & 0 . & 0.2 & 0.42 \\
\hline-0.27 & -0.17 & -0.29 & -0.2 & -0.16 & -0 & -0.19 & -0.14 & -0 . & -0.10 & 0.00 & 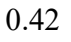 & 0.2 & .39 \\
\hline-0.26 & -0.16 & -0.28 & -0.25 & -0.15 & -0.18 & -0.18 & -0.13 & -0.03 & -0.09 & 0.01 & 0 & .26 & 0.40 \\
\hline-0.33 & -0.22 & -0.34 & -0.31 & -0.19 & & -0.24 & -0.18 & -0.06 & -0.14 & -0.03 & & & 0.34 \\
\hline-0.15 & -0.07 & -0.18 & -0.15 & -0.09 & -0 . & -0.10 & -0.05 & 0.01 & -0.03 & 0.06 & 0.47 & 0.34 & 0.52 \\
\hline-0.09 & -0.01 & -0.12 & -0.09 & -0.04 & -0 . & -0.04 & -0.01 & 0. & 0.0 & 0.09 & 0.5 & 0.40 & 0.58 \\
\hline-0.08 & 0.00 & -0.11 & -0.09 & -0.04 & -0 & -0.04 & -0.01 & 0 & 0.02 & 0.09 & ) & 0 & 0.59 \\
\hline-0.03 & 0.05 & -0.06 & -0.04 & & & 0.01 & & & 0.06 & & & & 0.62 \\
\hline-0.04 & 0.05 & -0.08 & -0.05 & & -0 . & 0.00 & 0.0 & 0. & 0.07 & 0.13 & & 0.44 & 0.62 \\
\hline 0.04 & 0.1 & 0.0 & 0. & & & 0.0 & 0. & 0. & 0. & 0.19 & 0 . & 0.50 & 0.68 \\
\hline 0.05 & 01 & 0.0 & 00 & 0 & & 0.0 & 0.0 & 0 & 0.1 & 0.18 & 0. & 0.50 & 0.68 \\
\hline 0.00 & 0.00 & 0.05 & 0.05 & 0.0 & 0 & 0.02 & 0.02 & 0.02 & 0.02 & 0.02 & 0.00 & 0.01 & 0.01 \\
\hline 0.82 & 0.79 & 0.88 & 0.90 & & & 0.87 & 0.93 & 0.77 & 0.84 & 0.77 & & 0.56 & 0.46 \\
\hline 0.84 & 0.80 & 0.89 & 0.9 & 0 & & 0.87 & 0.93 & 0.7 & 0.82 & 0.74 & 0.41 & 0.55 & 0.47 \\
\hline 0.85 & 0.79 & 0.89 & & 0. & & 0.87 & 0.92 & 0.7 & 0.81 & 0.73 & 0.40 & 0.55 & 0.46 \\
\hline 0.86 & 0.80 & 0.90 & 0.93 & 0.8 & & 0.87 & 0.92 & 0.72 & 0.81 & 0.72 & & & 0.48 \\
\hline 0.86 & 0.77 & 0.85 & 0.89 & 0.76 & & 0.78 & 0.82 & 0.60 & 0.71 & 0.60 & 0.29 & 0.46 & 0.42 \\
\hline 0.84 & 0.77 & 0.84 & 0.88 & 0.77 & & 0.78 & 0.82 & 0.62 & 0.73 & 0.62 & 0.30 & 0.46 & 0.41 \\
\hline 0.82 & 0.77 & 0.83 & 0.86 & 0.77 & 0.82 & 0.77 & 0.81 & 0.65 & 0.74 & 0.64 & 0.32 & 0.47 & 0.41 \\
\hline 0.76 & 0.75 & 0.77 & 0.79 & 0.76 & 0.78 & 0.74 & 0.76 & 0.69 & 0.75 & 0.67 & 0.35 & 0.47 & 0.39 \\
\hline
\end{tabular}


Experiment

IEU-SOL-THERM-002-005_E IEU-SOL-THERM-002-006_E IEU-SOL-THERM-002-007 E IEU-SOL-THERM-002-008_E IEU-SOL-THERM-002-009_E IEU-SOL-THERM-002-010 E IEU-SOL-THERM-002-011_E IEU-SOL-THERM-002-012_E IEU-SOL-THERM-002-013 E IEU-SOL-THERM-003-001_E IEU-SOL-THERM-003-002_E IEU-SOL-THERM-003-003 E IEU-SOL-THERM-003-004_E IEU-SOL-THERM-003-005_E IEU-SOL-THERM-003-006 E IEU-SOL-THERM-003-007_E IEU-SOL-THERM-003-008_E IEU-SOL-THERM-003-009 E IEU-SOL-THERM-003-010_E IEU-SOL-THERM-003-011_E IEU-SOL-THERM-003-012 E IEU-SOL-THERM-003-013_E IEU-SOL-THERM-003-014_E IEU-SOL-THERM-003-015 E IEU-SOL-THERM-003-016_E IEU-SOL-THERM-003-017_E IEU-SOL-THERM-003-018 E IEU-SOL-THERM-003-019_E IEU-SOL-THERM-003-020_E IEU-SOL-THERM-003-021 E IEU-SOL-THERM-003-022_E IEU-SOL-THERM-003-023_E IEU-SOL-THERM-003-024 E IEU-SOL-THERM-003-025_E IEU-SOL-THERM-003-026_E IEU-SOL-THERM-003-027 $\mathrm{E}$ IEU-SOL-THERM-003-028_E IEU-SOL-THERM-003-029_E IEU-SOL-THERM-003-030 E IEU-SOL-THERM-003-031_E IEU-SOL-THERM-003-032_E IEU-SOL-THERM-003-033_E $\begin{array}{ccccc}\mathbf{1} & \mathbf{2} & \mathbf{3} & \mathbf{4} & \mathbf{5}\end{array}$

\begin{tabular}{|c|c|c|c|c|c|c|c|c|c|}
\hline & & & & & & & & & \\
\hline 0.77 & 0.87 & 0.77 & 0.86 & 0.89 & 0.74 & 0.84 & 0.78 & 0.83 & 0.57 \\
\hline 0.81 & 0.91 & 0.82 & 0.89 & 0.92 & 0.76 & 0.86 & 0.81 & 0.84 & 0.58 \\
\hline 0.77 & 0.85 & 0.77 & 0.85 & 0.88 & 0.76 & 0.83 & 0.78 & 0.82 & 0.60 \\
\hline 0.77 & 0.80 & 0.77 & 0.81 & 0.83 & 0.78 & 0.81 & 0.76 & 0.79 & 0.67 \\
\hline 0.68 & 0.62 & 0.67 & 0.65 & 0.65 & 0.70 & 0.67 & 0.65 & 0.65 & 0.70 \\
\hline 0.77 & 0.83 & 0.77 & 0.83 & 0.86 & 0.77 & 0.83 & 0.78 & 0.81 & 0.64 \\
\hline
\end{tabular}

$\begin{array}{llllllllllll}0.77 & 0.83 & 0.77 & 0.83 & 0.86 & 0.77 & 0.83 & 0.78 & 0.81 & 0.64\end{array}$

$\begin{array}{llllllllll}0.75 & 0.76 & 0.75 & 0.78 & 0.80 & 0.77 & 0.78 & 0.74 & 0.76 & 0.69\end{array}$

\begin{tabular}{|l|l|l|l|l|l|l|l|l|l|}
0.78 & 0.83 & 0.77 & 0.83 & 0.86 & 0.77 & 0.83 & 0.78 & 0.81 & 0.65 \\
\hline
\end{tabular}

$\begin{array}{llllllllllll}0.77 & 0.85 & 0.77 & 0.85 & 0.88 & 0.76 & 0.84 & 0.78 & 0.83 & 0.61 \\ 0.81 & 0.90 & 0.82 & 0.90 & 0.93 & 0.78 & 0.87 & 0.83 & 0.86 & 0.61\end{array}$

$\begin{array}{lllllllllll}0.81 & 0.90 & 0.82 & 0.90 & 0.93 & 0.78 & 0.87 & 0.83 & 0.86 & 0.61 \\ 0.79 & 0.89 & 0.80 & 0.89 & 0.92 & 0.77 & 0.87 & 0.82 & 0.86 & 0.60\end{array}$

$\begin{array}{lllllllllll}0.78 & 0.87 & 0.78 & 0.88 & 0.91 & 0.77 & 0.86 & 0.81 & 0.85 & 0.60\end{array}$

\begin{tabular}{|l|l|l|l|l|l|l|l|l|l|}
0.77 & 0.86 & 0.77 & 0.87 & 0.90 & 0.77 & 0.85 & 0.80 & 0.84 & 0.60 \\
\hline
\end{tabular}

\begin{tabular}{l|l|l|l|l|l|l|l|l|l|}
0.77 & 0.85 & 0.77 & 0.86 & 0.89 & 0.78 & 0.85 & 0.80 & 0.84 & 0.62 \\
\hline
\end{tabular}

$\begin{array}{lllllllllll}0.78 & 0.84 & 0.78 & 0.85 & 0.88 & 0.78 & 0.85 & 0.79 & 0.84 & 0.64 \\ 0.78 & 0.83 & 0.77 & 0.84 & 0.87 & 0.79 & 0.84 & 0.79 & 0.83 & 0.66\end{array}$

$\begin{array}{llllllllllll}0.78 & 0.83 & 0.77 & 0.84 & 0.87 & 0.79 & 0.84 & 0.79 & 0.83 & 0.66 \\ 0.77 & 0.81 & 0.77 & 0.82 & 0.85 & 0.79 & 0.83 & 0.78 & 0.82 & 0.68\end{array}$

\begin{tabular}{lllll|l|l|l|l|l|}
0.77 & 0.87 & 0.78 & 0.87 & 0.91 & 0.77 & 0.86 & 0.80 & 0.85 & 0.60
\end{tabular}

\begin{tabular}{l|l|l|l|l|l|l|l|l|l|}
0.78 & 0.83 & 0.78 & 0.84 & 0.87 & 0.79 & 0.84 & 0.79 & 0.83 & 0.66 \\
\hline
\end{tabular}

\begin{tabular}{llll|l|l|lllll}
0.80 & 0.90 & 0.81 & 0.89 & 0.93 & 0.77 & 0.87 & 0.82 & 0.86 & 0.60
\end{tabular}

$\begin{array}{lllllllllll}0.80 & 0.90 & 0.81 & 0.89 & 0.93 & 0.78 & 0.87 & 0.82 & 0.86 & 0.60 \\ 0.79 & 0.89 & 0.80 & 0.89 & 0.92 & 0.77 & 0.86 & 0.82 & 0.86 & 0.60\end{array}$

$\begin{array}{lllllllllll}0.79 & 0.89 & 0.80 & 0.89 & 0.92 & 0.77 & 0.86 & 0.82 & 0.86 & 0.60 \\ 0.78 & 0.88 & 0.79 & 0.88 & 0.91 & 0.77 & 0.86 & 0.81 & 0.85 & 0.59\end{array}$

$\begin{array}{llllllllllll}0.78 & 0.87 & 0.78 & 0.87 & 0.91 & 0.76 & 0.85 & 0.80 & 0.85 & 0.59\end{array}$

\begin{tabular}{l|l|l|l|l|l|l|l|l|l|}
0.77 & 0.86 & 0.77 & 0.87 & 0.90 & 0.77 & 0.85 & 0.80 & 0.84 & 0.60 \\
\hline
\end{tabular}

\begin{tabular}{llllllllllll}
0.77 & 0.86 & 0.77 & 0.86 & 0.90 & 0.77 & 0.85 & 0.80 & 0.84 & 0.61 \\
\hline
\end{tabular}

$\begin{array}{lllllllllll}0.77 & 0.85 & 0.77 & 0.86 & 0.89 & 0.78 & 0.85 & 0.80 & 0.84 & 0.62 \\ 0.77 & 0.85 & 0.77 & 0.86 & 0.89 & 0.78 & 0.85 & 0.80 & 0.84 & 0.63\end{array}$

$\begin{array}{lllllllllll}0.77 & 0.85 & 0.77 & 0.86 & 0.89 & 0.78 & 0.85 & 0.80 & 0.84 & 0.63 \\ 0.78 & 0.84 & 0.78 & 0.85 & 0.88 & 0.79 & 0.85 & 0.80 & 0.84 & 0.65\end{array}$

$\begin{array}{llllllllllll}0.78 & 0.84 & 0.78 & 0.85 & 0.88 & 0.79 & 0.85 & 0.80 & 0.84 & 0.66\end{array}$

$\begin{array}{llllllllllll}0.78 & 0.88 & 0.79 & 0.88 & 0.91 & 0.76 & 0.85 & 0.80 & 0.84 & 0.59 \\ 0.77 & 0.86 & 0.78 & 0.86 & 0.89 & 0.77 & 0.85 & 0.79 & 0.84 & 0.60\end{array}$

\begin{tabular}{|l|l|l|l|l|l|l|l|l|l|}
0.77 & 0.86 & 0.78 & 0.86 & 0.89 & 0.77 & 0.85 & 0.79 & 0.84 & 0.60 \\
\hline
\end{tabular}

$\begin{array}{lllllllllll}0.81 & 0.90 & 0.82 & 0.90 & 0.93 & 0.80 & 0.88 & 0.84 & 0.88 & 0.63 \\ 0.80 & 0.89 & 0.81 & 0.89 & 0.93 & 0.79 & 0.88 & 0.83 & 0.87 & 0.62\end{array}$

$\begin{array}{llllllllll}0.80 & 0.89 & 0.80 & 0.89 & 0.92 & 0.79 & 0.87 & 0.83 & 0.87 & 0.62\end{array}$

$\begin{array}{lllllllllll}0.79 & 0.88 & 0.80 & 0.89 & 0.92 & 0.79 & 0.87 & 0.82 & 0.87 & 0.62\end{array}$

$\begin{array}{llllllllll}0.79 & 0.88 & 0.80 & 0.89 & 0.92 & 0.79 & 0.87 & 0.82 & 0.87 & 0.62 \\ 0.79 & 0.88 & 0.79 & 0.88 & 0.92 & 0.79 & 0.87 & 0.82 & 0.87 & 0.62\end{array}$

\begin{tabular}{llllllllllll}
0.78 & 0.87 & 0.79 & 0.88 & 0.91 & 0.78 & 0.87 & 0.82 & 0.86 & 0.62 \\
\hline
\end{tabular}

$\begin{array}{lllllllllll}0.78 & 0.87 & 0.78 & 0.88 & 0.91 & 0.79 & 0.87 & 0.81 & 0.86 & 0.62 \\ 0.81 & 0.91 & 0.82 & 0.90 & 0.93 & 0.77 & 0.87 & 0.82 & 0.86 & 0.60\end{array}$

$\begin{array}{lllllllllll}0.79 & 0.89 & 0.80 & 0.88 & 0.92 & 0.76 & 0.86 & 0.81 & 0.85 & 0.59\end{array}$

\begin{tabular}{l|l|l|l|l|l|l|l|l|l}
0.78 & 0.87 & 0.78 & 0.87 & 0.91 & 0.76 & 0.85 & 0.80 & 0.84 & 0.59
\end{tabular}

$\begin{array}{lllll}11 & 12 & 13 & 14 & 15\end{array}$ $\begin{array}{lllll}0.70 & 0.58 & 0.28 & 0.47 & 0.44 \\ 0.72 & 0.60 & 0.32 & 0.54 & 0.53\end{array}$ $\begin{array}{lllll}0.72 & 0.61 & 0.29 & 0.46 & 0.42\end{array}$ $\begin{array}{lllll}0.75 & 0.66 & 0.34 & 0.47 & 0.40\end{array}$ $\begin{array}{lllll}0.71 & 0.68 & 0.38 & 0.45 & 0.34\end{array}$ $\begin{array}{lllll}0.74 & 0.64 & 0.31 & 0.47 & 0.41\end{array}$ $\begin{array}{lllll}0.75 & 0.68 & 0.35 & 0.47 & 0.39\end{array}$ $\begin{array}{lllll}0.74 & 0.64 & 0.32 & 0.47 & 0.41\end{array}$ 
Experiment

IEU-SOL-THERM-003-034_E IEU-SOL-THERM-003-035_E IEU-SOL-THERM-003-036 E IEU-SOL-THERM-003-037_E IEU-SOL-THERM-003-038_E IEU-SOL-THERM-003-039 E IEU-SOL-THERM-003-040_E IEU-SOL-THERM-003-041_E IEU-SOL-THERM-003-042 E IEU-SOL-THERM-003-043_E IEU-SOL-THERM-003-044_E IEU-SOL-THERM-003-045 E IEU-SOL-THERM-003-046_E LEU-COMP-FAST-001-003_E LEU-COMP-MIXED-001-001 LEU-COMP-MIXED-001-002 LEU-COMP-MIXED-002-001_ LEU-COMP-MIXED-002-002 LEU-COMP-MIXED-002-003 LEU-COMP-THERM-001-001 LEU-COMP-THERM-001-002 LEU-COMP-THERM-001-003 LEU-COMP-THERM-001-004 LEU-COMP-THERM-001-005 LEU-COMP-THERM-001-006 LEU-COMP-THERM-001-007 LEU-COMP-THERM-001-008 LEU-COMP-THERM-002-001 LEU-COMP-THERM-002-002 LEU-COMP-THERM-002-003 LEU-COMP-THERM-002-004 LEU-COMP-THERM-002-005 LEU-COMP-THERM-003-001 LEU-COMP-THERM-003-002 LEU-COMP-THERM-003-003 LEU-COMP-THERM-003-004 LEU-COMP-THERM-003-005 LEU-COMP-THERM-003-006 LEU-COMP-THERM-003-007 LEU-COMP-THERM-003-008 LEU-COMP-THERM-003-009 LEU-COMP-THERM-003-010

\begin{tabular}{|c|c|c|c|c|c|c|c|c|c|c|c|c|c|c|}
\hline 1 & 2 & 3 & 4 & 5 & 6 & 7 & 8 & 9 & 10 & 11 & 12 & 13 & 14 & 15 \\
\hline 0.77 & 0.86 & 0.77 & 0.86 & 0.90 & 0.76 & 0.84 & 0.79 & 0.84 & 0.59 & 0.71 & 0.59 & 0.28 & 0.47 & 0.44 \\
\hline 0.77 & 0.85 & 0.77 & 0.86 & 0.89 & 0.76 & 0.84 & 0.79 & 0.83 & 0.60 & 0.72 & 0.61 & 0.29 & 0.46 & 0.42 \\
\hline 0.77 & 0.84 & 0.77 & 0.85 & 0.88 & 0.77 & 0.84 & 0.78 & 0.83 & 0.63 & 0.73 & 0.63 & 0.30 & 0.47 & 0.42 \\
\hline 0.78 & 0.83 & 0.77 & 0.84 & 0.87 & 0.78 & 0.83 & 0.78 & 0.82 & 0.65 & 0.74 & 0.64 & 0.32 & 0.47 & 0.41 \\
\hline 0.80 & 0.90 & 0.81 & 0.89 & 0.92 & 0.76 & 0.86 & 0.81 & 0.85 & 0.58 & 0.72 & 0.60 & 0.32 & 0.53 & 0.52 \\
\hline 0.79 & 0.89 & 0.80 & 0.88 & 0.91 & 0.76 & 0.85 & 0.80 & 0.84 & 0.58 & 0.71 & 0.59 & 0.30 & 0.51 & 0.49 \\
\hline 0.78 & 0.88 & 0.79 & 0.87 & 0.91 & 0.75 & 0.85 & 0.80 & 0.84 & 0.57 & 0.70 & 0.58 & 0.29 & 0.49 & 0.47 \\
\hline 0.78 & 0.88 & 0.78 & 0.87 & 0.90 & 0.75 & 0.84 & 0.79 & 0.83 & 0.57 & 0.70 & 0.58 & 0.29 & 0.48 & 0.46 \\
\hline 0.77 & 0.86 & 0.77 & 0.86 & 0.89 & 0.75 & 0.84 & 0.78 & 0.83 & 0.58 & 0.70 & 0.58 & 0.28 & 0.47 & 0.44 \\
\hline 0.77 & 0.86 & 0.77 & 0.86 & 0.89 & 0.76 & 0.84 & 0.78 & 0.83 & 0.59 & 0.71 & 0.59 & 0.28 & 0.46 & 0.43 \\
\hline 0.77 & 0.86 & 0.77 & 0.85 & 0.89 & 0.76 & 0.84 & 0.78 & 0.83 & 0.60 & 0.71 & 0.60 & 0.29 & 0.46 & 0.42 \\
\hline 0.78 & 0.88 & 0.79 & 0.87 & 0.91 & 0.75 & 0.85 & 0.80 & 0.84 & 0.57 & 0.71 & 0.59 & 0.29 & 0.49 & 0.47 \\
\hline 0.77 & 0.85 & 0.77 & 0.85 & 0.89 & 0.76 & 0.84 & 0.78 & 0.83 & 0.60 & 0.71 & 0.60 & 0.29 & 0.46 & 0.42 \\
\hline 0.14 & 0.11 & 0.19 & 0.06 & 0.07 & 0.08 & 0.08 & 0.12 & 0.11 & 0.14 & 0.15 & 0.19 & 0.50 & 0.49 & 0.68 \\
\hline 0.15 & 0.10 & 0.20 & 0.05 & 0.06 & 0.10 & 0.09 & 0.12 & 0.11 & 0.18 & 0.18 & 0.23 & 0.56 & 0.52 & 0.69 \\
\hline 0.15 & 0.11 & 0.20 & 0.06 & 0.07 & 0.10 & 0.09 & 0.13 & 0.12 & 0.17 & 0.17 & 0.22 & 0.54 & 0.51 & 0.69 \\
\hline 0.03 & 0.03 & 0.03 & 0.01 & 0.01 & 0.00 & 0.00 & 0.00 & 0.00 & 0.01 & 0.01 & 0.01 & 0.00 & 0.00 & 0.00 \\
\hline 0.04 & 0.04 & 0.04 & 0.02 & 0.02 & 0.00 & 0.00 & 0.00 & 0.00 & 0.01 & 0.01 & 0.01 & 0.00 & 0.00 & 0.00 \\
\hline 0.05 & 0.05 & 0.05 & 0.01 & 0.01 & 0.00 & 0.00 & 0.00 & 0.00 & 0.01 & 0.01 & 0.01 & 0.00 & 0.00 & 0.00 \\
\hline 0.96 & 0.90 & 0.94 & 0.89 & 0.87 & 0.90 & 0.89 & 0.89 & 0.81 & 0.85 & 0.90 & 0.82 & 0.54 & 0.68 & 0.55 \\
\hline 0.95 & 0.88 & 0.93 & 0.88 & 0.85 & 0.89 & 0.88 & 0.88 & 0.80 & 0.86 & 0.90 & 0.83 & 0.55 & 0.69 & 0.54 \\
\hline 0.94 & 0.87 & 0.92 & 0.87 & 0.84 & 0.89 & 0.87 & 0.87 & 0.79 & 0.86 & 0.90 & 0.83 & 0.56 & 0.68 & 0.54 \\
\hline 0.95 & 0.88 & 0.93 & 0.88 & 0.85 & 0.89 & 0.87 & 0.88 & 0.80 & 0.86 & 0.90 & 0.83 & 0.55 & 0.69 & 0.54 \\
\hline 0.94 & 0.86 & 0.92 & 0.86 & 0.83 & 0.89 & 0.86 & 0.87 & 0.78 & 0.86 & 0.90 & 0.83 & 0.56 & 0.68 & 0.53 \\
\hline 0.95 & 0.87 & 0.93 & 0.87 & 0.85 & 0.89 & 0.87 & 0.87 & 0.79 & 0.86 & 0.90 & 0.83 & 0.56 & 0.68 & 0.54 \\
\hline 0.93 & 0.86 & 0.91 & 0.86 & 0.83 & 0.89 & 0.86 & 0.86 & 0.78 & 0.86 & 0.90 & 0.83 & 0.56 & 0.68 & 0.53 \\
\hline 0.94 & 0.86 & 0.92 & 0.86 & 0.84 & 0.89 & 0.86 & 0.87 & 0.78 & 0.86 & 0.90 & 0.83 & 0.56 & 0.68 & 0.53 \\
\hline 0.96 & 0.97 & 0.95 & 0.95 & 0.95 & 0.89 & 0.92 & 0.91 & 0.86 & 0.78 & 0.88 & 0.77 & 0.44 & 0.64 & 0.55 \\
\hline 0.96 & 0.96 & 0.95 & 0.95 & 0.95 & 0.89 & 0.92 & 0.91 & 0.86 & 0.79 & 0.88 & 0.77 & 0.44 & 0.64 & 0.55 \\
\hline 0.96 & 0.96 & 0.95 & 0.95 & 0.94 & 0.89 & 0.92 & 0.91 & 0.86 & 0.79 & 0.88 & 0.78 & 0.44 & 0.64 & 0.55 \\
\hline 0.96 & 0.96 & 0.96 & 0.94 & 0.94 & 0.89 & 0.92 & 0.91 & 0.86 & 0.80 & 0.89 & 0.79 & 0.46 & 0.65 & 0.55 \\
\hline 0.96 & 0.95 & 0.95 & 0.94 & 0.93 & 0.90 & 0.91 & 0.91 & 0.86 & 0.81 & 0.89 & 0.80 & 0.47 & 0.65 & 0.55 \\
\hline 0.96 & 0.88 & 0.95 & 0.87 & 0.83 & 0.88 & 0.86 & 0.88 & 0.77 & 0.86 & 0.90 & 0.83 & 0.59 & 0.73 & 0.58 \\
\hline 0.97 & 0.89 & 0.95 & 0.88 & 0.84 & 0.88 & 0.86 & 0.88 & 0.77 & 0.85 & 0.90 & 0.83 & 0.58 & 0.73 & 0.58 \\
\hline 0.97 & 0.89 & 0.95 & 0.87 & 0.84 & 0.88 & 0.86 & 0.88 & 0.77 & 0.86 & 0.90 & 0.83 & 0.59 & 0.73 & 0.58 \\
\hline 0.97 & 0.89 & 0.95 & 0.87 & 0.84 & 0.88 & 0.86 & 0.88 & 0.77 & 0.86 & 0.90 & 0.83 & 0.59 & 0.73 & 0.58 \\
\hline 0.96 & 0.88 & 0.95 & 0.87 & 0.83 & 0.88 & 0.86 & 0.88 & 0.77 & 0.86 & 0.90 & 0.84 & 0.59 & 0.73 & 0.58 \\
\hline 0.97 & 0.89 & 0.95 & 0.88 & 0.85 & 0.89 & 0.87 & 0.89 & 0.78 & 0.86 & 0.91 & 0.84 & 0.57 & 0.72 & 0.57 \\
\hline 0.97 & 0.89 & 0.95 & 0.88 & 0.85 & 0.89 & 0.87 & 0.89 & 0.78 & 0.86 & 0.91 & 0.83 & 0.58 & 0.72 & 0.58 \\
\hline 0.97 & 0.89 & 0.95 & 0.88 & 0.85 & 0.89 & 0.87 & 0.89 & 0.78 & 0.86 & 0.91 & 0.83 & 0.57 & 0.72 & 0.57 \\
\hline 0.96 & 0.87 & 0.94 & 0.86 & 0.83 & 0.89 & 0.86 & 0.87 & 0.77 & 0.87 & 0.91 & 0.84 & 0.59 & 0.72 & 0.57 \\
\hline 0.97 & 0.89 & 0.95 & 0.87 & 0.84 & 0.89 & 0.87 & 0.88 & 0.78 & 0.86 & 0.91 & 0.84 & 0.58 & 0.72 & 0.57 \\
\hline
\end{tabular}


Experiment

LEU-COMP-THERM-003-011 LEU-COMP-THERM-003-012 LEU-COMP-THERM-003-013 LEU-COMP-THERM-003-014 LEU-COMP-THERM-003-015 LEU-COMP-THERM-003-016 LEU-COMP-THERM-003-017 LEU-COMP-THERM-003-018 LEU-COMP-THERM-003-019 LEU-COMP-THERM-003-020 LEU-COMP-THERM-003-021 LEU-COMP-THERM-003-022 LEU-COMP-THERM-004-001 LEU-COMP-THERM-004-002 LEU-COMP-THERM-004-003 LEU-COMP-THERM-004-004 LEU-COMP-THERM-004-005 LEU-COMP-THERM-004-006 LEU-COMP-THERM-004-007 LEU-COMP-THERM-004-008 LEU-COMP-THERM-004-009 LEU-COMP-THERM-004-010 LEU-COMP-THERM-004-011 LEU-COMP-THERM-004-012 LEU-COMP-THERM-004-013 LEU-COMP-THERM-004-014 LEU-COMP-THERM-004-015 LEU-COMP-THERM-004-016 LEU-COMP-THERM-004-017 LEU-COMP-THERM-004-018 LEU-COMP-THERM-004-019 LEU-COMP-THERM-004-020 LEU-COMP-THERM-005-001 LEU-COMP-THERM-005-002 LEU-COMP-THERM-005-003 LEU-COMP-THERM-005-004 LEU-COMP-THERM-005-005 LEU-COMP-THERM-005-006 LEU-COMP-THERM-005-007 LEU-COMP-THERM-005-008 LEU-COMP-THERM-005-009 LEU-COMP-THERM-005-010

\begin{tabular}{|c|c|c|c|c|c|c|c|c|c|c|c|c|c|c|}
\hline 1 & 2 & 3 & 4 & 5 & 6 & 7 & 8 & 9 & 10 & 11 & 12 & 13 & 14 & 15 \\
\hline 0.94 & 0.84 & 0.92 & 0.83 & 0.80 & 0.88 & 0.84 & 0.85 & 0.75 & 0.88 & 0.90 & 0.85 & 0.61 & 0.72 & 55 \\
\hline 0.88 & 0.74 & 0.85 & 0.76 & 0.73 & 0.85 & 0.79 & 0.80 & 0.71 & 0.89 & 0.88 & 0.86 & 0.65 & 0.71 & \\
\hline 0.96 & 0.87 & 0.94 & 0.86 & 0.83 & 0.89 & 0.86 & 0.87 & 0.77 & 0.87 & 0.91 & 0.84 & 0.59 & 0.72 & \\
\hline 0.96 & 0.87 & 0.94 & 0.86 & 0.83 & 0.89 & 0.86 & 0.87 & 0.77 & 0.87 & 0.91 & 0.84 & 0.59 & 0.72 & \\
\hline 0.95 & 0.87 & 0.93 & 0.86 & 0.82 & 0.88 & 0.86 & 0.87 & 0.77 & 0.87 & 0.90 & 0.84 & 0.59 & 0.72 & \\
\hline 0.95 & 0.86 & 0.93 & 0.85 & 0.82 & 0.88 & 0.85 & 0.87 & 0.76 & 0.87 & 0.91 & 0.85 & 0.60 & 0.72 & \\
\hline 0.95 & 0.85 & 0.93 & 0.84 & 0.80 & 0.88 & 0.84 & 0.86 & 0.75 & 0.88 & 0.90 & 0.85 & 0.61 & 0.72 & \\
\hline 0.94 & 0.84 & 0.92 & 0.84 & 0.80 & 0.88 & 0.84 & 0.86 & 0.75 & 0.88 & 0.90 & 0.85 & 0.61 & 0.72 & \\
\hline 0.95 & 0.85 & 0.93 & 0.84 & 0.81 & 0.88 & 0.84 & 0.86 & 0.75 & 0.87 & 0.90 & 0.85 & 0.60 & 0.72 & \\
\hline 0.96 & 0.87 & 0.94 & 0.85 & 0.82 & 0.88 & 0.85 & 0.87 & 0.76 & 0.87 & 0.90 & 0.84 & 0.60 & 0.73 & \\
\hline 0.95 & 0.86 & 0.93 & 0.85 & 0.81 & 0.88 & 0.85 & 0.87 & 0.76 & 0.87 & 0.90 & 0.85 & 0.61 & 0.73 & \\
\hline 0.95 & 0.84 & 0.93 & 0.84 & 0.80 & 0.88 & 0.84 & 0.86 & 0.75 & 0.87 & 0.90 & 0.84 & 0.61 & 0.73 & \\
\hline 0.99 & 0.97 & 0.98 & 0.94 & 0.92 & 0.87 & 0.90 & 0.92 & 0.82 & 0.80 & 0.89 & 0.79 & 0.49 & 0.71 & \\
\hline 0.99 & 0.96 & 0.98 & 0.93 & 0.91 & 0.88 & 0.90 & 0.92 & 0.82 & 0.80 & 0.89 & 0.80 & 0.51 & 0.71 & \\
\hline 0.99 & 0.97 & 0.98 & 0.94 & 0.92 & 0.88 & 0.90 & 0.92 & 0.82 & 0.80 & 0.89 & 0.79 & 0.50 & 0.71 & \\
\hline 0.99 & 0.96 & 0.98 & 0.93 & 0.91 & 0.88 & 0.90 & 0.92 & 0.82 & 0.80 & 0.89 & 0.80 & 0.51 & 0.71 & \\
\hline 0.98 & 0.98 & 0.98 & 0.95 & 0.94 & 0.88 & 0.91 & 0.92 & 0.85 & 0.79 & 0.89 & 0.78 & 0.46 & 0.68 & \\
\hline 0.99 & 0.97 & 0.98 & 0.95 & 0.93 & 0.88 & 0.91 & 0.92 & 0.84 & 0.80 & 0.89 & 0.79 & 0.48 & 0.69 & \\
\hline 0.99 & 0.97 & 0.98 & 0.95 & 0.93 & 0.89 & 0.91 & 0.92 & 0.84 & 0.81 & 0.90 & 0.80 & 0.48 & 0.69 & \\
\hline 0.99 & 0.96 & 0.98 & 0.94 & 0.92 & 0.88 & 0.90 & 0.92 & 0.83 & 0.81 & 0.90 & 0.80 & 0.50 & 0.70 & \\
\hline 0.99 & 0.94 & 0.98 & 0.92 & 0.90 & 0.88 & 0.89 & 0.91 & 0.82 & 0.83 & 0.91 & 0.82 & 0.53 & 0.72 & \\
\hline 0.99 & 0.94 & 0.98 & 0.92 & 0.90 & 0.89 & 0.89 & 0.91 & 0.82 & 0.84 & 0.91 & 0.82 & 0.54 & 0.72 & \\
\hline 0.98 & 0.92 & 0.97 & 0.91 & 0.88 & 0.89 & 0.89 & 0.90 & 0.82 & 0.85 & 0.92 & 0.84 & 0.54 & 0.71 & \\
\hline 0.96 & 0.89 & 0.95 & 0.88 & 0.86 & 0.89 & 0.87 & 0.89 & 0.80 & 0.87 & 0.92 & 0.85 & 0.57 & 0.72 & \\
\hline 0.99 & 0.96 & 0.98 & 0.94 & 0.92 & 0.89 & 0.90 & 0.92 & 0.83 & 0.82 & 0.90 & 0.81 & 0.51 & 0.70 & \\
\hline 0.99 & 0.96 & 0.98 & 0.94 & 0.92 & 0.88 & 0.90 & 0.92 & 0.83 & 0.82 & 0.90 & 0.81 & 0.50 & 0.70 & \\
\hline 0.99 & 0.96 & 0.98 & 0.94 & 0.92 & 0.89 & 0.90 & 0.92 & 0.83 & 0.82 & 0.90 & 0.81 & 0.50 & 0.70 & \\
\hline 0.99 & 0.96 & 0.98 & 0.94 & 0.92 & 0.89 & 0.91 & 0.92 & 0.84 & 0.81 & 0.90 & 0.81 & 0.50 & 0.70 & \\
\hline 0.99 & 0.95 & 0.98 & 0.93 & 0.91 & 0.89 & 0.90 & 0.92 & 0.83 & 0.83 & 0.91 & 0.82 & 0.51 & 0.71 & \\
\hline 0.99 & 0.96 & 0.98 & 0.94 & 0.92 & 0.89 & 0.90 & 0.92 & 0.83 & 0.82 & 0.90 & 0.81 & 0.51 & 0.71 & \\
\hline 0.99 & 0.95 & 0.98 & 0.93 & 0.91 & 0.89 & 0.90 & 0.91 & 0.82 & 0.83 & 0.91 & 0.82 & 0.52 & 0.71 & \\
\hline 0.98 & 0.93 & 0.97 & 0.91 & 0.89 & 0.89 & 0.89 & 0.91 & 0.82 & 0.85 & 0.91 & 0.84 & 0.53 & 0.71 & \\
\hline 0.98 & 0.98 & 0.97 & 0.96 & 0.95 & 0.89 & 0.92 & 0.92 & 0.86 & 0.79 & 0.88 & 0.78 & 0.45 & 0.66 & \\
\hline 0.96 & 0.95 & 0.95 & 0.93 & 0.92 & 0.87 & 0.90 & 0.90 & 0.84 & 0.80 & 0.89 & 0.81 & 0.46 & 0.66 & \\
\hline 0.63 & 0.56 & 0.62 & 0.55 & 0.53 & 0.59 & 0.57 & 0.58 & 0.52 & 0.66 & 0.68 & 0.72 & 0.42 & 0.49 & \\
\hline 0.59 & 0.51 & 0.58 & 0.51 & 0.49 & 0.55 & 0.53 & 0.54 & 0.48 & 0.64 & 0.64 & 0.69 & 0.41 & 0.47 & \\
\hline 0.96 & 0.89 & 0.96 & 0.86 & 0.83 & 0.84 & 0.84 & 0.87 & 0.75 & 0.81 & 0.87 & 0.80 & 0.59 & 0.76 & \\
\hline 0.94 & 0.85 & 0.93 & 0.82 & 0.78 & 0.82 & 0.80 & 0.84 & 0.71 & 0.82 & 0.87 & 0.82 & 0.62 & 0.77 & \\
\hline 0.87 & 0.77 & 0.87 & 0.74 & 0.70 & 0.76 & 0.74 & 0.78 & 0.65 & 0.81 & 0.83 & 0.82 & 0.63 & 0.75 & \\
\hline 0.73 & 0.60 & 0.73 & 0.58 & 0.54 & 0.66 & 0.61 & 0.64 & 0.53 & 0.76 & 0.75 & 0.79 & 0.63 & 0.68 & \\
\hline 0.63 & 0.49 & 0.63 & 0.47 & 0.44 & 0.58 & 0.52 & 0.55 & 0.44 & 0.71 & 0.68 & 0.75 & 0.62 & 0.62 & \\
\hline 0.56 & 0.42 & 0.56 & 0.40 & 0.37 & 0.53 & 0.46 & 0.49 & 0.40 & 0.68 & 0.64 & 0.72 & 0.61 & 0.59 & \\
\hline
\end{tabular}


Experiment

LEU-COMP-THERM-005-011_ LEU-COMP-THERM-005-012 LEU-COMP-THERM-005-013 LEU-COMP-THERM-005-014_LEU-COMP-THERM-005-015 LEU-COMP-THERM-005-016 LEU-COMP-THERM-006-001_ LEU-COMP-THERM-006-002 LEU-COMP-THERM-006-003 LEU-COMP-THERM-006-004 LEU-COMP-THERM-006-005 LEU-COMP-THERM-006-006 LEU-COMP-THERM-006-007 LEU-COMP-THERM-006-008 EU-COMP-THERM-006-009 LEU-COMP-THERM-006-010 LEU-COMP-THERM-006-011_ LEU-COMP-THERM-006-012 LEU-COMP-THERM-006-013 LEU-COMP-THERM-006-014 LEU-COMP-THERM-006-015 LEU-COMP-THERM-006-016 LEU-COMP-THERM-006-017 LEU-COMP-THERM-006-018 LEU-COMP-THERM-008-001_ LEU-COMP-THERM-008-002 LEU-COMP-THERM-008-003 LEU-COMP-THERM-008-004 LEU-COMP-THERM-008-005 LEU-COMP-THERM-008-006 LEU-COMP-THERM-008-007 LEU-COMP-THERM-008-008 LEU-COMP-THERM-008-009 LEU-COMP-THERM-008-010 LEU-COMP-THERM-008-011 LEU-COMP-THERM-008-012 LEU-COMP-THERM-008-013 LEU-COMP-THERM-008-014 LEU-COMP-THERM-008-015 LEU-COMP-THERM-008-016 LEU-COMP-THERM-008-017 LEU-COMP-THERM-009-001

\begin{tabular}{|c|c|c|c|c|c|c|c|c|c|c|c|c|c|c|}
\hline 1 & 2 & 3 & 4 & 5 & 6 & 7 & 8 & 9 & 10 & 11 & 12 & 13 & 14 & 15 \\
\hline 0.56 & 0.42 & 0.56 & 0.40 & 0.37 & 0.53 & 0.46 & 0.49 & 0.39 & 0.67 & 0.63 & 0.71 & 0.60 & 0.58 & 0.44 \\
\hline 0.66 & 0.47 & 0.66 & 0.44 & 0.40 & 0.62 & 0.52 & 0.56 & 0.44 & 0.74 & 0.68 & 0.72 & 0.84 & 0.77 & 0.60 \\
\hline 0.61 & 0.42 & 0.61 & 0.39 & 0.35 & 0.58 & 0.48 & 0.52 & 0.40 & 0.72 & 0.65 & 0.71 & 0.84 & 0.76 & 0.59 \\
\hline 0.97 & 0.91 & 0.96 & 0.90 & 0.87 & 0.89 & 0.88 & 0.89 & 0.79 & 0.85 & 0.90 & 0.82 & 0.56 & 0.72 & 0.58 \\
\hline 0.87 & 0.76 & 0.85 & 0.75 & 0.72 & 0.81 & 0.77 & 0.79 & 0.68 & 0.85 & 0.87 & 0.86 & 0.59 & 0.68 & 0.52 \\
\hline 0.89 & 0.75 & 0.87 & 0.74 & 0.69 & 0.81 & 0.76 & 0.79 & 0.65 & 0.85 & 0.85 & 0.81 & 0.69 & 0.76 & 0.58 \\
\hline 0.97 & 0.89 & 0.95 & 0.87 & 0.84 & 0.87 & 0.86 & 0.88 & 0.76 & 0.84 & 0.89 & 0.82 & 0.58 & 0.73 & 0.60 \\
\hline 0.97 & 0.89 & 0.95 & 0.87 & 0.83 & 0.87 & 0.85 & 0.88 & 0.76 & 0.84 & 0.89 & 0.81 & 0.58 & 0.73 & 0.60 \\
\hline 0.97 & 0.89 & 0.95 & 0.87 & 0.83 & 0.87 & 0.85 & 0.88 & 0.76 & 0.84 & 0.89 & 0.81 & 0.58 & 0.73 & 0.60 \\
\hline 0.98 & 0.91 & 0.96 & 0.90 & 0.87 & 0.89 & 0.88 & 0.90 & 0.79 & 0.84 & 0.90 & 0.82 & 0.56 & 0.72 & 0.59 \\
\hline 0.98 & 0.92 & 0.96 & 0.90 & 0.87 & 0.88 & 0.88 & 0.90 & 0.79 & 0.84 & 0.90 & 0.81 & 0.55 & 0.72 & 0.59 \\
\hline 0.98 & 0.92 & 0.96 & 0.90 & 0.87 & 0.88 & 0.88 & 0.90 & 0.79 & 0.84 & 0.90 & 0.81 & 0.55 & 0.72 & 0.59 \\
\hline 0.98 & 0.92 & 0.96 & 0.90 & 0.87 & 0.88 & 0.88 & 0.90 & 0.79 & 0.83 & 0.90 & 0.81 & 0.55 & 0.72 & 0.59 \\
\hline 0.98 & 0.92 & 0.96 & 0.90 & 0.87 & 0.88 & 0.88 & 0.90 & 0.79 & 0.83 & 0.89 & 0.81 & 0.55 & 0.72 & 0.59 \\
\hline 0.98 & 0.93 & 0.96 & 0.91 & 0.89 & 0.90 & 0.89 & 0.90 & 0.81 & 0.84 & 0.90 & 0.82 & 0.53 & 0.70 & 0.57 \\
\hline 0.98 & 0.93 & 0.96 & 0.92 & 0.89 & 0.90 & 0.89 & 0.91 & 0.81 & 0.84 & 0.90 & 0.81 & 0.53 & 0.70 & 0.57 \\
\hline 0.98 & 0.93 & 0.96 & 0.92 & 0.89 & 0.90 & 0.89 & 0.91 & 0.81 & 0.84 & 0.90 & 0.81 & 0.53 & 0.70 & 0.57 \\
\hline 0.98 & 0.93 & 0.96 & 0.92 & 0.89 & 0.90 & 0.89 & 0.91 & 0.81 & 0.84 & 0.90 & 0.81 & 0.53 & 0.70 & 0.57 \\
\hline 0.98 & 0.93 & 0.96 & 0.91 & 0.89 & 0.89 & 0.89 & 0.90 & 0.81 & 0.84 & 0.90 & 0.81 & 0.53 & 0.70 & 0.57 \\
\hline 0.97 & 0.92 & 0.95 & 0.91 & 0.89 & 0.90 & 0.90 & 0.90 & 0.82 & 0.84 & 0.90 & 0.82 & 0.53 & 0.69 & 0.56 \\
\hline 0.97 & 0.93 & 0.95 & 0.92 & 0.89 & 0.90 & 0.90 & 0.90 & 0.82 & 0.84 & 0.90 & 0.81 & 0.52 & 0.69 & 0.56 \\
\hline 0.97 & 0.93 & 0.96 & 0.92 & 0.89 & 0.90 & 0.90 & 0.90 & 0.82 & 0.84 & 0.90 & 0.81 & 0.52 & 0.69 & 0.56 \\
\hline 0.97 & 0.93 & 0.96 & 0.92 & 0.89 & 0.90 & 0.90 & 0.90 & 0.82 & 0.84 & 0.90 & 0.81 & 0.52 & 0.69 & 0.56 \\
\hline 0.97 & 0.93 & 0.96 & 0.92 & 0.89 & 0.90 & 0.90 & 0.90 & 0.82 & 0.84 & 0.90 & 0.81 & 0.52 & 0.69 & 0.56 \\
\hline 0.58 & 0.35 & 0.57 & 0.37 & 0.33 & 0.62 & 0.49 & 0.50 & 0.40 & 0.78 & 0.68 & 0.74 & 0.73 & 0.62 & 0.37 \\
\hline 0.59 & 0.36 & 0.58 & 0.38 & 0.34 & 0.63 & 0.50 & 0.51 & 0.41 & 0.79 & 0.69 & 0.75 & 0.72 & 0.61 & 0.37 \\
\hline 0.61 & 0.39 & 0.60 & 0.40 & 0.36 & 0.64 & 0.51 & 0.53 & 0.43 & 0.80 & 0.70 & 0.76 & 0.72 & 0.62 & 0.38 \\
\hline 0.59 & 0.37 & 0.58 & 0.38 & 0.34 & 0.63 & 0.50 & 0.51 & 0.42 & 0.79 & 0.69 & 0.75 & 0.72 & 0.61 & 0.37 \\
\hline 0.59 & 0.36 & 0.58 & 0.38 & 0.34 & 0.62 & 0.49 & 0.51 & 0.41 & 0.78 & 0.68 & 0.75 & 0.72 & 0.61 & 0.37 \\
\hline 0.61 & 0.38 & 0.60 & 0.40 & 0.36 & 0.64 & 0.51 & 0.52 & 0.43 & 0.79 & 0.70 & 0.75 & 0.73 & 0.62 & 0.38 \\
\hline 0.60 & 0.37 & 0.59 & 0.38 & 0.34 & 0.63 & 0.50 & 0.51 & 0.42 & 0.79 & 0.69 & 0.75 & 0.72 & 0.62 & 0.37 \\
\hline 0.68 & 0.47 & 0.67 & 0.48 & 0.44 & 0.69 & 0.58 & 0.59 & 0.49 & 0.83 & 0.75 & 0.79 & 0.73 & 0.66 & 0.42 \\
\hline 0.68 & 0.47 & 0.67 & 0.48 & 0.44 & 0.70 & 0.58 & 0.59 & 0.49 & 0.83 & 0.75 & 0.79 & 0.73 & 0.66 & 0.42 \\
\hline 0.59 & 0.36 & 0.58 & 0.38 & 0.34 & 0.62 & 0.49 & 0.51 & 0.41 & 0.78 & 0.68 & 0.75 & 0.72 & 0.61 & 0.37 \\
\hline 0.60 & 0.37 & 0.59 & 0.39 & 0.35 & 0.63 & 0.50 & 0.51 & 0.42 & 0.79 & 0.69 & 0.75 & 0.72 & 0.62 & 0.37 \\
\hline 0.59 & 0.36 & 0.58 & 0.37 & 0.33 & 0.62 & 0.49 & 0.50 & 0.41 & 0.78 & 0.68 & 0.74 & 0.72 & 0.61 & 0.37 \\
\hline 0.58 & 0.35 & 0.56 & 0.36 & 0.32 & 0.62 & 0.48 & 0.49 & 0.40 & 0.78 & 0.67 & 0.74 & 0.72 & 0.60 & 0.36 \\
\hline 0.59 & 0.36 & 0.58 & 0.37 & 0.34 & 0.62 & 0.49 & 0.50 & 0.41 & 0.78 & 0.68 & 0.74 & 0.72 & 0.61 & 0.37 \\
\hline 0.61 & 0.38 & 0.59 & 0.40 & 0.36 & 0.64 & 0.51 & 0.52 & 0.43 & 0.79 & 0.70 & 0.76 & 0.72 & 0.62 & 0.38 \\
\hline 0.62 & 0.39 & 0.60 & 0.41 & 0.37 & 0.65 & 0.52 & 0.53 & 0.44 & 0.80 & 0.71 & 0.76 & 0.72 & 0.62 & 0.38 \\
\hline 0.66 & 0.45 & 0.65 & 0.46 & 0.43 & 0.69 & 0.57 & 0.58 & 0.48 & 0.83 & 0.74 & 0.79 & 0.71 & 0.64 & 0.40 \\
\hline 0.96 & 0.94 & 0.95 & 0.94 & 0.93 & 0.91 & 0.92 & 0.92 & 0.87 & 0.82 & 0.90 & 0.81 & 0.48 & 0.66 & 0.55 \\
\hline
\end{tabular}


Experiment

LEU-COMP-THERM-009-002 LEU-COMP-THERM-009-003 LEU-COMP-THERM-009-004 LEU-COMP-THERM-009-005 LEU-COMP-THERM-009-006 LEU-COMP-THERM-009-007 LEU-COMP-THERM-009-008 LEU-COMP-THERM-009-009 LEU-COMP-THERM-009-010 LEU-COMP-THERM-009-011 LEU-COMP-THERM-009-012 LEU-COMP-THERM-009-013 LEU-COMP-THERM-009-014 LEU-COMP-THERM-009-015 LEU-COMP-THERM-009-016 LEU-COMP-THERM-009-017 LEU-COMP-THERM-009-018 LEU-COMP-THERM-009-019 LEU-COMP-THERM-009-020 LEU-COMP-THERM-009-021 LEU-COMP-THERM-009-022 LEU-COMP-THERM-009-023 LEU-COMP-THERM-009-024 LEU-COMP-THERM-009-025 LEU-COMP-THERM-009-026 LEU-COMP-THERM-009-027 LEU-COMP-THERM-010-001 LEU-COMP-THERM-010-002 LEU-COMP-THERM-010-003 LEU-COMP-THERM-010-004 LEU-COMP-THERM-010-005 LEU-COMP-THERM-010-006 LEU-COMP-THERM-010-007 LEU-COMP-THERM-010-008 LEU-COMP-THERM-010-009 LEU-COMP-THERM-010-010 LEU-COMP-THERM-010-011_ LEU-COMP-THERM-010-012 LEU-COMP-THERM-010-013 LEU-COMP-THERM-010-014 LEU-COMP-THERM-010-015 LEU-COMP-THERM-010-016_-

\begin{tabular}{|c|c|c|c|c|c|c|c|c|c|c|c|c|c|c|}
\hline 1 & 2 & 3 & 4 & 5 & 6 & 7 & 8 & 9 & 10 & 11 & 12 & 13 & 14 & 15 \\
\hline 0.96 & 0.95 & 0.95 & 0.94 & 0.93 & 0.90 & 0.92 & 0.91 & 0.86 & 0.81 & 0.89 & 0.80 & 0.47 & 0.65 & 5 \\
\hline 0.96 & 0.95 & 0.95 & 0.94 & 0.93 & 0.90 & 0.92 & 0.91 & 0.86 & 0.81 & 0.89 & 0.80 & 0.47 & 0.65 & \\
\hline 0.96 & 0.94 & 0.95 & 0.94 & 0.93 & 0.89 & 0.91 & 0.91 & 0.86 & 0.81 & 0.89 & 0.80 & 0.47 & 0.65 & \\
\hline 0.96 & 0.94 & 0.95 & 0.93 & 0.93 & 0.90 & 0.92 & 0.91 & 0.86 & 0.82 & 0.90 & 0.80 & 0.48 & 0.66 & \\
\hline 0.96 & 0.95 & 0.95 & 0.94 & 0.94 & 0.89 & 0.92 & 0.91 & 0.86 & 0.80 & 0.89 & 0.79 & 0.45 & 0.65 & \\
\hline 0.96 & 0.96 & 0.95 & 0.94 & 0.94 & 0.89 & 0.92 & 0.91 & 0.86 & 0.80 & 0.89 & 0.79 & 0.46 & 0.65 & \\
\hline 0.96 & 0.95 & 0.95 & 0.94 & 0.93 & 0.89 & 0.91 & 0.91 & 0.86 & 0.81 & 0.89 & 0.80 & 0.47 & 0.65 & \\
\hline 0.96 & 0.94 & 0.95 & 0.93 & 0.93 & 0.90 & 0.91 & 0.91 & 0.86 & 0.82 & 0.90 & 0.80 & 0.47 & 0.65 & \\
\hline 0.96 & 0.95 & 0.95 & 0.94 & 0.93 & 0.89 & 0.91 & 0.91 & 0.86 & 0.81 & 0.89 & 0.79 & 0.47 & 0.65 & \\
\hline 0.96 & 0.95 & 0.95 & 0.94 & 0.93 & 0.89 & 0.92 & 0.91 & 0.86 & 0.80 & 0.89 & 0.79 & 0.46 & 0.65 & \\
\hline 0.96 & 0.95 & 0.95 & 0.94 & 0.93 & 0.89 & 0.91 & 0.91 & 0.86 & 0.80 & 0.89 & 0.79 & 0.46 & 0.65 & \\
\hline 0.96 & 0.94 & 0.95 & 0.93 & 0.93 & 0.89 & 0.91 & 0.91 & 0.85 & 0.81 & 0.89 & 0.80 & 0.47 & 0.65 & \\
\hline 0.96 & 0.95 & 0.95 & 0.94 & 0.93 & 0.89 & 0.92 & 0.91 & 0.86 & 0.80 & 0.89 & 0.79 & 0.46 & 0.65 & \\
\hline 0.96 & 0.94 & 0.95 & 0.93 & 0.93 & 0.90 & 0.91 & 0.91 & 0.86 & 0.81 & 0.89 & 0.80 & 0.47 & 0.65 & \\
\hline 0.96 & 0.95 & 0.95 & 0.94 & 0.93 & 0.90 & 0.92 & 0.91 & 0.86 & 0.81 & 0.89 & 0.80 & 0.47 & 0.65 & \\
\hline 0.96 & 0.95 & 0.95 & 0.94 & 0.93 & 0.89 & 0.91 & 0.91 & 0.86 & 0.81 & 0.89 & 0.79 & 0.46 & 0.65 & \\
\hline 0.96 & 0.95 & 0.95 & 0.94 & 0.93 & 0.90 & 0.92 & 0.91 & 0.86 & 0.81 & 0.89 & 0.79 & 0.47 & 0.65 & \\
\hline 0.96 & 0.95 & 0.95 & 0.94 & 0.93 & 0.89 & 0.92 & 0.91 & 0.86 & 0.81 & 0.89 & 0.79 & 0.47 & 0.65 & \\
\hline 0.96 & 0.94 & 0.95 & 0.93 & 0.93 & 0.90 & 0.91 & 0.91 & 0.86 & 0.82 & 0.90 & 0.80 & 0.47 & 0.65 & \\
\hline 0.96 & 0.95 & 0.95 & 0.94 & 0.93 & 0.89 & 0.92 & 0.91 & 0.86 & 0.81 & 0.89 & 0.80 & 0.47 & 0.65 & \\
\hline 0.96 & 0.94 & 0.95 & 0.93 & 0.93 & 0.90 & 0.91 & 0.91 & 0.86 & 0.81 & 0.89 & 0.80 & 0.47 & 0.65 & \\
\hline 0.96 & 0.95 & 0.95 & 0.94 & 0.94 & 0.89 & 0.92 & 0.91 & 0.86 & 0.80 & 0.89 & 0.79 & 0.46 & 0.65 & \\
\hline 0.96 & 0.94 & 0.95 & 0.93 & 0.93 & 0.89 & 0.91 & 0.91 & 0.86 & 0.81 & 0.89 & 0.80 & 0.47 & 0.65 & \\
\hline 0.96 & 0.95 & 0.95 & 0.94 & 0.93 & 0.89 & 0.91 & 0.91 & 0.86 & 0.80 & 0.89 & 0.79 & 0.46 & 0.65 & \\
\hline 0.96 & 0.95 & 0.95 & 0.94 & 0.94 & 0.89 & 0.92 & 0.91 & 0.86 & 0.80 & 0.89 & 0.79 & 0.46 & 0.65 & \\
\hline 0.96 & 0.95 & 0.95 & 0.94 & 0.93 & 0.89 & 0.91 & 0.91 & 0.86 & 0.81 & 0.89 & 0.79 & 0.46 & 0.65 & \\
\hline 0.95 & 0.93 & 0.94 & 0.92 & 0.91 & 0.89 & 0.90 & 0.90 & 0.84 & 0.81 & 0.89 & 0.80 & 0.48 & 0.66 & \\
\hline 0.96 & 0.93 & 0.95 & 0.93 & 0.92 & 0.89 & 0.91 & 0.90 & 0.85 & 0.82 & 0.89 & 0.80 & 0.48 & 0.66 & \\
\hline 0.95 & 0.93 & 0.95 & 0.92 & 0.92 & 0.89 & 0.91 & 0.90 & 0.85 & 0.82 & 0.90 & 0.80 & 0.48 & 0.66 & \\
\hline 0.96 & 0.93 & 0.95 & 0.93 & 0.92 & 0.90 & 0.91 & 0.90 & 0.85 & 0.82 & 0.90 & 0.81 & 0.48 & 0.65 & \\
\hline 0.92 & 0.81 & 0.90 & 0.81 & 0.79 & 0.88 & 0.84 & 0.84 & 0.77 & 0.89 & 0.90 & 0.86 & 0.64 & 0.71 & \\
\hline 0.94 & 0.87 & & & & 0.90 & 0.88 & 0.88 & 0.81 & 0.87 & 0.91 & 0.85 & & 0.69 & \\
\hline 0.95 & 0.90 & 0.94 & 0.90 & 0.89 & 0.90 & 0.90 & 0.89 & 0.84 & 0.85 & 0.90 & 0.83 & 0.54 & 0.67 & \\
\hline 0.95 & 0.90 & 0.93 & 0.90 & 0.89 & 0.90 & 0.90 & 0.89 & 0.84 & 0.84 & 0.90 & 0.82 & 0.52 & 0.67 & \\
\hline 0.92 & 0.87 & 0.91 & 0.91 & 0.90 & 0.93 & 0.93 & 0.93 & 0.88 & 0.89 & 0.93 & 0.87 & 0.55 & 0.70 & \\
\hline 0.94 & 0.89 & 0.93 & 0.92 & 0.91 & 0.93 & 0.94 & 0.94 & 0.89 & 0.88 & 0.94 & 0.86 & 0.54 & 0.69 & \\
\hline 0.95 & 0.90 & 0.94 & 0.93 & 0.92 & 0.93 & 0.93 & 0.93 & 0.88 & 0.87 & 0.93 & 0.86 & 0.53 & 0.69 & \\
\hline 0.96 & 0.92 & 0.95 & 0.93 & 0.92 & 0.92 & 0.93 & 0.92 & 0.87 & 0.86 & 0.92 & 0.84 & 0.51 & 0.68 & \\
\hline 0.96 & 0.92 & 0.95 & 0.92 & 0.91 & 0.91 & 0.91 & 0.91 & 0.85 & 0.84 & 0.91 & 0.82 & 0.50 & 0.66 & \\
\hline 0.97 & 0.90 & 0.96 & 0.91 & 0.88 & 0.91 & 0.90 & 0.93 & 0.83 & 0.88 & 0.93 & 0.87 & 0.60 & 0.76 & \\
\hline 0.98 & 0.91 & 0.97 & 0.91 & 0.89 & 0.91 & 0.91 & 0.93 & 0.84 & 0.88 & 0.93 & 0.86 & 0.59 & 0.76 & \\
\hline 0.97 & 0.90 & 0.97 & 0.90 & 0.88 & 0.91 & 0.90 & 0.92 & 0.83 & 0.88 & 0.93 & 0.87 & 0.60 & 0.76 & \\
\hline
\end{tabular}


Experiment

LEU-COMP-THERM-010-017 LEU-COMP-THERM-010-018 LEU-COMP-THERM-010-019 LEU-COMP-THERM-010-020 LEU-COMP-THERM-010-021 LEU-COMP-THERM-010-022 LEU-COMP-THERM-010-023 LEU-COMP-THERM-010-024 LEU-COMP-THERM-010-025 LEU-COMP-THERM-010-026 LEU-COMP-THERM-010-027 LEU-COMP-THERM-010-028 LEU-COMP-THERM-010-029 LEU-COMP-THERM-010-030 LEU-COMP-THERM-011-001 LEU-COMP-THERM-011-002 LEU-COMP-THERM-011-003 LEU-COMP-THERM-011-004 LEU-COMP-THERM-011-005 LEU-COMP-THERM-011-006 LEU-COMP-THERM-011-007 LEU-COMP-THERM-011-008 LEU-COMP-THERM-011-009 LEU-COMP-THERM-011-010 LEU-COMP-THERM-011-011 LEU-COMP-THERM-011-012 LEU-COMP-THERM-011-013 LEU-COMP-THERM-011-014 LEU-COMP-THERM-011-015 LEU-COMP-THERM-012-001 LEU-COMP-THERM-012-002 LEU-COMP-THERM-012-003 LEU-COMP-THERM-012-004 LEU-COMP-THERM-012-005 LEU-COMP-THERM-012-006 LEU-COMP-THERM-012-007 LEU-COMP-THERM-012-008 LEU-COMP-THERM-012-009 LEU-COMP-THERM-012-010 LEU-COMP-THERM-013-001_ LEU-COMP-THERM-013-002 LEU-COMP-THERM-013-003

\begin{tabular}{|c|c|c|c|c|c|c|c|c|c|c|c|c|c|c|}
\hline 1 & 2 & 3 & 4 & 5 & 6 & 7 & 8 & 9 & 10 & 11 & 12 & 13 & 14 & 15 \\
\hline 0.98 & 0.92 & 0.98 & 0.92 & 0.89 & 0.91 & 0.90 & 0.93 & 0.83 & 0.87 & 0.93 & 0.85 & 0.58 & 0.75 & 0.62 \\
\hline 0.99 & 0.93 & 0.98 & 0.92 & 0.90 & 0.90 & 0.90 & 0.93 & 0.83 & 0.86 & 0.92 & 0.84 & 0.57 & 0.74 & 0.62 \\
\hline 0.99 & 0.93 & 0.98 & 0.91 & 0.89 & 0.89 & 0.89 & 0.91 & 0.82 & 0.85 & 0.91 & 0.83 & 0.56 & 0.73 & 0.62 \\
\hline 0.98 & 0.92 & 0.97 & 0.90 & 0.88 & 0.88 & 0.88 & 0.90 & 0.80 & 0.83 & 0.90 & 0.82 & 0.55 & 0.73 & 0.62 \\
\hline 0.99 & 0.94 & 0.98 & 0.92 & 0.89 & 0.88 & 0.89 & 0.91 & 0.81 & 0.83 & 0.90 & 0.81 & 0.54 & 0.73 & 0.62 \\
\hline 0.99 & 0.94 & 0.98 & 0.92 & 0.90 & 0.88 & 0.89 & 0.91 & 0.81 & 0.83 & 0.90 & 0.81 & 0.53 & 0.72 & 0.62 \\
\hline 0.99 & 0.94 & 0.98 & 0.92 & 0.89 & 0.89 & 0.89 & 0.91 & 0.81 & 0.84 & 0.91 & 0.82 & 0.54 & 0.73 & 0.62 \\
\hline 0.95 & 0.84 & 0.94 & 0.83 & 0.79 & 0.87 & 0.84 & 0.86 & 0.75 & 0.88 & 0.91 & 0.86 & 0.66 & 0.77 & 0.61 \\
\hline 0.97 & 0.88 & 0.96 & 0.86 & 0.83 & 0.88 & 0.86 & 0.88 & 0.77 & 0.87 & 0.91 & 0.85 & 0.63 & 0.76 & 0.62 \\
\hline 0.97 & 0.89 & 0.96 & 0.87 & 0.85 & 0.89 & 0.87 & 0.89 & 0.79 & 0.87 & 0.91 & 0.85 & 0.62 & 0.76 & 0.62 \\
\hline 0.98 & 0.90 & 0.97 & 0.88 & 0.86 & 0.89 & 0.87 & 0.90 & 0.79 & 0.86 & 0.91 & 0.84 & 0.60 & 0.75 & 0.62 \\
\hline 0.98 & 0.91 & 0.97 & 0.89 & 0.87 & 0.89 & 0.88 & 0.90 & 0.80 & 0.86 & 0.91 & 0.84 & 0.59 & 0.75 & 0.62 \\
\hline 0.99 & 0.92 & 0.98 & 0.90 & 0.88 & 0.89 & 0.89 & 0.90 & 0.81 & 0.85 & 0.91 & 0.83 & 0.58 & 0.74 & 0.62 \\
\hline 0.99 & 0.93 & 0.98 & 0.91 & 0.88 & 0.89 & 0.89 & 0.91 & 0.81 & 0.85 & 0.91 & 0.83 & 0.57 & 0.74 & 0.62 \\
\hline 0.97 & 0.91 & 0.96 & 0.89 & 0.86 & 0.89 & 0.88 & 0.89 & 0.79 & 0.85 & 0.90 & 0.82 & 0.57 & 0.72 & .58 \\
\hline 0.80 & 0.62 & 0.79 & 0.62 & 0.58 & 0.78 & 0.69 & 0.71 & 0.59 & 0.87 & 0.83 & 0.84 & 0.72 & 0.72 & 0.50 \\
\hline 0.80 & 0.62 & 0.78 & 0.62 & 0.58 & 0.79 & 0.70 & 0.71 & 0.60 & 0.88 & 0.83 & 0.84 & 0.71 & 0.70 & 0.48 \\
\hline 0.80 & 0.61 & 0.78 & 0.62 & 0.58 & 0.79 & 0.69 & 0.71 & 0.60 & 0.88 & 0.83 & 0.84 & 0.71 & 0.70 & 0.48 \\
\hline 0.80 & 0.62 & 0.78 & 0.62 & 0.58 & 0.79 & 0.69 & 0.71 & 0.60 & 0.88 & 0.83 & 0.84 & 0.71 & 0.70 & .48 \\
\hline 0.80 & 0.62 & 0.79 & 0.63 & 0.59 & 0.79 & 0.70 & 0.71 & 0.61 & 0.88 & 0.84 & 0.84 & 0.71 & 0.70 & 0.48 \\
\hline 0.81 & 0.64 & 0.80 & 0.64 & 0.60 & 0.80 & 0.71 & 0.72 & 0.62 & 0.88 & 0.84 & 0.85 & 0.70 & 0.71 & 0.49 \\
\hline 0.82 & 0.65 & 0.81 & 0.65 & 0.61 & 0.80 & 0.72 & 0.73 & 0.62 & 0.88 & 0.85 & 0.85 & 0.70 & 0.71 & 0.50 \\
\hline 0.83 & 0.66 & 0.81 & 0.66 & 0.62 & 0.81 & 0.72 & 0.74 & 0.63 & 0.89 & 0.85 & 0.85 & 0.70 & 0.71 & 0.50 \\
\hline 0.81 & 0.64 & 0.80 & .64 & 0.60 & 0.80 & 0.71 & 0.72 & 0.61 & 0.88 & 0.84 & 0.84 & 0.71 & 0.71 & 0.49 \\
\hline 0.83 & 0.66 & 0.81 & 0.66 & 0.63 & 0.81 & 0.72 & 0.74 & 0.63 & 0.88 & 0.85 & 0.84 & 0.69 & 0.71 & 0.49 \\
\hline 0.84 & 0.68 & 0.83 & 0.68 & 0.65 & 0.82 & 0.74 & 0.75 & 0.65 & 0.89 & 0.86 & 0.85 & 0.69 & 0.71 & 0.50 \\
\hline 0.83 & 0.67 & 0.81 & 0.68 & 0.64 & 0.81 & 0.73 & 0.74 & 0.64 & 0.88 & 0.85 & 0.84 & 0.67 & 0.69 & 0.49 \\
\hline 0.85 & 0.70 & 0.83 & 0.70 & 0.67 & 0.83 & 0.75 & 0.76 & 0.66 & 0.88 & 0.86 & 0.85 & 0.67 & 0.70 & 0.50 \\
\hline 0.84 & 0.68 & 0.81 & 0.70 & 0.66 & 0.81 & 0.73 & 0.75 & 0.65 & 0.87 & 0.85 & 0.83 & 0.64 & 0.68 & 0.49 \\
\hline 0.95 & 0.85 & 0.93 & 0.85 & 0.81 & 0.89 & 0.86 & 0.88 & 0.77 & 0.88 & 0.91 & 0.86 & 0.62 & 0.74 & 0.58 \\
\hline 0.94 & 0.83 & 0.92 & 0.82 & 0.78 & 0.87 & 0.83 & 0.85 & 0.74 & 0.88 & 0.90 & 0.85 & 0.63 & 0.74 & 0.57 \\
\hline 0.95 & 0.85 & 0.93 & 0.84 & 0.80 & 0.88 & 0.84 & 0.86 & 0.75 & 0.88 & 0.90 & 0.85 & 0.63 & 0.74 & 0.57 \\
\hline 0.95 & 0.84 & 0.93 & 0.83 & 0.79 & 0.87 & 0.83 & 0.85 & 0.74 & 0.88 & 0.90 & 0.85 & 0.63 & 0.74 & 0.57 \\
\hline 0.94 & 0.84 & 0.93 & 0.82 & 0.79 & 0.88 & 0.84 & 0.85 & 0.74 & 0.88 & 0.90 & 0.85 & 0.63 & 0.74 & 0.57 \\
\hline 0.94 & 0.84 & 0.93 & 0.83 & 0.79 & 0.88 & 0.84 & 0.86 & 0.74 & 0.88 & 0.90 & 0.85 & 0.63 & 0.74 & 0.57 \\
\hline 0.94 & 0.83 & 0.92 & 0.82 & 0.78 & 0.87 & 0.83 & 0.85 & 0.74 & 0.88 & 0.90 & 0.85 & 0.64 & 0.74 & 0.57 \\
\hline 0.94 & 0.84 & 0.93 & 0.83 & 0.79 & 0.87 & 0.83 & 0.85 & 0.74 & 0.88 & 0.90 & 0.85 & 0.63 & 0.74 & 0.57 \\
\hline 0.94 & 0.84 & 0.92 & 0.83 & 0.79 & 0.87 & 0.84 & 0.85 & 0.74 & 0.87 & 0.90 & 0.85 & 0.62 & 0.73 & 0.5 \\
\hline 0.94 & 0.83 & 0.92 & 0.83 & 0.79 & 0.88 & 0.84 & 0.85 & 0.74 & 0.88 & 0.90 & 0.85 & 0.63 & 0.74 & 0.57 \\
\hline 0.98 & 0.91 & 0.97 & 0.92 & 0.89 & 0.92 & 0.91 & 0.94 & 0.84 & 0.89 & 0.94 & 0.87 & 0.60 & 0.76 & 0.63 \\
\hline 0.98 & 0.92 & 0.98 & 0.92 & 0.89 & 0.91 & 0.91 & 0.93 & 0.84 & 0.87 & 0.93 & 0.86 & 0.59 & 0.76 & 0.63 \\
\hline 0.98 & 0.91 & 0.97 & 0.91 & 0.88 & 0.91 & 0.90 & 0.93 & 0.83 & 0.88 & 0.94 & 0.87 & 0.60 & 0.76 & 66 \\
\hline
\end{tabular}


Experiment

LEU-COMP-THERM-013-004 LEU-COMP-THERM-013-005 LEU-COMP-THERM-013-006 LEU-COMP-THERM-013-007 LEU-COMP-THERM-014-001 LEU-COMP-THERM-014-002 LEU-COMP-THERM-014-005 LEU-COMP-THERM-014-006 LEU-COMP-THERM-014-007 LEU-COMP-THERM-015-001 LEU-COMP-THERM-015-002 LEU-COMP-THERM-015-003 LEU-COMP-THERM-015-004 LEU-COMP-THERM-015-005 LEU-COMP-THERM-015-006 LEU-COMP-THERM-015-007 LEU-COMP-THERM-015-008 LEU-COMP-THERM-015-009 LEU-COMP-THERM-015-010 LEU-COMP-THERM-015-011 LEU-COMP-THERM-015-012 LEU-COMP-THERM-015-013 LEU-COMP-THERM-015-014 LEU-COMP-THERM-015-015 LEU-COMP-THERM-015-016 LEU-COMP-THERM-015-017 LEU-COMP-THERM-015-018 LEU-COMP-THERM-015-019 LEU-COMP-THERM-015-020 LEU-COMP-THERM-015-021 LEU-COMP-THERM-015-022 LEU-COMP-THERM-015-023 LEU-COMP-THERM-015-024 LEU-COMP-THERM-015-025 LEU-COMP-THERM-015-026 LEU-COMP-THERM-015-027 LEU-COMP-THERM-015-028 LEU-COMP-THERM-015-029 LEU-COMP-THERM-015-030 LEU-COMP-THERM-015-031 LEU-COMP-THERM-015-032 LEU-COMP-THERM-015-033

\begin{tabular}{|c|c|c|c|c|c|c|c|c|c|c|c|c|c|c|}
\hline 1 & 2 & 3 & 4 & 5 & 6 & 7 & 8 & 9 & 10 & 11 & 12 & 13 & 14 & 15 \\
\hline 0.98 & 0.91 & 0.97 & 0.91 & 0.88 & 0.91 & 0.91 & 0.93 & 0.83 & 0.88 & 0.94 & 0.87 & 0.60 & 0.76 & 62 \\
\hline 0.98 & 0.92 & 0.98 & 0.92 & 0.89 & 0.91 & 0.91 & 0.93 & 0.84 & 0.87 & 0.93 & 0.86 & 0.59 & 0.76 & \\
\hline 0.98 & 0.92 & 0.97 & 0.92 & 0.89 & 0.91 & 0.91 & 0.93 & 0.84 & 0.87 & 0.93 & 0.85 & 0.58 & 0.75 & \\
\hline 0.98 & 0.92 & 0.97 & 0.91 & 0.89 & 0.91 & 0.91 & 0.93 & 0.83 & 0.88 & 0.93 & 0.86 & 0.59 & 0.76 & \\
\hline 0.99 & 0.96 & 0.98 & 0.93 & 0.91 & 0.89 & 0.91 & 0.92 & 0.83 & 0.83 & 0.91 & 0.81 & 0.53 & 0.72 & \\
\hline 0.99 & 0.94 & 0.99 & 0.91 & 0.89 & 0.89 & 0.89 & 0.91 & 0.82 & 0.84 & 0.91 & 0.83 & 0.56 & 0.74 & \\
\hline 0.82 & 0.64 & 0.82 & 0.63 & 0.60 & 0.78 & 0.70 & 0.73 & 0.62 & 0.88 & 0.85 & 0.86 & 0.75 & 0.76 & \\
\hline 0.98 & 0.91 & 0.97 & 0.88 & 0.85 & 0.87 & 0.86 & 0.89 & 0.78 & 0.84 & 0.90 & 0.83 & 0.60 & 0.77 & \\
\hline 0.94 & 0.83 & 0.93 & 0.80 & 0.76 & 0.84 & 0.81 & 0.84 & 0.72 & 0.85 & 0.88 & 0.84 & 0.67 & 0.79 & \\
\hline 0.99 & 0.93 & 0.98 & 0.92 & 0.89 & 0.90 & 0.90 & 0.92 & 0.82 & 0.86 & 0.92 & 0.84 & 0.57 & 0.74 & \\
\hline 0.99 & 0.93 & 0.98 & 0.91 & 0.89 & 0.89 & 0.89 & 0.91 & 0.81 & 0.85 & 0.91 & 0.83 & 0.56 & 0.73 & \\
\hline 0.99 & 0.94 & 0.98 & 0.92 & 0.90 & 0.89 & 0.90 & 0.91 & 0.82 & 0.84 & 0.91 & 0.82 & 0.55 & 0.73 & \\
\hline 0.99 & 0.94 & 0.98 & 0.92 & 0.89 & 0.89 & 0.90 & 0.91 & 0.81 & 0.84 & 0.91 & 0.82 & 0.56 & 0.73 & \\
\hline 0.99 & 0.94 & 0.98 & 0.92 & 0.89 & 0.89 & 0.90 & 0.91 & 0.81 & 0.84 & .91 & 0.82 & 0.55 & 0.73 & \\
\hline 0.99 & 0.94 & 0.98 & 0.92 & 0.89 & 0.89 & 0.90 & 0.91 & 0.82 & 0.84 & 0.91 & 0.82 & 0.55 & 0.73 & \\
\hline 0.99 & 0.94 & 0.98 & 0.92 & 0.89 & 0.89 & 0.90 & 0.91 & 0.81 & 0.84 & 0.91 & 0.82 & 0.55 & 0.73 & \\
\hline 0.99 & 0.94 & 0.98 & 0.92 & 0.89 & 0.89 & 0.90 & 0.91 & 0.81 & 0.84 & 0.91 & 0.82 & 0.55 & 0.73 & \\
\hline 0.99 & 0.94 & 0.98 & 0.92 & 0.89 & 0.89 & 0.90 & 0.91 & 0.81 & 0.84 & 91 & 0.82 & 0.56 & 0.74 & \\
\hline 0.99 & 0.93 & 0.98 & 0.91 & 0.89 & 0.89 & 0.89 & 0.91 & 0.81 & 0.84 & .91 & 0.82 & 0.56 & 0.74 & \\
\hline 0.99 & 0.94 & 0.98 & 0.91 & 0.89 & 0.89 & 0.89 & 0.91 & 0.81 & 0.84 & 0.91 & 0.82 & 0.56 & 0.74 & \\
\hline 0.99 & 0.93 & 0.98 & 0.91 & 0.88 & 0.89 & 0.89 & 0.91 & 0.81 & 0.85 & 0.91 & 0.83 & 0.57 & 0.74 & \\
\hline 0.98 & 0.92 & 0.97 & 0.90 & 0.87 & 0.8 & 0.89 & 0.91 & 0.80 & 0.85 & 1 & 0.83 & 0. & 0.75 & \\
\hline 0.98 & 0.92 & 0.97 & 0.90 & 0.87 & 0.89 & 0.89 & 0.91 & 0.80 & 0.85 & 0.91 & 0.83 & 0.58 & 0.75 & \\
\hline 0.98 & 0.91 & 0.97 & 0.89 & 0.86 & 0.88 & 0.88 & 0.90 & 0.79 & 0.85 & 0.91 & 0.84 & 0.59 & 0.75 & \\
\hline 0.96 & 0.86 & 0.95 & 0.84 & 0.81 & 0.88 & 0.85 & 0.88 & 0.77 & 0.89 & 0.92 & 0.87 & 0.65 & 0.77 & \\
\hline 0.97 & 0.87 & 0.96 & 0.85 & 0.82 & 0.89 & 0.86 & 0.88 & 0.77 & 0.88 & 0.92 & 0.86 & 0.64 & 0.77 & \\
\hline 0.97 & 0.87 & 0.96 & 0.85 & 0.82 & 0.88 & 0.86 & 0.88 & 0.77 & 0.88 & 0.92 & 0.86 & 0.64 & 0.77 & \\
\hline 0.97 & 0.87 & 0.96 & 0.85 & 0.82 & 0.88 & 0.86 & 0.88 & 0.77 & 0.87 & 0.91 & 0.86 & 0.63 & 0.77 & \\
\hline 0.96 & 0.87 & 0.95 & 0.84 & 0.81 & 0.87 & 0.85 & 0.87 & 0.76 & 0.87 & 0.91 & 0.85 & 0.63 & 0.77 & \\
\hline 0.97 & 0.87 & 0.96 & 0.85 & 0.82 & 0.88 & 0.86 & 0.88 & 0.77 & 0.87 & 0.91 & 0.85 & 0.63 & 0.77 & \\
\hline 0.96 & 0.87 & 0.95 & 0.85 & 0.81 & 0.88 & 0.85 & 0.88 & 0.76 & 0.87 & 0.91 & 0.85 & 0.64 & 0.77 & \\
\hline 0.97 & 0.87 & 0.96 & 0.85 & 0.82 & 0.88 & 0.85 & 0.88 & 0.76 & 0.87 & 0.91 & 0.85 & 0.63 & 0.77 & \\
\hline 0.94 & 0.83 & 0.94 & 0.81 & 0.78 & 0.87 & 0.83 & 0.86 & 0.74 & 0.89 & 0.91 & 0.87 & 0.67 & 0.77 & \\
\hline 0.94 & 0.82 & 0.93 & 0.80 & 0.77 & 0.86 & 0.82 & 0.85 & 0.73 & 0.89 & 0.91 & 0.86 & 0.67 & 0.77 & \\
\hline 0.92 & 0.78 & 0.91 & 0.77 & 0.73 & 0.86 & 0.81 & 0.83 & 0.72 & 0.90 & 0.90 & 0.88 & 0.71 & 0.78 & \\
\hline 0.92 & 0.78 & 0.91 & 0.77 & 0.73 & 0.86 & 0.80 & 0.83 & 0.71 & 0.90 & 0.90 & 0.87 & 0.70 & 0.78 & \\
\hline 0.90 & 0.77 & 0.89 & 0.75 & 0.71 & 0.82 & 0.77 & 0.81 & 0.67 & 0.86 & 0.87 & 0.83 & 0.72 & 0.80 & \\
\hline 0.90 & 0.77 & 0.89 & 0.75 & 0.70 & 0.82 & 0.77 & 0.80 & 0.67 & 0.85 & 0.86 & 0.83 & 0.72 & 0.80 & \\
\hline 0.91 & 0.78 & 0.90 & 0.76 & 0.72 & 0.81 & 0.77 & 0.81 & 0.67 & 0.84 & 0.86 & 0.82 & 0.70 & 0.79 & \\
\hline 0.91 & 0.78 & 0.90 & 0.76 & 0.72 & 0.82 & 0.77 & 0.81 & 0.68 & 0.85 & 0.86 & 0.83 & 0.70 & 0.79 & \\
\hline 0.91 & 0.78 & 0.90 & 0.76 & 0.71 & 0.81 & 0.77 & 0.81 & 0.67 & 0.84 & 0.86 & 0.82 & 0.70 & 0.79 & \\
\hline 0.90 & 0.78 & 0.89 & 0.75 & 0.71 & 0.81 & 0.77 & 0.80 & 0.67 & 0.84 & 0.86 & 0.82 & 0.71 & 0.79 & \\
\hline
\end{tabular}


Experiment

LEU-COMP-THERM-015-034 LEU-COMP-THERM-015-035 LEU-COMP-THERM-015-036 LEU-COMP-THERM-015-037 LEU-COMP-THERM-015-038 LEU-COMP-THERM-015-039 LEU-COMP-THERM-015-040 LEU-COMP-THERM-015-041 LEU-COMP-THERM-015-042 LEU-COMP-THERM-015-043 LEU-COMP-THERM-015-044 LEU-COMP-THERM-015-045 LEU-COMP-THERM-015-046 LEU-COMP-THERM-015-047 LEU-COMP-THERM-015-048 LEU-COMP-THERM-015-049 LEU-COMP-THERM-015-050 LEU-COMP-THERM-015-051 LEU-COMP-THERM-015-052 LEU-COMP-THERM-015-053 LEU-COMP-THERM-015-054 LEU-COMP-THERM-015-055 LEU-COMP-THERM-015-056 LEU-COMP-THERM-015-057 LEU-COMP-THERM-015-058 LEU-COMP-THERM-015-059 LEU-COMP-THERM-015-060 LEU-COMP-THERM-015-061 LEU-COMP-THERM-015-062 LEU-COMP-THERM-015-063 LEU-COMP-THERM-015-064 LEU-COMP-THERM-015-065 LEU-COMP-THERM-015-066 LEU-COMP-THERM-015-067 LEU-COMP-THERM-015-068 LEU-COMP-THERM-015-069 LEU-COMP-THERM-015-070 LEU-COMP-THERM-015-071 LEU-COMP-THERM-015-072 LEU-COMP-THERM-015-073 LEU-COMP-THERM-015-074 LEU-COMP-THERM-015-075

\begin{tabular}{|c|c|c|c|c|c|c|c|c|c|c|c|c|c|}
\hline 1 & 2 & 3 & 4 & 5 & 6 & 7 & 8 & 9 & 10 & 11 & 12 & 13 & 14 \\
\hline 0.89 & 0.76 & 0.88 & 0.74 & 0.69 & 0.81 & 0.76 & 0.80 & 0.66 & 0.85 & 0.86 & 0.83 & 0.71 & 0.79 \\
\hline 0.90 & 0.77 & 0.89 & 0.75 & 0.70 & 0.81 & 0.76 & 0.80 & 0.66 & 0.84 & 0.86 & 0.82 & 0.70 & 0.79 \\
\hline 0.91 & 0.78 & 0.90 & 0.76 & 0.71 & 0.81 & 0.77 & 0.81 & 0.67 & 0.84 & 0.86 & 0.82 & 0.70 & 0.79 \\
\hline 0.88 & 0.74 & 0.87 & 0.72 & 0.67 & 0.79 & 0.74 & 0.78 & 0.64 & 0.84 & 0.84 & 0.82 & 0.72 & 0.79 \\
\hline 0.88 & 0.74 & 0.87 & 0.72 & 0.68 & 0.80 & 0.75 & 0.78 & 0.65 & 0.85 & 0.85 & 0.82 & 0.73 & 0.80 \\
\hline 0.98 & 0.96 & 0.97 & 0.95 & 0.93 & 0.90 & 0.92 & 0.92 & 0.86 & 0.82 & 0.90 & 0.80 & 0.49 & 0.67 \\
\hline 0.98 & 0.96 & 0.97 & 0.95 & 0.94 & 0.90 & 0.92 & 0.92 & 0.86 & 0.82 & 0.90 & 0.80 & 0.49 & 0.68 \\
\hline 0.98 & 0.96 & 0.97 & 0.95 & 0.94 & 0.90 & 0.92 & 0.92 & 0.86 & 0.82 & 0.90 & 0.80 & 0.49 & 0.68 \\
\hline 0.98 & 0.96 & 0.97 & 0.95 & 0.94 & 0.90 & 0.92 & 0.92 & 0.86 & 0.82 & 0.91 & 0.81 & 0.49 & 0.68 \\
\hline 0.98 & 0.96 & 0.97 & 0.95 & 0.94 & 0.90 & 0.92 & 0.93 & 0.86 & 0.82 & 0.91 & 0.81 & 0.50 & 0.69 \\
\hline 0.98 & 0.96 & 0.97 & 0.95 & 0.94 & 0.90 & 0.93 & 0.93 & 0.86 & 0.82 & 0.91 & 0.81 & 0.50 & .69 \\
\hline 0.98 & 0.96 & 0.97 & 0.95 & 0.93 & 0.91 & 0.93 & 0.93 & 0.86 & 0.83 & 0.91 & 0.81 & 0.50 & 0.69 \\
\hline 0.98 & 0.96 & 0.97 & 0.95 & 0.93 & 0.91 & 0.93 & 0.93 & 0.86 & 0.83 & 0.91 & 0.81 & 0.50 & 0.69 \\
\hline 0.99 & 0.96 & 0.97 & 0.95 & 0.93 & 0.91 & 0.93 & 0.93 & 0.86 & 0.83 & 0.91 & 0.81 & 0.50 & 0.69 \\
\hline 0.97 & 0.89 & 0.96 & 0.87 & 0.85 & 0.91 & 0.89 & 0.89 & 0.81 & 0.89 & 0.93 & 0.87 & .60 & 0.73 \\
\hline 0.97 & 0.90 & 0.96 & 0.88 & 0.86 & 0.91 & 0.89 & 0.90 & 0.81 & 0.89 & 0.93 & 0.87 & 0.59 & 0.73 \\
\hline 0.97 & 0.89 & 0.96 & 0.88 & 0.85 & 0.91 & 0.89 & 0.90 & 0.81 & 0.89 & 0.93 & 0.87 & 0.60 & 0.73 \\
\hline 0.97 & 0.89 & 0.96 & 0.87 & 0.85 & 0.91 & 0.89 & 0.89 & 0.81 & 0.89 & 0.93 & 0.87 & 0.60 & 0.73 \\
\hline 0.97 & 0.89 & 0.96 & 0.88 & 0.85 & 0.91 & 0.89 & 0.90 & 0.81 & 0.89 & 0.93 & 0.87 & 0.60 & 0.73 \\
\hline 0.97 & 0.89 & 0.96 & 0.88 & 0.85 & 0.91 & 0.89 & 0.90 & 0.81 & 0.89 & 0.93 & 0.87 & 0.60 & 0.73 \\
\hline 0.97 & 0.97 & 0.97 & 0.96 & 0.95 & 0.89 & 0.93 & 0.92 & 0.87 & 0.80 & 0.89 & 0.79 & 0.46 & 0.66 \\
\hline 0.97 & 0.97 & 0.97 & 0.96 & 0.95 & 0.89 & 0.93 & 0.92 & 0.87 & 0.80 & 0.89 & 0.78 & 0.46 & 0.66 \\
\hline 0.98 & 0.98 & 0.97 & 0.96 & 0.95 & 0.90 & 0.93 & 0.93 & 0.87 & 0.80 & 0.89 & 0.79 & 0.46 & 0.67 \\
\hline 0.98 & 0.98 & 0.97 & 0.96 & 0.95 & 0.90 & 0.93 & 0.93 & 0.87 & 0.80 & 0.89 & 0.79 & 0.47 & 0.67 \\
\hline 0.98 & 0.98 & 0.97 & 0.96 & 0.95 & 0.90 & 0.93 & 0.93 & 0.87 & 0.80 & 0.90 & 0.79 & 0.47 & 0.67 \\
\hline 0.98 & 0.98 & 0.97 & 0.96 & 0.95 & 0.90 & 0.93 & 0.93 & 0.87 & 0.80 & 0.90 & 0.79 & 0.47 & 0.68 \\
\hline 0.93 & 0.91 & 0.92 & 0.91 & 0.91 & 0.89 & 0.90 & 0.89 & 0.85 & 0.81 & 0.88 & 0.79 & 0.47 & 0.63 \\
\hline 0.93 & 0.91 & 0.92 & 0.91 & 0.91 & 0.89 & 0.90 & 0.8 & 0.85 & 0.81 & 0.89 & 0.80 & 0.47 & 0.63 \\
\hline 0.94 & 0.92 & 0.93 & 0.92 & 0.91 & 0.89 & 0.91 & 0.89 & 0.85 & 0.82 & 0.89 & 0.80 & 0.47 & 0.63 \\
\hline 0.94 & 0.92 & 0.93 & 0.92 & 0.91 & 0.89 & 0.91 & 0.89 & 0.85 & 0.82 & 0.89 & 0.80 & 0.48 & 0.64 \\
\hline 0.94 & 0.92 & 0.93 & 0.92 & 0.91 & 0.90 & 0.91 & 0.90 & 0.85 & 0.82 & 0.89 & 0.80 & 0.47 & 0.64 \\
\hline 0.94 & 0.92 & 0.93 & 0.92 & 0.91 & 0.90 & 0.91 & 0.90 & 0.85 & 0.82 & 0.90 & 0.81 & 0.48 & 0.64 \\
\hline 0.94 & 0.92 & 0.93 & 0.92 & 0.91 & 0.90 & 0.91 & 0.90 & 0.85 & 0.83 & 0.90 & 0.81 & 0.48 & 0.64 \\
\hline 0.94 & 0.92 & 0.93 & 0.92 & 0.91 & 0.90 & 0.91 & 0.90 & 0.85 & 0.83 & 0.90 & 0.81 & 0.48 & 0.64 \\
\hline 0.94 & 0.92 & 0.93 & 0.92 & 0.91 & 0.90 & 0.91 & 0.90 & 0.85 & 0.83 & 0.90 & 0.81 & 0.48 & 0.65 \\
\hline 0.94 & 0.92 & 0.93 & 0.92 & 0.91 & 0.90 & 0.91 & 0.90 & 0.86 & 0.83 & 0.90 & 0.81 & 0.48 & 0.65 \\
\hline 0.93 & 0.82 & 0.91 & 0.81 & 0.77 & 0.87 & 0.82 & 0.84 & 0.72 & 0.87 & 0.89 & 0.84 & 0.65 & 0.74 \\
\hline 0.93 & 0.81 & 0.91 & 0.80 & 0.76 & 0.87 & 0.82 & 0.84 & 0.72 & 0.88 & 0.89 & 0.84 & 0.65 & 0.74 \\
\hline 0.92 & 0.79 & 0.90 & 0.78 & 0.74 & 0.86 & 0.80 & 0.82 & 0.71 & 0.88 & 0.89 & 0.85 & 0.68 & 0.75 \\
\hline 0.99 & 0.96 & 0.99 & 0.93 & 0.91 & 0.89 & 0.91 & 0.92 & 0.83 & 0.82 & 0.90 & 0.81 & 0.53 & 0.72 \\
\hline 0.99 & 0.96 & 0.99 & 0.94 & 0.92 & 0.90 & 0.91 & 0.93 & 0.84 & 0.84 & 0.91 & 0.82 & 0.54 & 0.73 \\
\hline 0.99 & 0.96 & 0.99 & 0.93 & 0.91 & 0.89 & 0.91 & 0.92 & 0.83 & 0.83 & 0.91 & 0.82 & 0.54 & 0.73 \\
\hline
\end{tabular}


Experiment

LEU-COMP-THERM-015-076 LEU-COMP-THERM-015-077 LEU-COMP-THERM-015-078 LEU-COMP-THERM-015-079 LEU-COMP-THERM-015-080 LEU-COMP-THERM-015-081 LEU-COMP-THERM-015-082 LEU-COMP-THERM-015-083 LEU-COMP-THERM-015-084 LEU-COMP-THERM-015-085 LEU-COMP-THERM-015-086 LEU-COMP-THERM-015-087 LEU-COMP-THERM-015-088 LEU-COMP-THERM-015-089 LEU-COMP-THERM-015-090 LEU-COMP-THERM-015-091 LEU-COMP-THERM-015-092 LEU-COMP-THERM-015-093 LEU-COMP-THERM-015-094 LEU-COMP-THERM-015-095 LEU-COMP-THERM-015-096 LEU-COMP-THERM-015-097 LEU-COMP-THERM-015-098 LEU-COMP-THERM-015-099 LEU-COMP-THERM-015-100 LEU-COMP-THERM-015-101 LEU-COMP-THERM-015-102 LEU-COMP-THERM-015-103 LEU-COMP-THERM-015-104_ LEU-COMP-THERM-015-105 LEU-COMP-THERM-015-106 LEU-COMP-THERM-015-107 LEU-COMP-THERM-015-108 LEU-COMP-THERM-015-109 LEU-COMP-THERM-015-110 LEU-COMP-THERM-015-111 LEU-COMP-THERM-015-112 LEU-COMP-THERM-015-113_ LEU-COMP-THERM-015-114 LEU-COMP-THERM-015-115 LEU-COMP-THERM-015-116 LEU-COMP-THERM-015-117

\begin{tabular}{|c|c|c|c|c|c|c|c|c|c|c|c|c|c|c|}
\hline 1 & 2 & 3 & 4 & 5 & 6 & 7 & 8 & 9 & 10 & 11 & 12 & 13 & 14 & 15 \\
\hline 0.99 & 0.96 & 0.98 & 0.93 & 0.91 & 0.88 & 0.90 & 0.92 & 0.82 & 0.82 & 0.90 & 0.81 & 0.53 & 0.73 & 0.63 \\
\hline 0.96 & 0.86 & 0.95 & 0.84 & 0.81 & 0.87 & 0.85 & 0.87 & 0.76 & 0.88 & 0.91 & 0.86 & 0.65 & 0.78 & 0.63 \\
\hline 0.92 & 0.82 & 0.90 & 0.82 & 0.79 & 0.88 & 0.84 & 0.84 & 0.75 & 0.88 & 0.89 & 0.84 & 0.61 & 0.70 & 0.53 \\
\hline 0.99 & 0.93 & 0.98 & 0.91 & 0.88 & 0.89 & 0.89 & 0.91 & 0.80 & 0.84 & 0.91 & 0.83 & 0.57 & 0.74 & 0.62 \\
\hline 0.99 & 0.93 & 0.98 & 0.91 & 0.88 & 0.89 & 0.89 & 0.91 & 0.81 & 0.84 & 0.91 & 0.83 & 0.57 & 0.74 & 0.62 \\
\hline 0.99 & 0.92 & 0.97 & 0.90 & 0.87 & 0.89 & 0.89 & 0.91 & 0.80 & 0.85 & 0.91 & 0.83 & 0.58 & 0.75 & 0.62 \\
\hline 0.99 & 0.93 & 0.98 & 0.91 & 0.88 & 0.89 & 0.89 & 0.91 & 0.81 & 0.84 & 0.91 & 0.83 & 0.57 & 0.74 & 0.62 \\
\hline 0.99 & 0.93 & 0.98 & 0.91 & 0.88 & 0.89 & 0.89 & 0.91 & 0.81 & 0.85 & 0.91 & 0.83 & 0.57 & 0.74 & 0.62 \\
\hline 0.99 & 0.93 & 0.97 & 0.91 & 0.88 & 0.89 & 0.89 & 0.91 & 0.80 & 0.84 & 0.91 & 0.83 & 0.57 & 0.74 & 0.62 \\
\hline 0.99 & 0.93 & 0.98 & 0.91 & 0.88 & 0.89 & 0.89 & 0.91 & 0.81 & 0.84 & 0.91 & 0.83 & 0.57 & 0.74 & 0.62 \\
\hline 0.99 & 0.93 & 0.98 & 0.91 & 0.88 & 0.89 & 0.89 & 0.91 & 0.81 & 0.84 & 0.91 & 0.83 & 0.57 & 0.74 & 0.62 \\
\hline 0.99 & 0.93 & 0.97 & 0.90 & 0.88 & 0.89 & 0.89 & 0.91 & 0.81 & 0.85 & 0.91 & 0.83 & 0.58 & 0.74 & 0.62 \\
\hline 0.97 & 0.89 & 0.96 & 0.87 & 0.84 & 0.88 & 0.87 & 0.89 & 0.78 & 0.86 & 0.91 & 0.84 & 0.61 & 0.75 & 0.61 \\
\hline 0.97 & 0.88 & 0.96 & 0.86 & 0.83 & 0.88 & 0.87 & 0.88 & 0.78 & 0.87 & 0.91 & 0.85 & 0.61 & 0.75 & 0.61 \\
\hline 0.97 & 0.89 & 0.96 & 0.87 & 0.84 & 0.88 & 0.87 & 0.89 & 0.78 & 0.87 & 0.91 & 0.85 & 0.61 & 0.75 & 0.61 \\
\hline 0.95 & 0.84 & 0.94 & 0.82 & 0.79 & 0.87 & 0.84 & 0.86 & 0.75 & 0.88 & 0.91 & 0.86 & 0.65 & 0.76 & 0.60 \\
\hline 0.97 & 0.88 & 0.96 & 0.86 & 0.83 & 0.89 & 0.87 & 0.89 & 0.78 & 0.88 & 0.92 & 0.86 & 0.63 & 0.76 & 0.61 \\
\hline 0.97 & 0.88 & 0.96 & 0.86 & 0.83 & 0.89 & 0.87 & 0.89 & 0.78 & 0.88 & 0.92 & 0.86 & 0.63 & 0.76 & 0.61 \\
\hline 0.97 & 0.87 & 0.96 & 0.85 & 0.82 & 0.89 & 0.86 & 0.88 & 0.77 & 0.88 & 0.92 & 0.86 & 0.64 & 0.76 & 0.61 \\
\hline 0.97 & 0.88 & 0.96 & 0.86 & 0.83 & 0.89 & 0.86 & 0.89 & 0.78 & 0.88 & 0.92 & 0.86 & 0.63 & 0.76 & 0.61 \\
\hline 0.95 & 0.84 & 0.94 & 0.83 & 0.79 & 0.88 & 0.84 & 0.86 & 0.75 & 0.89 & 0.91 & 0.87 & 0.66 & 0.77 & 0.61 \\
\hline 0.97 & 0.87 & 0.96 & 0.85 & 0.82 & 0.89 & 0.86 & 0.88 & 0.77 & 0.88 & 0.92 & 0.86 & 0.64 & 0.76 & 0.61 \\
\hline 0.96 & 0.86 & 0.95 & 0.84 & 0.81 & 0.88 & 0.86 & 0.88 & 0.77 & 0.88 & 0.92 & 0.86 & 0.64 & 0.76 & 0.61 \\
\hline 0.96 & 0.86 & 0.95 & 0.85 & 0.81 & 0.88 & 0.86 & 0.88 & 0.77 & 0.88 & 0.92 & 0.86 & 0.64 & 0.77 & 0.61 \\
\hline 0.99 & 0.93 & 0.97 & 0.91 & 0.88 & 0.89 & 0.89 & 0.91 & 0.81 & 0.85 & 0.91 & 0.83 & 0.58 & 0.75 & 0.62 \\
\hline 0.99 & 0.94 & 0.98 & 0.92 & 0.89 & 0.89 & 0.89 & 0.91 & 0.81 & 0.84 & 0.91 & 0.82 & 0.55 & 0.74 & 0.62 \\
\hline 0.99 & 0.93 & 0.98 & 0.91 & 0.88 & 0.89 & 0.89 & 0.91 & 0.81 & 0.84 & 0.91 & 0.83 & 0.57 & 0.74 & 0.62 \\
\hline 0.99 & 0.93 & 0.98 & 0.91 & 0.89 & 0.89 & 0.89 & 0.91 & 0.81 & 0.84 & 0.91 & 0.82 & 0.56 & 0.74 & 0.62 \\
\hline 0.98 & 0.92 & 0.97 & 0.90 & 0.87 & 0.89 & 0.88 & 0.91 & 0.80 & 0.85 & 0.91 & 0.83 & 0.58 & 0.75 & 0.62 \\
\hline 0.98 & 0.91 & 0.97 & 0.89 & 0.86 & 0.89 & 0.88 & 0.90 & 0.79 & 0.86 & 0.91 & 0.84 & 0.59 & 0.75 & 0.62 \\
\hline 0.93 & 0.82 & 0.92 & 0.80 & 0.76 & 0.84 & 0.80 & 0.83 & 0.71 & 0.85 & 0.88 & 0.83 & 0.68 & 0.79 & 0.63 \\
\hline 0.98 & 0.91 & 0.97 & 0.89 & 0.86 & 0.89 & 0.88 & 0.90 & 0.79 & 0.86 & 0.92 & 0.84 & 0.60 & 0.75 & 0.62 \\
\hline 0.98 & 0.90 & 0.97 & 0.88 & 0.85 & 0.89 & 0.88 & 0.90 & 0.79 & 0.86 & 0.92 & 0.85 & 0.60 & 0.75 & 0.61 \\
\hline 0.98 & 0.89 & 0.97 & 0.87 & 0.84 & 0.89 & 0.87 & 0.89 & 0.79 & 0.87 & 0.92 & 0.85 & 0.62 & 0.76 & 0.62 \\
\hline 0.97 & 0.89 & 0.96 & 0.87 & 0.83 & 0.89 & 0.87 & 0.89 & 0.78 & 0.88 & 0.92 & 0.86 & 0.62 & 0.76 & 0.61 \\
\hline 0.97 & 0.89 & 0.96 & 0.87 & 0.84 & 0.89 & 0.87 & 0.89 & 0.78 & 0.87 & 0.92 & 0.85 & 0.62 & 0.76 & 0.62 \\
\hline 0.93 & 0.81 & 0.92 & 0.79 & 0.76 & 0.87 & 0.82 & 0.85 & 0.73 & 0.90 & 0.91 & 0.87 & 0.68 & 0.77 & 0.59 \\
\hline 0.99 & 0.93 & 0.98 & 0.91 & 0.89 & 0.89 & 0.90 & 0.91 & 0.81 & 0.85 & 0.91 & 0.83 & 0.57 & 0.74 & 0.62 \\
\hline 0.99 & 0.93 & 0.98 & 0.92 & 0.89 & 0.89 & 0.90 & 0.92 & 0.82 & 0.85 & 0.91 & 0.83 & 0.56 & 0.74 & 0.62 \\
\hline 0.97 & 0.88 & 0.96 & 0.86 & 0.83 & 0.89 & 0.87 & 0.88 & 0.78 & 0.88 & 0.92 & 0.86 & 0.63 & 0.76 & 0.61 \\
\hline 0.94 & 0.81 & 0.93 & 0.80 & 0.77 & 0.88 & 0.83 & 0.85 & 0.74 & 0.90 & 0.92 & 0.88 & 0.68 & 0.77 & 0.59 \\
\hline 0.97 & 0.87 & 0.95 & 0.85 & 0.82 & 0.89 & 0.86 & 0.88 & 0.77 & 0.88 & 0.92 & 0.86 & 0.64 & 0.76 & 0.61 \\
\hline
\end{tabular}


Experiment

LEU-COMP-THERM-015-118 LEU-COMP-THERM-015-119 LEU-COMP-THERM-015-120 LEU-COMP-THERM-015-121 LEU-COMP-THERM-015-122 LEU-COMP-THERM-015-123 LEU-COMP-THERM-015-124_LEU-COMP-THERM-015-125 LEU-COMP-THERM-015-126 LEU-COMP-THERM-015-127 LEU-COMP-THERM-015-128 LEU-COMP-THERM-015-129 LEU-COMP-THERM-015-130 LEU-COMP-THERM-015-131_ LEU-COMP-THERM-015-132 LEU-COMP-THERM-015-133 LEU-COMP-THERM-015-134 LEU-COMP-THERM-015-135 LEU-COMP-THERM-015-136 LEU-COMP-THERM-015-137 LEU-COMP-THERM-015-138 LEU-COMP-THERM-015-139 LEU-COMP-THERM-015-140 LEU-COMP-THERM-015-141 LEU-COMP-THERM-015-142 LEU-COMP-THERM-015-143 LEU-COMP-THERM-015-144 LEU-COMP-THERM-015-145 LEU-COMP-THERM-015-146 LEU-COMP-THERM-015-147 LEU-COMP-THERM-015-148 LEU-COMP-THERM-015-149 LEU-COMP-THERM-015-150 LEU-COMP-THERM-015-151_ LEU-COMP-THERM-015-152 LEU-COMP-THERM-015-153 LEU-COMP-THERM-015-154_ LEU-COMP-THERM-015-155 LEU-COMP-THERM-015-156 LEU-COMP-THERM-015-157 LEU-COMP-THERM-015-158 LEU-COMP-THERM-015-159

\begin{tabular}{|c|c|c|c|c|c|c|c|c|c|c|c|c|c|c|}
\hline 1 & 2 & 3 & 4 & 5 & 6 & 7 & 8 & 9 & 10 & 11 & 12 & 13 & 14 & 15 \\
\hline 0.97 & 0.88 & 0.96 & 0.86 & 0.83 & 0.89 & 0.87 & 0.89 & 0.78 & 0.88 & 0.92 & 0.86 & 0.63 & 0.76 & 0.61 \\
\hline 0.97 & 0.88 & 0.96 & 0.86 & 0.83 & 0.89 & 0.87 & 0.89 & 0.78 & 0.88 & 0.92 & 0.86 & 0.63 & 0.76 & \\
\hline 0.97 & 0.88 & 0.96 & 0.86 & 0.83 & 0.89 & 0.87 & 0.89 & 0.78 & 0.88 & 0.92 & 0.86 & 0.63 & 0.76 & \\
\hline 0.97 & 0.87 & 0.96 & 0.85 & 0.82 & 0.89 & 0.86 & 0.88 & 0.77 & 0.88 & 0.92 & 0.86 & 0.63 & 0.76 & \\
\hline 0.97 & 0.87 & 0.96 & 0.85 & 0.82 & 0.89 & 0.86 & 0.88 & 0.78 & 0.89 & 0.92 & 0.87 & 0.64 & 0.76 & \\
\hline 0.97 & 0.87 & 0.96 & 0.85 & 0.82 & 0.89 & 0.86 & 0.88 & 0.77 & 0.88 & 0.92 & 0.86 & .63 & 0.76 & \\
\hline 0.97 & 0.87 & 0.96 & 0.86 & 0.83 & 0.89 & 0.86 & 0.88 & 0.78 & 0.89 & 0.92 & 0.86 & 0.63 & 0.76 & \\
\hline 0.96 & 0.85 & 0.95 & 0.84 & 0.81 & 0.89 & 0.86 & 0.87 & 0.77 & 0.89 & 0.92 & 0.87 & 0.66 & 0.77 & \\
\hline 0.99 & 0.96 & 0.98 & 0.94 & 0.92 & 0.90 & 0.91 & 0.92 & 0.84 & 0.83 & 0.91 & 0.81 & 0.52 & 0.71 & \\
\hline 0.96 & 0.88 & 0.95 & 0.85 & 0.81 & 0.86 & 0.84 & 0.87 & 0.75 & 0.85 & 0.89 & 0.83 & 0.62 & 0.77 & \\
\hline 0.99 & 0.94 & 0.98 & 0.92 & 0.89 & 0.90 & 0.90 & 0.92 & 0.82 & 0.85 & 0.92 & 0.83 & 0.56 & 0.73 & \\
\hline 0.94 & 0.81 & 0.92 & 0.80 & 0.77 & 0.88 & 0.84 & 0.85 & 0.75 & 0.91 & 0.92 & 0.88 & 0.67 & 0.76 & \\
\hline 0.99 & 0.95 & 0.98 & 0.93 & 0.91 & 0.90 & 0.91 & 0.92 & 0.83 & 0.84 & 0.91 & 0.83 & 0.54 & 0.72 & \\
\hline 0.96 & 0.86 & 0.95 & 0.84 & 0.81 & 0.89 & 0.86 & 0.88 & 0.78 & 0.90 & 0.93 & 0.87 & 0.64 & 0.75 & \\
\hline 0.94 & 0.83 & 0.93 & 0.81 & 0.78 & 0.88 & 0.84 & 0.86 & 0.75 & 0.90 & 92 & 0.88 & 0.67 & 0.76 & \\
\hline 0.99 & 0.94 & 0.98 & 0.92 & 0.89 & 0.89 & 0.90 & 0.92 & 0.82 & 0.84 & 91 & 0.83 & 0.55 & 0.73 & \\
\hline 0.99 & 0.94 & 0.98 & 0.91 & 0.89 & 0.89 & 0.89 & 0.91 & 0.81 & 0.84 & .91 & 0.83 & 0.56 & 0.74 & \\
\hline 0.95 & 0.84 & 0.94 & 0.83 & 0.80 & 0.89 & 0.85 & 0.87 & 0.77 & 0.90 & 0.92 & 0.88 & 0.65 & 0.76 & \\
\hline 0.99 & 0.95 & 0.98 & 0.93 & 0.91 & 0.90 & 0.91 & 0.92 & 0.83 & 0.84 & 0.91 & 0.82 & 0.54 & 0.72 & \\
\hline 0.99 & 94 & 0.98 & 0.92 & 0.89 & 0.90 & 0.90 & 0.92 & 0.82 & 0.85 & 2 & 0.83 & 0. & 0.73 & \\
\hline 0.99 & 0.93 & 0.98 & 0.91 & 0.88 & 0.90 & 0.90 & 0.91 & 0.82 & 0.86 & 0.92 & 0.84 & 0.57 & 0.74 & \\
\hline 0.98 & 0.92 & 0.97 & 0.90 & 0.87 & 0.90 & 0.89 & 0.91 & 0.81 & 0.86 & 0.92 & 0.85 & 0.58 & 0.74 & \\
\hline 0.92 & 0.80 & 0.91 & 0.77 & 0.74 & 0.83 & 0.79 & 0.82 & 0.70 & 0.86 & 0.88 & 0.84 & 0.69 & 0.78 & \\
\hline 0.92 & 0.80 & 0.91 & 0.77 & 0.73 & 0.83 & 0.79 & 0.82 & 0.70 & 0.86 & 0.88 & 0.84 & 0.69 & 0.78 & \\
\hline 0.97 & 0.87 & 0.96 & 0.86 & 0.83 & 0.90 & 0.87 & 0.89 & 0.78 & 0.89 & 0.93 & 0.87 & 0.62 & 0.75 & \\
\hline 0.99 & 0.94 & 0.98 & 0.93 & 0.91 & 0.91 & 0.91 & 0.92 & 0.84 & 0.85 & 0.92 & 0.83 & 0.55 & 0.73 & \\
\hline 0.99 & 0.94 & 0.98 & 0.93 & 0.91 & 0.91 & 0.91 & 0.93 & 0.84 & 0.85 & 0.92 & 0.84 & 0.55 & 0.73 & \\
\hline 0.98 & 0.93 & 0.97 & 0.92 & 0.89 & 0.90 & 0.90 & 0.91 & 0.83 & 0.86 & 0.92 & 0.84 & 0.55 & 0.72 & \\
\hline 0.94 & 0.83 & 0.93 & 0.82 & 0.79 & 0.89 & 0.85 & 0.86 & 0.77 & 0.91 & 0.92 & 0.88 & 0.64 & 0.74 & \\
\hline 0.99 & 0.94 & 0.98 & 0.93 & 0.91 & 0.91 & 0.91 & 0.92 & 0.84 & 0.86 & .93 & 0.84 & 0.55 & 0.72 & \\
\hline 0.99 & 0.94 & 0.98 & 0.92 & 0.90 & 0.91 & 0.91 & 0.92 & 0.84 & 0.86 & 0.93 & 0.84 & 0.55 & 0.72 & \\
\hline 0.96 & 0.86 & 0.94 & 0.85 & 0.82 & 0.90 & 0.87 & 0.88 & 0.79 & 0.90 & 0.93 & 0.88 & 0.63 & 0.74 & \\
\hline 0.96 & 0.87 & 0.95 & 0.86 & 0.83 & 0.90 & 0.87 & 0.88 & 0.79 & 0.90 & 0.93 & 0.88 & 0.62 & 0.74 & \\
\hline 0.92 & 0.79 & .90 & 0.78 & 0.76 & 0.88 & 0.82 & 0.83 & 0.74 & 0.91 & 0.91 & 0.88 & 0.66 & 0.73 & \\
\hline 0.95 & 0.84 & 0.94 & 0.82 & 0.79 & 0.88 & 0.85 & 0.86 & 0.76 & 0.90 & 0.92 & 0.88 & 0.65 & 0.76 & \\
\hline 0.99 & 0.93 & 0.98 & 0.91 & 0.89 & 0.91 & 0.91 & 0.92 & 0.83 & 0.87 & 0.93 & 0.85 & 0.57 & 0.73 & \\
\hline 0.98 & 0.90 & 0.96 & 0.89 & 0.85 & 0.90 & 0.88 & 0.90 & 0.79 & 0.87 & 0.92 & 0.85 & 0.59 & 0.74 & \\
\hline 0.98 & 0.91 & 0.97 & 0.90 & 0.87 & 0.90 & 0.89 & 0.91 & 0.81 & 0.86 & 0.92 & 0.84 & 0.58 & 0.73 & \\
\hline 0.96 & 0.87 & 0.95 & 0.86 & 0.82 & 0.89 & 0.86 & 0.88 & 0.77 & 0.88 & 0.92 & 0.85 & 0.62 & 0.74 & \\
\hline 0.96 & 0.86 & 0.94 & 0.85 & 0.82 & 0.89 & 0.86 & 0.88 & 0.77 & 0.88 & 0.92 & 0.86 & 0.62 & 0.74 & \\
\hline 0.91 & 0.78 & 0.89 & 0.77 & 0.73 & 0.86 & 0.80 & 0.82 & 0.71 & 0.89 & 0.89 & 0.86 & 0.67 & 0.74 & \\
\hline 0.99 & 0.94 & 0.98 & 0.93 & 0.90 & 0.90 & 0.91 & 0.92 & 0.83 & 0.85 & 0.92 & 0.83 & 0.56 & 0.74 & \\
\hline
\end{tabular}

A-14 
Experiment

LEU-COMP-THERM-015-160 LEU-COMP-THERM-015-161_ LEU-COMP-THERM-015-162 LEU-COMP-THERM-015-163 LEU-COMP-THERM-015-164 LEU-COMP-THERM-015-165 LEU-COMP-THERM-016-001_ LEU-COMP-THERM-016-002 LEU-COMP-THERM-016-003 LEU-COMP-THERM-016-004 LEU-COMP-THERM-016-005 LEU-COMP-THERM-016-006 LEU-COMP-THERM-016-007 LEU-COMP-THERM-016-008 EU-COMP-THERM-016-009 LEU-COMP-THERM-016-010 LEU-COMP-THERM-016-011 LEU-COMP-THERM-016-012 LEU-COMP-THERM-016-013 LEU-COMP-THERM-016-014 LEU-COMP-THERM-016-015 LEU-COMP-THERM-016-016 LEU-COMP-THERM-016-017 LEU-COMP-THERM-016-018 LEU-COMP-THERM-016-019 LEU-COMP-THERM-016-020 LEU-COMP-THERM-016-021 LEU-COMP-THERM-016-022 LEU-COMP-THERM-016-023 LEU-COMP-THERM-016-024 LEU-COMP-THERM-016-025 LEU-COMP-THERM-016-026 LEU-COMP-THERM-016-027 LEU-COMP-THERM-016-028 LEU-COMP-THERM-016-029 LEU-COMP-THERM-016-030 LEU-COMP-THERM-016-031 LEU-COMP-THERM-016-032 LEU-COMP-THERM-017-001 LEU-COMP-THERM-017-002 LEU-COMP-THERM-017-003 LEU-COMP-THERM-017-004_-

\begin{tabular}{|c|c|c|c|c|c|c|c|c|c|c|c|c|c|c|}
\hline 1 & 2 & 3 & 4 & 5 & 6 & 7 & 8 & 9 & 10 & 11 & 12 & 13 & 14 & 15 \\
\hline 0.99 & 0.95 & 0.98 & 0.93 & 0.91 & 0.90 & 0.91 & 0.93 & 0.84 & 0.84 & 0.92 & 0.83 & 0.54 & 0.73 & 0.61 \\
\hline 0.99 & 0.94 & 0.98 & 0.92 & 0.90 & 0.90 & 0.90 & 0.92 & 0.83 & 0.85 & 0.92 & 0.83 & 0.56 & 0.74 & 0.62 \\
\hline 0.99 & 0.94 & 0.98 & 0.92 & 0.89 & 0.90 & 0.90 & 0.92 & 0.82 & 0.85 & 0.92 & 0.84 & 0.56 & 0.74 & 0.62 \\
\hline 0.99 & 0.92 & 0.98 & 0.90 & 0.87 & 0.90 & 0.89 & 0.91 & 0.81 & 0.86 & 0.92 & 0.85 & 0.59 & 0.75 & 0.62 \\
\hline 0.99 & 0.93 & 0.98 & 0.91 & 0.89 & 0.90 & 0.90 & 0.92 & 0.82 & 0.85 & 0.92 & 0.84 & 0.57 & 0.74 & 0.62 \\
\hline 0.98 & 0.92 & 0.97 & 0.90 & 0.87 & 0.90 & 0.89 & 0.91 & 0.81 & 0.86 & 0.92 & 0.84 & 0.58 & 0.74 & 0.61 \\
\hline 0.94 & 0.87 & 0.92 & 0.87 & 0.85 & 0.90 & 0.88 & 0.88 & 0.80 & 0.87 & 0.91 & 0.84 & 0.57 & 0.69 & 0.54 \\
\hline 0.94 & 0.85 & 0.92 & 0.86 & 0.83 & 0.90 & 0.87 & 0.87 & 0.79 & 0.87 & 0.91 & 0.84 & 0.58 & 0.69 & 0.53 \\
\hline 0.94 & 0.86 & 0.92 & 0.86 & 0.83 & 0.90 & 0.87 & 0.87 & 0.79 & 0.87 & 0.90 & 0.84 & 0.57 & 0.69 & .53 \\
\hline 0.94 & 0.86 & 0.92 & 0.87 & 0.84 & 0.90 & 0.87 & 0.88 & 0.80 & 0.87 & 0.91 & 0.84 & 0.57 & 0.69 & .54 \\
\hline 0.94 & 0.86 & 0.92 & 0.87 & 0.84 & 0.90 & 0.87 & 0.87 & 0.79 & 0.87 & 0.91 & 0.84 & 0.57 & 0.69 & 0.5 \\
\hline 0.95 & 0.87 & 0.93 & 0.87 & 0.85 & 0.90 & 0.88 & 0.88 & 0.80 & 0.87 & 0.91 & 0.84 & 0.56 & 0.69 & \\
\hline 0.95 & 0.88 & 0.93 & 0.88 & 0.85 & 0.90 & 0.87 & 0.88 & 0.80 & 0.86 & 0.90 & 0.83 & 0.56 & 0.69 & .54 \\
\hline 0.94 & 0.85 & 0.92 & 0.85 & 0.83 & 0.89 & 0.86 & 0.86 & 0.78 & 0.87 & .90 & 0.84 & 0.58 & 0.69 & .53 \\
\hline 0.95 & 0.87 & 0.93 & 0.87 & 0.85 & 0.90 & 0.87 & 0.88 & 0.79 & 0.86 & .90 & 0.83 & 0.56 & 0.69 & .54 \\
\hline 0.95 & 0.87 & 0.93 & 0.87 & 0.84 & 0.90 & 0.87 & 0.87 & 0.79 & 0.87 & 0.90 & 0.84 & 0.57 & 0.69 & 0.54 \\
\hline 0.95 & 0.88 & 0.93 & 0.88 & 0.85 & 0.90 & 0.88 & 0.88 & 0.80 & 0.86 & 0.90 & 0.83 & 0.55 & 0.69 & 0.54 \\
\hline 0.95 & 0.87 & 0.93 & 0.87 & 0.85 & 0.90 & 0.87 & 0.88 & 0.79 & 0.86 & .90 & 0.83 & 0.56 & 0.69 & .54 \\
\hline 0.95 & 0.87 & 0.93 & 0.87 & 0.84 & 0.90 & 0.87 & 0.87 & 0.79 & 0.87 & .90 & 0.84 & 0.57 & 0.69 & .54 \\
\hline 0.95 & 0.87 & 0.93 & 0.87 & 0.85 & 0.90 & 0.87 & 0.88 & 0.79 & 0.86 & 0.90 & 0.83 & 0.56 & 0.69 & .54 \\
\hline 0.94 & 0.86 & 0.92 & 0.86 & 0.83 & 0.89 & 0.86 & 0.87 & 0.79 & 0.86 & 0.90 & 0.83 & 0.57 & 0.69 & 0.5 \\
\hline 0.94 & 0.87 & 0.92 & 0.87 & 0.84 & 0.89 & 0.87 & 0.87 & 0.79 & 0.86 & 0.90 & 0.83 & 0.56 & 0.69 & 0.54 \\
\hline 0.94 & 0.87 & 0.93 & 0.87 & 0.84 & 0.89 & 0.87 & 0.87 & 0.79 & 0.86 & 0.90 & 0.83 & 0.56 & 0.69 & 0.54 \\
\hline 0.94 & 0.87 & 0.93 & 0.87 & 0.85 & 0.90 & 0.87 & 0.87 & 0.79 & 0.86 & 0.90 & 0.83 & 0.56 & 0.69 & .54 \\
\hline 0.94 & 0.87 & 0.92 & 0.86 & 0.84 & 0.89 & 0.87 & 0.87 & 0.79 & 0.86 & 0.90 & 0.83 & 0.56 & 0.69 & .54 \\
\hline 0.94 & 0.87 & 0.92 & 0.87 & 0.84 & 0.89 & 0.87 & 0.87 & 0.79 & 0.86 & 0.90 & 0.83 & 0.56 & 0.69 & 0.54 \\
\hline 0.94 & 0.86 & 0.92 & 0.86 & 0.84 & 0.89 & 0.87 & 0.87 & 0.79 & 0.87 & 0.90 & 0.84 & 0.57 & 0.69 & 0.54 \\
\hline 0.95 & 0.87 & 0.93 & 0.87 & 0.85 & 0.90 & 0.87 & 0.88 & 0.79 & 0.86 & 0.90 & 0.83 & 0.56 & 0.69 & 0.54 \\
\hline 0.94 & 0.86 & 0.92 & 0.86 & 0.83 & 0.89 & 0.87 & 0.87 & 0.79 & 0.87 & 0.90 & 0.84 & 0.57 & 0.69 & .53 \\
\hline 0.95 & 0.88 & 0.93 & 0.87 & 0.85 & 0.90 & 0.87 & 0.88 & 0.79 & 0.86 & 0.90 & 0.83 & 0.56 & 0.69 & 0.54 \\
\hline 0.95 & 0.88 & 0.93 & 0.87 & 0.85 & 0.90 & 0.87 & 0.88 & 0.80 & 0.86 & 0.90 & 0.83 & 0.56 & 0.69 & 0.54 \\
\hline 0.95 & 0.88 & 0.93 & 0.88 & 0.86 & 0.90 & 0.88 & 0.88 & 0.80 & 0.86 & 0.90 & 0.83 & 0.55 & 0.69 & 0.54 \\
\hline 0.95 & 0.88 & 0.93 & 0.88 & 0.85 & 0.90 & 0.88 & 0.88 & 0.80 & 0.86 & 0.90 & 0.83 & 0.56 & 0.69 & 0.54 \\
\hline 0.94 & 0.86 & 0.92 & 0.86 & 0.84 & 0.89 & 0.87 & 0.87 & 0.79 & 0.86 & 0.90 & 0.83 & 0.56 & 0.69 & .53 \\
\hline 0.94 & 0.86 & 0.92 & 0.86 & 0.84 & 0.89 & 0.87 & 0.87 & 0.79 & 0.86 & 0.90 & 0.83 & 0.56 & 0.69 & 0.53 \\
\hline 0.94 & 0.87 & 0.92 & 0.87 & 0.84 & 0.89 & 0.87 & 0.87 & 0.79 & 0.86 & 0.90 & 0.83 & 0.56 & 0.69 & 0.54 \\
\hline 0.94 & 0.86 & 0.92 & 0.86 & 0.84 & 0.89 & 0.87 & 0.87 & 0.79 & 0.87 & 0.90 & 0.83 & 0.56 & 0.69 & 0.53 \\
\hline 0.94 & 0.87 & 0.92 & 0.87 & 0.84 & 0.89 & 0.87 & 0.87 & 0.79 & 0.86 & 0.90 & 0.83 & 0.56 & 0.69 & 0.5 \\
\hline 0.93 & 0.85 & 0.91 & 0.85 & 0.82 & 0.89 & 0.86 & 0.86 & 0.78 & 0.87 & 0.90 & 0.84 & 0.58 & 0.69 & \\
\hline 0.93 & 0.85 & 0.92 & 0.85 & 0.83 & 0.89 & 0.86 & 0.86 & 0.78 & 0.87 & 0.90 & 0.84 & 0.57 & 0.69 & 053 \\
\hline 0.93 & 0.85 & 0.91 & 0.85 & 0.82 & 0.89 & 0.86 & 0.86 & 0.78 & 0.87 & 0.90 & 0.84 & 0.57 & 0.69 & 0.53 \\
\hline 0.88 & 0.75 & 0.86 & 0.76 & 0.73 & 0.86 & 0.80 & 0.80 & 0.71 & 0.88 & 0.88 & 0.84 & 0.65 & 0.70 & \\
\hline
\end{tabular}


Experiment

LEU-COMP-THERM-017-005 LEU-COMP-THERM-017-006 LEU-COMP-THERM-017-007 LEU-COMP-THERM-017-008 LEU-COMP-THERM-017-009 LEU-COMP-THERM-017-010 LEU-COMP-THERM-017-011 LEU-COMP-THERM-017-012 LEU-COMP-THERM-017-013 LEU-COMP-THERM-017-014 LEU-COMP-THERM-017-015 LEU-COMP-THERM-017-016 LEU-COMP-THERM-017-017 LEU-COMP-THERM-017-018 LEU-COMP-THERM-017-019 LEU-COMP-THERM-017-020 LEU-COMP-THERM-017-021 LEU-COMP-THERM-017-022 LEU-COMP-THERM-017-023 LEU-COMP-THERM-017-024 LEU-COMP-THERM-017-025 LEU-COMP-THERM-017-026 LEU-COMP-THERM-017-027 EU-COMP-THERM-017-028 LEU-COMP-THERM-017-029 LEU-COMP-THERM-018-001_ LEU-COMP-THERM-020-001 LEU-COMP-THERM-020-002 LEU-COMP-THERM-020-003 LEU-COMP-THERM-020-004 LEU-COMP-THERM-020-005 LEU-COMP-THERM-020-006 LEU-COMP-THERM-020-007 LEU-COMP-THERM-021-001_ LEU-COMP-THERM-021-002 LEU-COMP-THERM-021-003 LEU-COMP-THERM-021-004 LEU-COMP-THERM-021-005 LEU-COMP-THERM-021-006 LEU-COMP-THERM-022-001 LEU-COMP-THERM-022-002 LEU-COMP-THERM-022-003

\begin{tabular}{|c|c|c|c|c|c|c|c|c|c|c|c|c|c|c|}
\hline 1 & 2 & 3 & 4 & 5 & 6 & 7 & 8 & 9 & 10 & 11 & 12 & 13 & 14 & 15 \\
\hline 0.90 & 0.79 & 0.88 & 0.80 & 0.77 & 0.87 & 0.82 & 0.83 & 0.74 & 0.88 & 0.89 & 0.84 & 0.63 & 0.70 & .5 \\
\hline 0.91 & 0.80 & 0.89 & 0.81 & 0.78 & 0.88 & 0.83 & 0.83 & 0.75 & 0.88 & 0.89 & 0.84 & 0.61 & 0.70 & \\
\hline 0.91 & 0.80 & 0.89 & 0.81 & 0.78 & 0.88 & 0.83 & 0.83 & 0.75 & 0.88 & 0.89 & 0.84 & 0.61 & 0.69 & \\
\hline 0.92 & 0.83 & 0.90 & 0.83 & 0.80 & 0.88 & 0.84 & 0.85 & 0.76 & 0.87 & 0.89 & 0.84 & 0.59 & 0.69 & \\
\hline 0.93 & 0.85 & 0.91 & 0.85 & 0.82 & 0.89 & 0.86 & 0.86 & 0.78 & 0.87 & 0.90 & 0.83 & 0.57 & 0.68 & \\
\hline 0.92 & 0.82 & 0.90 & 0.84 & 0.81 & 0.91 & 0.87 & 0.88 & 0.80 & 0.90 & 0.92 & 0.87 & 0.61 & 0.71 & \\
\hline 0.93 & 0.83 & 0.91 & 0.86 & 0.83 & 0.92 & 0.88 & 0.89 & 0.81 & 0.90 & 0.93 & 0.87 & 0.60 & 0.71 & \\
\hline 0.93 & 0.83 & 0.91 & 0.85 & 0.82 & 0.91 & 0.87 & 0.87 & 0.79 & 0.90 & 0.92 & 0.86 & 0.60 & 0.70 & \\
\hline 0.93 & 0.83 & 0.91 & 0.85 & 0.82 & 0.90 & 0.86 & 0.87 & 0.78 & 0.89 & 0.91 & 0.85 & 0.59 & 0.70 & \\
\hline 0.93 & 0.84 & 0.91 & 0.85 & 0.82 & 0.89 & 0.86 & 0.87 & 0.78 & 0.88 & 0.91 & 0.85 & 0.58 & 0.69 & \\
\hline 0.89 & 0.77 & 0.87 & 0.80 & 0.76 & 0.89 & 0.84 & 0.86 & 0.76 & 0.91 & 91 & 0.88 & 0.68 & 0.75 & \\
\hline 0.91 & 0.79 & 0.89 & 0.82 & 0.78 & 0.90 & 0.85 & 0.87 & 0.77 & 0.91 & 0.92 & 0.88 & 0.66 & 0.75 & \\
\hline 0.92 & 0.79 & 0.90 & 0.81 & 0.77 & 0.89 & 0.84 & 0.86 & 0.76 & 0.90 & 0.91 & 0.87 & 0.66 & 0.75 & \\
\hline 0.92 & 0.80 & 0.90 & 0.81 & 0.77 & 0.89 & 0.84 & 0.86 & 0.75 & 0.90 & 0.91 & 0.87 & 0.66 & 0.75 & \\
\hline 0.92 & 0.80 & 0.90 & 0.82 & 0.78 & 0.89 & 0.84 & 0.86 & 0.75 & 0.90 & 0.91 & 0.87 & 0.65 & 0.74 & \\
\hline 0.93 & 0.81 & 0.91 & 0.82 & 0.78 & 0.89 & 0.84 & 0.86 & 0.75 & 0.89 & 91 & 0.86 & 0.64 & 0.74 & \\
\hline 0.94 & 0.82 & 0.92 & 0.82 & 0.79 & 0.88 & 0.84 & 0.86 & 0.75 & 0.89 & 0.91 & 0.85 & 0.64 & 0.74 & \\
\hline 0.94 & 0.83 & 0.92 & 0.83 & 0.79 & 0.88 & 0.84 & 0.85 & 0.74 & 0.88 & 0.90 & 0.84 & 0.62 & 0.73 & \\
\hline 0.92 & 0.81 & 0.91 & 0.81 & 0.77 & 0.86 & 0.82 & 0.84 & 0.72 & 0.87 & 0.89 & 0.84 & 0.63 & 0.73 & \\
\hline 0.93 & 0.82 & 0.91 & 0.81 & 0.77 & 0.87 & 0.82 & 0.84 & 0.73 & 0.87 & 0.89 & 0.84 & 0. & 0.73 & \\
\hline 0.93 & 0.82 & 0.91 & 0.81 & 0.77 & 0.87 & 0.82 & 0.84 & 0.73 & 0.88 & 0.89 & 0.84 & 0.63 & 0.73 & \\
\hline 0.82 & 0.66 & 0.80 & 0.66 & 0.62 & 0.80 & 0.71 & 0.73 & 0.62 & 0.86 & 0.83 & 0.82 & 0.71 & 0.72 & \\
\hline 0.87 & 0.72 & 0.85 & 0.72 & 0.68 & 0.83 & 0.76 & 0.77 & 0.66 & 0.87 & 0.86 & 0.83 & 0.69 & 0.73 & \\
\hline 0.89 & 0.75 & 0.87 & 0.75 & 0.71 & 0.84 & 0.78 & 0.79 & 0.68 & 0.88 & 0.87 & 0.84 & 0.68 & 0.73 & \\
\hline 0.90 & 0.77 & 0.88 & 0.77 & 0.73 & 0.85 & 0.79 & 0.81 & 0.70 & 0.88 & 0.88 & 0.84 & 0.67 & 0.73 & \\
\hline 0.95 & 0.98 & 0.95 & 0.99 & 0.99 & 0.92 & 0.96 & 0.96 & 0.93 & 0.79 & 0.90 & 0.79 & 0.46 & 0.67 & \\
\hline 0.92 & 0.92 & 0.92 & 0.93 & 0.93 & 0.90 & 0.93 & 0.90 & 0.88 & 0.81 & 0.89 & 0.80 & 0.46 & 0.62 & \\
\hline 0.91 & 0.93 & 0.91 & 0.93 & 0.93 & 0.88 & 0.91 & 0.89 & 0.87 & 0.78 & 0.87 & 0.77 & 0.43 & 0.60 & \\
\hline 0.91 & 0.92 & 0.90 & 0.92 & 0.93 & 0.88 & 0.91 & 0.88 & 0.87 & 0.78 & 0.86 & 0.76 & 0.42 & 0.60 & \\
\hline 0.91 & 0.92 & 0.90 & 0.92 & 0.93 & 0.87 & 0.91 & 0.88 & 0.87 & 0.77 & 0.86 & 0.76 & 0.42 & 0.59 & \\
\hline 0.91 & 0.92 & 0.90 & 0.92 & 0.93 & 0.87 & 0.91 & 0.88 & 0.86 & 0.77 & 0.86 & 0.76 & 0.42 & 0.59 & \\
\hline 0.91 & 0.92 & 0.90 & 0.92 & 0.93 & 0.87 & 0.90 & 0.88 & 0.86 & 0.77 & 0.85 & 0.75 & 0.42 & 0.59 & \\
\hline 0.90 & 0.92 & 0.89 & 0.91 & 0.92 & 0.87 & 0.90 & 0.87 & 0.86 & 0.77 & 0.85 & 0.75 & 0.42 & 0.59 & \\
\hline 0.98 & 0.95 & 0.97 & 0.93 & 0.93 & 0.91 & 0.93 & 0.92 & 0.87 & 0.85 & 0.92 & 0.84 & 0.52 & 0.69 & \\
\hline 0.97 & 0.95 & 0.97 & 0.93 & 0.93 & 0.91 & 0.93 & 0.92 & 0.87 & 0.84 & 0.92 & 0.83 & 0.52 & 0.69 & \\
\hline 0.97 & 0.95 & 0.97 & 0.93 & 0.93 & 0.91 & 0.93 & 0.92 & 0.87 & 0.85 & 0.92 & 0.83 & 0.52 & 0.69 & \\
\hline 0.91 & 0.86 & 0.91 & 0.86 & 0.86 & 0.90 & 0.89 & 0.87 & 0.84 & 0.86 & 0.91 & 0.85 & 0.52 & 0.65 & \\
\hline 0.91 & 0.87 & 0.91 & 0.87 & 0.87 & 0.90 & 0.89 & 0.87 & 0.84 & 0.86 & 0.91 & 0.84 & 0.52 & 0.64 & \\
\hline 0.91 & 0.87 & 0.91 & 0.87 & 0.87 & 0.89 & 0.89 & 0.87 & 0.84 & 0.86 & 0.90 & 0.84 & 0.52 & 0.64 & \\
\hline 0.93 & 0.92 & 0.93 & 0.96 & 0.94 & 0.96 & 0.98 & 0.99 & 0.96 & 0.88 & 0.95 & 0.88 & 0.62 & 0.78 & \\
\hline 0.92 & 0.94 & 0.92 & 0.98 & 0.98 & 0.96 & 0.99 & 0.98 & 0.97 & 0.84 & 0.93 & 0.84 & 0.53 & 0.71 & \\
\hline 0.88 & 0.92 & 0.88 & 0.96 & 0.97 & 0.93 & 0.97 & 0.95 & 0.97 & 0.80 & 0.89 & 0.80 & 0.46 & 0.63 & \\
\hline
\end{tabular}


Experiment

LEU-COMP-THERM-022-004 LEU-COMP-THERM-022-005 LEU-COMP-THERM-022-006 LEU-COMP-THERM-022-007 LEU-COMP-THERM-023-001_ LEU-COMP-THERM-023-002 LEU-COMP-THERM-023-003 LEU-COMP-THERM-023-004 LEU-COMP-THERM-023-005 LEU-COMP-THERM-023-006 LEU-COMP-THERM-024-001 LEU-COMP-THERM-024-002 LEU-COMP-THERM-026-001 LEU-COMP-THERM-026-002 EU-COMP-THERM-026-003 LEU-COMP-THERM-026-004 LEU-COMP-THERM-026-005 LEU-COMP-THERM-026-006 LEU-COMP-THERM-027-001 LEU-COMP-THERM-027-002 EU-COMP-THERM-027-003 LEU-COMP-THERM-027-004 LEU-COMP-THERM-028-001 EU-COMP-THERM-028-002 LEU-COMP-THERM-028-003 LEU-COMP-THERM-028-004 LEU-COMP-THERM-028-005 LEU-COMP-THERM-028-006 LEU-COMP-THERM-028-007 LEU-COMP-THERM-028-008 LEU-COMP-THERM-028-009 LEU-COMP-THERM-028-010 LEU-COMP-THERM-028-011 LEU-COMP-THERM-028-012 LEU-COMP-THERM-028-013 LEU-COMP-THERM-028-014 LEU-COMP-THERM-028-015 LEU-COMP-THERM-028-016 LEU-COMP-THERM-028-017 LEU-COMP-THERM-028-018 LEU-COMP-THERM-028-019 LEU-COMP-THERM-028-020_

\begin{tabular}{|c|c|c|c|c|c|c|c|c|c|c|c|c|c|c|}
\hline 1 & 2 & 3 & 4 & 5 & 6 & 7 & 8 & 9 & 10 & 11 & 12 & 13 & 14 & 15 \\
\hline 0.86 & 0.89 & 0.86 & 0.94 & 0.95 & 0.92 & 0.95 & 0.93 & 0.95 & 0.79 & 0.87 & 0.78 & 0.44 & 0.60 & 51 \\
\hline 0.84 & 0.87 & 0.84 & 0.92 & 0.93 & 0.91 & 0.94 & 0.91 & 0.94 & 0.79 & 0.87 & 0.79 & 0.44 & 0.59 & \\
\hline 0.81 & 0.80 & 0.80 & 0.86 & 0.86 & 0.89 & 0.89 & 0.87 & 0.89 & 0.82 & 0.86 & 0.80 & 0.47 & 0.58 & \\
\hline 0.81 & 0.79 & 0.80 & 0.85 & 0.86 & 0.90 & 0.89 & 0.87 & 0.89 & 0.82 & 0.87 & 0.81 & 0.47 & 0.58 & \\
\hline 0.85 & 0.89 & 0.85 & 0.93 & 0.95 & 0.92 & 0.95 & 0.92 & 0.95 & 0.80 & 0.88 & 0.79 & 0.45 & 0.60 & \\
\hline 0.85 & 0.89 & 0.85 & 0.93 & 0.95 & 0.91 & 0.95 & 0.92 & 0.95 & 0.79 & 0.87 & 0.78 & 0.44 & 0.59 & \\
\hline 0.85 & 0.88 & 0.85 & 0.93 & 0.94 & 0.91 & 0.95 & 0.91 & 0.94 & 0.78 & 0.87 & 0.78 & 0.44 & 0.59 & \\
\hline 0.85 & 0.88 & 0.85 & 0.93 & 0.94 & 0.91 & 0.94 & 0.91 & 0.94 & 0.78 & 0.86 & 0.78 & 0.43 & 0.59 & \\
\hline 0.84 & 0.88 & 0.84 & 0.93 & 0.94 & 0.91 & 0.94 & 0.91 & 0.94 & 0.78 & 0.86 & 0.78 & 0.43 & 0.59 & \\
\hline 0.84 & 0.88 & 0.84 & 0.92 & 0.94 & 0.91 & 0.94 & 0.91 & 0.94 & 0.78 & 0.86 & 0.78 & .44 & 0.59 & \\
\hline 0.92 & 0.89 & 0.93 & 0.93 & 0.91 & 0.95 & 0.95 & 0.98 & 0.93 & 0.88 & 0.94 & 0.89 & 0.66 & 0.81 & \\
\hline 0.89 & 0.91 & 0.89 & 0.96 & 0.97 & 0.94 & 0.98 & 0.96 & 0.97 & 0.82 & 0.91 & 0.82 & 0.49 & 0.65 & \\
\hline 0.99 & 0.98 & 0.98 & 0.95 & 0.94 & 0.89 & 0.92 & 0.93 & 0.85 & 0.80 & 0.90 & 0.79 & 0.50 & 0.71 & \\
\hline 0.99 & 0.96 & 0.99 & 0.94 & 0.91 & 0.89 & 0.90 & 0.92 & 0.83 & 0.82 & 0.90 & 0.81 & 0.54 & 0.73 & \\
\hline 0.93 & 0.83 & 0.93 & 0.80 & 0.76 & 0.83 & 0.80 & 0.84 & 0.71 & 0.83 & 0.87 & 0.82 & 0.68 & 0.80 & \\
\hline 0.88 & 0.76 & 0.88 & 0.73 & 0.69 & 0.80 & 0.75 & 0.79 & 0.66 & 0.84 & 0.85 & 0.82 & 0.75 & 0.82 & \\
\hline 0.99 & 0.94 & 0.98 & 0.91 & 0.89 & 0.89 & 0.90 & 0.92 & 0.82 & 0.84 & 0.91 & 0.83 & 0.58 & 0.76 & \\
\hline 0.98 & 0.92 & 0.98 & 0.90 & 0.87 & 0.89 & 0.89 & 0.91 & 0.81 & 0.85 & 0.91 & 0.84 & 0.61 & 0.77 & \\
\hline 0.91 & 0.90 & 0.91 & 0.90 & 0.89 & 0.86 & 0.88 & 0.87 & 0.83 & 0.78 & 0.86 & 0.77 & 0.47 & 0.64 & \\
\hline 0.92 & 0.91 & 0.92 & 0.91 & 0.90 & 0.87 & 0.89 & 0.88 & 0.84 & 0.79 & 0.87 & 0.78 & 0.47 & 0.65 & \\
\hline 0.93 & 0.92 & 0.92 & 0.92 & 0.91 & 0.88 & 0.90 & 0.89 & 0.85 & 0.80 & 0.88 & 0.79 & 0.48 & 0.65 & \\
\hline 0.94 & 0.92 & 0.93 & 0.92 & 0.92 & 0.89 & 0.91 & 0.90 & 0.86 & 0.81 & 0.89 & 0.80 & 0.49 & 0.66 & \\
\hline 0.92 & 0.88 & 0.91 & 0.94 & 0.91 & 0.98 & 0.97 & 0.98 & 0.94 & 0.91 & 0.96 & 0.90 & 0.60 & 0.74 & \\
\hline 0.91 & 0.86 & 0.91 & 0.92 & 0.89 & 0.98 & 0.96 & 0.98 & 0.93 & 0.93 & 0.97 & 0.92 & 0.63 & 0.75 & \\
\hline 0.90 & 0.83 & 0.90 & 0.89 & 0.86 & 0.98 & 0.94 & 0.97 & 0.92 & 0.95 & 0.97 & 0.93 & 0.66 & 0.77 & \\
\hline 0.89 & 0.80 & 0.88 & 0.87 & 0.84 & 0.97 & 0.93 & 0.95 & 0.90 & 0.96 & 0.97 & 0.95 & 0.69 & 0.77 & \\
\hline 0.91 & 0.86 & 0.90 & 0.92 & 0.89 & 0.97 & 0.95 & 0.97 & 0.93 & 0.92 & 0.96 & 0.91 & 0.62 & 0.75 & \\
\hline 0.87 & 0.80 & 0.87 & 0.86 & 0.83 & 0.95 & 0.91 & 0.93 & 0.89 & 0.92 & 0.94 & 0.91 & 0.65 & 0.74 & \\
\hline 0.90 & 0.84 & 0.89 & 0.90 & 0.88 & 0.96 & 0.94 & 0.96 & 0.92 & 0.93 & 0.96 & 0.93 & 0.62 & 0.74 & \\
\hline 0.85 & 0.79 & 0.85 & 0.85 & 0.82 & 0.92 & 0.89 & 0.91 & 0.87 & 0.92 & 0.94 & 0.94 & 0.62 & 0.72 & \\
\hline 0.80 & 0.73 & 0.80 & 0.79 & 0.76 & 0.88 & 0.84 & 0.86 & 0.82 & 0.90 & 0.91 & 0.92 & 0.61 & 0.69 & \\
\hline 0.89 & 0.86 & 0.89 & 0.93 & 0.92 & 0.98 & 0.97 & 0.97 & 0.95 & 0.91 & 0.95 & 0.89 & 0.57 & 0.70 & \\
\hline 0.89 & 0.84 & 0.88 & 0.91 & 0.89 & 0.98 & 0.96 & 0.96 & 0.94 & 0.94 & 0.96 & 0.92 & 0.61 & 0.71 & \\
\hline 0.86 & 0.78 & 0.86 & 0.86 & 0.84 & 0.98 & 0.93 & 0.94 & 0.91 & 0.96 & 0.97 & 0.94 & 0.65 & 0.73 & \\
\hline 0.86 & 0.81 & 0.86 & 0.88 & 0.86 & 0.95 & 0.93 & 0.93 & 0.91 & 0.93 & 0.95 & 0.93 & 0.59 & 0.69 & \\
\hline 0.77 & 0.69 & 0.76 & 0.76 & 0.74 & 0.86 & 0.82 & 0.83 & 0.81 & 0.89 & 0.89 & 0.92 & 0.58 & 0.64 & \\
\hline 0.85 & 0.79 & 0.84 & 0.87 & 0.86 & 0.96 & 0.92 & 0.92 & 0.91 & 0.92 & 0.94 & 0.90 & 0.58 & 0.67 & \\
\hline 0.84 & 0.77 & 0.83 & 0.85 & 0.84 & 0.95 & 0.91 & 0.91 & 0.90 & 0.93 & 0.94 & 0.91 & 0.60 & 0.67 & \\
\hline 0.80 & 0.69 & 0.79 & 0.78 & 0.76 & 0.93 & 0.86 & 0.87 & 0.85 & 0.95 & 0.93 & 0.92 & 0.64 & 0.68 & \\
\hline 0.82 & 0.73 & 0.81 & 0.81 & 0.79 & 0.94 & 0.89 & 0.89 & 0.87 & 0.94 & 0.93 & 0.92 & 0.63 & 0.68 & \\
\hline 0.82 & 0.75 & 0.81 & 0.83 & 0.81 & 0.94 & 0.89 & 0.89 & 0.88 & 0.93 & 0.94 & 0.93 & 0.59 & 0.66 & \\
\hline 0.78 & 0.69 & 0.77 & 0.77 & 0.75 & 0.90 & 0.84 & 0.85 & 0.83 & 0.92 & 0.91 & 0.93 & 0.59 & 0.64 & \\
\hline
\end{tabular}
0.49

0.46 0.50 0.50 0.50 .49 49
71 0.56 62 0.68 68
65 66 .54
.54 0.55 0.55 .60 60 59 0.59
0.60
0.58 .58
.59 0.57
0.54
0.55 0.55 0.55 .54
54 .54 0.48
0.51 0.51 0.48 50
50 .47 
Experiment

LEU-COMP-THERM-029-001 LEU-COMP-THERM-029-002 LEU-COMP-THERM-029-003 LEU-COMP-THERM-029-004 LEU-COMP-THERM-029-005 LEU-COMP-THERM-029-006 LEU-COMP-THERM-029-007 LEU-COMP-THERM-029-008 LEU-COMP-THERM-029-009 LEU-COMP-THERM-029-010 LEU-COMP-THERM-029-011 LEU-COMP-THERM-029-012 LEU-COMP-THERM-030-001 LEU-COMP-THERM-030-002 LEU-COMP-THERM-030-003 LEU-COMP-THERM-030-004 LEU-COMP-THERM-030-005 LEU-COMP-THERM-030-006 LEU-COMP-THERM-030-007 LEU-COMP-THERM-030-008 LEU-COMP-THERM-030-009 LEU-COMP-THERM-030-010 LEU-COMP-THERM-030-011 LEU-COMP-THERM-030-012 LEU-COMP-THERM-031-001 LEU-COMP-THERM-031-002 LEU-COMP-THERM-031-003 LEU-COMP-THERM-031-004 LEU-COMP-THERM-031-005 LEU-COMP-THERM-031-006 LEU-COMP-THERM-032-001_ LEU-COMP-THERM-032-002 LEU-COMP-THERM-032-003 LEU-COMP-THERM-032-004 LEU-COMP-THERM-032-005 LEU-COMP-THERM-032-006 LEU-COMP-THERM-032-007 LEU-COMP-THERM-032-008 LEU-COMP-THERM-032-009 LEU-COMP-THERM-033-001_ LEU-COMP-THERM-033-002 LEU-COMP-THERM-033-003

\begin{tabular}{|c|c|c|c|c|c|c|c|c|c|c|c|c|c|c|}
\hline 1 & 2 & 3 & 4 & 5 & 6 & 7 & 8 & 9 & 10 & 11 & 12 & 13 & 14 & 15 \\
\hline 0.96 & 0.96 & 0.95 & 0.96 & 0.96 & 0.90 & 0.93 & 0.92 & 0.88 & 0.79 & 0.89 & 0.78 & 0.46 & 0.66 & 0.56 \\
\hline 0.96 & 0.96 & 0.95 & 0.96 & 0.96 & 0.90 & 0.93 & 0.92 & 0.88 & 0.79 & 0.89 & 0.78 & 0.46 & 0.66 & \\
\hline 0.96 & 0.96 & 0.95 & 0.96 & 0.96 & 0.90 & 0.93 & 0.92 & 0.88 & 0.79 & 0.89 & 0.78 & 0.46 & 0.66 & \\
\hline 0.96 & 0.96 & 0.95 & 0.96 & 0.96 & 0.90 & 0.93 & 0.92 & 0.88 & 0.79 & 0.89 & 0.79 & 0.46 & 0.66 & \\
\hline 0.96 & 0.96 & 0.95 & 0.96 & 0.96 & 0.90 & 0.93 & 0.92 & 0.88 & 0.80 & 0.89 & 0.79 & 0.46 & 0.66 & \\
\hline 0.96 & 0.96 & 0.95 & 0.95 & 0.95 & 0.90 & 0.93 & 0.92 & 0.88 & 0.80 & 0.89 & 0.79 & 0.47 & 0.67 & \\
\hline 0.96 & 0.96 & 0.95 & 0.95 & 0.95 & 0.90 & 0.93 & 0.92 & 0.88 & 0.80 & 0.89 & 0.79 & 0.47 & 0.66 & \\
\hline 0.95 & 0.96 & 0.95 & 0.95 & 0.95 & 0.90 & 0.93 & 0.92 & 0.88 & 0.80 & 0.89 & 0.79 & 0.47 & 0.66 & \\
\hline 0.95 & 0.96 & 0.95 & 0.96 & 0.96 & 0.89 & 0.93 & 0.92 & 0.88 & 0.78 & 0.88 & 0.78 & 0.45 & 0.65 & \\
\hline 0.95 & 0.96 & 0.94 & 0.96 & 0.96 & 0.89 & 0.93 & 0.92 & 0.88 & 0.78 & 0.88 & 0.77 & 0.45 & 0.65 & \\
\hline 0.95 & 0.96 & 0.94 & 0.96 & 0.96 & 0.89 & 0.93 & 0.92 & 0.88 & 0.78 & 0.88 & 0.77 & 0.45 & 0.65 & \\
\hline 0.95 & 0.96 & 0.94 & 0.96 & 0.96 & 0.89 & 0.93 & 0.91 & 0.88 & 0.78 & 0.88 & 0.77 & 0.45 & 0.65 & \\
\hline 0.98 & 0.92 & 0.97 & 0.90 & 0.87 & 0.89 & 0.88 & 0.91 & 0.80 & 0.84 & 0.91 & 0.83 & 0.57 & 0.74 & \\
\hline 0.99 & 0.93 & 0.97 & 0.91 & 0.88 & 0.88 & 0.89 & 0.91 & 0.80 & 0.84 & .90 & 0.82 & 0.56 & 0.74 & \\
\hline 0.99 & 0.93 & 0.98 & 0.91 & 0.88 & 0.88 & 0.89 & 0.91 & 0.80 & 0.83 & 0.90 & 0.82 & 0.55 & 0.73 & \\
\hline 0.99 & 0.94 & 0.98 & 0.91 & 0.89 & 0.88 & 0.89 & 0.91 & 0.81 & 0.83 & 0.90 & 0.81 & 0.55 & 0.73 & \\
\hline 0.99 & 0.94 & 0.98 & 0.92 & 0.89 & 0.89 & 0.89 & 0.91 & 0.81 & 0.83 & 0.90 & 0.82 & 0.55 & 0.73 & \\
\hline 0.99 & 0.94 & 0.98 & 0.92 & 0.89 & 0.89 & 0.89 & 0.91 & 0.81 & 0.83 & 0.90 & 0.82 & 0 . & 0.73 & 0. \\
\hline 0.99 & 0.94 & 0.98 & 0.92 & 0.89 & 0.89 & 0.89 & 0.91 & 0.81 & 0.83 & 0.90 & 0.81 & 0.55 & 0.73 & \\
\hline 0.99 & 0.94 & 0.98 & 0.92 & 0.89 & 0.89 & 0.89 & 0.91 & 0.81 & 0.83 & 0.91 & 0.82 & 0.55 & 0.73 & \\
\hline 0.99 & 0.94 & 0.98 & 0.92 & 0.89 & 0.89 & 0.89 & 0.91 & 0.81 & 0.83 & 0.90 & 0.81 & 0.54 & 0.73 & 0. \\
\hline 0.99 & 0.94 & 0.98 & 0.92 & 0.89 & 0.89 & 0.89 & 0.91 & 0.81 & 0.83 & 0.90 & 0.82 & 0.55 & 0.73 & \\
\hline 0.99 & 0.94 & 0.98 & 0.92 & 0.89 & 0.89 & 0.89 & 0.91 & 0.81 & 0.83 & 0.91 & 0.82 & 0.55 & 0.73 & 0. \\
\hline 0.99 & 0.94 & 0.98 & 0.92 & 0.89 & 0.89 & 0.89 & 0.91 & 0.81 & 0.84 & 0.91 & 0.82 & 0.55 & 0.73 & 0. \\
\hline 0.99 & 0.95 & 0.98 & 0.93 & 0.91 & 0.89 & 0.90 & 0.92 & 0.83 & 0.83 & 0.91 & 0.82 & 0.55 & 0.74 & \\
\hline 0.99 & 0.95 & 0.98 & 0.93 & 0.90 & 0.89 & 0.90 & 0.92 & 0.83 & 0.83 & 0.91 & 0.82 & 0.55 & 0.74 & \\
\hline 0.99 & 0.96 & 0.98 & 0.93 & 0.91 & 0.88 & 0.90 & 0.92 & 0.83 & 0.82 & 0.90 & 0.81 & 0.54 & 0.73 & \\
\hline 0.99 & 0.96 & 0.98 & 0.93 & 0.91 & 0.89 & 0.91 & 0.92 & 0.83 & 0.82 & 0.90 & 0.81 & 0.54 & 0.73 & 0.6 \\
\hline 0.99 & 0.96 & 0.98 & 0.93 & 0.91 & 0.89 & 0.91 & 0.92 & 0.83 & 0.82 & 0.91 & 0.81 & 0.54 & 0.73 & \\
\hline 0.99 & 0.96 & 0.98 & 0.93 & 0.91 & 0.89 & 0.90 & 0.92 & 0.83 & 0.82 & 0.91 & 0.81 & 0.54 & 0.73 & \\
\hline 0.93 & 0.92 & 0.93 & 0.96 & 0.95 & 0.96 & 0.97 & 0.99 & 0.95 & 0.87 & 0.94 & 0.87 & 0.61 & 0.77 & 0. \\
\hline 0.93 & 0.91 & 0.93 & 0.95 & 0.94 & 0.95 & 0.97 & 0.98 & 0.95 & 0.87 & 0.94 & 0.88 & 0.62 & 0.79 & \\
\hline 0.92 & 0.88 & 0.92 & 0.92 & 0.91 & 0.95 & 0.95 & 0.97 & 0.93 & 0.89 & 0.94 & 0.89 & 0.67 & 0.81 & \\
\hline 0.84 & 0.87 & 0.84 & 0.92 & 0.94 & 0.91 & 0.94 & 0.91 & 0.94 & 0.79 & 0.87 & 0.79 & 0.44 & 0.59 & \\
\hline 0.84 & 0.88 & 0.84 & 0.93 & 0.95 & 0.91 & 0.95 & 0.92 & 0.95 & 0.78 & 0.86 & 0.78 & 0.44 & 0.59 & 0. \\
\hline 0.85 & 0.89 & 0.85 & 0.94 & 0.95 & 0.91 & 0.95 & 0.92 & 0.95 & 0.78 & 0.86 & 0.78 & 0.44 & 0.59 & \\
\hline 0.80 & 0.79 & 0.80 & 0.85 & 0.86 & 0.90 & 0.89 & 0.87 & 0.89 & 0.83 & 0.87 & 0.81 & 0.48 & 0.58 & \\
\hline 0.81 & 0.82 & 0.81 & 0.87 & 0.89 & 0.90 & 0.91 & 0.88 & 0.91 & 0.81 & 0.87 & 0.80 & 0.46 & 0.58 & \\
\hline 0.82 & 0.84 & 0.82 & 0.89 & 0.90 & 0.91 & 0.92 & 0.89 & 0.92 & 0.81 & 0.87 & 0.80 & 0.46 & 0.58 & \\
\hline 0.74 & 0.57 & 0.72 & 0.58 & 0.53 & 0.72 & 0.64 & 0.65 & 0.53 & 0.80 & 0.76 & 0.76 & 0.73 & 0.75 & \\
\hline 0.74 & 0.58 & 0.7 & 0. & 0.54 & 0.73 & 0.65 & 0.66 & 0.54 & 0.80 & 0.76 & 0.76 & 0.74 & 0.77 & \\
\hline 0.75 & 0.58 & 0.73 & 0.59 & 0.54 & 0.73 & 0.64 & 0.66 & 0.54 & 0.80 & 0.76 & 0.76 & 0.73 & 0.77 & \\
\hline
\end{tabular}


Case

715

716

718
719

720

721

722

724
725

726

727

728

729

730

731
732

733
734

735

736

737

738

739

740

741

742

743

744

745

746

747

748

749

750

751

752

753

754

755

756
Experiment

LEU-COMP-THERM-033-004 LEU-COMP-THERM-033-005

LEU-COMP-THERM-033-006

LEU-COMP-THERM-033-007

LEU-COMP-THERM-033-008

LEU-COMP-THERM-033-009

LEU-COMP-THERM-033-010_

LEU-COMP-THERM-033-011

LEU-COMP-THERM-033-012

LEU-COMP-THERM-033-013

LEU-COMP-THERM-033-014

LEU-COMP-THERM-033-015

LEU-COMP-THERM-033-016

LEU-COMP-THERM-033-017

LEU-COMP-THERM-033-018

LEU-COMP-THERM-033-019

LEU-COMP-THERM-033-020

EU-COMP-THERM-033-021

LEU-COMP-THERM-033-022

LEU-COMP-THERM-033-023

LEU-COMP-THERM-033-024

LEU-COMP-THERM-033-025

LEU-COMP-THERM-033-026

LEU-COMP-THERM-033-027

LEU-COMP-THERM-033-028

LEU-COMP-THERM-033-029

LEU-COMP-THERM-033-030

LEU-COMP-THERM-033-031

LEU-COMP-THERM-033-032

LEU-COMP-THERM-033-033

LEU-COMP-THERM-033-034

LEU-COMP-THERM-033-035

LEU-COMP-THERM-033-036

LEU-COMP-THERM-033-037

LEU-COMP-THERM-033-038

LEU-COMP-THERM-033-039

LEU-COMP-THERM-033-040

LEU-COMP-THERM-033-041

LEU-COMP-THERM-033-042

LEU-COMP-THERM-033-043

LEU-COMP-THERM-033-044

LEU-COMP-THERM-033-045 $\begin{array}{ll}1 & 2\end{array}$

$\begin{array}{lll}0.74 & 0.58 & \mathbf{3}\end{array}$

$\begin{array}{lll}0.80 & 0.67 & 0.78\end{array}$

$\begin{array}{ll}0.78 & 0.67\end{array}$

$\begin{array}{llll}0.80 & 0.66 & 0.78 & 0.67\end{array}$

$\begin{array}{llll}0.81 & 0.69 & 0.80 & 0.70\end{array}$

$\begin{array}{llll}0.81 & 0.69 & 0.79 & 0.70\end{array}$

$\begin{array}{llll}0.80 & 0.69 & 0.79 & 0.70\end{array}$

$\begin{array}{llll}0.80 & 0.69 & 0.79 & 0.70\end{array}$

$\begin{array}{llll}0.81 & 0.69 & 0.79 & 0.70\end{array}$

$\begin{array}{llll}0.78 & 0.67 & 0.77 & 0.68\end{array}$

$\begin{array}{llll}0.66 & 0.52 & 0.65 & 0.53\end{array}$

$\begin{array}{llll}0.67 & 0.53 & 0.66 & 0.55\end{array}$

$\begin{array}{llll}0.67 & 0.54 & 0.66 & 0.55\end{array}$

\begin{tabular}{l|l|l|l}
0.84 & 0.72 & 0.83 & 0.72 \\
\hline
\end{tabular}

$\begin{array}{llll}0.84 & 0.73 & 0.83 & 0.72\end{array}$

\begin{tabular}{l|l|l|l|}
\hline 0.85 & 0.74 & 0.83 & 0.73 \\
\hline
\end{tabular}

$\begin{array}{llll}0.85 & 0.73 & 0.84 & 0.73\end{array}$

$\begin{array}{llll}0.84 & 0.72 & 0.83 & 0.72\end{array}$

$\begin{array}{llll}0.88 & 0.82 & 0.87 & 0.83 \\ 0.69 & 0.51 & 0.67 & 0.52\end{array}$

$\begin{array}{llll}0.88 & 0.51 & 0.67 & 0.52\end{array}$

$\begin{array}{llll}0.70 & 0.53 & 0.68 & 0.53\end{array}$

$\begin{array}{llll}0.69 & 0.51 & 0.67 & 0.52\end{array}$

$0.77 \quad 0.63$

$0.76 \quad 0.62$

$\begin{array}{ll}0.76 \quad 0.61 \\ 0.77 & 0.62\end{array}$

$0.77 \quad 0.62$

$0.79 \quad 0.66$

$0.78 \quad 0.64$

$\begin{array}{ll}0.81 & 0.68\end{array}$

$\begin{array}{ll}0.80 & 0.67 \\ 0.80 & 0.68\end{array}$

$0.78 \quad 0.65$

$0.78 \quad 0.66$

$0.79 \quad 0.66$

$0.79 \quad 0.66$

$0.80 \quad 0.68$

$\begin{array}{ll}0.80 & 0.69\end{array}$

$0.77 \quad 0.66$

$0.77 \quad 0.64$

$0.77 \quad 0.65$

$0.66-0.51$

$0.68 \quad 0.54$

$0.77 \quad 0.67$
0.75
0.75

$0.74 \quad 0.61$

$0.75 \quad 0.63$

$\begin{array}{lllll}0.76 & 0.65 & 0.61 & 0.77 & 0.70\end{array}$

$\begin{array}{lllll}0.79 & 0.69 & 0.66 & 0.78 & 0.73\end{array}$

$\begin{array}{lllll}0.78 & 0.68 & 0.64 & 0.77 & 0.72\end{array}$

$0.79 \quad 0.69$

0.77
0.77

$\begin{array}{ll}0.77 & 0.67 \\ 0.78 & 0.69\end{array}$

0.78
0.79

$0.76 \quad 0.67$

$0.75 \quad 0.65$

$\begin{array}{ll}0.76 & 0.66 \\ 0.65 & 0.53\end{array}$

$0.65 \quad 0.53$

$\begin{array}{lll}0.66 & 0.78 & 0.73 \\ 0.64 & 0.78 & 0.72\end{array}$

$\begin{array}{lll}0.62 & 0.76 & 0.70\end{array}$

$\begin{array}{lll}0.63 & 0.77 & 0.72\end{array}$

$\begin{array}{ccccccccccr}\mathbf{5} & \mathbf{6} & \mathbf{7} & \mathbf{8} & \mathbf{9} & \mathbf{1 0} & \mathbf{1 1} & \mathbf{1 2} & \mathbf{1 3} & \mathbf{1 4} & \mathbf{1 5} \\ 0.54 & 0.72 & 0.64 & 0.66 & 0.53 & 0.80 & 0.76 & 0.76 & 0.74 & 0.77 & 0.55\end{array}$

$\begin{array}{llllllllllll}0.64 & 0.78 & 0.72 & 0.73 & 0.62 & 0.82 & 0.81 & 0.78 & 0.68 & 0.76 & 0.56\end{array}$

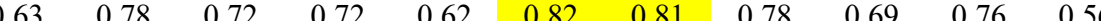

$\begin{array}{llllllllllll}0.63 & 0.78 & 0.72 & 0.72 & 0.61 & 0.82 & 0.81 & 0.78 & 0.69 & 0.76 & 0.55\end{array}$

$\begin{array}{llllllllllll}0.67 & 0.79 & 0.74 & 0.74 & 0.65 & 0.82 & 0.82 & 0.79 & 0.65 & 0.74 & 0.55\end{array}$

$\begin{array}{ll}0.75 & 0.74\end{array}$

$0.74 \quad 0.74$

$\begin{array}{llll}0.66 & 0.82 & 0.82 & 0.79\end{array}$

$\begin{array}{llll}0.65 & 0.82 & 0.82 & 0.79\end{array}$

$\begin{array}{llll}06 & 0.82 & 0.82 & 0.79\end{array}$

$\begin{array}{llll}0.65 & 0.81 & 0.81 & 0.78 \\ 0.53 & 0.76 & 0.72 & 0.73\end{array}$

$\begin{array}{llll}0.54 & 0.76 & 0.73 & 0.73\end{array}$

$\begin{array}{llll}0.55 & 0.77 & 0.73 & 0.73\end{array}$

\begin{tabular}{l|lll}
0.66 & 0.82 & 0.83 & 0.80
\end{tabular}

$\begin{array}{llll}0.65 & 0.82 & 0.83 & 0.79\end{array}$

\begin{tabular}{l|lll|}
0.65 & 0.82 & 0.83 & 0.79 \\
0.66 & 0.81 & 0.83 & 0.79
\end{tabular}

$\begin{array}{llll}0.66 & 0.82 & 0.83 & 0.80\end{array}$

$\begin{array}{llll}0.65 & 0.82 & 0.83 & 0.79\end{array}$

\begin{tabular}{|l|ll|l}
\hline 0.76 & 0.80 & 0.85 & 0.79 \\
\hline
\end{tabular}

$\begin{array}{llll}0.47 & 0.76 & 0.71 & 0.72\end{array}$

$\begin{array}{llll}0.49 & 0.76 & 0.72 & 0.73 \\ 0.47 & 0.76 & 0.71 & 0.72\end{array}$

\begin{tabular}{l|l|l|l}
0.58 & 0.81 & 0.79 & 0.77
\end{tabular}

$\begin{array}{llll}0.57 & 0.79 & 0.77 & 0.76\end{array}$

$\begin{array}{llll}0.55 & 0.79 & 0.77 & 0.76\end{array}$

\begin{tabular}{l|l|l|l|}
0.58 & 0.80 & 0.78 & 0.77
\end{tabular}

$\begin{array}{llll}0.62 & 0.83 & 0.81 & 0.79\end{array}$

\begin{tabular}{llll|l}
0.60 & 0.82 & 0.80 & 0.78
\end{tabular}

\begin{tabular}{llll|l}
0.64 & 0.82 & 0.81 & 0.78
\end{tabular}

$\begin{array}{llll}0.62 & 0.81 & 0.81 & 0.78\end{array}$

\begin{tabular}{llll|l}
0.63 & 0.81 & 0.81 & 0.78 \\
\hline
\end{tabular}

$\begin{array}{lllll}0.63 & 0.82 & 0.81 & 0.79\end{array}$

$\begin{array}{llll}0.63 & 0.82 & 0.81 & 0.79\end{array}$

\begin{tabular}{l|lll|l}
0.63 & 0.82 & 0.81 & 0.79
\end{tabular}

$\begin{array}{llll}0.62 & 0.81 & 0.81 & 0.78\end{array}$

$\begin{array}{llll}0.64 & 0.81 & 0.81 & 0.78\end{array}$

\begin{tabular}{|l|l|l|l|}
0.64 & 0.81 & 0.81 & 0.78 \\
\hline
\end{tabular}

$\begin{array}{lllll}0.64 & 0.81 & 0.80 & 0.78\end{array}$

$\begin{array}{llll}0.50 & 0.68 & 0.60 & 0.60 \\ 0.53 & 0.69 & 0.63 & 0.62\end{array}$

$\begin{array}{llll}0.53 & 0.69 & 0.63 & 0.62\end{array}$ 
Experiment

LEU-COMP-THERM-033-046 LEU-COMP-THERM-033-047 LEU-COMP-THERM-033-048 LEU-COMP-THERM-033-049 LEU-COMP-THERM-033-050 LEU-COMP-THERM-033-051 LEU-COMP-THERM-033-052 LEU-COMP-THERM-034-001 LEU-COMP-THERM-034-002 LEU-COMP-THERM-034-003 LEU-COMP-THERM-034-004 LEU-COMP-THERM-034-005 LEU-COMP-THERM-034-006 LEU-COMP-THERM-034-007 LEU-COMP-THERM-034-008 LEU-COMP-THERM-034-009 LEU-COMP-THERM-034-010 LEU-COMP-THERM-034-011 LEU-COMP-THERM-034-012 LEU-COMP-THERM-034-013 LEU-COMP-THERM-034-014 LEU-COMP-THERM-034-015 LEU-COMP-THERM-034-016 LEU-COMP-THERM-034-017 LEU-COMP-THERM-034-018 LEU-COMP-THERM-034-019 LEU-COMP-THERM-034-020 LEU-COMP-THERM-034-021 LEU-COMP-THERM-034-022 LEU-COMP-THERM-034-023 LEU-COMP-THERM-034-024 LEU-COMP-THERM-035-001 LEU-COMP-THERM-035-002 LEU-COMP-THERM-035-003 LEU-COMP-THERM-036-001 LEU-COMP-THERM-036-002 LEU-COMP-THERM-036-003 LEU-COMP-THERM-036-004 LEU-COMP-THERM-036-005 LEU-COMP-THERM-036-006 LEU-COMP-THERM-036-007 LEU-COMP-THERM-036-008 $\begin{array}{ll}0.66 & 0.52\end{array}$ $\begin{array}{llll}0.80 & 0.68 & 0.79 & 0.6 \\ 0.77 & 0.63 & 0.75 & 0.6\end{array}$

$\begin{array}{llll}0.79 & 0.67 & 0.78 & 0.66\end{array}$

\begin{tabular}{|l|l|l|l|}
\hline 0.87 & 0.80 & 0.86 & 0.80 \\
\hline
\end{tabular}

$\begin{array}{lllll}0.85 & 0.78 & 0.84 & 0.78\end{array}$

\begin{tabular}{|l|l|l|l|}
\hline 0.78 & 0.83 & 0.82 & 0.81 \\
\hline 0.76 & 0.81 & 0.79 & 0.79 \\
\hline
\end{tabular}

\begin{tabular}{|l|l|l|l|l|l|l|l|lll|}
0.77 & 0.83 & 0.81 & 0.81 & 0.73 & 0.82 & 0.85 & 0.80 & 0.61 & 0.77 & 0.6
\end{tabular}

$\begin{array}{lllllllllllllllll}0.98 & 0.96 & 0.98 & 0.95 & 0.94 & 0.92 & 0.94 & 0.94 & 0.88 & 0.84 & 0.92 & 0.83 & 0.51 & 0.69 & 0.58\end{array}$

$\begin{array}{llllllllllllllll}0.98 & 0.95 & 0.97 & 0.94 & 0.94 & 0.92 & 0.94 & 0.93 & 0.88 & 0.85 & 0.93 & 0.84 & 0.52 & 0.69 & 0.58\end{array}$

$\begin{array}{llllllllllllllll}0.98 & 0.95 & 0.97 & 0.94 & 0.93 & 0.92 & 0.94 & 0.93 & 0.88 & 0.85 & 0.93 & 0.84 & 0.52 & 0.70 & 0.58\end{array}$

$\begin{array}{lllllllllllllllll}0.98 & 0.95 & 0.98 & 0.94 & 0.93 & 0.92 & 0.93 & 0.93 & 0.87 & 0.85 & 0.92 & 0.84 & 0.52 & 0.69 & 0.58\end{array}$

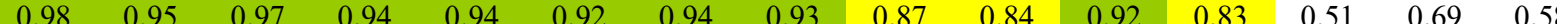

$\begin{array}{lllllllllllllllll}0.98 & 0.96 & 0.97 & 0.94 & 0.94 & 0.92 & 0.93 & 0.93 & 0.87 & 0.84 & 0.92 & 0.83 & 0.51 & 0.69 & 0.57\end{array}$

$\begin{array}{lllllllllllllllll}0.98 & 0.96 & 0.97 & 0.95 & 0.94 & 0.91 & 0.93 & 0.93 & 0.87 & 0.84 & 0.92 & 0.82 & 0.50 & 0.68 & 0.57\end{array}$

$\begin{array}{lllllllllllllll}0.98 & 0.96 & 0.97 & 0.95 & 0.94 & 0.91 & 0.93 & 0.93 & 0.87 & 0.83 & 0.91 & 0.82 & 0.50 & 0.68 & 0.57\end{array}$

$\begin{array}{llllllllllllllll}0.98 & 0.95 & 0.98 & 0.93 & 0.92 & 0.92 & 0.93 & 0.92 & 0.86 & 0.85 & 0.92 & 0.84 & 0.53 & 0.70 & 0.58\end{array}$

$\begin{array}{lllllllllllllll}0.98 & 0.94 & 0.98 & 0.93 & 0.92 & 0.92 & 0.92 & 0.92 & 0.86 & 0.86 & 0.92 & 0.84 & 0.53 & 0.70 & 0.58\end{array}$

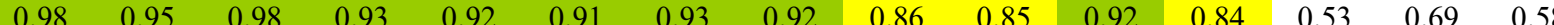

$\begin{array}{llllllllllllllll}0.98 & 0.95 & 0.98 & 0.93 & 0.92 & 0.91 & 0.92 & 0.92 & 0.86 & 0.85 & 0.92 & 0.84 & 0.53 & 0.70 & 0.58\end{array}$

$\begin{array}{lllllllllllllllll}0.98 & 0.95 & 0.98 & 0.93 & 0.92 & 0.91 & 0.93 & 0.92 & 0.86 & 0.85 & 0.92 & 0.84 & 0.52 & 0.69 & 0.58\end{array}$

$\begin{array}{lllllllllllllll}0.98 & 0.95 & 0.98 & 0.93 & 0.92 & 0.91 & 0.93 & 0.92 & 0.86 & 0.85 & 0.92 & 0.84 & 0.52 & 0.69 & 0.57\end{array}$

$\begin{array}{llllllllllllllll}0.98 & 0.96 & 0.98 & 0.95 & 0.94 & 0.92 & 0.94 & 0.94 & 0.88 & 0.85 & 0.93 & 0.84 & 0.52 & 0.70 & 0.58\end{array}$

$\begin{array}{llllllllllllllll}0.98 & 0.95 & 0.98 & 0.94 & 0.93 & 0.92 & 0.94 & 0.94 & 0.88 & 0.85 & 0.93 & 0.84 & 0.52 & 0.70 & 0.58\end{array}$

$\begin{array}{llllllllllllllll}0.98 & 0.95 & 0.97 & 0.94 & 0.93 & 0.92 & 0.94 & 0.93 & 0.87 & 0.85 & 0.93 & 0.84 & 0.52 & 0.70 & 0.58\end{array}$

$\begin{array}{lllllllllllllll}0.98 & 0.95 & 0.98 & 0.94 & 0.93 & 0.92 & 0.93 & 0.93 & 0.87 & 0.85 & 0.93 & 0.84 & 0.52 & 0.70 & 0.58\end{array}$

$\begin{array}{llllllllllllllll}0.98 & 0.95 & 0.98 & 0.93 & 0.93 & 0.92 & 0.93 & 0.93 & 0.87 & 0.85 & 0.93 & 0.84 & 0.53 & 0.70 & 0.58\end{array}$

$\begin{array}{lllllllllllllll}0.98 & 0.95 & 0.98 & 0.94 & 0.93 & 0.92 & 0.93 & 0.93 & 0.87 & 0.85 & 0.93 & 0.84 & 0.52 & 0.69 & 0.58\end{array}$

$\begin{array}{llllllllllllllll}0.98 & 0.95 & 0.97 & 0.94 & 0.93 & 0.92 & 0.93 & 0.93 & 0.87 & 0.85 & 0.92 & 0.84 & 0.52 & 0.69 & 0.58\end{array}$

$\begin{array}{llllllllllllllll}0.98 & 0.95 & 0.98 & 0.94 & 0.93 & 0.92 & 0.93 & 0.93 & 0.87 & 0.85 & 0.92 & 0.84 & 0.52 & 0.69 & 0.58\end{array}$

$\begin{array}{lllllllllllllll}0.98 & 0.95 & 0.98 & 0.94 & 0.93 & 0.92 & 0.93 & 0.93 & 0.87 & 0.84 & 0.92 & 0.83 & 0.51 & 0.69 & 0.58\end{array}$

$\begin{array}{llllllllllllllll}0.98 & 0.95 & 0.98 & 0.94 & 0.93 & 0.92 & 0.93 & 0.93 & 0.87 & 0.84 & 0.92 & 0.83 & 0.51 & 0.69 & 0.57\end{array}$

$\begin{array}{llllllllllllllll}0.98 & 0.91 & 0.96 & 0.89 & 0.85 & 0.88 & 0.87 & 0.89 & 0.78 & 0.84 & 0.90 & 0.82 & 0.57 & 0.73 & 0.59\end{array}$

$\begin{array}{lllllllllllllll}0.98 & 0.90 & 0.96 & 0.88 & 0.85 & 0.88 & 0.87 & 0.89 & 0.78 & 0.85 & 0.90 & 0.82 & 0.57 & 0.73 & 0.59\end{array}$

$\begin{array}{lllllllllllllllll}0.95 & 0.88 & 0.94 & 0.86 & 0.83 & 0.86 & 0.85 & 0.87 & 0.76 & 0.85 & 0.90 & 0.85 & 0.57 & 0.72 & 0.58\end{array}$

$\begin{array}{llllllllllllllllll}0.98 & 0.91 & 0.97 & 0.92 & 0.88 & 0.93 & 0.92 & 0.94 & 0.84 & 0.89 & 0.94 & 0.87 & 0.62 & 0.77 & 0.63\end{array}$

$\begin{array}{llllllllllllllll}0.97 & 0.89 & 0.96 & 0.90 & 0.87 & 0.92 & 0.91 & 0.93 & 0.83 & 0.89 & 0.94 & 0.87 & 0.63 & 0.77 & 0.63\end{array}$

$\begin{array}{lllllllllllllllll}0.96 & 0.88 & 0.95 & 0.89 & 0.86 & 0.91 & 0.90 & 0.93 & 0.83 & 0.89 & 0.94 & 0.87 & 0.63 & 0.77 & 0.62\end{array}$

$\begin{array}{llllllllllllllll}0.99 & 0.94 & 0.98 & 0.91 & 0.89 & 0.89 & 0.89 & 0.91 & 0.81 & 0.84 & 0.91 & 0.82 & 0.56 & 0.74 & 0.61\end{array}$

$\begin{array}{lllllllllllllllll}0.98 & 0.92 & 0.97 & 0.91 & 0.88 & 0.90 & 0.90 & 0.92 & 0.82 & 0.86 & 0.92 & 0.84 & 0.58 & 0.75 & 0.62\end{array}$

$\begin{array}{llllllllllllllll}0.98 & 0.91 & 0.97 & 0.91 & 0.88 & 0.90 & 0.90 & 0.92 & 0.82 & 0.86 & 0.92 & 0.85 & 0.59 & 0.76 & 0.62\end{array}$

$\begin{array}{llllllllllllllll}0.97 & 0.90 & 0.96 & 0.90 & 0.87 & 0.90 & 0.90 & 0.92 & 0.82 & 0.87 & 0.93 & 0.86 & 0.61 & 0.77 & 0.63\end{array}$ 
Experiment

LEU-COMP-THERM-036-009 LEU-COMP-THERM-036-010 LEU-COMP-THERM-036-011 LEU-COMP-THERM-036-012 LEU-COMP-THERM-036-013 LEU-COMP-THERM-036-014 LEU-COMP-THERM-036-015 LEU-COMP-THERM-036-016 LEU-COMP-THERM-036-017 LEU-COMP-THERM-036-018 LEU-COMP-THERM-036-019 LEU-COMP-THERM-036-020 LEU-COMP-THERM-036-021 LEU-COMP-THERM-036-022 LEU-COMP-THERM-036-023 LEU-COMP-THERM-036-024 LEU-COMP-THERM-036-025 LEU-COMP-THERM-036-026 LEU-COMP-THERM-036-027 LEU-COMP-THERM-036-028 LEU-COMP-THERM-036-029 LEU-COMP-THERM-036-030 LEU-COMP-THERM-036-031 LEU-COMP-THERM-036-032 LEU-COMP-THERM-036-033 LEU-COMP-THERM-036-034 LEU-COMP-THERM-036-035 LEU-COMP-THERM-036-036 LEU-COMP-THERM-036-037 LEU-COMP-THERM-036-038 LEU-COMP-THERM-036-039 LEU-COMP-THERM-036-040 LEU-COMP-THERM-036-041 LEU-COMP-THERM-036-042 LEU-COMP-THERM-036-043 LEU-COMP-THERM-036-044 LEU-COMP-THERM-036-045 LEU-COMP-THERM-036-046 LEU-COMP-THERM-036-047 LEU-COMP-THERM-036-048 LEU-COMP-THERM-036-049 LEU-COMP-THERM-036-050_-

\begin{tabular}{|c|c|c|c|c|c|c|c|c|c|c|c|c|c|c|}
\hline 1 & 2 & 3 & 4 & 5 & 6 & 7 & 8 & 9 & 10 & 11 & 12 & 13 & 14 & 15 \\
\hline 0.97 & 0.91 & 0.96 & 0.91 & 0.87 & 0.91 & 0.90 & 0.93 & 0.82 & 0.87 & 0.93 & 0.86 & 0.60 & 0.76 & 0.63 \\
\hline 0.98 & 0.92 & 0.97 & 0.91 & 0.88 & 0.91 & 0.91 & 0.93 & 0.83 & 0.87 & 0.93 & 0.85 & 0.60 & 0.76 & 0.63 \\
\hline 0.98 & 0.92 & 0.97 & 0.91 & 0.88 & 0.91 & 0.91 & 0.93 & 0.83 & 0.87 & 0.93 & 0.85 & 0.59 & 0.76 & 0.63 \\
\hline 0.97 & 0.88 & 0.96 & 0.88 & 0.85 & 0.91 & 0.89 & 0.91 & 0.81 & 0.89 & 0.93 & 0.87 & 0.64 & 0.78 & 0.62 \\
\hline 0.98 & 0.89 & 0.97 & 0.88 & 0.85 & 0.89 & 0.88 & 0.90 & 0.80 & 0.88 & 0.92 & 0.86 & 0.62 & 0.76 & 0.62 \\
\hline 0.96 & 0.87 & 0.95 & 0.86 & 0.83 & 0.90 & 0.88 & 0.90 & 0.79 & 0.89 & 0.93 & 0.87 & 0.64 & 0.77 & 0.62 \\
\hline 0.95 & 0.86 & 0.94 & 0.86 & 0.83 & 0.90 & 0.88 & 0.90 & 0.80 & 0.90 & 0.93 & 0.88 & 0.66 & 0.78 & 0.62 \\
\hline 0.91 & 0.78 & 0.89 & 0.77 & 0.73 & 0.85 & 0.79 & 0.82 & 0.69 & 0.87 & 0.88 & 0.83 & 0.66 & 0.74 & 0.55 \\
\hline 0.91 & 0.78 & 0.89 & 0.77 & 0.72 & 0.84 & 0.79 & 0.81 & 0.69 & 0.87 & 0.87 & 0.83 & 0.65 & 0.74 & 0.55 \\
\hline 0.92 & 0.80 & 0.90 & 0.79 & 0.75 & 0.85 & 0.80 & 0.83 & 0.70 & 0.87 & 0.88 & 0.83 & 0.64 & 0.74 & 0.56 \\
\hline 0.92 & 0.81 & 0.90 & 0.80 & 0.75 & 0.85 & 0.81 & 0.83 & 0.71 & 0.87 & 0.88 & 0.83 & 0.64 & 0.74 & 0.56 \\
\hline 0.93 & 0.81 & 0.90 & 0.80 & 0.75 & 0.85 & 0.80 & 0.83 & 0.70 & 0.86 & 0.88 & 0.83 & 0.64 & 0.73 & 0.56 \\
\hline 0.93 & 0.81 & 0.90 & 0.80 & 0.75 & 0.85 & 0.81 & 0.83 & 0.71 & 0.87 & 0.88 & 0.83 & 0.64 & 0.73 & 0.56 \\
\hline 0.93 & 0.81 & 0.90 & 0.80 & 0.76 & 0.85 & 0.81 & 0.83 & 0.71 & 0.87 & 0.88 & 0.83 & 0.64 & 0.73 & 0.56 \\
\hline 0.92 & 0.80 & 0.90 & 0.79 & 0.74 & 0.85 & 0.80 & 0.82 & 0.70 & 0.87 & 0.88 & 0.83 & 0.64 & 0.74 & 0.56 \\
\hline 0.95 & 0.84 & 0.93 & 0.83 & 0.79 & 0.86 & 0.83 & 0.85 & 0.73 & 0.86 & 0.89 & 0.83 & 0.62 & 0.74 & 0.57 \\
\hline 0.88 & 0.77 & 0.86 & 0.78 & 0.75 & 0.85 & 0.80 & 0.80 & 0.72 & 0.87 & 0.88 & 0.83 & 0.58 & 0.66 & 0.49 \\
\hline 0.88 & 0.77 & 0.86 & 0.78 & 0.75 & 0.85 & 0.80 & 0.80 & 0.72 & 0.87 & 0.87 & 0.83 & 0.58 & 0.66 & 0.49 \\
\hline 0.99 & 0.93 & 0.98 & 0.91 & 0.88 & 0.89 & 0.89 & 0.91 & 0.81 & 0.84 & 0.91 & 0.83 & 0.57 & 0.74 & 0.62 \\
\hline 0.99 & 0.93 & 0.97 & 0.90 & 0.88 & 0.89 & 0.89 & 0.91 & 0.80 & 0.85 & 0.91 & 0.83 & 0.57 & 0.74 & 0.62 \\
\hline 0.98 & 0.91 & 0.97 & 0.89 & 0.86 & 0.89 & 0.88 & 0.90 & 0.80 & 0.86 & 0.92 & 0.84 & 0.59 & 0.75 & 0.62 \\
\hline 0.98 & 0.91 & 0.97 & 0.89 & 0.86 & 0.89 & 0.88 & 0.90 & 0.80 & 0.86 & 0.92 & 0.85 & 0.59 & 0.75 & 0.62 \\
\hline 0.99 & 0.93 & 0.98 & 0.91 & 0.88 & 0.89 & 0.89 & 0.91 & 0.81 & 0.85 & 0.91 & 0.83 & 0.57 & 0.74 & 0.62 \\
\hline 0.99 & 0.93 & 0.98 & 0.91 & 0.88 & 0.89 & 0.89 & 0.91 & 0.81 & 0.84 & 0.91 & 0.83 & 0.56 & 0.74 & 0.62 \\
\hline 0.99 & 0.93 & 0.98 & 0.91 & 0.88 & 0.89 & 0.89 & 0.91 & 0.81 & 0.84 & 0.91 & 0.83 & 0.57 & 0.74 & 0.62 \\
\hline 0.99 & 0.93 & 0.98 & 0.91 & 0.88 & 0.89 & 0.89 & 0.91 & 0.81 & 0.84 & 0.91 & 0.83 & 0.56 & 0.74 & 0.62 \\
\hline 0.98 & 0.92 & 0.97 & 0.89 & 0.87 & 0.89 & 0.88 & 0.90 & 0.80 & 0.86 & 0.92 & 0.85 & 0.59 & 0.75 & 0.62 \\
\hline 0.98 & 0.91 & 0.97 & 0.89 & 0.86 & 0.89 & 0.88 & 0.90 & 0.80 & 0.86 & 0.92 & 0.84 & 0.58 & 0.75 & 0.62 \\
\hline 0.99 & 0.93 & 0.97 & 0.91 & 0.88 & 0.89 & 0.89 & 0.91 & 0.80 & 0.85 & 0.91 & 0.83 & 0.57 & 0.74 & 0.62 \\
\hline 0.98 & 0.91 & 0.97 & 0.89 & 0.86 & 0.89 & 0.88 & 0.90 & 0.80 & 0.86 & 0.92 & 0.85 & 0.59 & 0.75 & 0.62 \\
\hline 0.98 & 0.91 & 0.97 & 0.89 & 0.86 & 0.89 & 0.88 & 0.90 & 0.80 & 0.86 & 0.92 & 0.85 & 0.59 & 0.75 & 0.62 \\
\hline 0.99 & 0.93 & 0.98 & 0.91 & 0.88 & 0.89 & 0.89 & 0.91 & 0.80 & 0.84 & 0.91 & 0.83 & 0.57 & 0.74 & 0.62 \\
\hline 0.98 & 0.91 & 0.97 & 0.89 & 0.86 & 0.89 & 0.88 & 0.90 & 0.79 & 0.86 & 0.92 & 0.85 & 0.59 & 0.75 & 0.62 \\
\hline 0.98 & 0.91 & 0.97 & 0.89 & 0.86 & 0.89 & 0.88 & 0.90 & 0.80 & 0.86 & 0.92 & 0.85 & 0.59 & 0.75 & 0.62 \\
\hline 0.99 & 0.92 & 0.97 & 0.90 & 0.88 & 0.89 & 0.89 & 0.91 & 0.81 & 0.85 & 0.91 & 0.83 & 0.58 & 0.75 & 0.62 \\
\hline 0.99 & 0.93 & 0.97 & 0.90 & 0.88 & 0.89 & 0.89 & 0.91 & 0.81 & 0.85 & 0.92 & 0.84 & 0.57 & 0.74 & 0.62 \\
\hline 0.99 & 0.93 & 0.98 & 0.91 & 0.88 & 0.89 & 0.89 & 0.91 & 0.81 & 0.85 & 0.91 & 0.83 & 0.57 & 0.74 & 0.62 \\
\hline 0.93 & 0.81 & 0.91 & 0.80 & 0.76 & 0.86 & 0.81 & 0.83 & 0.71 & 0.87 & 0.89 & 0.83 & 0.64 & 0.73 & 0.56 \\
\hline 0.92 & 0.80 & 0.90 & 0.79 & 0.74 & 0.85 & 0.80 & 0.83 & 0.70 & 0.87 & 0.88 & 0.83 & 0.64 & 0.74 & 0.56 \\
\hline 0.95 & 0.86 & 0.93 & 0.84 & 0.80 & 0.87 & 0.84 & 0.86 & 0.74 & 0.86 & 0.89 & 0.83 & 0.61 & 0.74 & 0.58 \\
\hline 0.94 & 0.84 & 0.92 & 0.82 & 0.78 & 0.86 & 0.83 & 0.85 & 0.73 & 0.86 & 0.89 & 0.83 & 0.62 & 0.74 & 0.57 \\
\hline 0.98 & 0.89 & 0.96 & 0.88 & 0.85 & 0.90 & 0.88 & 0.90 & 0.80 & 0.88 & 0.93 & 0.86 & 0.62 & 0.76 & 0.61 \\
\hline
\end{tabular}


Experiment

LEU-COMP-THERM-036-051 LEU-COMP-THERM-036-052 LEU-COMP-THERM-036-053 LEU-COMP-THERM-036-054 LEU-COMP-THERM-036-055 LEU-COMP-THERM-036-056 LEU-COMP-THERM-036-057 LEU-COMP-THERM-036-058 LEU-COMP-THERM-036-059 LEU-COMP-THERM-036-060 LEU-COMP-THERM-036-061 LEU-COMP-THERM-036-062 LEU-COMP-THERM-036-063 LEU-COMP-THERM-036-064 LEU-COMP-THERM-036-065 LEU-COMP-THERM-036-066 LEU-COMP-THERM-036-067 LEU-COMP-THERM-036-068 LEU-COMP-THERM-036-069 LEU-COMP-THERM-037-001_ LEU-COMP-THERM-037-002 LEU-COMP-THERM-037-003 LEU-COMP-THERM-037-004 LEU-COMP-THERM-037-005 LEU-COMP-THERM-037-006 LEU-COMP-THERM-037-007 LEU-COMP-THERM-037-008 LEU-COMP-THERM-037-009 LEU-COMP-THERM-037-010 LEU-COMP-THERM-037-011 LEU-COMP-THERM-038-001_ LEU-COMP-THERM-038-002 LEU-COMP-THERM-038-003 LEU-COMP-THERM-038-004 LEU-COMP-THERM-038-005 LEU-COMP-THERM-038-006 LEU-COMP-THERM-038-007 LEU-COMP-THERM-038-008 LEU-COMP-THERM-038-009 LEU-COMP-THERM-038-010 LEU-COMP-THERM-038-011 LEU-COMP-THERM-038-012_-

\begin{tabular}{|c|c|c|c|c|c|c|c|c|c|c|c|c|c|c|}
\hline 1 & 2 & 3 & 4 & 5 & 6 & 7 & 8 & 9 & 10 & 11 & 12 & 13 & 14 & 15 \\
\hline 0.98 & 0.91 & 0.97 & 0.90 & 0.87 & 0.90 & 0.89 & 0.91 & 0.81 & 0.87 & 0.92 & 0.85 & 0.60 & 0.75 & \\
\hline 0.97 & 0.89 & 0.96 & 0.88 & 0.85 & 0.90 & 0.88 & 0.90 & 0.80 & 0.88 & 0.93 & 0.86 & 0.62 & 0.75 & \\
\hline 0.98 & 0.92 & 0.97 & 0.91 & 0.88 & 0.91 & 0.90 & 0.92 & 0.82 & 0.87 & 0.93 & 0.85 & 0.59 & 0.74 & \\
\hline 0.99 & 0.92 & 0.97 & 0.91 & 0.89 & 0.91 & 0.90 & 0.92 & 0.82 & 0.87 & 0.93 & 0.85 & 0.58 & 0.75 & \\
\hline 0.99 & 0.93 & 0.98 & 0.92 & 0.89 & 0.91 & 0.91 & 0.92 & 0.83 & 0.86 & 0.93 & 0.84 & 0.58 & 0.74 & \\
\hline 0.97 & 0.89 & 0.96 & 0.88 & 0.85 & 0.90 & 0.88 & 0.89 & 0.80 & 0.88 & 0.93 & 0.86 & 0.62 & 0.75 & \\
\hline 0.98 & 0.89 & 0.96 & 0.88 & 0.85 & 0.90 & 0.88 & 0.90 & 0.80 & 0.88 & 0.92 & 0.86 & 0.61 & 0.75 & \\
\hline 0.98 & 0.91 & 0.97 & 0.89 & 0.87 & 0.90 & 0.89 & 0.90 & 0.80 & 0.87 & 0.92 & 0.85 & 0.60 & 0.75 & \\
\hline 0.98 & 0.91 & 0.97 & 0.90 & 0.87 & 0.90 & 0.89 & 0.90 & 0.80 & 0.86 & 0.92 & 0.85 & 0.59 & 0.75 & \\
\hline 0.98 & 0.91 & 0.97 & 0.89 & 0.87 & 0.90 & 0.89 & 0.90 & 0.80 & 0.87 & 0.92 & 0.85 & 0.60 & 0.75 & \\
\hline 0.99 & 0.93 & 0.98 & 0.91 & 0.88 & 0.89 & 0.89 & 0.91 & 0.81 & 0.84 & 0.91 & 0.83 & 0.56 & 0.74 & \\
\hline 0.99 & 0.94 & 0.98 & 0.92 & 0.89 & 0.89 & 0.89 & 0.91 & 0.81 & 0.84 & 0.91 & 0.82 & 0.55 & 0.73 & \\
\hline 0.99 & 0.94 & 0.98 & 0.92 & 0.89 & 0.89 & 0.89 & 0.91 & 0.81 & 0.84 & 0.91 & 0.82 & 0.56 & 0.74 & \\
\hline 0.99 & 0.94 & 0.98 & 0.92 & 0.89 & 0.89 & 0.90 & 0.91 & 0.82 & 0.84 & 91 & 0.83 & 0.56 & 0.73 & \\
\hline 0.99 & 0.93 & 0.98 & 0.91 & 0.88 & 0.89 & 0.89 & 0.91 & 0.81 & 0.85 & 0.91 & 0.83 & 0.57 & 0.74 & \\
\hline 0.99 & 0.93 & 0.98 & 0.92 & 0.89 & 0.89 & 0.90 & 0.91 & 0.81 & 0.85 & 0.91 & 0.83 & 0.56 & 0.73 & \\
\hline 0.99 & 0.95 & 0.98 & 0.93 & 0.91 & 0.90 & 0.91 & 0.92 & 0.84 & 0.84 & 0.92 & 0.82 & 0.53 & 0.71 & \\
\hline 0.97 & 0.89 & 0.96 & 0.88 & 0.86 & 0.91 & 0.89 & 0.90 & 0.81 & 0.89 & 0.93 & 0.87 & 0.59 & 0.72 & \\
\hline 0.98 & 0.93 & 0.97 & 0.92 & 0.90 & 0.90 & 0.90 & 0.91 & 0.83 & 0.86 & 0.92 & 0.84 & 0.54 & 0.71 & \\
\hline 0.96 & 0.98 & 0.95 & 0.96 & 0.96 & 0.89 & 0.93 & 0.92 & 0.87 & 0.77 & 0.88 & 0.77 & 0.43 & 0.64 & \\
\hline 0.96 & 0.98 & 0.96 & 0.96 & 0.96 & 0.88 & 0.93 & 0.92 & 0.87 & 0.77 & 0.87 & 0.76 & 0.43 & 0.64 & \\
\hline 0.96 & 0.97 & 0.95 & 0.96 & 0.95 & 0.89 & 0.93 & 0.92 & 0.87 & 0.77 & 0.88 & 0.77 & 0.44 & 0.64 & \\
\hline 0.96 & 0.98 & 0.95 & 0.96 & 0.95 & 0.89 & 0.93 & 0.92 & 0.87 & 0.77 & 0.88 & 0.77 & 0.44 & 0.64 & \\
\hline 0.96 & 0.97 & 0.96 & 0.95 & 0.95 & 0.89 & 0.93 & 0.92 & 0.87 & 0.78 & 0.88 & 0.77 & 0.44 & 0.64 & \\
\hline 0.96 & 0.97 & 0.96 & 0.96 & 0.95 & 0.89 & 0.93 & 0.92 & 0.88 & 0.78 & 0.88 & 0.77 & 0.44 & 0.64 & \\
\hline 0.96 & 0.97 & 0.96 & 0.95 & 0.95 & 0.89 & 0.93 & 0.92 & 0.87 & 0.78 & 0.88 & 0.78 & 0.45 & 0.64 & \\
\hline 0.96 & 0.97 & 0.96 & 0.96 & 0.95 & 0.89 & 0.93 & 0.92 & 0.88 & 0.78 & 0.88 & 0.77 & 0.44 & 0.64 & \\
\hline 0.96 & 0.97 & 0.96 & 0.96 & 0.95 & 0.89 & 0.93 & 0.92 & 0.88 & 0.78 & 0.88 & 0.77 & 0.44 & 0.64 & \\
\hline 0.96 & 0.97 & 0.96 & 0.95 & 0.95 & 0.89 & 0.93 & 0.92 & 0.88 & 0.78 & 0.88 & 0.77 & 0.44 & 0.64 & \\
\hline 0.96 & 0.97 & 0.96 & 0.96 & 0.95 & 0.89 & 0.93 & 0.92 & 0.88 & 0.78 & 0.88 & 0.77 & 0.44 & 0.64 & \\
\hline 0.96 & 0.98 & 0.96 & 0.96 & 0.96 & 0.89 & 0.93 & 0.92 & 0.88 & 0.77 & 0.88 & 0.77 & 0.43 & 0.64 & \\
\hline 0.96 & 0.98 & 0.96 & 0.96 & 0.96 & 0.89 & 0.93 & 0.92 & 0.87 & 0.78 & 0.88 & 0.77 & 0.44 & 0.64 & \\
\hline 0.96 & 0.97 & 0.96 & 0.96 & 0.95 & 0.89 & 0.93 & 0.92 & 0.87 & 0.78 & 0.88 & 0.77 & 0.45 & 0.65 & \\
\hline 0.97 & 0.97 & 0.96 & 0.96 & 0.95 & 0.89 & 0.93 & 0.92 & 0.87 & 0.79 & 0.89 & 0.78 & 0.45 & 0.65 & \\
\hline 0.97 & 0.97 & 0.96 & 0.96 & 0.95 & 0.89 & 0.93 & 0.92 & 0.87 & 0.79 & 0.88 & 0.78 & 0.45 & 0.65 & \\
\hline 0.98 & 0.96 & 0.97 & 0.95 & 0.94 & 0.91 & 0.93 & 0.93 & 0.87 & 0.83 & 0.91 & 0.82 & 0.49 & 0.68 & \\
\hline 0.97 & 0.96 & 0.97 & 0.95 & 0.94 & 0.91 & 0.93 & 0.93 & 0.87 & 0.82 & 0.90 & 0.81 & 0.48 & 0.67 & \\
\hline 0.97 & 0.97 & 0.96 & 0.95 & 0.95 & 0.90 & 0.93 & 0.92 & 0.87 & 0.80 & 0.90 & 0.80 & 0.47 & 0.66 & \\
\hline 0.97 & 0.96 & 0.96 & 0.95 & 0.94 & 0.90 & 0.93 & 0.92 & 0.87 & 0.81 & 0.90 & 0.80 & 0.48 & 0.66 & \\
\hline 0.97 & 0.97 & 0.96 & 0.96 & 0.95 & 0.90 & 0.93 & 0.92 & 0.87 & 0.80 & 0.89 & 0.79 & 0.46 & 0.66 & \\
\hline 0.97 & 0.96 & 0.96 & 0.95 & 0.95 & 0.90 & 0.93 & 0.92 & 0.87 & 0.80 & 0.89 & 0.79 & 0.47 & 0.66 & \\
\hline 0.97 & 0.97 & 0.96 & 0.96 & 0.95 & 0.90 & 0.93 & 0.92 & 0.87 & 0.79 & 0.89 & 0.78 & 0.46 & 0.65 & \\
\hline
\end{tabular}


Experiment

LEU-COMP-THERM-038-013 LEU-COMP-THERM-038-014 LEU-COMP-THERM-039-001 LEU-COMP-THERM-039-002 LEU-COMP-THERM-039-003 LEU-COMP-THERM-039-004 LEU-COMP-THERM-039-005 LEU-COMP-THERM-039-006 LEU-COMP-THERM-039-007 LEU-COMP-THERM-039-008 LEU-COMP-THERM-039-009 LEU-COMP-THERM-039-010 LEU-COMP-THERM-039-011 LEU-COMP-THERM-039-012 LEU-COMP-THERM-039-013 LEU-COMP-THERM-039-014 LEU-COMP-THERM-039-015 LEU-COMP-THERM-039-016 LEU-COMP-THERM-039-017 LEU-COMP-THERM-040-001_ LEU-COMP-THERM-040-002 LEU-COMP-THERM-040-003 LEU-COMP-THERM-040-004 LEU-COMP-THERM-040-005 LEU-COMP-THERM-040-006 LEU-COMP-THERM-040-007 LEU-COMP-THERM-040-008 LEU-COMP-THERM-040-009 LEU-COMP-THERM-040-010 LEU-COMP-THERM-042-001 LEU-COMP-THERM-042-002 LEU-COMP-THERM-042-003 LEU-COMP-THERM-042-004 LEU-COMP-THERM-042-005 LEU-COMP-THERM-042-006 LEU-COMP-THERM-042-007 LEU-COMP-THERM-043-001_ LEU-COMP-THERM-043-002 LEU-COMP-THERM-043-003 LEU-COMP-THERM-043-004 LEU-COMP-THERM-043-005 LEU-COMP-THERM-043-006

\begin{tabular}{|c|c|c|c|c|c|c|c|c|c|c|c|c|c|c|}
\hline 1 & 2 & 3 & 4 & 5 & 6 & 7 & 8 & 9 & 10 & 11 & 12 & 13 & 14 & 15 \\
\hline 0.97 & 0.97 & 0.96 & 0.95 & 0.95 & 0.90 & 0.93 & 0.92 & 0.87 & 0.80 & 0.89 & 0.79 & 0.46 & 0.65 & \\
\hline 0.97 & 0.97 & 0.96 & 0.96 & 0.96 & 0.89 & 0.93 & 0.92 & 0.87 & 0.78 & 0.88 & 0.78 & 0.45 & 0.65 & \\
\hline 0.99 & 0.98 & 0.98 & 0.95 & 0.94 & 0.88 & 0.91 & 0.92 & 0.85 & 0.79 & 0.89 & 0.78 & 0.48 & 0.69 & \\
\hline 0.99 & 0.98 & 0.98 & 0.95 & 0.94 & 0.88 & 0.92 & 0.92 & 0.85 & 0.79 & 0.89 & 0.78 & 0.48 & 0.69 & \\
\hline 0.98 & 0.98 & 0.98 & 0.96 & 0.95 & 0.88 & 0.92 & 0.92 & 0.85 & 0.78 & 0.88 & 0.77 & 0.46 & 0.68 & \\
\hline 0.98 & 0.98 & 0.98 & 0.96 & 0.95 & 0.88 & 0.92 & 0.92 & 0.86 & 0.78 & 0.88 & 0.77 & 0.46 & 0.68 & \\
\hline 0.97 & 0.98 & 0.96 & 0.96 & 0.96 & 0.89 & 0.92 & 0.92 & 0.87 & 0.78 & 0.88 & 0.77 & 0.44 & 0.65 & \\
\hline 0.97 & 0.98 & 0.97 & 0.96 & 0.96 & 0.88 & 0.92 & 0.92 & 0.87 & 0.77 & 0.88 & 0.77 & 0.44 & 0.66 & \\
\hline 0.99 & 0.98 & 0.98 & 0.96 & 0.94 & 0.88 & 0.92 & 0.92 & 0.85 & 0.78 & 0.88 & 0.77 & 0.47 & 0.68 & \\
\hline 0.98 & 0.98 & 0.98 & 0.96 & 0.95 & 0.88 & 0.92 & 0.92 & 0.85 & 0.78 & 0.88 & 0.77 & 0.46 & .68 & \\
\hline 0.98 & 0.98 & 0.98 & 0.96 & 0.95 & 0.88 & 0.92 & 0.92 & 0.85 & 0.78 & 0.88 & 0.77 & 0.46 & 0.68 & \\
\hline 0.98 & 0.99 & 0.97 & 0.96 & 0.95 & 0.88 & 0.92 & 0.92 & 0.86 & 0.78 & 0.88 & 0.77 & 0.45 & 0.67 & \\
\hline 0.99 & 0.98 & 0.98 & 0.95 & 0.94 & 0.88 & 0.91 & 0.92 & 0.85 & 0.79 & 0.89 & 0.78 & 0.48 & .69 & \\
\hline 0.99 & 0.98 & 0.98 & 0.96 & 0.94 & 0.88 & 0.92 & 0.92 & 0.85 & 0.79 & 0.88 & 0.78 & 0.47 & .69 & \\
\hline 0.99 & 0.98 & 0.98 & 0.96 & 0.94 & 0.88 & 0.92 & 0.92 & 0.85 & 0.79 & 0.88 & 0.78 & 0.47 & 0.69 & \\
\hline 0.99 & 0.98 & 0.98 & 0.96 & 0.94 & 0.88 & 0.92 & 0.92 & 0.85 & 0.78 & 0.88 & 0.78 & 0.47 & 0.68 & \\
\hline 0.98 & 0.98 & 0.98 & 0.96 & 0.95 & 0.88 & 0.92 & 0.92 & 0.85 & 0.78 & 0.88 & 0.77 & 0.46 & 0.68 & \\
\hline 0.99 & 0.98 & 0.98 & 0.96 & 0.94 & 0.88 & 0.92 & 0.92 & 0.85 & 0.78 & 0.88 & 0.78 & 0.47 & 0.68 & \\
\hline 0.99 & 0.98 & 0.98 & 0.96 & 0.94 & 0.88 & 0.92 & 0.92 & 0.85 & 0.78 & 0.88 & 0.78 & 0.47 & 0.68 & \\
\hline 0.98 & 0.96 & 0.98 & 0.94 & 0.94 & 0.92 & 0.94 & 0.93 & 0.88 & 0.85 & 0.92 & 0.84 & 0.52 & 0.69 & \\
\hline 0.98 & 0.94 & 0.97 & 0.94 & 0.93 & 0.92 & 0.94 & 0.93 & 0.88 & 0.86 & 0.93 & 0.85 & 0.53 & 0.70 & \\
\hline 0.98 & 0.95 & 0.97 & 0.94 & 0.93 & 0.92 & 0.94 & 0.93 & 0.88 & 0.86 & 0.93 & 0.85 & 0.53 & 0.70 & \\
\hline 0.98 & 0.95 & 0.98 & 0.94 & 0.93 & 0.92 & 0.94 & 0.93 & 0.88 & 0.86 & 0.93 & 0.84 & 0.53 & 0.70 & \\
\hline 0.98 & 0.95 & 0.98 & 0.93 & 0.92 & 0.91 & 0.93 & 0.92 & 0.86 & 0.85 & 0.92 & 0.84 & 0.52 & 0.69 & \\
\hline 0.98 & 0.94 & 0.97 & 0.92 & 0.91 & 0.92 & 0.92 & 0.92 & 0.86 & 0.86 & 0.93 & 0.85 & 0.54 & 0.70 & \\
\hline 0.98 & 0.94 & 0.98 & 0.92 & 0.92 & 0.92 & 0.92 & 0.92 & 0.86 & 0.86 & 0.92 & 0.84 & 0.53 & 0.70 & \\
\hline 0.98 & 0.94 & 0.98 & 0.93 & 0.92 & 0.91 & 0.92 & 0.92 & 0.86 & 0.85 & 0.92 & 0.84 & 0.53 & 0.70 & \\
\hline 0.97 & 0.92 & 0.96 & 0.92 & 0.91 & 0.93 & 0.93 & 0.93 & 0.87 & 0.88 & 0.94 & 0.86 & 0.55 & 0.71 & \\
\hline 0.98 & 0.94 & 0.97 & 0.92 & 0.91 & 0.92 & 0.93 & 0.92 & 0.86 & 0.86 & 0.93 & 0.85 & 0.54 & 0.70 & \\
\hline 0.91 & 0.79 & 0.89 & 0.82 & 0.77 & 0.91 & 0.85 & 0.88 & 0.77 & 0.92 & 0.92 & 0.89 & 0.68 & 0.76 & \\
\hline 0.90 & 0.77 & 0.88 & 0.80 & 0.75 & 0.89 & 0.84 & 0.86 & 0.75 & 0.92 & 0.91 & 0.88 & 0.68 & 0.76 & \\
\hline 0.90 & 0.76 & 0.88 & 0.79 & 0.74 & 0.89 & 0.83 & 0.85 & 0.75 & 0.92 & 0.91 & 0.88 & 0.69 & 0.76 & \\
\hline 0.89 & 0.76 & 0.88 & 0.78 & 0.74 & 0.89 & 0.83 & 0.85 & 0.74 & 0.92 & 0.91 & 0.88 & 0.69 & 0.76 & \\
\hline 0.90 & 0.76 & 0.88 & 0.79 & 0.74 & 0.89 & 0.83 & 0.85 & 0.74 & 0.92 & 0.91 & 0.88 & 0.69 & 0.76 & \\
\hline 0.90 & 0.78 & 0.89 & 0.80 & 0.76 & 0.89 & 0.84 & 0.86 & 0.75 & 0.91 & 0.91 & 0.88 & 0.67 & 0.75 & \\
\hline 0.90 & 0.77 & 0.88 & 0.79 & 0.75 & 0.89 & 0.83 & 0.86 & 0.75 & 0.91 & 0.91 & 0.88 & 0.68 & 0.75 & \\
\hline 0.86 & 0.81 & 0.86 & 0.90 & 0.88 & 0.98 & 0.96 & 0.97 & 0.95 & 0.94 & 0.96 & 0.92 & 0.63 & 0.73 & \\
\hline 0.86 & 0.80 & 0.85 & 0.89 & 0.87 & 0.98 & 0.96 & 0.96 & 0.95 & 0.94 & 0.96 & 0.93 & 0.64 & 0.73 & \\
\hline 0.86 & 0.81 & 0.86 & 0.90 & 0.88 & 0.98 & 0.96 & 0.97 & 0.95 & 0.94 & 0.96 & 0.93 & 0.63 & 0.73 & \\
\hline 0.86 & 0.80 & 0.86 & 0.89 & 0.87 & 0.98 & 0.96 & 0.96 & 0.95 & 0.94 & 0.96 & 0.93 & 0.64 & 0.73 & \\
\hline 0.86 & 0.80 & 0.86 & 0.89 & 0.87 & 0.98 & 0.96 & 0.96 & 0.95 & 0.94 & 0.96 & 0.93 & 0.64 & 0.73 & \\
\hline 0.85 & 0.80 & 0.85 & 0.89 & 0.87 & 0.98 & 0.95 & 0.96 & 0.94 & 0.94 & 0.96 & 0.93 & 0.64 & 0.73 & \\
\hline
\end{tabular}


Experiment

LEU-COMP-THERM-043-007 LEU-COMP-THERM-043-008 LEU-COMP-THERM-043-009 LEU-COMP-THERM-044-001 LEU-COMP-THERM-044-002 LEU-COMP-THERM-044-003 LEU-COMP-THERM-044-004 LEU-COMP-THERM-044-005 LEU-COMP-THERM-044-006 LEU-COMP-THERM-044-007 LEU-COMP-THERM-044-008 LEU-COMP-THERM-044-009 LEU-COMP-THERM-044-010 LEU-COMP-THERM-045-001_ LEU-COMP-THERM-045-002 LEU-COMP-THERM-045-003 LEU-COMP-THERM-045-004 LEU-COMP-THERM-045-005 LEU-COMP-THERM-045-006 LEU-COMP-THERM-045-007 EU-COMP-THERM-045-008 LEU-COMP-THERM-045-009 LEU-COMP-THERM-045-010 EU-COMP-THERM-045-011 LEU-COMP-THERM-045-012 LEU-COMP-THERM-045-013 LEU-COMP-THERM-045-014 LEU-COMP-THERM-045-015 LEU-COMP-THERM-045-016 LEU-COMP-THERM-045-017 LEU-COMP-THERM-045-018 LEU-COMP-THERM-045-019 LEU-COMP-THERM-045-020 LEU-COMP-THERM-045-021 LEU-COMP-THERM-046-001 LEU-COMP-THERM-046-002 LEU-COMP-THERM-046-003 LEU-COMP-THERM-046-004 LEU-COMP-THERM-046-005 LEU-COMP-THERM-046-006 LEU-COMP-THERM-046-007 LEU-COMP-THERM-046-008_

\begin{tabular}{|c|c|c|c|c|c|c|c|c|c|c|c|c|c|c|}
\hline 1 & 2 & 3 & 4 & 5 & 6 & 7 & 8 & 9 & 10 & 11 & 12 & 13 & 14 & 15 \\
\hline 0.86 & 0.81 & 0.86 & 0.89 & 0.87 & 0.98 & 0.95 & 0.96 & 0.94 & 0.94 & 0.96 & 0.92 & 0.64 & 0.73 & 0.56 \\
\hline 0.87 & 0.82 & 0.86 & 0.90 & 0.88 & 0.98 & 0.96 & 0.97 & 0.95 & 0.93 & 0.96 & 0.92 & 0.63 & 0.73 & 0.57 \\
\hline 0.86 & 0.81 & 0.86 & 0.90 & 0.87 & 0.98 & 0.95 & 0.96 & 0.94 & 0.94 & 0.96 & 0.92 & 0.63 & 0.73 & 0.56 \\
\hline 0.86 & 0.82 & 0.86 & 0.90 & 0.88 & 0.98 & 0.95 & 0.96 & 0.94 & 0.93 & 0.95 & 0.91 & 0.62 & 0.72 & 0.56 \\
\hline 0.87 & 0.82 & 0.86 & 0.90 & 0.88 & 0.98 & 0.96 & 0.96 & 0.94 & 0.93 & 0.96 & 0.92 & 0.63 & 0.73 & 0.56 \\
\hline 0.87 & 0.82 & 0.86 & 0.90 & 0.89 & 0.98 & 0.96 & 0.96 & 0.95 & 0.92 & 0.95 & 0.91 & 0.62 & 0.72 & 0.56 \\
\hline 0.86 & 0.81 & 0.86 & 0.89 & 0.87 & 0.98 & 0.95 & 0.96 & 0.94 & 0.93 & 0.96 & 0.92 & 0.63 & 0.72 & 0.56 \\
\hline 0.86 & 0.81 & 0.86 & 0.89 & 0.87 & 0.98 & 0.95 & 0.96 & 0.94 & 0.93 & 0.95 & 0.92 & 0.63 & 0.72 & 0.56 \\
\hline 0.86 & 0.81 & 0.86 & 0.90 & 0.88 & 0.98 & 0.95 & 0.96 & 0.94 & 0.93 & 0.95 & 0.91 & 0.62 & 0.72 & 0.56 \\
\hline 0.86 & 0.81 & 0.86 & 0.90 & 0.88 & 0.98 & 0.95 & 0.96 & 0.94 & 0.93 & 0.95 & 0.91 & 0.63 & 0.72 & 0.56 \\
\hline 0.86 & 0.81 & 0.86 & 0.89 & 0.87 & 0.98 & 0.95 & 0.96 & 0.94 & 0.93 & 0.95 & 0.92 & 0.63 & 0.72 & 0.56 \\
\hline 0.87 & 0.82 & 0.86 & 0.90 & 0.89 & 0.98 & 0.96 & 0.97 & 0.95 & 0.93 & 0.95 & 0.91 & 0.62 & 0.72 & 0.56 \\
\hline 0.86 & 0.81 & 0.85 & 0.89 & 0.87 & 0.98 & 0.95 & 0.96 & 0.94 & 0.93 & 0.95 & 0.92 & 0.63 & 0.72 & 0.56 \\
\hline 0.97 & 0.89 & 0.96 & 0.87 & 0.84 & 0.87 & 0.85 & 0.88 & 0.77 & 0.85 & 0.90 & 0.84 & 0.60 & 0.76 & 0.64 \\
\hline 0.97 & 0.88 & 0.96 & 0.86 & 0.83 & 0.87 & 0.85 & 0.88 & 0.77 & 0.86 & 0.90 & 0.84 & 0.60 & 0.76 & 0.64 \\
\hline 0.88 & 0.74 & 0.87 & 0.71 & 0.67 & 0.78 & 0.73 & 0.78 & 0.64 & 0.82 & 0.83 & 0.81 & 0.73 & 0.81 & 0.67 \\
\hline 0.87 & 0.74 & 0.87 & 0.71 & 0.66 & 0.77 & 0.73 & 0.77 & 0.63 & 0.82 & 0.83 & 0.81 & 0.73 & 0.81 & 0.67 \\
\hline 0.92 & 0.81 & 0.92 & 0.78 & 0.74 & 0.82 & 0.78 & 0.82 & 0.69 & 0.84 & 0.87 & 0.83 & 0.68 & 0.79 & 0.66 \\
\hline 0.94 & 0.83 & 0.93 & 0.82 & 0.79 & 0.86 & 0.82 & 0.85 & 0.74 & 0.87 & 0.90 & 0.85 & 0.64 & 0.77 & 0.63 \\
\hline 0.82 & 0.72 & 0.82 & 0.80 & 0.76 & 0.93 & 0.87 & 0.90 & 0.85 & 0.94 & 0.93 & 0.92 & 0.72 & 0.78 & 0.60 \\
\hline 0.82 & 0.71 & 0.82 & 0.79 & 0.76 & 0.93 & 0.86 & 0.90 & 0.84 & 0.94 & 0.93 & 0.92 & 0.73 & 0.78 & 0.60 \\
\hline 0.92 & 0.81 & 0.91 & 0.85 & 0.82 & 0.93 & 0.89 & 0.92 & 0.84 & 0.94 & 0.95 & 0.92 & 0.70 & 0.80 & 0.64 \\
\hline 0.81 & 0.68 & 0.81 & 0.74 & 0.69 & 0.88 & 0.81 & 0.85 & 0.77 & 0.92 & 0.90 & 0.91 & 0.80 & 0.83 & 0.65 \\
\hline 0.96 & 0.87 & 0.95 & 0.85 & 0.82 & 0.87 & 0.84 & 0.87 & 0.76 & 0.87 & 0.91 & 0.85 & 0.62 & 0.76 & 0.63 \\
\hline 0.96 & 0.87 & 0.95 & 0.85 & 0.82 & 0.87 & 0.85 & 0.87 & 0.76 & 0.86 & 0.91 & 0.85 & 0.62 & 0.76 & 0.63 \\
\hline 0.96 & 0.87 & 0.95 & 0.84 & 0.81 & 0.87 & 0.85 & 0.88 & 0.77 & 0.87 & 0.91 & 0.86 & 0.63 & 0.77 & 0.64 \\
\hline 0.96 & 0.86 & 0.95 & 0.84 & 0.81 & 0.87 & 0.85 & 0.87 & 0.77 & 0.87 & 0.91 & 0.86 & 0.64 & 0.77 & 0.63 \\
\hline 0.81 & 0.66 & 0.81 & 0.62 & 0.58 & 0.74 & 0.68 & 0.71 & 0.59 & 0.81 & 0.80 & 0.80 & 0.79 & 0.81 & 0.65 \\
\hline 0.80 & 0.64 & 0.80 & 0.61 & 0.57 & 0.74 & 0.67 & 0.71 & 0.58 & 0.81 & 0.79 & 0.80 & 0.80 & 0.81 & 0.65 \\
\hline 0.82 & 0.67 & 0.82 & 0.64 & 0.60 & 0.76 & 0.69 & 0.73 & 0.61 & 0.84 & 0.82 & 0.82 & 0.79 & 0.81 & 0.65 \\
\hline 0.93 & 0.82 & 0.92 & 0.79 & 0.76 & 0.86 & 0.82 & 0.85 & 0.74 & 0.88 & 0.90 & 0.87 & 0.67 & 0.77 & 0.62 \\
\hline 0.93 & 0.81 & 0.92 & 0.79 & 0.76 & 0.86 & 0.81 & 0.84 & 0.73 & 0.88 & 0.90 & 0.86 & 0.67 & 0.77 & 0.62 \\
\hline 0.81 & 0.69 & 0.80 & 0.77 & 0.73 & 0.92 & 0.85 & 0.88 & 0.83 & 0.94 & 0.92 & 0.93 & 0.75 & 0.78 & 0.59 \\
\hline 0.81 & 0.69 & 0.80 & 0.76 & 0.73 & 0.92 & 0.85 & 0.88 & 0.83 & 0.94 & 0.92 & 0.93 & 0.75 & 0.78 & 0.59 \\
\hline 0.88 & 0.84 & 0.87 & 0.92 & 0.90 & 0.98 & 0.97 & 0.97 & 0.95 & 0.92 & 0.95 & 0.90 & 0.60 & 0.72 & 0.57 \\
\hline 0.88 & 0.84 & 0.87 & 0.92 & 0.90 & 0.98 & 0.97 & 0.97 & 0.95 & 0.92 & 0.95 & 0.90 & 0.60 & 0.72 & 0.57 \\
\hline 0.88 & 0.84 & 0.88 & 0.92 & 0.90 & 0.98 & 0.97 & 0.97 & 0.95 & 0.91 & 0.95 & 0.90 & 0.60 & 0.72 & 0.57 \\
\hline 0.88 & 0.84 & 0.87 & 0.92 & 0.90 & 0.98 & 0.97 & 0.97 & 0.95 & 0.92 & 0.95 & 0.90 & 0.60 & 0.72 & 0.57 \\
\hline 0.88 & 0.84 & 0.87 & 0.92 & 0.90 & 0.98 & 0.96 & 0.97 & 0.95 & 0.92 & 0.95 & 0.90 & 0.61 & 0.72 & 0.57 \\
\hline 0.88 & 0.84 & 0.87 & 0.92 & 0.90 & 0.98 & 0.96 & 0.97 & 0.95 & 0.92 & 0.95 & 0.90 & 0.61 & 0.72 & 0.57 \\
\hline 0.88 & 0.84 & 0.87 & 0.92 & 0.90 & 0.98 & 0.96 & 0.97 & 0.95 & 0.92 & 0.95 & 0.90 & 0.61 & 0.72 & 0.57 \\
\hline 0.88 & 0.84 & 0.87 & 0.92 & 0.90 & 0.98 & 0.97 & 0.97 & 0.95 & 0.92 & 0.95 & 0.90 & 0.60 & 0.72 & 0.57 \\
\hline
\end{tabular}


1002

1003

1004

1005

1006

1007

1008
Experiment

LEU-COMP-THERM-046-009 LEU-COMP-THERM-046-010 LEU-COMP-THERM-046-011 LEU-COMP-THERM-046-012 LEU-COMP-THERM-046-013 LEU-COMP-THERM-046-014 LEU-COMP-THERM-046-015 LEU-COMP-THERM-046-016 LEU-COMP-THERM-046-017 LEU-COMP-THERM-046-018 LEU-COMP-THERM-046-019 LEU-COMP-THERM-046-020 LEU-COMP-THERM-046-021 LEU-COMP-THERM-046-022 LEU-COMP-THERM-047-001 LEU-COMP-THERM-047-002 LEU-COMP-THERM-047-003 LEU-COMP-THERM-048-001 LEU-COMP-THERM-048-002 LEU-COMP-THERM-048-003 LEU-COMP-THERM-048-004 LEU-COMP-THERM-048-005 LEU-COMP-THERM-049-001 LEU-COMP-THERM-049-002 LEU-COMP-THERM-049-003 LEU-COMP-THERM-049-004 LEU-COMP-THERM-049-005 LEU-COMP-THERM-049-006 LEU-COMP-THERM-049-007 LEU-COMP-THERM-049-008 LEU-COMP-THERM-049-009 LEU-COMP-THERM-049-010 LEU-COMP-THERM-049-011 LEU-COMP-THERM-049-012 LEU-COMP-THERM-049-013 LEU-COMP-THERM-049-014 LEU-COMP-THERM-049-015 LEU-COMP-THERM-049-016 LEU-COMP-THERM-049-017 LEU-COMP-THERM-049-018 LEU-COMP-THERM-050-001 LEU-COMP-THERM-050-002_-

\begin{tabular}{|c|c|c|c|c|c|c|c|c|c|c|c|c|c|c|}
\hline 1 & 2 & 3 & 4 & 5 & 6 & 7 & 8 & 9 & 10 & 11 & 12 & 13 & 14 & 15 \\
\hline 0.88 & 0.84 & 0.87 & 0.92 & 0.90 & 0.98 & 0.96 & 0.97 & 0.95 & 0.92 & 0.95 & 0.90 & 0.61 & 0.72 & 0.5 \\
\hline 0.88 & 0.84 & 0.88 & 0.92 & 0.90 & 0.98 & 0.97 & 0.97 & 0.95 & 0.91 & 0.95 & 0.90 & 0.60 & 0.72 & \\
\hline 0.88 & 0.84 & 0.88 & 0.92 & 0.90 & 0.98 & 0.96 & 0.97 & 0.95 & 0.92 & 0.95 & 0.90 & 0.61 & 0.72 & \\
\hline 0.87 & 0.83 & 0.87 & 0.91 & 0.89 & 0.98 & 0.96 & 0.97 & 0.95 & 0.93 & 0.96 & 0.91 & 0.62 & 0.73 & \\
\hline 0.87 & 0.83 & 0.87 & 0.91 & 0.89 & 0.98 & 0.96 & 0.97 & 0.95 & 0.93 & 0.96 & 0.91 & 0.62 & 0.73 & \\
\hline 0.88 & 0.83 & 0.87 & 0.91 & 0.89 & 0.98 & 0.96 & 0.97 & 0.95 & 0.93 & 0.96 & 0.91 & 0.62 & 0.73 & \\
\hline 0.87 & 0.83 & 0.87 & 0.91 & 0.89 & 0.98 & 0.96 & 0.97 & 0.95 & 0.93 & 0.96 & 0.92 & 0.62 & 0.73 & \\
\hline 0.88 & 0.83 & 0.87 & 0.91 & 0.89 & 0.98 & 0.96 & 0.97 & 0.95 & 0.93 & 0.96 & 0.91 & 0.62 & 0.73 & \\
\hline 0.88 & 0.83 & 0.87 & 0.91 & 0.89 & 0.98 & 0.96 & 0.97 & 0.95 & 0.93 & 0.96 & 0.91 & 0.62 & 0.73 & \\
\hline 0.87 & 0.83 & 0.87 & 0.91 & 0.89 & 0.98 & 0.96 & 0.97 & 0.95 & 0.92 & 0.95 & 0.91 & 0.61 & 0.72 & \\
\hline 0.87 & 0.83 & 0.87 & 0.91 & 0.89 & 0.98 & 0.96 & 0.97 & 0.95 & 0.92 & 0.95 & 0.91 & 0.61 & 0.72 & \\
\hline 0.87 & 0.83 & 0.87 & 0.91 & 0.89 & 0.98 & 0.96 & 0.97 & 0.95 & 0.92 & 0.95 & 0.91 & 0.61 & 0.72 & \\
\hline 0.87 & 0.83 & 0.87 & 0.91 & 0.89 & 0.98 & 0.96 & 0.97 & 0.95 & 0.92 & 0.95 & 0.91 & 0.62 & 0.72 & \\
\hline 0.87 & 0.83 & 0.87 & 0.91 & 0.89 & 0.98 & 0.96 & 0.97 & 0.95 & .92 & 0.95 & 0.91 & 0.61 & 0.72 & \\
\hline 0.84 & 0.69 & 0.82 & 0.75 & 0.71 & 0.92 & 0.84 & 0.85 & 0.79 & .97 & 0.94 & 0.94 & 0.73 & 0.75 & \\
\hline 0.94 & 0.87 & 0.94 & 0.91 & 0.89 & 0.98 & 0.95 & 0.96 & 0.91 & 0.95 & 0.98 & 0.94 & 0.65 & 0.75 & \\
\hline 0.91 & 0.81 & 0.91 & 0.86 & 0.84 & 0.97 & 0.92 & 0.93 & 0.88 & 0.97 & 0.98 & 0.95 & 0.68 & 0.76 & \\
\hline 0.86 & 0.72 & 0.85 & 0.75 & 0.70 & 0.87 & 0.80 & 0.84 & 0.73 & 0.91 & 0.89 & 0.88 & 0.76 & 0.81 & \\
\hline 0.86 & 0.72 & 0.85 & 0.75 & 0.70 & 0.87 & 0.80 & 84 & 73 & 0.91 & 0.89 & 0.88 & 0.76 & 0.81 & \\
\hline 0.86 & 0.72 & 0.84 & 0.75 & 0.70 & 0.87 & 0.80 & 0.84 & 0.73 & 0.90 & 0.89 & 0.88 & 0.76 & 0.81 & \\
\hline 0.86 & 0.72 & 0.84 & 0.75 & 0.70 & 0.87 & 0.80 & 0.84 & 0.73 & 0.91 & 0.89 & 0.88 & 0.76 & 0.81 & \\
\hline 0.86 & 0.72 & 0.85 & 0.75 & 0.70 & 0.87 & 0.80 & 0.84 & 0.73 & 0.90 & 0.89 & 0.88 & 0.76 & 0.81 & \\
\hline 0.78 & 0.62 & 0.78 & 0.59 & 0.55 & 0.71 & 0.65 & 0.68 & 0.55 & 0.79 & 0.77 & 0.78 & 0.81 & 0.82 & \\
\hline 0.78 & 0.62 & 0.78 & 0.60 & 0.56 & 0.71 & 0.65 & 0.68 & 0.55 & 0.79 & 0.77 & 0.78 & 0.80 & 0.82 & \\
\hline 0.76 & 0.59 & 0.76 & 0.57 & 0.53 & 0.70 & 0.63 & 0.66 & 0.54 & 0.79 & 0.76 & 0.77 & 0.82 & 0.81 & \\
\hline 0.75 & 0.58 & 0.75 & 0.56 & 0.52 & 0.69 & 0.62 & 0.65 & 0.52 & 0.78 & 0.75 & 0.77 & 0.82 & 0.81 & \\
\hline 0.88 & 0.76 & 0.88 & 0.73 & 0.69 & 0.79 & 0.75 & 0.79 & 0.66 & 0.82 & 0.84 & 0.81 & 0.73 & 0.82 & \\
\hline 0.88 & 0.75 & 0.87 & 0.73 & 0.69 & 0.79 & 0.75 & 0.78 & 0.65 & 0.8 & 0.84 & 0.81 & 0.74 & 0.82 & \\
\hline 0.88 & 0.77 & 0.88 & 0.74 & 0.70 & 0.79 & 0.76 & 0.79 & 0.66 & 0.82 & 0.84 & 0.81 & 0.73 & 0.82 & \\
\hline 0.87 & 0.74 & 0.86 & 0.71 & 0.67 & 0.78 & 0.74 & 0.77 & 0.64 & 0.83 & 0.84 & 0.81 & 0.75 & 0.82 & \\
\hline 0.93 & 0.83 & 0.92 & 0.80 & 0.77 & 0.83 & 0.81 & 0.84 & 0.72 & 0.83 & 0.87 & 0.82 & 0.68 & 0.80 & \\
\hline 0.93 & 0.83 & 0.92 & 0.81 & 0.77 & 0.83 & 0.81 & 0.84 & 0.72 & 0.83 & 0.87 & 0.82 & 0.68 & 0.80 & \\
\hline 0.93 & 0.83 & 0.92 & 0.81 & 0.77 & 0.83 & 0.81 & 0.84 & 0.72 & 0.83 & 0.87 & 0.82 & 0.68 & 0.80 & \\
\hline 0.92 & 0.81 & 0.92 & 0.79 & 0.75 & 0.83 & 0.80 & 0.83 & 0.71 & 0.84 & 0.87 & 0.82 & 0.70 & 0.81 & \\
\hline 0.88 & 0.76 & 0.87 & 0.73 & 0.69 & 0.78 & 0.74 & 0.78 & 0.65 & 0.81 & 0.83 & 0.80 & 0.73 & 0.81 & \\
\hline 0.87 & 0.74 & 0.87 & 0.71 & 0.67 & 0.78 & 0.73 & 0.77 & 0.64 & 0.82 & 0.83 & 0.80 & 0.74 & 0.82 & \\
\hline 0.87 & 0.75 & 0.87 & 0.72 & 0.68 & 0.78 & 0.74 & 0.78 & 0.64 & 0.82 & 0.83 & 0.80 & 0.73 & 0.82 & \\
\hline 0.89 & 0.77 & 0.88 & 0.74 & 0.70 & 0.80 & 0.76 & 0.79 & 0.66 & 0.82 & 0.84 & 0.81 & 0.73 & 0.82 & \\
\hline 0.87 & 0.74 & 0.87 & 0.71 & 0.68 & 0.79 & 0.74 & 0.77 & 0.65 & 0.83 & 0.84 & 0.81 & 0.75 & 0.82 & \\
\hline 0.83 & 0.68 & 0.83 & 0.66 & 0.63 & 0.77 & 0.71 & 0.74 & 0.62 & 0.84 & 0.83 & 0.83 & 0.78 & 0.81 & \\
\hline 0.99 & 0.98 & 0.98 & 0.95 & 0.94 & 0.89 & 0.92 & 0.92 & 0.85 & 0.79 & 0.89 & 0.79 & 0.48 & 0.68 & \\
\hline 0.98 & 0.98 & 0.98 & 0.95 & 0.94 & 0.89 & 0.92 & 0.92 & 0.85 & 0.80 & 0.89 & 0.79 & 0.47 & 0.68 & \\
\hline
\end{tabular}


Case

1009

1010

1011

1012

1013

1014

1015

1016

1017

1018

1019

1020

1021

1022

1023

1024

1025

1026

1027

1028

1029

1030

1031

1032

1033

1034

1035

1036

1037

1038

1039

1040

1041

1042

1043

1044

1045

1046

1047

1048

1049

1050
Experiment

LEU-COMP-THERM-050-003 LEU-COMP-THERM-050-004 LEU-COMP-THERM-050-005 LEU-COMP-THERM-050-006 LEU-COMP-THERM-050-007 LEU-COMP-THERM-050-008 LEU-COMP-THERM-050-009 LEU-COMP-THERM-050-010 LEU-COMP-THERM-050-011 LEU-COMP-THERM-050-012 LEU-COMP-THERM-050-013 LEU-COMP-THERM-050-014 LEU-COMP-THERM-050-015 LEU-COMP-THERM-050-016 LEU-COMP-THERM-050-017 LEU-COMP-THERM-050-018 LEU-COMP-THERM-051-001_ LEU-COMP-THERM-051-002 LEU-COMP-THERM-051-003 LEU-COMP-THERM-051-004 LEU-COMP-THERM-051-005 LEU-COMP-THERM-051-006 LEU-COMP-THERM-051-007 LEU-COMP-THERM-051-008 LEU-COMP-THERM-051-009 LEU-COMP-THERM-051-010 LEU-COMP-THERM-051-011 LEU-COMP-THERM-051-012 LEU-COMP-THERM-051-013 LEU-COMP-THERM-051-014 LEU-COMP-THERM-051-015 LEU-COMP-THERM-051-016 LEU-COMP-THERM-051-017 LEU-COMP-THERM-051-018 LEU-COMP-THERM-051-019 LEU-COMP-THERM-052-001 LEU-COMP-THERM-052-002 LEU-COMP-THERM-052-003 LEU-COMP-THERM-052-004 LEU-COMP-THERM-052-005 LEU-COMP-THERM-052-006 LEU-COMP-THERM-053-001_-

\begin{tabular}{|c|c|c|c|c|c|c|c|c|c|c|c|c|c|c|}
\hline 1 & 2 & 3 & 4 & 5 & 6 & 7 & 8 & 9 & 10 & 11 & 12 & 13 & 14 & 15 \\
\hline 0.99 & 0.97 & 0.98 & 0.95 & 0.94 & 0.89 & 0.92 & 0.93 & 0.85 & 0.81 & 0.90 & 0.80 & 0.49 & 0.70 & 0.60 \\
\hline 0.99 & 0.97 & 0.98 & 0.95 & 0.93 & 0.89 & 0.92 & 0.93 & 0.85 & 0.81 & 0.90 & 0.80 & 0.50 & 0.70 & 0.60 \\
\hline 0.99 & 0.97 & 0.99 & 0.94 & 0.93 & 0.90 & 0.92 & 0.93 & 0.85 & 0.82 & 0.91 & 0.81 & 0.51 & 0.71 & 0.61 \\
\hline 0.99 & 0.97 & 0.99 & 0.94 & 0.93 & 0.90 & 0.92 & 0.93 & 0.85 & 0.82 & 0.91 & 0.81 & 0.52 & 0.71 & 0.61 \\
\hline 0.99 & 0.96 & 0.99 & 0.94 & 0.93 & 0.90 & 0.92 & 0.93 & 0.85 & 0.82 & 0.91 & 0.81 & 0.52 & 0.71 & 0.61 \\
\hline 0.99 & 0.97 & 0.98 & 0.95 & 0.94 & 0.89 & 0.92 & 0.93 & 0.85 & 0.80 & 0.90 & 0.79 & 0.49 & 0.69 & 0.60 \\
\hline 0.99 & 0.97 & 0.98 & 0.95 & 0.94 & 0.89 & 0.92 & 0.93 & 0.85 & 0.81 & 0.90 & 0.80 & 0.49 & 0.69 & 0.60 \\
\hline 0.99 & 0.97 & 0.98 & 0.95 & 0.94 & 0.89 & 0.92 & 0.92 & 0.85 & 0.81 & 0.90 & 0.80 & 0.49 & 0.69 & 0.60 \\
\hline 0.99 & 0.97 & 0.98 & 0.95 & 0.93 & 0.89 & 0.92 & 0.93 & 0.85 & 0.81 & 0.90 & 0.80 & 0.50 & 0.70 & 0.61 \\
\hline 0.99 & 0.97 & 0.98 & 0.95 & 0.93 & 0.90 & 0.92 & 0.93 & 0.85 & 0.82 & 0.90 & 0.81 & 0.50 & 0.70 & 0.60 \\
\hline 0.99 & 0.97 & 0.98 & 0.95 & 0.93 & 0.90 & 0.92 & 0.93 & 0.85 & 0.82 & 0.90 & 0.81 & 0.50 & 0.70 & 0.60 \\
\hline 0.99 & 0.97 & 0.98 & 0.94 & 0.93 & 0.90 & 0.92 & 0.93 & 0.85 & 0.82 & 0.91 & 0.81 & 0.52 & 0.71 & 0.61 \\
\hline 0.99 & 0.96 & 0.98 & 0.94 & 0.93 & 0.90 & 0.92 & 0.93 & 0.85 & 0.82 & 0.91 & 0.81 & 0.52 & 0.71 & 0.61 \\
\hline 0.99 & 0.97 & 0.98 & 0.94 & 0.93 & 0.90 & 0.92 & 0.93 & 0.85 & 0.82 & 0.91 & 0.81 & 0.51 & 0.71 & 0.61 \\
\hline 0.99 & 0.96 & 0.98 & 0.94 & 0.93 & 0.90 & 0.92 & 0.93 & 0.85 & 0.82 & 0.91 & 0.81 & 0.52 & 0.71 & 0.61 \\
\hline 0.99 & 0.97 & 0.98 & 0.94 & 0.93 & 0.90 & 0.92 & 0.93 & 0.85 & 0.82 & 0.91 & 0.81 & 0.52 & 0.71 & 0.61 \\
\hline 0.83 & 0.67 & 0.81 & 0.68 & 0.64 & 0.81 & 0.73 & 0.74 & 0.64 & 0.88 & 0.85 & 0.84 & 0.66 & 0.69 & 0.49 \\
\hline 0.79 & 0.61 & 0.77 & 0.65 & 0.61 & 0.86 & 0.75 & 0.77 & 0.69 & 0.94 & 0.88 & 0.90 & 0.76 & 0.74 & 0.50 \\
\hline 0.79 & 0.62 & 0.78 & 0.66 & 0.62 & 0.86 & 0.76 & 0.78 & 0.69 & 0.94 & 0.89 & 0.90 & 0.76 & 0.74 & 0.50 \\
\hline 0.80 & 0.62 & 0.78 & 0.67 & 0.62 & 0.86 & 0.76 & 0.78 & 0.69 & 0.94 & 0.89 & 0.90 & 0.76 & 0.74 & 0.51 \\
\hline 0.81 & 0.64 & 0.79 & 0.68 & 0.64 & 0.87 & 0.77 & 0.79 & 0.71 & 0.94 & 0.89 & 0.91 & 0.76 & 0.75 & 0.51 \\
\hline 0.81 & 0.64 & 0.79 & 0.68 & 0.64 & 0.87 & 0.77 & 0.79 & 0.70 & 0.94 & 0.89 & 0.91 & 0.76 & 0.75 & 0.51 \\
\hline 0.80 & 0.63 & 0.79 & 0.67 & 0.63 & 0.87 & 0.77 & 0.79 & 0.70 & 0.94 & 0.89 & 0.91 & 0.76 & 0.74 & 0.51 \\
\hline 0.82 & 0.66 & 0.81 & 0.70 & 0.66 & 0.88 & 0.79 & 0.81 & 0.72 & 0.95 & 0.90 & 0.91 & 0.75 & 0.75 & 0.52 \\
\hline 0.81 & 0.64 & 0.79 & 0.69 & 0.65 & 0.87 & 0.78 & 0.79 & 0.71 & 0.94 & 0.89 & 0.91 & 0.75 & 0.74 & 0.50 \\
\hline 0.82 & 0.65 & 0.80 & 0.65 & 0.61 & 0.80 & 0.71 & 0.73 & 0.62 & 0.88 & 0.84 & 0.84 & 0.71 & 0.72 & 0.50 \\
\hline 0.82 & 0.64 & 0.80 & 0.65 & 0.61 & 0.80 & 0.71 & 0.73 & 0.62 & 0.88 & 0.84 & 0.84 & 0.71 & 0.71 & 0.50 \\
\hline 0.82 & 0.64 & 0.80 & 0.64 & 0.61 & 0.80 & 0.71 & 0.73 & 0.62 & 0.88 & 0.84 & 0.85 & 0.71 & 0.71 & 0.50 \\
\hline 0.81 & 0.64 & 0.80 & 0.64 & 0.60 & 0.80 & 0.71 & 0.72 & 0.61 & 0.88 & 0.84 & 0.85 & 0.71 & 0.71 & 0.49 \\
\hline 0.83 & 0.66 & 0.81 & 0.66 & 0.62 & 0.81 & 0.72 & 0.74 & 0.63 & 0.89 & 0.85 & 0.85 & 0.70 & 0.71 & 0.49 \\
\hline 0.81 & 0.63 & 0.79 & 0.63 & 0.59 & 0.79 & 0.70 & 0.72 & 0.61 & 0.88 & 0.84 & 0.84 & 0.71 & 0.71 & 0.49 \\
\hline 0.82 & 0.65 & 0.81 & 0.66 & 0.62 & 0.81 & 0.72 & 0.73 & 0.63 & 0.88 & 0.85 & 0.85 & 0.70 & 0.71 & 0.49 \\
\hline 0.81 & 0.63 & 0.79 & 0.63 & 0.59 & 0.79 & 0.70 & 0.72 & 0.61 & 0.88 & 0.84 & 0.84 & 0.71 & 0.71 & 0.49 \\
\hline 0.82 & 0.65 & 0.80 & 0.65 & 0.61 & 0.80 & 0.71 & 0.73 & 0.62 & 0.88 & 0.85 & 0.85 & 0.69 & 0.70 & 0.49 \\
\hline 0.84 & 0.68 & 0.82 & 0.69 & 0.65 & 0.82 & 0.74 & 0.75 & 0.65 & 0.88 & 0.86 & 0.84 & 0.67 & 0.70 & 0.49 \\
\hline 0.78 & 0.72 & 0.78 & 0.70 & 0.67 & 0.71 & 0.70 & 0.72 & 0.63 & 0.75 & 0.78 & 0.79 & 0.50 & 0.62 & 0.51 \\
\hline 0.57 & 0.51 & 0.57 & 0.51 & 0.50 & 0.55 & 0.53 & 0.53 & 0.49 & 0.62 & 0.63 & 0.68 & 0.37 & 0.44 & 0.34 \\
\hline 0.55 & 0.48 & 0.55 & 0.48 & 0.48 & 0.55 & 0.52 & 0.51 & 0.48 & 0.64 & 0.63 & 0.69 & 0.37 & 0.42 & 0.30 \\
\hline 0.78 & 0.72 & 0.78 & 0.69 & 0.67 & 0.71 & 0.69 & 0.71 & 0.63 & 0.75 & 0.78 & 0.79 & 0.50 & 0.62 & 0.51 \\
\hline 0.56 & 0.50 & 0.56 & 0.50 & 0.49 & 0.54 & 0.52 & 0.52 & 0.48 & 0.61 & 0.62 & 0.67 & 0.37 & 0.43 & 0.33 \\
\hline 0.53 & 0.46 & 0.53 & 0.47 & 0.46 & 0.54 & 0.50 & 0.50 & 0.47 & 0.63 & 0.62 & 0.68 & 0.36 & 0.40 & 0.30 \\
\hline 0.98 & 0.94 & 0.98 & 0.92 & 0.89 & 0.88 & 0.89 & 0.92 & 0.82 & 0.83 & 0.90 & 0.82 & 0.56 & 0.75 & 0.64 \\
\hline
\end{tabular}


Experiment

LEU-COMP-THERM-053-002 LEU-COMP-THERM-053-003 LEU-COMP-THERM-053-004 LEU-COMP-THERM-053-005 LEU-COMP-THERM-053-006 LEU-COMP-THERM-053-007 LEU-COMP-THERM-053-008 LEU-COMP-THERM-053-009 LEU-COMP-THERM-053-010 LEU-COMP-THERM-053-011 LEU-COMP-THERM-053-012 LEU-COMP-THERM-053-013 LEU-COMP-THERM-053-014 LEU-COMP-THERM-054-001 LEU-COMP-THERM-054-002 LEU-COMP-THERM-054-003 LEU-COMP-THERM-054-004 LEU-COMP-THERM-054-005 LEU-COMP-THERM-054-006 LEU-COMP-THERM-054-007 LEU-COMP-THERM-054-008 LEU-COMP-THERM-055-001 LEU-COMP-THERM-055-002 LEU-COMP-THERM-057-001 LEU-COMP-THERM-057-002 LEU-COMP-THERM-057-003 LEU-COMP-THERM-057-004 LEU-COMP-THERM-057-005 LEU-COMP-THERM-057-006 LEU-COMP-THERM-057-007 LEU-COMP-THERM-057-008 LEU-COMP-THERM-057-009 LEU-COMP-THERM-057-010 LEU-COMP-THERM-057-011 LEU-COMP-THERM-057-012 LEU-COMP-THERM-057-013 LEU-COMP-THERM-057-014 LEU-COMP-THERM-057-015 LEU-COMP-THERM-057-016 LEU-COMP-THERM-057-017 LEU-COMP-THERM-057-018 LEU-COMP-THERM-057-019_

\begin{tabular}{|c|c|c|c|c|c|c|c|c|c|c|c|c|c|c|}
\hline 1 & 2 & 3 & 4 & 5 & 6 & 7 & 8 & 9 & 10 & 11 & 12 & 13 & 14 & 15 \\
\hline 0.98 & 0.94 & 0.98 & 0.91 & 0.89 & 0.88 & 0.89 & 0.92 & 0.81 & 0.83 & 0.90 & 0.82 & 0.56 & 0.75 & 0.64 \\
\hline 0.99 & 0.94 & 0.98 & 0.92 & 0.89 & 0.88 & 0.89 & 0.91 & 0.81 & 0.82 & 0.90 & 0.81 & 0.55 & 0.74 & .6 \\
\hline 0.99 & 0.95 & 0.98 & 0.92 & 0.90 & 0.88 & 0.90 & 0.92 & 0.82 & 0.82 & 0.90 & 0.81 & 0.54 & 0.73 & \\
\hline 0.99 & 0.96 & 0.98 & 0.93 & 0.91 & 0.88 & 0.90 & 0.92 & 0.82 & 0.81 & 0.89 & 0.80 & 0.52 & 0.72 & \\
\hline 0.99 & 0.96 & 0.98 & 0.93 & 0.91 & 0.88 & 0.90 & 0.92 & 0.82 & 0.81 & 0.89 & 0.80 & 0.52 & 0.72 & \\
\hline 0.99 & 0.96 & 0.98 & 0.93 & 0.91 & 0.88 & 0.90 & 0.92 & 0.82 & 0.81 & 0.89 & 0.80 & 0.52 & 0.72 & \\
\hline 0.99 & 0.96 & 0.98 & 0.93 & 0.91 & 0.88 & 0.90 & 0.92 & 0.82 & 0.81 & 0.89 & 0.80 & 0.52 & 0.72 & \\
\hline 0.99 & 0.96 & 0.98 & 0.93 & 0.91 & 0.88 & 0.90 & 0.92 & 0.83 & 0.81 & 0.90 & 0.80 & 0.52 & 0.72 & \\
\hline 0.99 & 0.96 & 0.98 & 0.93 & 0.91 & 0.88 & 0.90 & 0.92 & 0.82 & 0.81 & 0.90 & 0.80 & 0.52 & 0.72 & \\
\hline 0.99 & 0.96 & 0.98 & 0.93 & 0.91 & 0.88 & 0.90 & 0.92 & 0.83 & 0.81 & 0.90 & 0.80 & 0.52 & 0.72 & \\
\hline 0.99 & 0.96 & 0.98 & 0.93 & 0.91 & 0.89 & 0.90 & 0.92 & 0.83 & 0.82 & 0.90 & 0.81 & 0.53 & 0.72 & \\
\hline 0.99 & 0.96 & 0.98 & 0.94 & 0.92 & 0.88 & 0.90 & 0.92 & 0.83 & 0.81 & 0.90 & 0.80 & 0.52 & 0.72 & \\
\hline 0.99 & 0.96 & 0.98 & 0.93 & 0.91 & 0.88 & 0.90 & 0.92 & 0.83 & 0.81 & 0.90 & 0.80 & 0.52 & 0.72 & \\
\hline 0.87 & 0.82 & 0.86 & 0.90 & 0.88 & 0.98 & 0.96 & 0.96 & 0.94 & 0.94 & 96 & 0.92 & 0.63 & 0.73 & \\
\hline 0.87 & 0.82 & 0.86 & 0.90 & 0.88 & 0.98 & 0.96 & 0.97 & 0.95 & 0.93 & 0.96 & 0.92 & 0.63 & 0.73 & \\
\hline 0.87 & 0.82 & 0.87 & 0.90 & 0.89 & 0.98 & 0.96 & 0.97 & 0.95 & 0.93 & 0.96 & 0.92 & 0.62 & 0.73 & \\
\hline 0.87 & 0.82 & 0.87 & 0.91 & 0.89 & 0.98 & 0.96 & 0.97 & 0.95 & 0.93 & 0.96 & 0.92 & 0.62 & 0.73 & \\
\hline 0.87 & 0.82 & 0.87 & 0.90 & 0.89 & 0.98 & 0.96 & 0.97 & 0.95 & 0.93 & 0.96 & 0.92 & 0.63 & 0.73 & \\
\hline 0.87 & 0.82 & 0.86 & 0.90 & 0.88 & 0.98 & 0.96 & 0.97 & 0.95 & 0.93 & 0.96 & 0.92 & 0.63 & 0.73 & \\
\hline 0.87 & 0.82 & 0.86 & 0.90 & 0.88 & 0.98 & 0.96 & 0.96 & 0.94 & 0.94 & 0.96 & 0.92 & 0.63 & 0.73 & \\
\hline 0.87 & 0.82 & 0.87 & 0.90 & 0.88 & 0.98 & 0.96 & 0.97 & 0.95 & 0.93 & 0.96 & 0.92 & 0.63 & 0.73 & \\
\hline 0.72 & 0.53 & 0.71 & 0.57 & 0.52 & 0.77 & 0.67 & 0.71 & 0.60 & 0.87 & 0.81 & 0.84 & 0.84 & 0.79 & \\
\hline 0.69 & 0.50 & 0.68 & 0.55 & 0.50 & 0.76 & 0.66 & 0.69 & 0.59 & 0.86 & 0.79 & 0.83 & 0.85 & 0.78 & \\
\hline 0.98 & 0.96 & 0.98 & 0.94 & 0.93 & 0.90 & 0.92 & 0.92 & 0.85 & 0.83 & 0.91 & 0.81 & 0.51 & 0.70 & \\
\hline 0.98 & 0.95 & 0.97 & 0.94 & 0.93 & 0.90 & 0.92 & 0.92 & 0.85 & 0.83 & 0.91 & 0.82 & 0.51 & 0.70 & \\
\hline 0.98 & 0.94 & 0.97 & 0.93 & 0.92 & 0.90 & 0.91 & 0.92 & 0.85 & 0.84 & 0.91 & 0.82 & 0.52 & 0.70 & \\
\hline 0.98 & 0.94 & 0.97 & 0.93 & 0.92 & 0.90 & 0.91 & 0.92 & 0.85 & 0.84 & 0.91 & 0.82 & 0.52 & 0.70 & \\
\hline 0.98 & 0.95 & 0.97 & 0.94 & 0.92 & 0.91 & 0.92 & 0.92 & 0.85 & 0.84 & 0.91 & 0.82 & 0.51 & 0.70 & \\
\hline 0.98 & 0.95 & 0.97 & 0.94 & 0.92 & 0.91 & 0.92 & 0.92 & 0.85 & 0.84 & 0.91 & 0.82 & 0.51 & 0.70 & \\
\hline 0.98 & 0.96 & 0.97 & 0.94 & 0.93 & 0.91 & 0.92 & 0.93 & 0.86 & 0.83 & 0.91 & 0.82 & 0.51 & 0.69 & \\
\hline 0.98 & 0.96 & 0.98 & 0.95 & 0.94 & 0.91 & 0.93 & 0.93 & 0.87 & 0.83 & 0.91 & 0.82 & 0.50 & 0.69 & \\
\hline 0.98 & 0.98 & 0.98 & 0.96 & 0.95 & 0.90 & 0.93 & 0.94 & 0.87 & 0.81 & 0.90 & 0.80 & 0.48 & 0.69 & \\
\hline 0.98 & 0.98 & 0.98 & 0.96 & 0.95 & 0.90 & 0.93 & 0.94 & 0.87 & 0.81 & 0.90 & 0.80 & 0.48 & 0.69 & \\
\hline 0.99 & 0.98 & 0.98 & 0.97 & 0.95 & 0.90 & 0.93 & 0.94 & 0.87 & 0.81 & 0.90 & 0.80 & 0.48 & 0.69 & \\
\hline 0.99 & 0.98 & 0.98 & 0.97 & 0.95 & 0.90 & 0.93 & 0.94 & 0.87 & 0.80 & 0.90 & 0.79 & 0.48 & 0.69 & \\
\hline 0.99 & 0.98 & 0.98 & 0.9 & 0.95 & 0.89 & 0.93 & 0.94 & 0.87 & 0.79 & 0.89 & 0.79 & 0.47 & 0.69 & \\
\hline 0.99 & 0.98 & 0.98 & 0.97 & 0.95 & 0.90 & 0.93 & 0.94 & 0.87 & 0.80 & 0.90 & 0.79 & 0.48 & 0.69 & \\
\hline 0.99 & 0.98 & 0.98 & 0.96 & 0.95 & 0.89 & 0.93 & 0.94 & 0.86 & 0.80 & 0.89 & 0.79 & 0.48 & 0.70 & \\
\hline 0.98 & 0.96 & 0.98 & 0.94 & 0.93 & 0.90 & 0.92 & 0.92 & 0.85 & 0.82 & 0.91 & 0.81 & 0.50 & 0.69 & \\
\hline 0.99 & 0.98 & 0.98 & 0.96 & 0.95 & 0.90 & 0.93 & 0.94 & 0.87 & 0.81 & 0.90 & 0.80 & 0.49 & 0.70 & \\
\hline 0.99 & 0.98 & 0.98 & 0.96 & 0.95 & 0.90 & 0.93 & 0.94 & 0.87 & 0.81 & 0.91 & 0.81 & 0.49 & 0.70 & \\
\hline 0.99 & 0.98 & 0.98 & 0.96 & 0.95 & 0.90 & 0.93 & 0.94 & 0.87 & 0.80 & 0.90 & 0.80 & 0.49 & 0.70 & \\
\hline
\end{tabular}


Experiment

LEU-COMP-THERM-057-020 LEU-COMP-THERM-057-021 LEU-COMP-THERM-057-022 LEU-COMP-THERM-057-023 LEU-COMP-THERM-057-024 LEU-COMP-THERM-057-025 LEU-COMP-THERM-057-026 LEU-COMP-THERM-057-027 LEU-COMP-THERM-057-028 LEU-COMP-THERM-057-029 LEU-COMP-THERM-057-030 LEU-COMP-THERM-057-031 LEU-COMP-THERM-057-032 LEU-COMP-THERM-057-033 LEU-COMP-THERM-057-034 LEU-COMP-THERM-057-035 LEU-COMP-THERM-057-036 LEU-COMP-THERM-058-001 LEU-COMP-THERM-058-002 LEU-COMP-THERM-058-003 LEU-COMP-THERM-058-004 LEU-COMP-THERM-058-005 LEU-COMP-THERM-058-006 LEU-COMP-THERM-058-007 LEU-COMP-THERM-058-008 LEU-COMP-THERM-058-009 LEU-COMP-THERM-061-001 LEU-COMP-THERM-061-002 LEU-COMP-THERM-061-003 LEU-COMP-THERM-061-004 LEU-COMP-THERM-061-005 LEU-COMP-THERM-061-006 LEU-COMP-THERM-061-007 LEU-COMP-THERM-061-008 LEU-COMP-THERM-061-009 LEU-COMP-THERM-061-010 LEU-COMP-THERM-062-001 LEU-COMP-THERM-062-002 LEU-COMP-THERM-062-003 LEU-COMP-THERM-062-004 LEU-COMP-THERM-062-005 LEU-COMP-THERM-062-006

\begin{tabular}{|c|c|c|c|c|c|c|c|c|c|c|c|c|c|c|}
\hline 1 & 2 & 3 & 4 & 5 & 6 & 7 & 8 & 9 & 10 & 11 & 12 & 13 & 14 & 15 \\
\hline 0.99 & 0.98 & 0.98 & 0.96 & 0.95 & 0.90 & 0.93 & 0.94 & 0.87 & 0.81 & 0.90 & 0.80 & 0.49 & 0.70 & 0 \\
\hline 0.99 & 0.98 & 0.98 & 0.96 & 0.95 & 0.90 & 0.93 & 0.94 & 0.87 & 0.81 & 0.90 & 0.80 & 0.49 & 0.70 & \\
\hline 0.99 & 0.98 & 0.98 & 0.96 & 0.95 & 0.90 & 0.93 & 0.94 & 0.87 & 0.80 & 0.90 & 0.80 & 0.48 & 0.70 & \\
\hline 0.99 & 0.98 & 0.98 & 0.96 & 0.95 & 0.90 & 0.93 & 0.94 & 0.87 & 0.81 & 0.90 & 0.80 & 0.49 & 0.70 & \\
\hline 0.98 & 0.96 & 0.97 & 0.94 & 0.93 & 0.91 & 0.92 & 0.93 & 0.86 & 0.83 & 0.91 & 0.82 & 0.51 & 0.70 & \\
\hline 0.99 & 0.98 & 0.98 & 0.96 & 0.95 & 0.90 & 0.93 & 0.94 & 0.87 & 0.81 & 0.90 & 0.80 & 0.50 & 0.70 & \\
\hline 0.99 & 0.96 & 0.98 & 0.95 & 0.94 & 0.91 & 0.93 & 0.93 & 0.86 & 0.83 & 0.91 & 0.82 & 0.51 & 0.70 & \\
\hline 0.99 & 0.97 & 0.98 & 0.96 & 0.94 & 0.91 & 0.93 & 0.93 & 0.87 & 0.82 & 0.91 & 0.81 & 0.50 & 0.70 & \\
\hline 0.99 & 0.98 & 0.98 & 0.96 & 0.95 & 0.90 & 0.93 & 0.94 & 0.87 & 0.81 & 0.90 & 0.80 & 0.49 & 0.70 & \\
\hline 0.99 & 0.98 & 0.98 & 0.96 & 0.95 & 0.90 & 0.93 & 0.94 & 0.87 & 0.81 & 0.90 & 0.80 & 0.49 & 0.70 & \\
\hline 0.98 & 0.95 & 0.97 & 0.94 & 0.92 & 0.90 & 0.92 & 0.92 & 0.85 & 0.83 & 0.91 & 0.82 & 0.51 & 0.70 & \\
\hline 0.99 & 0.98 & 0.98 & 0.96 & 0.95 & 0.90 & 0.93 & 0.94 & 0.87 & 0.82 & 0.91 & 0.81 & 0.50 & 0.70 & \\
\hline 0.98 & 0.94 & 0.97 & 0.93 & 0.92 & 0.91 & 0.91 & 0.92 & 0.85 & 0.84 & 0.91 & 0.83 & 0.52 & 0.70 & \\
\hline 0.98 & 0.95 & 0.97 & 0.94 & 0.93 & 0.91 & 0.92 & 0.92 & 0.85 & 0.84 & 91 & 0.82 & 0.52 & 0.70 & \\
\hline 0.98 & 0.95 & 0.97 & 0.93 & 0.92 & 0.91 & 0.92 & 0.92 & 0.85 & 0.84 & 0.91 & 0.83 & 0.52 & 0.70 & \\
\hline 0.98 & 0.95 & 0.97 & 0.93 & 0.92 & 0.91 & 0.92 & 0.92 & 0.85 & 0.84 & 0.91 & 0.83 & 0.52 & 0.70 & \\
\hline 0.98 & 0.97 & 0.98 & 0.95 & 0.94 & 0.91 & 0.93 & 0.93 & 0.86 & 0.82 & 0.91 & 0.81 & 0.50 & 0.70 & \\
\hline 0.87 & 0.82 & 0.87 & 0.90 & 0.88 & 0.98 & 0.96 & 0.97 & 0.95 & 0.93 & 0.96 & 0.92 & 0.63 & 0.73 & \\
\hline 0.87 & 0.82 & 0.87 & 0.90 & 0.88 & 0.98 & 0.96 & 0.97 & 0.95 & 0.93 & 0.96 & 0.92 & 0.63 & 0.73 & \\
\hline 0.87 & 0.82 & 0.86 & 0.90 & 0.88 & 0.98 & 0.96 & 0.97 & 0.95 & 0.93 & 0.96 & 0.92 & 0.63 & 0.73 & \\
\hline 0.87 & 0.82 & 0.86 & 0.90 & 0.88 & 0.98 & 0.96 & 0.97 & 0.95 & 0.94 & 0.96 & 0.92 & 0.63 & 0.73 & \\
\hline 0.87 & 0.82 & 0.87 & 0.90 & 0.88 & 0.98 & 0.96 & 0.97 & 0.95 & 0.93 & 0.96 & 0.92 & 0.63 & 0.73 & \\
\hline 0.87 & 0.82 & 0.86 & 0.90 & 0.88 & 0.98 & 0.96 & 0.97 & 0.95 & 0.94 & 0.96 & 0.92 & 0.63 & 0.73 & \\
\hline 0.88 & 0.84 & 0.87 & 0.92 & 0.90 & 0.98 & 0.97 & 0.97 & 0.95 & 0.92 & 0.95 & 0.91 & 0.61 & 0.72 & \\
\hline 0.88 & 0.83 & 0.87 & 0.91 & 0.90 & 0.98 & 0.96 & 0.97 & 0.95 & 0.92 & 0.96 & 0.91 & 0.61 & 0.72 & \\
\hline 0.88 & 0.83 & 0.87 & 0.91 & 0.90 & 0.98 & 0.96 & 0.97 & 0.95 & 0.92 & 0.96 & 0.91 & 0.61 & 0.72 & \\
\hline 0.99 & 0.94 & 0.98 & 0.92 & 0.89 & 0.90 & 0.90 & 0.91 & 0.82 & 0.85 & 0.92 & 0.83 & 0.57 & 0.74 & \\
\hline 0.99 & 0.93 & 0.98 & 0.91 & 0.89 & 0.90 & 0.90 & 0.91 & 0.82 & 0.85 & 0.92 & 0.84 & 0.57 & 0.74 & \\
\hline 0.99 & 0.94 & 0.98 & 0.91 & 0.89 & 0.89 & 0.90 & 0.91 & 0.82 & 0.85 & 0.91 & 0.83 & 0.57 & 0.74 & \\
\hline 0.99 & 0.94 & 0.98 & 0.91 & 0.89 & 0.89 & 0.90 & 0.91 & 0.82 & 0.85 & 0.91 & 0.83 & 0.57 & 0.74 & \\
\hline 0.99 & 0.94 & 0.98 & 0.92 & 0.90 & 0.89 & 0.90 & 0.91 & 0.82 & 0.84 & 0.91 & 0.83 & 0.56 & 0.74 & \\
\hline 0.99 & 0.94 & 0.98 & 0.92 & 0.89 & 0.89 & 0.90 & 0.91 & 0.82 & 0.84 & 0.91 & 0.83 & 0.56 & 0.74 & \\
\hline 0.99 & 0.95 & 0.98 & 0.92 & 0.90 & 0.89 & 0.90 & 0.92 & 0.82 & 0.84 & 0.91 & 0.83 & 0.55 & 0.74 & \\
\hline 0.99 & 0.94 & 0.98 & 0.92 & 0.90 & 0.89 & 0.90 & 0.92 & 0.82 & 0.84 & 0.91 & 0.83 & 0.56 & 0.74 & \\
\hline 0.99 & 0.95 & 0.98 & 0.93 & 0.91 & 0.90 & 0.91 & 0.92 & 0.84 & 0.85 & 0.92 & 0.84 & 0.55 & 0.73 & \\
\hline 0.95 & 0.85 & 0.93 & 0.8 & 0.82 & 0.8 & 0.86 & 0.87 & 0.78 & 0.88 & 0.92 & 0.86 & 0.60 & 0.72 & \\
\hline 0.98 & 0.91 & 0.96 & 0.89 & 0.86 & 0.89 & 0.88 & 0.89 & 0.79 & 0.85 & 0.90 & 0.83 & 0.57 & 0.73 & \\
\hline 0.98 & 0.91 & 0.96 & 0.90 & 0.86 & 0.90 & 0.88 & 0.90 & 0.80 & 0.86 & 0.91 & 0.84 & 0.58 & 0.73 & \\
\hline 0.98 & 0.90 & 0.96 & 0.89 & 0.86 & 0.90 & 0.88 & 0.90 & 0.80 & 0.86 & 0.91 & 0.84 & 0.58 & 0.73 & \\
\hline 0.98 & 0.91 & 0.96 & 0.89 & 0.86 & 0.90 & 0.88 & 0.90 & 0.80 & 0.86 & 0.91 & 0.83 & 0.58 & 0.73 & \\
\hline 0.98 & 0.91 & 0.96 & 0.90 & 0.86 & 0.89 & 0.88 & 0.90 & 0.80 & 0.85 & 0.91 & 0.83 & 0.57 & 0.73 & \\
\hline 0.98 & 0.90 & 0.96 & 0.89 & 0.86 & 0.89 & 0.88 & 0.89 & 0.79 & 0.85 & 0.91 & 0.83 & 0.58 & 0.73 & \\
\hline
\end{tabular}


Experiment

LEU-COMP-THERM-062-007 LEU-COMP-THERM-062-008 LEU-COMP-THERM-062-009 LEU-COMP-THERM-062-010 LEU-COMP-THERM-062-011 LEU-COMP-THERM-062-012 LEU-COMP-THERM-062-013 LEU-COMP-THERM-062-014 LEU-COMP-THERM-062-015 LEU-COMP-THERM-065-001 LEU-COMP-THERM-065-002 LEU-COMP-THERM-065-003 LEU-COMP-THERM-065-004 LEU-COMP-THERM-065-005 LEU-COMP-THERM-065-006 LEU-COMP-THERM-065-007 LEU-COMP-THERM-065-008 LEU-COMP-THERM-065-009 LEU-COMP-THERM-065-010 LEU-COMP-THERM-065-011 LEU-COMP-THERM-065-012 LEU-COMP-THERM-065-013 LEU-COMP-THERM-065-014 LEU-COMP-THERM-065-015 LEU-COMP-THERM-065-016 LEU-COMP-THERM-065-017 LEU-COMP-THERM-066-004 LEU-COMP-THERM-066-005 LEU-COMP-THERM-066-006 LEU-COMP-THERM-066-007 LEU-COMP-THERM-066-008 LEU-COMP-THERM-066-009 LEU-COMP-THERM-066-010 LEU-COMP-THERM-068-004 LEU-COMP-THERM-068-005 LEU-COMP-THERM-068-006 LEU-COMP-THERM-068-007 LEU-COMP-THERM-068-008 LEU-COMP-THERM-068-009 LEU-COMP-THERM-068-010 LEU-COMP-THERM-068-011 LEU-COMP-THERM-068-012

\begin{tabular}{|c|c|c|c|c|c|c|c|c|c|c|c|c|c|c|}
\hline 1 & 2 & 3 & 4 & 5 & 6 & 7 & 8 & 9 & 10 & 11 & 12 & 13 & 14 & 15 \\
\hline 0.97 & 0.90 & 0.96 & 0.88 & 0.85 & 0.89 & 0.87 & 0.89 & 0.78 & 0.86 & 0.91 & 0.83 & 0.58 & 0.73 & 0.59 \\
\hline 0.98 & 0.90 & 0.96 & 0.89 & 0.86 & 0.89 & 0.88 & 0.89 & 0.79 & 0.85 & 0.91 & 0.83 & 0.58 & 0.73 & 0.59 \\
\hline 0.98 & 0.91 & 0.96 & 0.89 & 0.86 & 0.89 & 0.88 & 0.90 & 0.79 & 0.85 & 0.91 & 0.83 & 0.57 & 0.73 & 0.59 \\
\hline 0.98 & 0.90 & 0.96 & 0.89 & 0.86 & 0.89 & 0.88 & 0.89 & 0.79 & 0.85 & 0.91 & 0.83 & 0.58 & 0.73 & 0.59 \\
\hline 0.98 & 0.91 & 0.96 & 0.89 & 0.86 & 0.89 & 0.88 & 0.90 & 0.79 & 0.85 & 0.91 & 0.83 & 0.57 & 0.73 & 0.59 \\
\hline 0.98 & 0.90 & 0.96 & 0.89 & 0.86 & 0.89 & 0.88 & 0.89 & 0.79 & 0.85 & 0.91 & 0.83 & 0.58 & 0.73 & 0.59 \\
\hline 0.97 & 0.90 & 0.96 & 0.89 & 0.85 & 0.89 & 0.87 & 0.89 & 0.79 & 0.86 & 0.91 & 0.83 & 0.58 & 0.73 & 0.59 \\
\hline 0.98 & 0.91 & 0.96 & 0.89 & 0.86 & 0.89 & 0.88 & 0.90 & 0.79 & 0.85 & 0.91 & 0.83 & 0.57 & 0.73 & 0.59 \\
\hline 0.98 & 0.90 & 0.96 & 0.89 & 0.86 & 0.89 & 0.88 & 0.89 & 0.79 & 0.85 & 0.91 & 0.83 & 0.57 & 0.73 & 0.59 \\
\hline 0.97 & 0.89 & 0.95 & 0.87 & 0.84 & 0.89 & 0.87 & 0.88 & 0.78 & 0.86 & 0.91 & 0.84 & 0.59 & 0.73 & 0.58 \\
\hline 0.96 & 0.87 & 0.95 & 0.88 & 0.84 & 0.91 & 0.88 & 0.90 & 0.80 & 0.89 & 0.93 & 0.86 & 0.62 & 0.75 & 0.59 \\
\hline 0.97 & 0.88 & 0.95 & 0.86 & 0.83 & 0.89 & 0.86 & 0.88 & 0.77 & 0.87 & 0.91 & 0.84 & 0.61 & 0.74 & 0.58 \\
\hline 0.96 & 0.87 & 0.95 & 0.86 & 0.82 & 0.89 & 0.86 & 0.88 & 0.77 & 0.87 & 0.91 & 0.84 & 0.61 & 0.74 & 0.58 \\
\hline 0.97 & 0.88 & 0.95 & 0.87 & 0.84 & 0.89 & 0.87 & 0.88 & 0.78 & 0.86 & 0.91 & 0.84 & 0.59 & 0.73 & 0.58 \\
\hline 0.97 & 0.89 & 0.95 & 0.88 & 0.85 & 0.91 & 0.88 & 0.90 & 0.80 & 0.88 & 0.92 & 0.85 & 0.60 & 0.74 & 0.59 \\
\hline 0.97 & 0.88 & 0.95 & 0.88 & 0.84 & 0.90 & 0.88 & 0.89 & 0.79 & 0.88 & 0.92 & 0.85 & 0.61 & 0.74 & 0.59 \\
\hline 0.97 & 0.88 & 0.95 & 0.88 & 0.84 & 0.91 & 0.88 & 0.90 & 0.80 & 0.89 & 0.92 & 0.86 & 0.61 & 0.75 & 0.59 \\
\hline 0.97 & 0.88 & 0.95 & 0.88 & 0.84 & 0.90 & 0.88 & 0.90 & 0.79 & 0.88 & 0.92 & 0.85 & 0.61 & 0.74 & 0.59 \\
\hline 0.97 & 0.89 & 0.95 & 0.87 & 0.84 & 0.89 & 0.87 & 0.89 & 0.78 & 0.86 & 0.91 & 0.84 & 0.59 & 0.74 & 0.59 \\
\hline 0.97 & 0.88 & 0.95 & 0.87 & 0.83 & 0.89 & 0.86 & 0.88 & 0.77 & 0.87 & 0.91 & 0.84 & 0.60 & 0.74 & 0.58 \\
\hline 0.97 & 0.88 & 0.95 & 0.87 & 0.84 & 0.89 & 0.87 & 0.89 & 0.78 & 0.87 & 0.91 & 0.84 & 0.60 & 0.74 & 0.58 \\
\hline 0.97 & 0.89 & 0.95 & 0.87 & 0.84 & 0.89 & 0.87 & 0.89 & 0.78 & 0.86 & 0.91 & 0.84 & 0.59 & 0.74 & 0.59 \\
\hline 0.97 & 0.88 & 0.95 & 0.87 & 0.83 & 0.89 & 0.86 & 0.88 & 0.77 & 0.87 & 0.91 & 0.84 & 0.60 & 0.74 & 0.58 \\
\hline 0.97 & 0.88 & 0.95 & 0.87 & 0.84 & 0.89 & 0.87 & 0.88 & 0.78 & 0.87 & 0.91 & 0.84 & 0.60 & 0.74 & 0.58 \\
\hline 0.97 & 0.88 & 0.95 & 0.87 & 0.84 & 0.89 & 0.87 & 0.89 & 0.78 & 0.87 & 0.91 & 0.84 & 0.60 & 0.74 & 0.59 \\
\hline 0.97 & 0.88 & 0.95 & 0.87 & 0.83 & 0.89 & 0.86 & 0.88 & 0.77 & 0.87 & 0.91 & 0.84 & 0.60 & 0.74 & 0.58 \\
\hline 0.92 & 0.92 & 0.93 & 0.89 & 0.90 & 0.87 & 0.91 & 0.89 & 0.87 & 0.79 & 0.87 & 0.80 & 0.65 & 0.77 & 0.71 \\
\hline 0.94 & 0.96 & 0.95 & 0.93 & 0.94 & 0.86 & 0.92 & 0.91 & 0.89 & 0.76 & 0.86 & 0.77 & 0.55 & 0.73 & 0.69 \\
\hline 0.91 & 0.97 & 0.92 & 0.94 & 0.96 & 0.83 & 0.91 & 0.89 & 0.89 & 0.69 & 0.82 & 0.71 & 0.44 & 0.65 & 0.64 \\
\hline 0.93 & 0.90 & 0.94 & 0.89 & 0.89 & 0.91 & 0.92 & 0.91 & 0.88 & 0.85 & 0.91 & 0.85 & 0.67 & 0.77 & 0.67 \\
\hline 0.95 & 0.95 & 0.96 & 0.94 & 0.94 & 0.91 & 0.94 & 0.93 & 0.90 & 0.82 & 0.91 & 0.83 & 0.58 & 0.74 & 0.65 \\
\hline 0.96 & 0.95 & 0.96 & 0.94 & 0.94 & 0.91 & 0.94 & 0.93 & 0.90 & 0.82 & 0.91 & 0.83 & 0.57 & 0.73 & 0.65 \\
\hline 0.93 & 0.97 & 0.93 & 0.95 & 0.97 & 0.88 & 0.94 & 0.92 & 0.91 & 0.76 & 0.87 & 0.76 & 0.45 & 0.65 & 0.59 \\
\hline 0.03 & 0.03 & 0.03 & 0.01 & 0.01 & 0.00 & 0.00 & 0.00 & 0.00 & 0.01 & 0.01 & 0.01 & 0.00 & 0.00 & 0.00 \\
\hline 0.03 & 0.03 & 0.03 & 0.01 & 0.01 & 0.00 & 0.00 & 0.00 & 0.00 & 0.01 & 0.01 & 0.01 & 0.00 & 0.00 & 0.00 \\
\hline 0.04 & 0.04 & 0.04 & 0.02 & 0.02 & 0.00 & 0.00 & 0.00 & 0.00 & 0.01 & 0.01 & 0.01 & 0.00 & 0.00 & 0.00 \\
\hline 0.04 & 0.04 & 0.04 & 0.02 & 0.02 & 0.00 & 0.00 & 0.00 & 0.00 & 0.01 & 0.02 & 0.02 & 0.00 & 0.00 & 0.00 \\
\hline 0.05 & 0.05 & 0.05 & 0.01 & 0.01 & 0.00 & 0.00 & 0.00 & 0.00 & 0.02 & 0.02 & 0.02 & 0.00 & 0.00 & 0.00 \\
\hline 0.02 & 0.02 & 0.02 & 0.01 & 0.01 & 0.00 & 0.00 & 0.00 & 0.00 & 0.01 & 0.01 & 0.01 & 0.00 & 0.00 & 0.00 \\
\hline 0.02 & 0.03 & 0.03 & 0.01 & 0.01 & 0.00 & 0.00 & 0.00 & 0.00 & 0.01 & 0.01 & 0.01 & 0.00 & 0.00 & 0.00 \\
\hline 0.03 & 0.03 & 0.03 & 0.02 & 0.02 & 0.00 & 0.00 & 0.00 & 0.00 & 0.02 & 0.02 & 0.02 & 0.00 & 0.00 & 0.00 \\
\hline 0.05 & 0.05 & 0.05 & 0.02 & 0.01 & 0.00 & 0.00 & 0.00 & 0.01 & 0.02 & 0.02 & 0.02 & 0.00 & 0.00 & 0.00 \\
\hline
\end{tabular}


1182

1183

1184

1185

1186

1187

1188

1189

1190

1191

1192

1193

1194

1195

1196

1197

1198

1199

1200

1201

1202

1203

1204

1205

1206

1207

1208

1209

1210

1211

1212

1213

1214

1215

1216

1217

1218
Experiment

LEU-COMP-THERM-068-013 LEU-COMP-THERM-068-014 LEU-COMP-THERM-068-015 LEU-COMP-THERM-068-016 LEU-COMP-THERM-068-017

LEU-COMP-THERM-069-001 LEU-COMP-THERM-069-002 LEU-COMP-THERM-069-003 LEU-COMP-THERM-069-004 LEU-COMP-THERM-069-005 LEU-COMP-THERM-070-001 LEU-COMP-THERM-070-002 LEU-COMP-THERM-070-003 LEU-COMP-THERM-070-004 LEU-COMP-THERM-070-005 LEU-COMP-THERM-070-006 LEU-COMP-THERM-070-007 LEU-COMP-THERM-070-008 LEU-COMP-THERM-070-009 LEU-COMP-THERM-070-010 LEU-COMP-THERM-070-011 LEU-COMP-THERM-070-012 LEU-COMP-THERM-071-001 LEU-COMP-THERM-071-002 LEU-COMP-THERM-071-003 LEU-COMP-THERM-071-004 LEU-COMP-THERM-072-001 LEU-COMP-THERM-072-002 LEU-COMP-THERM-072-003 LEU-COMP-THERM-072-004 LEU-COMP-THERM-072-005 LEU-COMP-THERM-072-006 LEU-COMP-THERM-072-007 LEU-COMP-THERM-072-008 LEU-COMP-THERM-072-009 LEU-COMP-THERM-073-001 LEU-COMP-THERM-073-002 LEU-COMP-THERM-073-003 LEU-COMP-THERM-073-004 LEU-COMP-THERM-073-005

LEU-COMP-THERM-073-006 LEU-COMP-THERM-073-007_-

\begin{tabular}{|c|c|c|c|c|c|c|c|c|c|c|c|c|c|c|}
\hline 1 & 2 & 3 & 4 & 5 & 6 & 7 & 8 & 9 & 10 & 11 & 12 & 13 & 14 & 15 \\
\hline 0.02 & 0.02 & 0.02 & 0.01 & 0.01 & 0.00 & 0.00 & 0.00 & 0.00 & 0.01 & 0.01 & 0.01 & 0.00 & 0.00 & 0.00 \\
\hline 0.02 & 0.02 & 0.02 & 0.01 & 0.01 & 0.00 & 0.00 & 0.00 & 0.00 & 0.01 & 0.01 & 0.01 & 0.00 & 0.00 & 0.00 \\
\hline 0.02 & 0.02 & 0.02 & 0.01 & 0.01 & 0.00 & 0.00 & 0.00 & 0.00 & 0.01 & 0.01 & 0.01 & 0.00 & 0.00 & 0.00 \\
\hline 0.04 & 0.04 & 0.04 & 0.02 & 0.02 & 0.00 & 0.00 & 0.00 & 0.00 & 0.01 & 0.01 & 0.01 & 0.00 & 0.00 & 0.00 \\
\hline 0.03 & 0.04 & 0.04 & 0.02 & 0.02 & 0.00 & 0.00 & 0.00 & 0.00 & 0.01 & 0.01 & 0.01 & 0.00 & 0.00 & 0.00 \\
\hline 0.97 & 0.90 & 0.96 & 0.88 & 0.85 & 0.88 & 0.86 & 0.89 & 0.78 & 0.85 & 0.91 & 0.84 & 0.59 & 0.75 & 0.63 \\
\hline 0.90 & 0.77 & 0.89 & 0.75 & 0.70 & 0.80 & 0.76 & 0.80 & 0.66 & 0.83 & 0.85 & 0.82 & 0.72 & 0.81 & 0.67 \\
\hline 0.90 & 0.77 & 0.89 & 0.74 & 0.70 & 0.80 & 0.76 & 0.80 & 0.66 & 0.83 & 0.85 & 0.82 & 0.72 & 0.81 & 0.67 \\
\hline 0.98 & 0.91 & 0.97 & 0.90 & 0.87 & 0.89 & 0.88 & 0.90 & 0.80 & 0.86 & 0.91 & 0.84 & 0.59 & 0.75 & 0.63 \\
\hline 0.94 & 0.85 & 0.94 & 0.82 & 0.79 & 0.85 & 0.82 & 0.85 & 0.73 & 0.84 & 0.88 & 0.83 & 0.67 & 0.79 & 0.66 \\
\hline 0.95 & 0.87 & 0.95 & 0.83 & 0.80 & 0.82 & 0.82 & 0.86 & 0.74 & 0.81 & 0.87 & 0.81 & 0.64 & 0.80 & 0.71 \\
\hline 0.95 & 0.87 & 0.95 & 0.83 & 0.80 & 0.83 & 0.82 & 0.86 & 0.74 & 0.82 & 0.87 & 0.81 & 0.65 & 0.80 & 0.71 \\
\hline 0.95 & 0.88 & 0.95 & 0.84 & 0.81 & 0.83 & 0.82 & 0.86 & 0.74 & 0.81 & 0.87 & 0.81 & 0.64 & 0.80 & 0.71 \\
\hline 0.95 & 0.87 & 0.95 & 0.83 & 0.80 & 0.83 & 0.82 & 0.86 & 0.74 & 0.81 & 0.87 & 0.81 & 0.65 & 0.80 & 0.71 \\
\hline 0.95 & 0.88 & 0.95 & 0.84 & 0.81 & 0.83 & 0.82 & 0.86 & 0.74 & 0.81 & 0.87 & 0.81 & 0.65 & 0.80 & 0.71 \\
\hline 0.95 & 0.88 & 0.95 & 0.83 & 0.81 & 0.82 & 0.82 & 0.86 & 0.74 & 0.81 & 0.87 & 0.80 & 0.64 & 0.80 & 0.71 \\
\hline 0.95 & 0.87 & 0.95 & 0.83 & 0.81 & 0.83 & 0.82 & 0.86 & 0.74 & 0.82 & 0.87 & 0.81 & 0.65 & 0.80 & 0.71 \\
\hline 0.95 & 0.88 & 0.95 & 0.84 & 0.81 & 0.83 & 0.83 & 0.86 & 0.75 & 0.81 & 0.87 & 0.81 & 0.64 & 0.80 & 0.71 \\
\hline 0.95 & 0.87 & 0.95 & 0.83 & 0.80 & 0.83 & 0.82 & 0.86 & 0.74 & 0.82 & 0.87 & 0.82 & 0.66 & 0.80 & 0.71 \\
\hline 0.95 & 0.87 & 0.95 & 0.83 & 0.80 & 0.83 & 0.82 & 0.86 & 0.74 & 0.82 & 0.87 & 0.81 & 0.65 & 0.80 & 0.71 \\
\hline 0.95 & 0.88 & 0.95 & 0.84 & 0.81 & 0.83 & 0.83 & 0.86 & 0.75 & 0.81 & 0.87 & 0.81 & 0.64 & 0.80 & 0.71 \\
\hline 0.95 & 0.88 & 0.95 & 0.84 & 0.81 & 0.83 & 0.83 & 0.86 & 0.75 & 0.82 & 0.87 & 0.81 & 0.65 & 0.80 & 0.71 \\
\hline 0.96 & 0.89 & 0.96 & 0.85 & 0.82 & 0.85 & 0.84 & 0.87 & 0.75 & 0.83 & 0.88 & 0.82 & 0.62 & 0.78 & 0.66 \\
\hline 0.97 & 0.90 & 0.96 & 0.86 & 0.83 & 0.85 & 0.84 & 0.88 & 0.76 & 0.82 & 0.88 & 0.81 & 0.61 & 0.77 & 0.66 \\
\hline 0.97 & 0.89 & 0.96 & 0.86 & 0.83 & 0.85 & 0.84 & 0.88 & 0.76 & 0.83 & 0.89 & 0.82 & 0.61 & 0.77 & 0.66 \\
\hline 0.95 & 0.86 & 0.94 & 0.83 & 0.79 & 0.84 & 0.82 & 0.86 & 0.73 & 0.83 & 0.88 & 0.82 & 0.65 & 0.79 & 0.67 \\
\hline 0.96 & 0.98 & 0.95 & 0.96 & 0.96 & 0.88 & 0.93 & 0.92 & 0.87 & 0.77 & 0.87 & 0.76 & 0.43 & 0.63 & 0.56 \\
\hline 0.96 & 0.97 & 0.95 & 0.96 & 0.96 & 0.88 & 0.92 & 0.91 & 0.87 & 0.77 & 0.87 & 0.76 & 0.43 & 0.63 & 0.55 \\
\hline 0.96 & 0.97 & 0.95 & 0.96 & 0.96 & 0.88 & 0.93 & 0.91 & 0.87 & 0.77 & 0.87 & 0.76 & 0.43 & 0.63 & 0.55 \\
\hline 0.96 & 0.97 & 0.96 & 0.96 & 0.96 & 0.89 & 0.93 & 0.92 & 0.88 & 0.78 & 0.88 & 0.78 & 0.44 & 0.65 & 0.56 \\
\hline 0.96 & 0.97 & 0.95 & 0.96 & 0.96 & 0.89 & 0.93 & 0.92 & 0.87 & 0.78 & 0.88 & 0.78 & 0.44 & 0.65 & 0.56 \\
\hline 0.96 & 0.97 & 0.95 & 0.96 & 0.96 & 0.89 & 0.93 & 0.92 & 0.88 & 0.78 & 0.88 & 0.78 & 0.44 & 0.65 & 0.56 \\
\hline 0.95 & 0.87 & 0.95 & 0.85 & 0.81 & 0.84 & 0.83 & 0.87 & 0.75 & 0.83 & 0.88 & 0.82 & 0.62 & 0.78 & 0.66 \\
\hline 0.95 & 0.87 & 0.95 & 0.84 & 0.81 & 0.84 & 0.83 & 0.86 & 0.74 & 0.83 & 0.88 & 0.82 & 0.62 & 0.78 & 0.66 \\
\hline 0.93 & 0.84 & 0.93 & 0.81 & 0.78 & 0.82 & 0.80 & 0.84 & 0.72 & 0.83 & 0.87 & 0.82 & 0.65 & 0.79 & 0.67 \\
\hline 0.97 & 0.89 & 0.96 & 0.86 & 0.83 & 0.86 & 0.85 & 0.88 & 0.76 & 0.85 & 0.90 & 0.83 & 0.61 & 0.77 & 0.65 \\
\hline 0.97 & 0.89 & 0.96 & 0.86 & 0.83 & 0.87 & 0.85 & 0.88 & 0.77 & 0.85 & 0.90 & 0.84 & 0.61 & 0.77 & 0.65 \\
\hline 0.97 & 0.89 & 0.96 & 0.87 & 0.84 & 0.87 & 0.85 & 0.88 & 0.77 & 0.85 & 0.90 & 0.84 & 0.60 & 0.76 & 0.64 \\
\hline 0.97 & 0.91 & 0.97 & 0.88 & 0.85 & 0.86 & 0.85 & 0.89 & 0.77 & 0.82 & 0.89 & 0.81 & 0.59 & 0.76 & 0.66 \\
\hline 0.98 & 0.92 & 0.97 & 0.89 & 0.86 & 0.86 & 0.86 & 0.89 & 0.78 & 0.82 & 0.89 & 0.81 & 0.57 & 0.75 & 0.65 \\
\hline 0.98 & 0.91 & 0.97 & 0.88 & 0.85 & 0.86 & 0.86 & 0.89 & 0.78 & 0.84 & 0.90 & 0.82 & 0.59 & 0.76 & 0.65 \\
\hline 0.98 & 0.90 & 0.97 & 0.88 & 0.84 & 0.86 & 0.86 & 0.89 & 0.77 & 0.84 & 0.90 & 0.83 & 0.59 & 0.76 & 0.65 \\
\hline
\end{tabular}


Experiment

LEU-COMP-THERM-073-008 LEU-COMP-THERM-073-009 LEU-COMP-THERM-073-010 LEU-COMP-THERM-073-011 LEU-COMP-THERM-073-012 LEU-COMP-THERM-073-013 LEU-COMP-THERM-073-014 LEU-COMP-THERM-074-001 LEU-COMP-THERM-074-002 LEU-COMP-THERM-074-003 LEU-COMP-THERM-074-004 LEU-COMP-THERM-075-001 LEU-COMP-THERM-075-002 LEU-COMP-THERM-075-003 LEU-COMP-THERM-075-004 LEU-COMP-THERM-075-005 LEU-COMP-THERM-075-006 LEU-COMP-THERM-076-001 LEU-COMP-THERM-076-002 LEU-COMP-THERM-076-003 LEU-COMP-THERM-076-004 LEU-COMP-THERM-076-005 LEU-COMP-THERM-076-006 LEU-COMP-THERM-076-007 LEU-COMP-THERM-077-001 LEU-COMP-THERM-077-002 LEU-COMP-THERM-077-003 LEU-COMP-THERM-077-004 LEU-COMP-THERM-077-005 LEU-COMP-THERM-078-001 LEU-COMP-THERM-078-002 LEU-COMP-THERM-078-003 LEU-COMP-THERM-078-004 LEU-COMP-THERM-078-005 LEU-COMP-THERM-078-006 LEU-COMP-THERM-078-007 LEU-COMP-THERM-078-008 LEU-COMP-THERM-078-009 LEU-COMP-THERM-078-010 LEU-COMP-THERM-078-011_ LEU-COMP-THERM-078-012 LEU-COMP-THERM-078-013

\begin{tabular}{|c|c|c|c|c|c|c|c|c|c|c|c|c|c|c|}
\hline 1 & 2 & 3 & 4 & 5 & 6 & 7 & 8 & 9 & 10 & 11 & 12 & 13 & 14 & 15 \\
\hline 0.98 & 0.91 & 0.97 & 0.88 & 0.85 & 0.87 & 0.86 & 0.89 & 0.78 & 0.84 & 0.90 & 0.82 & 0.59 & 0.76 & 064 \\
\hline 0.98 & 0.93 & 0.98 & 0.90 & 0.88 & 0.87 & 0.87 & 0.90 & 0.80 & 0.82 & 0.90 & 0.81 & 0.55 & 0.74 &. \\
\hline 0.98 & 0.93 & 0.98 & 0.90 & 0.87 & 0.87 & 0.87 & 0.90 & 0.80 & 0.83 & 0.90 & 0.82 & 0.56 & 0.74 & \\
\hline 0.99 & 0.93 & 0.98 & 0.90 & .88 & 0.87 & 0.88 & 0.90 & 0.80 & 0.83 & 0.90 & 0.82 & 0.55 & 0.74 & \\
\hline 0.97 & 0.88 & 0.96 & 0.86 & 0.83 & 0.87 & 0.85 & 0.88 & 0.77 & 0.86 & 0.91 & 0.85 & 0.62 & 0.76 & \\
\hline 0.97 & 0.88 & 0.96 & 0.86 & 0.83 & 0.87 & 0.85 & 0.88 & 0.77 & 0.86 & 0.91 & 0.85 & 0.62 & 0.76 & \\
\hline 0.97 & 0.88 & 0.96 & 0.86 & 0.83 & 0.87 & 0.85 & 0.88 & 0.77 & 0.87 & 0.91 & 0.85 & 0.62 & 0.76 & \\
\hline 0.95 & 0.94 & 0.95 & 0.93 & 0.92 & 0.89 & 0.91 & 0.90 & 0.85 & 0.80 & 0.89 & 0.79 & 0.47 & 0.65 & \\
\hline 0.95 & 0.93 & 0.95 & 0.92 & 0.91 & 0.89 & 0.91 & 0.90 & 0.85 & 0.82 & 0.89 & 0.81 & 0.49 & 0.66 & \\
\hline 0.95 & 0.97 & 0.95 & 0.95 & 0.95 & 0.88 & 0.92 & 0.91 & 0.87 & 0.77 & 0.87 & 0.76 & 0.43 & 0.63 & \\
\hline 0.95 & 0.96 & 0.94 & 0.95 & 0.95 & 0.88 & 0.92 & 0.91 & 0.87 & 0.77 & 0.87 & 0.76 & 0.43 & 0.63 & \\
\hline 0.94 & 0.86 & 0.94 & 0.82 & 0.79 & 0.82 & 0.81 & 0.85 & 0.73 & 0.82 & 0.87 & 0.81 & 0.67 & 0.81 & \\
\hline 0.94 & 0.85 & 0.94 & 0.81 & 0.78 & 0.82 & 0.81 & 0.84 & 0.73 & 0.83 & 0.87 & 0.82 & 0.68 & 0.81 & \\
\hline 0.94 & 0.85 & 0.94 & 0.81 & 0.78 & 0.83 & 0.81 & 0.85 & 0.73 & 0.83 & 0.88 & 0.83 & 0.69 & 0.82 & \\
\hline 0.95 & 0.88 & 0.95 & 0.84 & 0.81 & 0.82 & 0.82 & 0.86 & 0.74 & 0.81 & 0.87 & 0.80 & 0.64 & 0.80 & \\
\hline 0.95 & 0.88 & 0.95 & 0.84 & 0.81 & 0.82 & 0.82 & 0.86 & 0.74 & 0.81 & 0.87 & 0.80 & 0.64 & 0.80 & \\
\hline 0.95 & 0.88 & 0.95 & 0.84 & 0.81 & 0.83 & 0.83 & 0.86 & 0.75 & 0.81 & 0.87 & 0.81 & 0.64 & 0.80 & \\
\hline 0.61 & 0.40 & 0.60 & 0.46 & 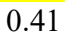 & 0.7 & 0.58 & 0.61 & 0.52 & 0.83 & 0.74 & 0.80 & 0.87 & 0.75 & \\
\hline 0.61 & 0.40 & 0.60 & 0.46 & 0.42 & 0.72 & 0.59 & 0.62 & 0.53 & 0.84 & 0.75 & 0.81 & 0.86 & 0.75 & \\
\hline 0.59 & 0.38 & 0.58 & 0.44 & 0.39 & 0.70 & 0.58 & 0.60 & 0.52 & 0.83 & 0.73 & 0.80 & 0.87 & 0.74 & \\
\hline 0.55 & 0.34 & 0.54 & 0.40 & 0.36 & 0.67 & 0.54 & 0.57 & 0.49 & 0.81 & 0.70 & 0.78 & 0.87 & 0.73 & \\
\hline 0.66 & 0.46 & 0.65 & 0.52 & 0.47 & 0.75 & 0.64 & 0.67 & 0.58 & 0.86 & 0.78 & 0.83 & 0.87 & 0.78 & \\
\hline 0.63 & 0.43 & 0.62 & 0.49 & 0.44 & 0.73 & 0.61 & 0.64 & 0.55 & 0.84 & 0.76 & 0.82 & 0.86 & 0.76 & \\
\hline 0.73 & 0.54 & 0.72 & 0.60 & 0.55 & 0.80 & 0.70 & 0.73 & 0.64 & 0.90 & 0.84 & 0.87 & 0.86 & 0.81 & \\
\hline 0.87 & 0.82 & 0.86 & 0.91 & 0.89 & 0.98 & 0.96 & 0.97 & 0.95 & 0.93 & 0.96 & 0.91 & 0.62 & 0.73 & \\
\hline 0.87 & 0.83 & 0.87 & 0.91 & 0.89 & 0.98 & 0.96 & 0.97 & 0.95 & 0.92 & 0.96 & 0.91 & 0.61 & 0.73 & \\
\hline 0.86 & 0.81 & 0.85 & 0.90 & 0.88 & 0.98 & 0.96 & 0.97 & 0.95 & 0.93 & 0.95 & 0.92 & 0.63 & 0.73 & \\
\hline 0.86 & 0.81 & 0.85 & 0.90 & 0.88 & 0.98 & 0.96 & 0.97 & 0.95 & 0.93 & 0.95 & 0.92 & 0.63 & 0.73 & \\
\hline 0.86 & 0.81 & 0.85 & 0.90 & 0.88 & 0.98 & 0.96 & 0.97 & 0.95 & 0.93 & 0.95 & 0.92 & 0.63 & 0.73 & \\
\hline 0.98 & 0.99 & 0.97 & 0.96 & 0.95 & 0.86 & 0.91 & 0.92 & 0.85 & 0.75 & 0.86 & 0.75 & 0.45 & 0.68 & \\
\hline 0.98 & 0.99 & 0.97 & 0.96 & 0.95 & 0.86 & 0.91 & 0.92 & 0.85 & 0.75 & 0.86 & 0.75 & 0.45 & 0.68 & \\
\hline 0.98 & 0.99 & 0.97 & 0.96 & 0.95 & 0.86 & 0.91 & 0.92 & 0.85 & 0.75 & 0.86 & 0.7 & 0.45 & 0.68 & \\
\hline 0.98 & 0.99 & 0.97 & 0.96 & 0.95 & 0.86 & 0.91 & 0.92 & 0.85 & 0.75 & 0.86 & 0.75 & 0.45 & 0.68 & \\
\hline 0.98 & 0.99 & 0.97 & 0.96 & 0.95 & 0.86 & 0.91 & 0.92 & 0.85 & 0.75 & 0.86 & 0.75 & 0.45 & 0.68 & \\
\hline 0.98 & 0.99 & 0.97 & 0.96 & 0.95 & 0.86 & 0.91 & 0.92 & 0.85 & 0.75 & 0.86 & 0.75 & 0.45 & 0.68 & \\
\hline 0.98 & 0.99 & 0.97 & 0.96 & 0.95 & 0.86 & 0.91 & 0.92 & 0.85 & 0.75 & 0.86 & 0.75 & 0.44 & 0.68 & \\
\hline 0.97 & 0.99 & 0.97 & 0.96 & 0.95 & 0.86 & 0.91 & 0.92 & 0.85 & 0.75 & 0.86 & 0.75 & 0.44 & 0.68 & \\
\hline 0.98 & 0.99 & 0.97 & 0.96 & 0.95 & 0.86 & 0.91 & 0.92 & 0.85 & 0.75 & 0.86 & 0.75 & 0.45 & 0.68 & \\
\hline 0.98 & 0.99 & 0.97 & 0.96 & 0.95 & 0.86 & 0.91 & 0.92 & 0.85 & 0.75 & 0.86 & 0.75 & 0.45 & 0.68 & \\
\hline 0.98 & 0.99 & 0.97 & 0.96 & 0.95 & 0.86 & 0.91 & 0.92 & 0.85 & 0.75 & 0.86 & 0.75 & 0.45 & 0.68 & \\
\hline 0.98 & 0.99 & 0.97 & 0.96 & 0.95 & 0.86 & 0.91 & 0.92 & 0.85 & 0.75 & 0.86 & 0.75 & 0.45 & 0.68 & \\
\hline 0.98 & 0.99 & 0.97 & 0.96 & 0.95 & 0.86 & 0.91 & 0.92 & 0.85 & 0.75 & 0.86 & 0.75 & 0.45 & 0.68 & \\
\hline
\end{tabular}


Case

1261

1262

1263

1264

1265

1266

1267

1268

1269

1270

1271

1272

1273

1274

1275

1276

1277

1278

1279

1280

1281

1282

1283

1284

1285

1286

1287

1288

1289

1290

1291

1292

1293

1294

1295

1296

1297

1298

1299

1300

1301

1302
Experiment

LEU-COMP-THERM-078-014 LEU-COMP-THERM-078-015 LEU-COMP-THERM-079-001 LEU-COMP-THERM-079-002 LEU-COMP-THERM-079-003 LEU-COMP-THERM-079-004 LEU-COMP-THERM-079-005 LEU-COMP-THERM-079-006 LEU-COMP-THERM-079-007 LEU-COMP-THERM-079-008 LEU-COMP-THERM-079-009 EU-COMP-THERM-079-010 LEU-COMP-THERM-080-001 LEU-COMP-THERM-080-002 EU-COMP-THERM-080-003 LEU-COMP-THERM-080-004 LEU-COMP-THERM-080-005 LEU-COMP-THERM-080-006 LEU-COMP-THERM-080-007 LEU-COMP-THERM-080-008 EU-COMP-THERM-080-009 LEU-COMP-THERM-080-010 LEU-COMP-THERM-080-011 EU-COMP-THERM-082-002 LEU-COMP-THERM-082-003 LEU-COMP-THERM-082-004 LEU-COMP-THERM-082-005 LEU-COMP-THERM-082-006 LEU-COMP-THERM-083-001_ EU-COMP-THERM-083-002 LEU-COMP-THERM-083-003 LEU-COMP-THERM-084-001 LEU-COMP-THERM-085-001 LEU-COMP-THERM-085-002 LEU-COMP-THERM-085-003 LEU-COMP-THERM-085-004 LEU-COMP-THERM-085-005 LEU-COMP-THERM-085-006 LEU-COMP-THERM-085-007 LEU-COMP-THERM-085-008 LEU-COMP-THERM-085-009 LEU-COMP-THERM-085-010_-

\begin{tabular}{|c|c|c|c|c|c|c|c|c|c|c|c|c|c|c|}
\hline 1 & 2 & 3 & 4 & 5 & 6 & 7 & 8 & 9 & 10 & 11 & 12 & 13 & 14 & 15 \\
\hline 0.98 & 0.99 & 0.97 & 0.96 & 0.95 & 0.86 & 0.91 & 0.92 & 0.85 & 0.75 & 0.86 & 0.75 & 0.45 & 0.68 & 0.62 \\
\hline 0.97 & 1.00 & 0.97 & 0.97 & 0.96 & 0.86 & 0.92 & 0.92 & 0.86 & 0.74 & 0.86 & 0.74 & 0.43 & 0.66 & .60 \\
\hline 0.99 & 0.96 & 0.98 & 0.93 & 0.91 & 0.87 & 0.89 & 0.91 & 0.82 & 0.79 & 0.88 & 0.78 & 0.50 & 0.71 & .6 \\
\hline 0.99 & 0.96 & 0.98 & 0.93 & 0.91 & 0.87 & 0.89 & 0.91 & 0.81 & 0.79 & 0.88 & 0.78 & 0.50 & 0.71 & 6 \\
\hline 0.99 & 0.96 & 0.98 & 0.93 & 0.91 & 0.87 & 0.89 & 0.91 & 0.81 & 0.80 & 0.89 & 0.79 & 0.50 & 0.71 & 6 \\
\hline 0.99 & 0.96 & 0.98 & 0.93 & 0.91 & 0.87 & 0.89 & 0.91 & 0.81 & 0.80 & 0.89 & 0.79 & 0.51 & 0.71 & 0.6 \\
\hline 0.99 & 0.96 & 0.98 & 0.93 & 0.90 & 0.87 & 0.89 & 0.91 & 0.81 & 0.81 & 0.89 & 0.80 & 0.52 & 0.72 & 0.0 \\
\hline 0.96 & 0.96 & 0.95 & 0.95 & 0.95 & 0.88 & 0.92 & 0.91 & 0.86 & 0.78 & 0.88 & 0.77 & 0.43 & 0.63 & \\
\hline 0.96 & 0.96 & 0.95 & 0.95 & 0.94 & 0.88 & 0.92 & 0.91 & 0.86 & 0.78 & 0.88 & 0.77 & 0.43 & 0.63 & \\
\hline 0.96 & 0.96 & 0.95 & 0.95 & 0.94 & 0.89 & 0.92 & 0.91 & 0.86 & 0.79 & 0.88 & 0.78 & 0.44 & 0.64 & \\
\hline 0.96 & 0.96 & 0.95 & 0.94 & 0.94 & 0.89 & 0.92 & 0.91 & 0.86 & 0.79 & 0.88 & 0.78 & 0.44 & 0.64 & \\
\hline 0.96 & 0.95 & 0.95 & 0.94 & 0.93 & 0.89 & 0.91 & 0.91 & 0.86 & 0.81 & 0.89 & 0.79 & 0.46 & 0.65 & \\
\hline 0.98 & 0.98 & 0.98 & 0.94 & 0.93 & 0.86 & 0.89 & 0.91 & 0.83 & 0.76 & 0.87 & 0.76 & 0.48 & 0.71 & \\
\hline 0.98 & 0.98 & 0.98 & 0.94 & 0.93 & 0.86 & 0.90 & 0.91 & 0.83 & 0.76 & 0.87 & 0.76 & 0.48 & 0.71 & \\
\hline 0.98 & 0.98 & 0.98 & 0.94 & 0.93 & 0.86 & 0.90 & 0.91 & 0.83 & 0.76 & 0.87 & 0.76 & 0.48 & 0.71 & \\
\hline 0.98 & 0.98 & 0.98 & 0.94 & 0.93 & 0.86 & 0.90 & 0.91 & 0.83 & 0.76 & 0.87 & 0.76 & 0.48 & 0.71 & \\
\hline 0.98 & 0.98 & 0.98 & 0.94 & 0.93 & 0.86 & 0.90 & 0.91 & 0.83 & 0.76 & 0.87 & 0.76 & 0.48 & 0.71 & \\
\hline 0.98 & 0.98 & 0.98 & 0.94 & 0.93 & 0.86 & 0.90 & 0.91 & 0.83 & 0.76 & 0.87 & 0.76 & 0.48 & 0.71 & \\
\hline 0.98 & 0.98 & 0.98 & 0.94 & 0.93 & 0.86 & 0.90 & 0.91 & 0.83 & 0.76 & 0.87 & 0.76 & 0.48 & 0.71 & \\
\hline 0.98 & 0.98 & 0.98 & 0.94 & 0.93 & 0.86 & 0.90 & 0.91 & 0.83 & 0.76 & 0.87 & 0.76 & 0.48 & 0.71 & \\
\hline 0.98 & 0.98 & 0.98 & 0.94 & 0.93 & 0.86 & 0.90 & 0.91 & 0.83 & 0.76 & 0.87 & 0.76 & 0.48 & 0.71 & \\
\hline 0.98 & 0.98 & 0.98 & 0.94 & 0.93 & 0.86 & 0.90 & 0.91 & 0.83 & 0.76 & 0.87 & 0.76 & 0.48 & 0.71 & \\
\hline 0.98 & 0.99 & 0.98 & 0.96 & 0.95 & 0.86 & 0.91 & 0.92 & 0.85 & 0.75 & 0.86 & 0.75 & 0.46 & 0.69 & \\
\hline 0.88 & 0.84 & 0.87 & 0.91 & 0.90 & 0.98 & 0.96 & 0.97 & 0.95 & 0.92 & 0.96 & 0.91 & 0.61 & 0.72 & \\
\hline 0.87 & 0.82 & 0.86 & 0.90 & 0.88 & 0.98 & 0.96 & 0.96 & 0.95 & 0.93 & 0.96 & 0.92 & 0.63 & 0.73 & \\
\hline 0.87 & 0.82 & 0.86 & 0.90 & 0.88 & 0.98 & 0.96 & 0.97 & 0.95 & 0.93 & 0.96 & 0.92 & 0.63 & 0.73 & \\
\hline 0.87 & 0.82 & 0.86 & 0.90 & 0.88 & 0.98 & 0.96 & 0.96 & 0.95 & 0.93 & 0.96 & 0.92 & 0.63 & 0.73 & \\
\hline 0.87 & 0.81 & 0.86 & 0.90 & 0.88 & 0.98 & 0.96 & 0.96 & 0.94 & 0.94 & 0.96 & 0.92 & 0.63 & 0.73 & \\
\hline 0.88 & 0.83 & 0.87 & 0.91 & 0.89 & 0.98 & 0.96 & 0.97 & 0.95 & 0.93 & 0.96 & 0.91 & 0.62 & 0.73 & \\
\hline 0.88 & 0.83 & 0.87 & 0.91 & 0.89 & 0.98 & 0.96 & 0.97 & 0.95 & 0.92 & 0.96 & 0.91 & 0.62 & 0.72 & \\
\hline 0.87 & 0.83 & 0.87 & 0.91 & 0.89 & 0.98 & 0.96 & 0.97 & 0.95 & 0.93 & 0.96 & 0.91 & 0.62 & 0.72 & \\
\hline 0.84 & 0.79 & 0.84 & 0.88 & 0.86 & 0.98 & 0.95 & 0.96 & 0.94 & 0.93 & 0.95 & 0.91 & 0.63 & 0.72 & \\
\hline 0.98 & 0.97 & 0.98 & 0.94 & 0.92 & 0.87 & 0.90 & 0.92 & 0.83 & 0.79 & 0.88 & 0.78 & 0.50 & 0.72 & \\
\hline 0.98 & 0.97 & 0.98 & 0.94 & 0.92 & 0.87 & 0.90 & 0.92 & 0.83 & 0.79 & 0.89 & 0.79 & 0.51 & 0.73 & \\
\hline 0.99 & 0.97 & 0.98 & 0.94 & 0.92 & 0.87 & 0.90 & 0.92 & 0.83 & 0.79 & 0.89 & 0.79 & 0.51 & 0.73 & 0. \\
\hline 0.98 & 0.97 & 0.98 & 0.94 & 0.92 & 0.87 & 0.90 & 0.92 & 0.84 & 0.79 & 0.88 & 0.78 & 0.50 & 0.72 & 0. \\
\hline 0.98 & 0.97 & 0.98 & 0.94 & 0.92 & 0.87 & 0.90 & 0.92 & 0.83 & 0.79 & 0.88 & 0.78 & 0.50 & 0.72 & \\
\hline 0.98 & 0.98 & 0.98 & 0.94 & 0.93 & 0.86 & 0.90 & 0.92 & 0.84 & 0.78 & 0.88 & 0.78 & 0.49 & 0.71 & \\
\hline 0.99 & 0.98 & 0.98 & 0.94 & 0.93 & 0.87 & 0.90 & 0.92 & 0.84 & 0.78 & 0.88 & 0.78 & 0.49 & 0.72 & \\
\hline 0.99 & 0.98 & 0.98 & 0.95 & 0.93 & 0.87 & 0.90 & 0.92 & 0.84 & 0.78 & 0.88 & 0.78 & 0.49 & 0.71 & 0. \\
\hline 0.99 & 0.98 & 0.98 & 0.95 & 0.94 & 0.87 & 0.91 & 0.92 & 0.84 & 0.78 & 0.88 & 0.78 & 0.49 & 0.71 & \\
\hline 0.99 & 0.98 & 0.99 & 0.95 & 0.94 & 0.87 & 0.91 & 0.92 & 0.84 & 0.78 & 0.89 & 0.78 & 0.49 & 0.71 & \\
\hline
\end{tabular}


Experiment

LEU-COMP-THERM-085-011 LEU-COMP-THERM-085-012 LEU-COMP-THERM-085-013 LEU-COMP-THERM-089-001 LEU-COMP-THERM-089-002

LEU-COMP-THERM-089-003 LEU-COMP-THERM-089-004 LEU-COMP-THERM-090-001 LEU-COMP-THERM-090-002 LEU-COMP-THERM-090-003 LEU-COMP-THERM-090-004 LEU-COMP-THERM-090-005 LEU-COMP-THERM-090-006 LEU-COMP-THERM-090-007 LEU-COMP-THERM-090-008 LEU-COMP-THERM-090-009 LEU-COMP-THERM-091-001 LEU-COMP-THERM-091-002 LEU-COMP-THERM-091-003 LEU-COMP-THERM-091-004 LEU-COMP-THERM-091-005 LEU-COMP-THERM-091-006 LEU-COMP-THERM-091-007 LEU-COMP-THERM-091-008 LEU-COMP-THERM-091-009 LEU-COMP-THERM-092-001 LEU-COMP-THERM-092-002 LEU-COMP-THERM-092-003 LEU-COMP-THERM-092-004 LEU-COMP-THERM-092-005 LEU-COMP-THERM-092-006 LEU-COMP-THERM-094-001 LEU-COMP-THERM-094-002 LEU-COMP-THERM-094-003 LEU-COMP-THERM-094-004 LEU-COMP-THERM-094-005 LEU-COMP-THERM-094-006 LEU-COMP-THERM-094-007 LEU-COMP-THERM-094-008 LEU-COMP-THERM-094-009 LEU-COMP-THERM-094-010 LEU-COMP-THERM-094-011_

\begin{tabular}{|c|c|c|c|c|c|c|c|c|c|c|c|c|c|c|}
\hline 1 & 2 & 3 & 4 & 5 & 6 & 7 & 8 & 9 & 10 & 11 & 12 & 13 & 14 & 15 \\
\hline 0.99 & 0.98 & 0.98 & 0.95 & 0.94 & 0.87 & 0.91 & 0.92 & 0.84 & 0.78 & 0.88 & 0.78 & 0.49 & 0.71 & 0.64 \\
\hline 0.99 & 0.98 & 0.99 & 0.95 & 0.94 & 0.88 & 0.91 & 0.92 & 0.85 & 0.79 & 0.89 & 0.79 & 0.50 & 0.71 & .6 \\
\hline 0.99 & 0.98 & 0.98 & 0.95 & 0.94 & 0.87 & 0.91 & 0.92 & 0.85 & 0.78 & 0.89 & 0.78 & 0.49 & 0.71 & \\
\hline 0.88 & 0.83 & 0.87 & 0.91 & 0.89 & 0.98 & 0.96 & 0.97 & 0.95 & 0.93 & 0.96 & 0.91 & 0.62 & 0.73 & \\
\hline 0.87 & 0.82 & 0.87 & 0.90 & 0.88 & 0.98 & 0.96 & 0.97 & 0.95 & 0.94 & 0.96 & 0.92 & 0.63 & 0.73 & \\
\hline 0.87 & 0.83 & 0.87 & 0.91 & 0.89 & 0.98 & 0.96 & 0.97 & 0.95 & 0.93 & 0.96 & 0.92 & 0.63 & 0.73 & \\
\hline 0.88 & 0.83 & 0.87 & 0.91 & 0.89 & 0.98 & 0.96 & 0.97 & 0.95 & 0.93 & 0.96 & 0.92 & 0.62 & 0.73 & \\
\hline 0.85 & 0.80 & 0.84 & 0.89 & 0.87 & 0.98 & 0.95 & 0.96 & 0.95 & 0.92 & 0.95 & 0.91 & 0.62 & 0.71 & \\
\hline 0.84 & 0.80 & 0.84 & 0.89 & 0.87 & 0.98 & 0.95 & 0.96 & 0.94 & 0.93 & 0.95 & 0.91 & 0.62 & 0.72 & \\
\hline 0.85 & 0.80 & 0.84 & 0.89 & 0.87 & 0.98 & 0.95 & 0.96 & 0.94 & 0.93 & 0.95 & 0.91 & 0.63 & 0.72 & \\
\hline 0.85 & 0.80 & 0.85 & 0.89 & 0.87 & 0.98 & 0.95 & 0.96 & 0.94 & 0.93 & 0.95 & 0.92 & 0.63 & 0.72 & \\
\hline 0.85 & 0.80 & 0.85 & 0.89 & 0.87 & 0.98 & 0.95 & 0.96 & 0.95 & 0.93 & 0.95 & 0.91 & 0.63 & 0.72 & \\
\hline 0.85 & 0.80 & 0.84 & 0.89 & 0.87 & 0.98 & 0.95 & 0.96 & 0.94 & 0.93 & 0.95 & 0.91 & 0.63 & 0.72 & \\
\hline 0.84 & 0.79 & 0.84 & 0.89 & 0.87 & 0.98 & 0.95 & 0.96 & 0.94 & 0.93 & 95 & 0.91 & 0.63 & 0.72 & \\
\hline 0.85 & 0.80 & 0.85 & 0.89 & 0.87 & 0.98 & 0.95 & 0.96 & 0.95 & 0.93 & 0.95 & 0.91 & 0.62 & 0.72 & \\
\hline 0.86 & 0.81 & 0.86 & 0.90 & 0.88 & 0.98 & 0.96 & 0.96 & 0.95 & 0.93 & 0.95 & 0.91 & 0.62 & 0.72 & \\
\hline 0.87 & 0.81 & 0.86 & 0.90 & 0.88 & 0.98 & 0.96 & 0.96 & 0.95 & 0.93 & 0.96 & 0.92 & 0.63 & 0.73 & \\
\hline 0.88 & 0.83 & 0.87 & 0.91 & 0.89 & 0.99 & 0.96 & 0.97 & 0.95 & 0.93 & 0.96 & 0.92 & 0.62 & 0.73 & \\
\hline 0.87 & 0.83 & 0.87 & 0.91 & 0.89 & 0.99 & 0.96 & 0.97 & 0.95 & 0.93 & .96 & 0.92 & 0.63 & 0.73 & \\
\hline 0.87 & 0.82 & 0.86 & 0.90 & 0.88 & 0.98 & 0.96 & 0.97 & 0.95 & 0.93 & .96 & 0.92 & 0.63 & 0.73 & \\
\hline 0.87 & 0.82 & 0.86 & 0.90 & 0.88 & 0.98 & 0.96 & 0.97 & 0.95 & 0.93 & 0.96 & 0.92 & 0.63 & 0.73 & \\
\hline 0.86 & 0.81 & 0.85 & 0.89 & 0.87 & 0.98 & 0.95 & 0.96 & 0.94 & 0.93 & 0.96 & 0.92 & 0.62 & 0.72 & \\
\hline 0.87 & 0.82 & 0.86 & 0.90 & 0.88 & 0.98 & 0.96 & 0.96 & 0.95 & 0.93 & 0.96 & 0.92 & 0.62 & 0.72 & \\
\hline 0.87 & 0.82 & 0.86 & 0.90 & 0.88 & 0.98 & 0.96 & 0.96 & 0.95 & 0.93 & 0.96 & 0.92 & 0.62 & 0.71 & \\
\hline 0.87 & 0.82 & 0.86 & 0.91 & 0.89 & 0.98 & 0.96 & 0.96 & 0.95 & 0.93 & 0.96 & 0.91 & 0.61 & 0.71 & \\
\hline 0.87 & 0.83 & 0.87 & 0.91 & 0.89 & 0.98 & 0.96 & 0.97 & 0.95 & 0.93 & 0.96 & 0.91 & 0.62 & 0.73 & \\
\hline 0.87 & 0.83 & 0.87 & 0.91 & 0.89 & 0.98 & 0.96 & 0.97 & 0.95 & 0.93 & 0.96 & 0.91 & 0.62 & 0.73 & \\
\hline 0.87 & 0.83 & 0.87 & 0.91 & 0.89 & 0.98 & 0.96 & 0.97 & 0.95 & 0.93 & 0.96 & 0.91 & 0.62 & 0.73 & \\
\hline 0.87 & 0.83 & 0.87 & 0.91 & 0.89 & 0.98 & 0.96 & 0.97 & 0.95 & 0.93 & 0.96 & 0.91 & 0.62 & 0.73 & \\
\hline 0.87 & 0.82 & 0.87 & 0.90 & 0.88 & 0.98 & 0.96 & 0.97 & 0.95 & 0.93 & 0.96 & 0.92 & 0.62 & 0.73 & \\
\hline 0.88 & 0.83 & 0.87 & 0.91 & 0.89 & 0.98 & 0.96 & 0.97 & 0.95 & 0.93 & 0.96 & 0.92 & 0.62 & 0.73 & \\
\hline 0.90 & 0.80 & 0.90 & 0.76 & 0.73 & 0.80 & 0.77 & 0.81 & 0.69 & 0.82 & 0.8 & 0. & 0.71 & 0.82 & \\
\hline 0.90 & 0.79 & 0.90 & 0.76 & 0.72 & 0.79 & 0.76 & 0.81 & 0.68 & 0.82 & 0.85 & 0.81 & 0.71 & 0.82 & \\
\hline 0.91 & 0.81 & 0.91 & 0.78 & 0.74 & 0.80 & 0.78 & 0.82 & 0.69 & 0.81 & 0.85 & 0.80 & 0.69 & 0.81 & \\
\hline 0.91 & 0.82 & 0.91 & 0.78 & 0.74 & 0.80 & 0.78 & 0.82 & 0.70 & 0.82 & 0.85 & 0.81 & 0.69 & 0.81 & \\
\hline 0.92 & 0.83 & 0.92 & 0.79 & 0.75 & 0.80 & 0.78 & 0.83 & 0.70 & 0.81 & 0.85 & 0.80 & 0.68 & 0.81 & \\
\hline 0.93 & 0.84 & 0.93 & 0.80 & 0.77 & 0.81 & 0.79 & 0.84 & 0.71 & 0.81 & 0.86 & 0.80 & 0.67 & 0.81 & \\
\hline 0.93 & 0.85 & 0.93 & 0.81 & 0.77 & 0.81 & 0.80 & 0.84 & 0.71 & 0.80 & 0.85 & 0.80 & 0.66 & 0.80 & \\
\hline 0.93 & 0.85 & 0.93 & 0.81 & 0.78 & 0.81 & 0.80 & 0.84 & 0.72 & 0.80 & 0.86 & 0.80 & 0.65 & 0.80 & \\
\hline 0.93 & 0.85 & 0.93 & 0.81 & 0.78 & 0.81 & 0.80 & 0.84 & 0.72 & 0.80 & 0.86 & 0.80 & 0.66 & 0.80 & \\
\hline 0.94 & 0.86 & 0.94 & 0.82 & 0.78 & 0.81 & 0.81 & 0.84 & 0.72 & 0.81 & 0.86 & 0.80 & 0.66 & 0.80 & \\
\hline 0.94 & 0.86 & 0.94 & 0.82 & 0.79 & 0.82 & 0.81 & 0.85 & 0.73 & 0.81 & 0.86 & 0.80 & 0.65 & 0.80 & \\
\hline
\end{tabular}
5 0.64 .57 57 0.55 55 0.56 .57 57
.57 .56 55 .57 0.57 0.71 .71 .57 0.55
0.55
0.56 .56 .56 .56 0.56
0.56 0.56
0.55 0.55 .57 57
57 .57 70 71
.70 .71 
1368

1369

1370

1371

1372

1373

1374

1375

1376

1377

1378

1379

1380

1381

1382

1383

1384

1385

1386

\section{Experiment}

LEU-MET-THERM-001-001_E LEU-MET-THERM-002-001_E LEU-MET-THERM-002-002 E LEU-MET-THERM-002-003_E LEU-MET-THERM-002-004 E

LEU-MET-THERM-002-005 E LEU-MET-THERM-002-006_E LEU-MET-THERM-002-007 E

EU-MET-THERM-002-008 LEU-MET-THERM-002-009_E LEU-MET-THERM-002-010_E

EU-MET-THERM-002-011 E LEU-MET-THERM-002-012_E LEU-MET-THERM-004-001 E

EU-MET-THERM-004-002 E LEU-MET-THERM-004-003_E LEU-MET-THERM-004-004 E

EU-MET-THERM-004-005 E LEU-MET-THERM-004-006_E LEU-MET-THERM-004-007 E

EEU-MET-THERM-004-008 E LEU-MET-THERM-006-001_E LEU-MET-THERM-006-002 E

EU-MET-THERM-006-003 LEU-MET-THERM-006-004_E LEU-MET-THERM-006-005_E EU-MET-THERM-006-006 LEU-MET-THERM-006-007_E LEU-MET-THERM-006-008_E LEU-MET-THERM-006-009 E LEU-MET-THERM-006-010_E LEU-MET-THERM-006-011_E EU-MET-THERM-006-012 LEU-MET-THERM-006-013_E LEU-MET-THERM-006-014 E LEU-MET-THERM-006-015 E LEU-MET-THERM-006-016_E LEU-MET-THERM-006-017 E LEU-MET-THERM-006-018 E LEU-MET-THERM-006-019_E LEU-MET-THERM-006-020_E LEU-MET-THERM-006-021_E

\begin{tabular}{|c|c|c|c|c|c|c|c|c|c|c|c|c|c|c|}
\hline 1 & 2 & 3 & 4 & 5 & 6 & 7 & 8 & 9 & 10 & 11 & 12 & 13 & 14 & 15 \\
\hline 0.41 & 0.22 & 0.38 & 0.23 & 0.19 & 0.44 & 0.33 & 0.33 & 0.24 & 0.55 & 0.46 & 0.50 & 0.55 & 0.43 & 0.22 \\
\hline .52 & 0.40 & 0.51 & 0.38 & 0.36 & 0.51 & 0.45 & 0.46 & 0.40 & 0.58 & 0.54 & 0.56 & 0.43 & 0.41 & 0.26 \\
\hline .50 & 0.39 & 0.49 & 0.37 & 0.35 & 0.50 & 0.44 & 0.44 & 0.39 & 0.55 & 0.52 & 0.53 & 0.41 & 0.39 & 0.26 \\
\hline 51 & 0.40 & 0.50 & 0.38 & 0.36 & 0.51 & 0.45 & 0.45 & 0.40 & 0.57 & 0.54 & 0.55 & 0.42 & 0.40 & 0.26 \\
\hline 51 & 0.41 & 0.50 & 0.39 & 0.37 & 0.51 & 0.45 & 0.46 & 0.40 & 0.56 & 0.53 & 0.54 & 0.43 & 0.41 & 0.27 \\
\hline 55 & 0.42 & 0.54 & 0.41 & 0.39 & 0.55 & 0.48 & 0.49 & 0.43 & 0.62 & 0.58 & 0.59 & 0.46 & 0.43 & 0.28 \\
\hline 53 & 0.40 & 0.52 & 0.39 & 0.36 & 0.53 & 0.47 & 0.47 & 0.40 & 0.60 & 0.56 & 0.57 & 0.50 & .46 & 0.30 \\
\hline 55 & 0.43 & 0.54 & 0.41 & 0.39 & 0.55 & 0.49 & 0.49 & 0.43 & 0.61 & 0.58 & 0.59 & 0.46 & 0.44 & 0.28 \\
\hline 50 & 0.40 & 0.49 & 0.38 & 0.36 & 0.50 & 0.44 & 0.45 & 0.39 & 0.54 & 0.52 & 0.52 & 0.41 & 0.40 & 0.26 \\
\hline 52 & 0.41 & 0.51 & 0.39 & 0.37 & 0.52 & 0.46 & 0.46 & 0.41 & 0.57 & 0.54 & 0.55 & 0.43 & 0.41 & 0.27 \\
\hline 58 & 0.45 & 0.57 & 0.44 & 0.42 & 0.58 & 0.51 & 0.51 & 0.45 & 0.64 & 0.60 & 0.61 & 0.48 & .46 & 0.30 \\
\hline 57 & 0.45 & 0.55 & 0.43 & 0.41 & 0.56 & 0.50 & 0.50 & 0.44 & 0.62 & 0.59 & 0.59 & 0.47 & .45 & 0.30 \\
\hline 59 & .45 & 0.58 & 0.44 & 0.42 & 0.59 & 0.52 & 0.52 & 0.46 & 0.67 & 0.63 & 0.64 & 0.49 & 0.47 & 0.30 \\
\hline 93 & .87 & 0.93 & 0.84 & 0.80 & 0.78 & 0.79 & 0.84 & 0.71 & 0.76 & 0.83 & 0.75 & 0.50 & 0.71 & 0.63 \\
\hline 93 & .87 & 0.93 & 0.84 & 0.80 & 0.78 & 0.78 & 0.84 & 0.70 & 0.75 & 0.82 & 0.74 & 0.48 & 0.70 & 0.63 \\
\hline 95 & 0.90 & 0.94 & 0.87 & 0.84 & 0.81 & 0.82 & 0.86 & 0.74 & 0.77 & 0.85 & 0.76 & 0.48 & .69 & 0.62 \\
\hline 95 & 0.90 & 0.94 & 0.87 & 0.84 & 0.81 & 0.81 & 0.86 & 0.74 & 0.76 & 0.84 & 0.75 & 0.47 & .69 & 0.61 \\
\hline 95 & 0.90 & 0.95 & 0.87 & 0.84 & 0.83 & 0.83 & 0.87 & 0.76 & 0.80 & 0.87 & 0.78 & 0.49 & 0.69 & 0.60 \\
\hline 96 & 0.90 & 0.95 & 0.88 & 0.85 & 0.83 & 0.83 & 0.87 & 0.76 & 0.79 & 0.86 & 0.78 & 0.48 & 0.69 & 0.60 \\
\hline 92 & 0.83 & 0.91 & 0.82 & 0.80 & 0.83 & 0.81 & 0.84 & 0.74 & 0.83 & 0.87 & 0.81 & 0.52 & 0.67 & 0.56 \\
\hline .92 & 0.84 & 0.91 & 0.82 & 0.80 & 0.83 & 0.81 & 0.84 & 0.74 & 0.83 & 0.87 & 0.81 & .52 & .67 & 0.56 \\
\hline 48 & 0.24 & 0.46 & 0.27 & 0.22 & 0.51 & 0.37 & 0.39 & 0.28 & 0.68 & 0.56 & 0.63 & 0.69 & .54 & 0.31 \\
\hline 46 & 0.23 & 0.44 & 0.25 & 0.20 & 0.50 & 0.35 & 0.37 & 0.27 & 0.67 & 0.55 & 0.62 & 0.69 & 0.53 & 0.30 \\
\hline 48 & 0.24 & 0.45 & 0.27 & 0.21 & 0.51 & 0.37 & 0.39 & 0.28 & 0.68 & 0.56 & 0.63 & 0.69 & 0.54 & 0.31 \\
\hline .57 & 0.34 & 0.54 & 0.36 & 0.31 & 0.58 & 0.45 & 0.47 & 0.36 & 0.73 & 0.63 & 0.68 & 70 & 59 & 0.35 \\
\hline 58 & 0.35 & 0.55 & 0.37 & 0.31 & 0.58 & 0.45 & 0.48 & 0.36 & 0.73 & 0.63 & 0.68 & .69 & 0.59 & 0.36 \\
\hline 63 & 0.41 & 0.60 & 0.43 & 0.38 & 0.63 & 0.51 & 0.53 & 0.41 & 0.77 & 0.68 & 0.71 & 0.69 & 0.61 & 0.39 \\
\hline 65 & 0.43 & 0.62 & 0.45 & 0.39 & 0.64 & 0.52 & 0.55 & 0.42 & 0.77 & 0.69 & 0.72 & 0.69 & 0.62 & 0.40 \\
\hline 63 & 0.41 & 0.60 & 0.43 & 0.37 & 0.63 & 0.50 & 0.53 & 0.41 & 0.76 & 0.68 & 0.71 & 0.69 & 0.61 & 0.38 \\
\hline 64 & 0.42 & 0.61 & 0.44 & 0.38 & 0.64 & 0.51 & 0.54 & 0.42 & 0.77 & 0.68 & 0.72 & 0.69 & 0.62 & 0.39 \\
\hline 64 & 0.42 & 0.61 & 0.44 & 0.39 & 0.64 & 0.51 & 0.54 & 0.42 & 0.77 & 0.69 & 0.72 & 0.69 & 0.62 & 0.39 \\
\hline 62 & 0.40 & 0.59 & 0.42 & 0.36 & 0.62 & 0.49 & 0.52 & 0.40 & 0.76 & 0.67 & 0.71 & 0.69 & 0.61 & 0.38 \\
\hline 64 & 0.42 & 0.61 & 0.44 & 0.39 & 0.64 & 0.52 & 0.54 & 0.42 & 0.77 & 0.69 & 0.72 & 0.68 & 0.61 & 0.38 \\
\hline 65 & 0.43 & 0.62 & 0.45 & 0.40 & 0.65 & 0.52 & 0.55 & 0.43 & 0.77 & 0.69 & 0.72 & 0.68 & 0.61 & 0.39 \\
\hline 64 & 0.42 & 0.61 & 0.45 & 0.40 & 0.64 & 0.52 & 0.54 & 0.43 & 0.77 & 0.69 & 0.72 & 0.67 & 0.60 & 0.38 \\
\hline .62 & 0.40 & 0.59 & 0.43 & 0.37 & 0.63 & 0.50 & 0.52 & 0.41 & 0.76 & 0.68 & 0.71 & 0.67 & 0.59 & 0.36 \\
\hline 62 & 0.41 & 0.59 & 0.43 & 0.38 & 0.63 & 0.50 & 0.53 & 0.41 & 0.76 & 0.68 & 0.71 & 0.67 & 0.59 & 0.37 \\
\hline & 0.36 & 0.55 & 0.39 & 0.34 & 0.61 & 0.47 & 0.49 & 0.39 & 0.75 & 0.65 & 0.69 & 0.65 & 0.56 & 0.34 \\
\hline 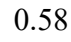 & 0.36 & 0.55 & 0.39 & 0.34 & 0.60 & 0.47 & 0.49 & 0.39 & 0.74 & 0.65 & 0.69 & 0.65 & 0.56 & 0.33 \\
\hline .47 & 0.23 & 0.45 & 0.26 & 0.21 & 0.51 & 0.36 & 0.38 & 0.28 & 0.68 & 0.56 & 0.62 & 0.68 & 0.53 & 0.30 \\
\hline .49 & 0.25 & 0.46 & 0.28 & 0.22 & 0.52 & 0.37 & 0.40 & 0.29 & 0.68 & 0.57 & 0.63 & 0.68 & 0.54 & 0.31 \\
\hline 0.57 & 0.35 & 0.55 & 0.37 & 0.32 & 0.59 & 0.45 & 0.48 & 0.37 & 0.74 & 0.64 & 0.68 & 0.69 & 0.58 & 0.35 \\
\hline
\end{tabular}


Case

1387

1388

1389

1390

1391

1392

1393

1394

1395

1396

1397

1398

1399

1400

1401

1402

1403

1404

1405

1406

1407

1408

1409

1410

1411

1412

1413

1414

1415

1416

1417

1418

1419

1420

1421

1422

1423

1424

1425

1426

1427

1428
Experiment

LEU-MET-THERM-006-022_E LEU-MET-THERM-006-023 E LEU-MET-THERM-006-024 E LEU-MET-THERM-006-025_E LEU-MET-THERM-006-026_E

LEU-MET-THERM-006-027 E LEU-MET-THERM-006-028_E LEU-MET-THERM-006-029 E

LEU-MET-THERM-006-030 E LEU-MET-THERM-007-001_E LEU-MET-THERM-007-002 E

EU-MET-THERM-007-003 E LEU-MET-THERM-007-004_E LEU-MET-THERM-007-005 E

EU-MET-THERM-007-006 LEU-MET-THERM-015-001_E LEU-MET-THERM-015-002 E

EU-MET-THERM-015-003 E LEU-MET-THERM-015-004_E LEU-MET-THERM-015-005 E

EU-MET-THERM-015-006 E LEU-MET-THERM-015-007_E LEU-MET-THERM-015-008 E

EU-MET-THERM-015-009 E LEU-MET-THERM-015-010_E LEU-MET-THERM-015-011_E EU-MET-THERM-015-012 E LEU-MET-THERM-015-013_E LEU-MET-THERM-015-014 E EU-MET-THERM-015-015 E LEU-MET-THERM-015-016_E LEU-MET-THERM-015-017_E EU-MET-THERM-015-018 LEU-MET-THERM-015-019_E LEU-MET-THERM-015-020 E EU-MET-THERM-015-021 LEU-MET-THERM-015-022_E LEU-MISC-THERM-001-001 LEU-MISC-THERM-001-002 LEU-MISC-THERM-001-003 LEU-MISC-THERM-001-004 LEU-MISC-THERM-001-005_ $\begin{array}{ll}\mathbf{1} & \mathbf{2} \\ 0.55 & 0.32\end{array}$

$\begin{array}{llll}0.55 & 0.32 & 0.52 & 0.34\end{array}$

$0.55 \quad 0.32$

$0.58 \quad 0.35$

$0.58 \quad 0.36$

$0.59 \quad 0.37$

$\begin{array}{lll}0.57 & 0.35 & 0.57\end{array}$

$\begin{array}{llll}0.57 & 0.35 & 0.54 & 0.38 \\ 0.56 & 0.34 & 0.53 & 0.36\end{array}$

$\begin{array}{lllllllllllllllll}0.97 & 0.98 & 0.97 & 0.95 & 0.93 & 0.85 & 0.89 & 0.91 & 0.82 & 0.75 & 0.86 & 0.74 & 0.43 & 0.67 & 0.60\end{array}$

$\begin{array}{lllllllllllllll}0.97 & 0.99 & 0.96 & 0.96 & 0.96 & 0.86 & 0.91 & 0.92 & 0.85 & 0.75 & 0.86 & 0.74 & 0.41 & 0.64 & 0.58\end{array}$

$\begin{array}{lllllllllllllllll}0.96 & 0.98 & 0.95 & 0.96 & 0.96 & 0.87 & 0.91 & 0.91 & 0.86 & 0.76 & 0.86 & 0.75 & 0.41 & 0.63 & 0.56\end{array}$

$\begin{array}{lllllllllllllllll}0.94 & 0.94 & 0.93 & 0.93 & 0.93 & 0.87 & 0.90 & 0.89 & 0.85 & 0.78 & 0.87 & 0.76 & 0.42 & 0.61 & 0.53\end{array}$

$\begin{array}{lllllllllllllll}0.88 & 0.84 & 0.87 & 0.85 & 0.84 & 0.85 & 0.84 & 0.83 & 0.79 & 0.80 & 0.85 & 0.78 & 0.46 & 0.60 & 0.48\end{array}$

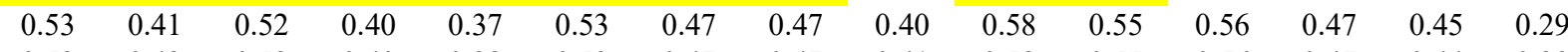

$\begin{array}{lllllllllllllll}0.53 & 0.42 & 0.52 & 0.40 & 0.38 & 0.53 & 0.47 & 0.47 & 0.41 & 0.58 & 0.55 & 0.56 & 0.47 & 0.44 & 0.29\end{array}$

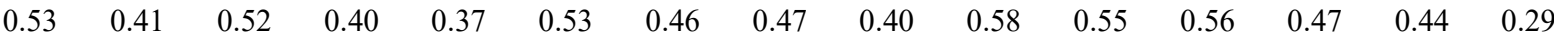

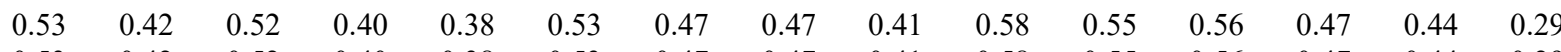

$\begin{array}{lllllllllllllll}0.53 & 0.42 & 0.52 & 0.40 & 0.38 & 0.53 & 0.47 & 0.47 & 0.41 & 0.58 & 0.55 & 0.56 & 0.47 & 0.44 & 0.29\end{array}$

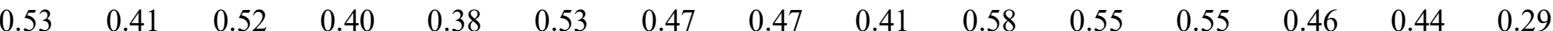

$\begin{array}{lllllllllllllll}0.53 & 0.41 & 0.51 & 0.40 & 0.38 & 0.52 & 0.46 & 0.47 & 0.41 & 0.57 & 0.54 & 0.55 & 0.46 & 0.44 & 0.29\end{array}$

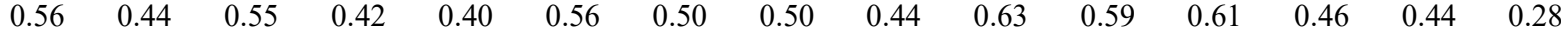

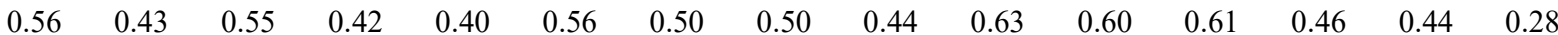

$\begin{array}{lllllllllllllll}0.56 & 0.43 & 0.55 & 0.42 & 0.40 & 0.56 & 0.49 & 0.50 & 0.44 & 0.64 & 0.60 & 0.61 & 0.46 & 0.44 & 0.28\end{array}$

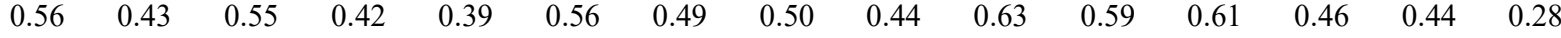

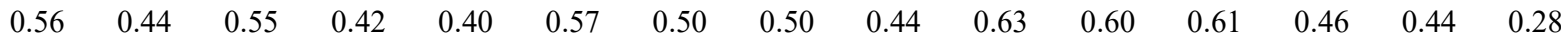

$\begin{array}{lllllllllllllll}0.56 & 0.43 & 0.55 & 0.41 & 0.39 & 0.56 & 0.49 & 0.49 & 0.43 & 0.63 & 0.59 & 0.60 & 0.46 & 0.44 & 0.28\end{array}$

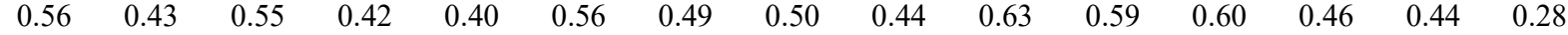

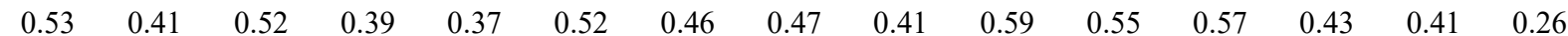

$\begin{array}{lllllllllllllll}0.53 & 0.41 & 0.52 & 0.39 & 0.37 & 0.53 & 0.46 & 0.47 & 0.41 & 0.59 & 0.55 & 0.57 & 0.43 & 0.41 & 0.27\end{array}$

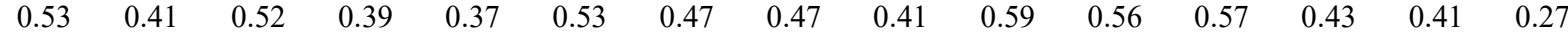

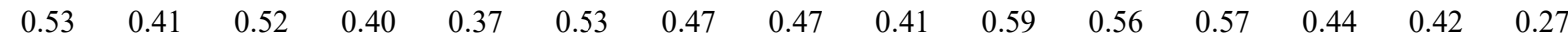

$\begin{array}{lllllllllllllll}0.53 & 0.42 & 0.52 & 0.40 & 0.38 & 0.53 & 0.47 & 0.47 & 0.42 & 0.60 & 0.56 & 0.57 & 0.44 & 0.42 & 0.27\end{array}$

$\begin{array}{lllllllllllllll}0.54 & 0.42 & 0.53 & 0.40 & 0.38 & 0.54 & 0.47 & 0.48 & 0.42 & 0.60 & 0.56 & 0.58 & 0.44 & 0.42 & 0.27\end{array}$

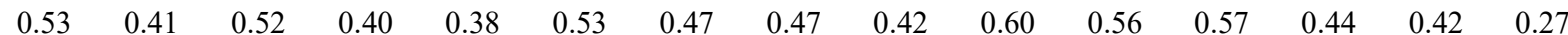

$\begin{array}{lllllllllllllll}0.53 & 0.41 & 0.52 & 0.39 & 0.37 & 0.53 & 0.47 & 0.47 & 0.41 & 0.59 & 0.56 & 0.57 & 0.43 & 0.41 & 0.27\end{array}$

$\begin{array}{llllllllllllllll}0.93 & 0.93 & 0.93 & 0.93 & 0.93 & 0.90 & 0.92 & 0.90 & 0.87 & 0.80 & 0.89 & 0.79 & 0.46 & 0.64 & 0.53\end{array}$

$\begin{array}{llllllllllllllll}0.93 & 0.93 & 0.93 & 0.93 & 0.93 & 0.89 & 0.92 & 0.90 & 0.87 & 0.80 & 0.88 & 0.79 & 0.46 & 0.63 & 0.53\end{array}$

$\begin{array}{lllllllllllllllll}0.93 & 0.93 & 0.92 & 0.92 & 0.93 & 0.89 & 0.92 & 0.90 & 0.87 & 0.80 & 0.88 & 0.78 & 0.45 & 0.63 & 0.53\end{array}$

$\begin{array}{llllllllllllllll}0.93 & 0.93 & 0.92 & 0.92 & 0.93 & 0.88 & 0.91 & 0.89 & 0.86 & 0.79 & 0.87 & 0.78 & 0.45 & 0.62 & 0.52\end{array}$

$\begin{array}{llllllllllllllll}0.93 & 0.93 & 0.92 & 0.92 & 0.92 & 0.88 & 0.91 & 0.89 & 0.86 & 0.79 & 0.87 & 0.78 & 0.44 & 0.62 & 0.52\end{array}$ 
Experiment

LEU-MISC-THERM-002-001 LEU-MISC-THERM-002-002 LEU-MISC-THERM-002-003 LEU-MISC-THERM-002-004 LEU-MISC-THERM-002-005 LEU-MISC-THERM-002-006 LEU-MISC-THERM-003-001_ LEU-MISC-THERM-003-002 LEU-MISC-THERM-003-003 LEU-MISC-THERM-003-004 LEU-MISC-THERM-003-005 LEU-MISC-THERM-003-006 LEU-MISC-THERM-003-007 LEU-MISC-THERM-003-008 LEU-MISC-THERM-003-009 LEU-MISC-THERM-003-010 LEU-MISC-THERM-003-011_ LEU-MISC-THERM-003-012 LEU-MISC-THERM-003-013 LEU-MISC-THERM-003-014 LEU-MISC-THERM-003-015 LEU-MISC-THERM-005-001_ LEU-MISC-THERM-005-002 LEU-MISC-THERM-005-003 LEU-MISC-THERM-005-004 LEU-MISC-THERM-005-005 LEU-MISC-THERM-005-006 LEU-MISC-THERM-005-007 LEU-MISC-THERM-005-008 LEU-MISC-THERM-005-009 LEU-MISC-THERM-005-010 LEU-MISC-THERM-005-011_ LEU-MISC-THERM-005-012 LEU-MISC-THERM-006-001_ LEU-MISC-THERM-006-002 LEU-MISC-THERM-006-003 LEU-MISC-THERM-006-004_ LEU-MISC-THERM-006-005 LEU-MISC-THERM-006-006 LEU-MISC-THERM-006-007 LEU-MISC-THERM-006-008 LEU-MISC-THERM-006-009_

\begin{tabular}{|c|c|c|c|c|c|c|c|c|c|c|c|c|c|c|}
\hline 1 & 2 & 3 & 4 & 5 & 6 & 7 & 8 & 9 & 10 & 11 & 12 & 13 & 14 & 15 \\
\hline 0.94 & 0.95 & 0.93 & 0.94 & 0.94 & 0.89 & 0.92 & 0.90 & 0.87 & 0.78 & 0.87 & 0.77 & 0.45 & 0.63 & 05 \\
\hline 0.94 & 0.95 & 0.93 & 0.93 & 0.94 & 0.89 & 0.92 & 0.90 & 0.87 & 0.78 & 0.87 & 0.77 & 0.44 & 0.63 & \\
\hline 0.93 & 0.94 & 0.93 & 0.93 & 0.93 & 0.89 & 0.92 & 0.90 & 0.87 & 0.79 & 0.87 & 0.77 & 0.45 & 0.62 & \\
\hline 0.93 & 0.94 & 0.92 & 0.93 & 0.93 & 0.88 & 0.91 & 0.89 & 0.87 & 0.79 & 0.87 & 0.77 & 0.44 & 0.62 & \\
\hline 0.93 & 0.93 & 0.92 & 0.93 & 0.93 & 0.88 & 0.91 & 0.89 & 0.86 & 0.78 & 0.87 & 0.77 & 0.44 & 0.62 & \\
\hline 0.93 & 0.93 & 0.92 & 0.92 & 0.93 & 0.88 & 0.91 & 0.89 & 0.86 & 0.78 & 0.87 & 0.77 & 0.44 & 0.61 & \\
\hline 0.98 & 0.97 & 0.97 & 0.95 & 0.94 & 0.90 & 0.93 & 0.93 & 0.87 & 0.80 & 0.90 & 0.80 & 0.49 & 0.68 & \\
\hline 0.98 & 0.97 & 0.97 & 0.95 & 0.94 & 0.90 & 0.93 & 0.93 & 0.87 & 0.80 & 0.90 & 0.79 & 0.48 & 0.68 & \\
\hline 0.98 & 0.97 & 0.97 & 0.95 & 0.95 & 0.90 & 0.93 & 0.93 & 0.87 & 0.80 & 0.90 & 0.79 & 0.48 & 0.68 & \\
\hline 0.98 & 0.97 & 0.97 & 0.95 & 0.95 & 0.90 & 0.93 & 0.93 & 0.87 & 0.80 & 0.90 & 0.79 & 0.48 & 0.68 & \\
\hline 0.98 & 0.98 & 0.97 & 0.96 & 0.95 & 0.89 & 0.93 & 0.93 & 0.87 & 0.80 & 0.89 & 0.79 & 47 & 0.68 & \\
\hline 0.98 & 0.98 & 0.97 & 0.96 & 0.95 & 0.89 & 0.93 & 0.93 & 0.87 & 0.79 & 0.89 & 0.79 & 0.47 & 0.67 & \\
\hline 0.98 & 0.98 & 0.97 & 0.96 & 0.95 & 0.89 & 0.93 & 0.93 & 0.87 & 0.79 & 0.89 & 0.78 & 0.46 & 0.67 & \\
\hline 0.98 & 0.98 & 0.97 & 0.96 & 0.95 & 0.89 & 0.93 & 0.92 & 0.87 & 0.78 & 0.89 & 0.78 & 0.45 & 0.67 & \\
\hline 0.98 & 0.96 & 0.97 & 0.95 & 0.94 & 0.91 & 0.93 & 0.93 & 0.87 & 0.83 & 0.91 & 0.82 & 0.50 & 0.69 & \\
\hline 0.98 & 0.96 & 0.97 & 0.95 & 0.94 & 0.91 & 0.93 & 0.93 & 0.87 & 0.82 & 91 & 0.81 & .50 & 0.69 & \\
\hline 0.98 & 0.97 & 0.97 & 0.95 & 0.94 & 0.91 & 0.93 & 0.93 & 0.87 & 0.82 & 0.91 & 0.81 & 0.49 & 0.69 & \\
\hline 0.98 & 0.97 & 0.97 & 0.95 & 0.94 & 0.90 & 0.93 & 0.93 & 0.87 & 0.82 & 0.91 & 0.81 & 0.49 & 0.68 & \\
\hline 0.98 & 0.97 & 0.97 & 0.96 & 0.95 & 0.90 & 0.93 & 0.93 & 0.87 & 0.81 & 0.90 & 0.80 & 0.48 & 0.68 & \\
\hline 0.98 & 8 & 0.97 & 0.96 & 0.95 & 0.90 & 0.93 & 0.93 & 0.87 & 0.80 & 0.89 & 0.79 & 0.47 & 0.67 & \\
\hline 0.98 & 0.98 & 0.97 & 0.96 & 0.95 & 0.89 & 0.93 & 0.93 & 0.87 & 0.79 & 0.89 & 0.78 & 0.46 & 0.67 & \\
\hline 0.98 & 0.96 & 0.97 & 0.95 & 0.94 & 0.91 & 0.93 & 0.93 & 0.87 & 0.83 & 0.91 & 0.82 & 0.51 & 0.69 & \\
\hline 0.98 & 0.96 & 0.97 & 0.94 & 0.93 & 0.91 & 0.93 & 0.93 & 0.87 & 0.83 & 0.91 & 0.82 & 0.52 & 0.70 & \\
\hline 0.98 & 0.95 & 0.97 & 0.94 & 0.93 & 0.91 & 0.93 & 0.93 & 0.86 & 0.84 & 0.92 & 0.83 & 0 . & 0.70 & \\
\hline 0.98 & 0.95 & 0.97 & 0.94 & 0.93 & 0.91 & 0.93 & 0.93 & 0.86 & 0.84 & 0.92 & 0.83 & 0.52 & 0.70 & \\
\hline 0.98 & 0.95 & 0.97 & 0.94 & 0.93 & 0.91 & 0.93 & 0.93 & 0.86 & 0.84 & 0.92 & 0.83 & 0.52 & 0.70 & \\
\hline 0.98 & 0.95 & 0.97 & 0.93 & 0.92 & 0.91 & 0.93 & 0.92 & 0.86 & 0.84 & 0.92 & 0.83 & 0.53 & 0.70 & \\
\hline 0.98 & 0.95 & 0.97 & 0.93 & 0.92 & 0.91 & 0.93 & 0.92 & 0.86 & 0.84 & 0.92 & 0.83 & 0.53 & 0.70 & \\
\hline 0.98 & 0.95 & 0.97 & 0.93 & 0.92 & 0.91 & 0.93 & 0.92 & 0.86 & 0.84 & 0.92 & 0.83 & 0. & 0.70 & \\
\hline 0.98 & 0.95 & 0.97 & 0.93 & 0.92 & 0.91 & 0.92 & 0.92 & 0.86 & 0.84 & 0.92 & 0.83 & 0.53 & 0.70 & \\
\hline 0.98 & 0.95 & 0.97 & 0.93 & 0.92 & 0.91 & 0.92 & 0.92 & 0.86 & 0.84 & 0.92 & 0.83 & 0.53 & 0.70 & \\
\hline 0.98 & 0.95 & 0.97 & 0.93 & 0.92 & 0.91 & 0.92 & 0.92 & 0.86 & 0.84 & 0.92 & 0.83 & 0.53 & 0.70 & \\
\hline 0.98 & 0.95 & 0.97 & 0.93 & 0.92 & 0.91 & 0.92 & 0.92 & 0.86 & 0.84 & 0.92 & 0.83 & 0.53 & 0.70 & \\
\hline 0.97 & 0.96 & 0.97 & 0.94 & 0.93 & 0.91 & 0.93 & 0.93 & 0.87 & 0.84 & 0.92 & 0.83 & 0.51 & 0.69 & \\
\hline 0.97 & 0.95 & 0.96 & 0.93 & 0.92 & 0.90 & 0.92 & 0.92 & 0.86 & 0.84 & 0.92 & 0.84 & 0.51 & 0.69 & \\
\hline 0.96 & 0.93 & 0.95 & 0.92 & 0.91 & 0.89 & 0.91 & 0.91 & 0.84 & 0.84 & 0.91 & 0.85 & 0.51 & 0.69 & \\
\hline 0.95 & 0.92 & 0.94 & 0.90 & 0.89 & 0.88 & 0.89 & 0.89 & 0.83 & 0.84 & 0.91 & 0.85 & 0.52 & 0.68 & \\
\hline 0.93 & 0.89 & 0.92 & 0.88 & 0.86 & 0.86 & 0.87 & 0.87 & 0.81 & 0.84 & 0.90 & 0.85 & 0.52 & 0.68 & \\
\hline 0.98 & 0.97 & 0.97 & 0.95 & 0.94 & 0.90 & 0.93 & 0.92 & 0.86 & 0.82 & 0.90 & 0.81 & 0.49 & 0.69 & \\
\hline 0.97 & 0.96 & 0.97 & 0.94 & 0.93 & 0.89 & 0.92 & 0.92 & 0.86 & 0.82 & 0.91 & 0.82 & 0.49 & 0.68 & \\
\hline 0.96 & 0.94 & 0.96 & 0.92 & 0.91 & 0.89 & 0.91 & 0.91 & 0.84 & 0.83 & 0.91 & 0.84 & 0.50 & 0.69 & \\
\hline 0.95 & 0.93 & 0.95 & 0.91 & 0.90 & 0.88 & 0.90 & 0.90 & 0.83 & 0.83 & 0.91 & 0.84 & 0.51 & 0.68 & \\
\hline
\end{tabular}


Experiment

LEU-MISC-THERM-006-010 LEU-SOL-THERM-001-001_E LEU-SOL-THERM-002-001 E LEU-SOL-THERM-002-002_E LEU-SOL-THERM-002-003_E LEU-SOL-THERM-003-001 E LEU-SOL-THERM-003-002_E LEU-SOL-THERM-003-003_E LEU-SOL-THERM-003-004 E LEU-SOL-THERM-003-005_E LEU-SOL-THERM-003-006 E LEU-SOL-THERM-003-007 E LEU-SOL-THERM-003-008_E LEU-SOL-THERM-003-009 E LEU-SOL-THERM-004-001 E LEU-SOL-THERM-004-002_E LEU-SOL-THERM-004-003 E LEU-SOL-THERM-004-004 E LEU-SOL-THERM-004-005_E LEU-SOL-THERM-004-006_E LEU-SOL-THERM-004-007 E LEU-SOL-THERM-005-001_E LEU-SOL-THERM-005-002_E LEU-SOL-THERM-005-003 E LEU-SOL-THERM-006-001_E LEU-SOL-THERM-006-002_E LEU-SOL-THERM-006-003 E LEU-SOL-THERM-006-004_E LEU-SOL-THERM-006-005_E LEU-SOL-THERM-007-001 E LEU-SOL-THERM-007-002_E LEU-SOL-THERM-007-003_E LEU-SOL-THERM-007-004 E LEU-SOL-THERM-007-005_E LEU-SOL-THERM-008-001_E LEU-SOL-THERM-008-002 E LEU-SOL-THERM-008-003_E LEU-SOL-THERM-008-004_E EU-SOL-THERM-009-001 E LEU-SOL-THERM-009-002_E LEU-SOL-THERM-009-003_E LEU-SOL-THERM-010-001_E

\begin{tabular}{|c|c|c|c|c|c|c|c|c|c|c|c|c|c|c|}
\hline 1 & 2 & 3 & 4 & 5 & 6 & 7 & 8 & 9 & 10 & 11 & 12 & 13 & 14 & 15 \\
\hline 0.94 & 0.91 & 0.93 & 0.89 & 0.88 & 0.86 & 0.88 & 0.88 & 0.82 & 0.83 & 0.90 & 0.84 & 0.51 & 0.68 & 0.57 \\
\hline 0.91 & 0.92 & 0.90 & 0.93 & 0.94 & 0.90 & 0.93 & 0.90 & 0.89 & 0.79 & 0.88 & 0.78 & 0.47 & 0.64 & 54 \\
\hline 0.80 & 0.76 & 0.79 & 0.77 & 0.78 & 0.81 & 0.80 & 0.77 & 0.75 & 0.77 & 0.81 & 0.75 & 0.46 & 0.55 & .43 \\
\hline 0.83 & 0.81 & 0.82 & 0.82 & 0.82 & 0.83 & 0.83 & 0.80 & 0.79 & 0.77 & 0.82 & 0.75 & 0.45 & 0.57 & .45 \\
\hline 0.82 & 0.79 & 0.81 & 0.80 & 0.81 & 0.83 & 0.82 & 0.79 & 0.78 & 0.77 & 0.82 & 0.75 & 0.45 & 0.56 & \\
\hline 0.82 & 0.84 & 0.81 & 0.84 & 0.86 & 0.82 & 0.85 & 0.81 & 0.82 & 0.71 & 0.79 & 0.71 & 0.38 & 0.52 & .44 \\
\hline 0.81 & 0.82 & 0.80 & 0.82 & 0.84 & 0.81 & 0.84 & 0.79 & 0.81 & 0.72 & 0.79 & 0.71 & 0.39 & 0.52 & .43 \\
\hline 0.81 & 0.81 & 0.80 & 0.82 & 0.84 & 0.81 & 0.83 & 0.79 & 0.80 & 0.73 & 0.79 & 0.72 & 0.39 & 0.52 & 0.43 \\
\hline 0.81 & 0.81 & 0.80 & 0.82 & 0.83 & 0.81 & 0.83 & 0.79 & 0.80 & 0.73 & 0.79 & 0.72 & 0.40 & 0.52 & .43 \\
\hline 0.76 & 0.73 & 0.76 & 0.74 & 0.75 & 0.78 & 0.77 & 0.74 & 0.74 & 0.74 & 0.78 & 0.73 & 0.42 & 0.51 & .40 \\
\hline 0.75 & 0.71 & 0.75 & 0.73 & 0.74 & 0.77 & 0.76 & 0.73 & 0.72 & 0.74 & 0.78 & 0.72 & 0.42 & 0.51 & 3 \\
\hline 0.75 & 0.70 & 0.74 & 0.72 & 0.72 & 0.77 & 0.75 & 0.72 & 0.71 & 0.74 & 0.77 & 0.72 & 0.42 & 0.50 & \\
\hline 0.69 & 0.61 & 0.68 & 0.64 & 0.64 & 0.72 & 0.68 & 0.66 & 0.64 & 0.74 & 0.74 & 0.71 & 0.43 & 0.48 & \\
\hline 0.69 & 0.61 & 0.68 & 0.63 & 0.63 & 0.72 & 0.67 & 0.65 & 0.63 & 0.73 & 0.73 & 1 & 0.43 & 0.48 & .3 \\
\hline 0.82 & 0.83 & 0.82 & 0.84 & 0.86 & 0.83 & 0.86 & 0.82 & 0.83 & 0.74 & 0.81 & .73 & 0.40 & 0.54 & .44 \\
\hline 0.81 & 0.82 & 0.81 & 0.83 & 0.85 & 0.83 & 0.85 & 0.81 & 0.82 & 0.74 & 0.81 & 0.73 & 0.41 & 0.54 & 0.4 \\
\hline 0.81 & 0.80 & 0.80 & 0.82 & 0.83 & 0.82 & 0.84 & 0.80 & 0.81 & 0.75 & 0.81 & 0.73 & 0.41 & 0.53 & 0.43 \\
\hline 0.80 & 0.79 & 0.79 & 0.80 & 0.81 & 0.82 & 0.82 & 0.79 & 0.80 & 0.75 & 0.80 & 0.74 & 0.42 & 0.53 & .42 \\
\hline 0.79 & 0.77 & 0.78 & 0.79 & 0.80 & 0.81 & 0.81 & 0.78 & 0.78 & 0.75 & 0.80 & .74 & 0.42 & 0.53 & .42 \\
\hline 0.78 & 0.76 & 0.78 & 0.77 & 0.78 & 0.80 & 0.80 & 0.77 & 0.77 & 0.75 & 0.80 & 0.74 & 0.42 & 0.53 & 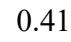 \\
\hline 0.77 & 0.74 & 0.77 & 0.76 & 0.77 & 0.80 & 0.79 & 0.76 & 0.76 & 0.76 & 0.79 & 0.74 & 0.43 & 0.52 & 0. \\
\hline 0.78 & 0.71 & 0.77 & 0.73 & 0.73 & 0.80 & 0.77 & 0.75 & 0.73 & 0.79 & 0.80 & 0.76 & 0.48 & 0.55 & 0.4 \\
\hline 0.77 & 0.70 & 0.76 & 0.72 & 0.72 & 0.80 & 0.76 & 0.74 & 0.72 & 0.79 & 0.80 & 0.77 & 0.48 & 0.55 & 0.4 \\
\hline 0.75 & 0.66 & 0.74 & 0.68 & 0.67 & 0.78 & 0.73 & 0.71 & 0.68 & 0.79 & 0.79 & 0.77 & 0.50 & 0.55 & 0.3 \\
\hline 0.84 & 0.87 & 0.84 & 0.89 & 0.91 & 0.86 & 0.90 & 0.86 & 0.88 & 0.75 & 0.83 & 0.74 & 0.41 & 0.56 & 0.4 \\
\hline 0.85 & 0.86 & 0.84 & 0.88 & 0.90 & 0.86 & 0.90 & 0.86 & 0.87 & 0.76 & .84 & 0.75 & 0.42 & 0.56 & 0.4 \\
\hline 0.85 & 0.86 & 0.85 & 0.87 & 0.89 & 0.87 & 0.89 & 0.86 & 0.87 & 0.77 & 0.84 & 0.77 & 0.43 & 0.57 & 0.4 \\
\hline 0.85 & 0.86 & 0.85 & 0.87 & 0.89 & 0.87 & 0.89 & 0.86 & 0.87 & 0.78 & 0.85 & 0.77 & 0.44 & 0.57 & 04 \\
\hline 0.85 & 0.84 & 0.85 & 0.85 & 0.87 & 0.87 & 0.88 & 0.85 & 0.85 & 0.80 & 0.86 & 0.79 & 0.46 & 0.58 & .47 \\
\hline 0.82 & 0.84 & 0.82 & 0.85 & 0.87 & 0.82 & 0.85 & 0.81 & 0.83 & 0.72 & & 0.71 & 0.39 & 0.53 & 0.4 \\
\hline 0.81 & 0.83 & 0.81 & 0.83 & 0.85 & 0.82 & 0.84 & 0.80 & 0.82 & 0.72 & 0.80 & 0.71 & 0.39 & 0.53 & 0.4 \\
\hline 0.81 & 0.81 & 0.80 & & 0.84 & 0.81 & 0.83 & 0.79 & 0.80 & 0.73 & 0.80 & 0.72 & 0.40 & 0.53 & 0.4 \\
\hline 0.80 & 0.80 & 0.80 & 0.81 & 0.82 & 0.81 & 0.82 & 0.78 & 0.79 & 0.74 & 0.80 & 0.72 & 0.40 & 0.52 & 0.42 \\
\hline 0.79 & 0.78 & 0.79 & 0.79 & 0.81 & 0.80 & 0.81 & 0.77 & 0.78 & 0.74 & 0.79 & 0.73 & 0.41 & 0.52 & 0.42 \\
\hline 0.79 & 0.77 & 0.79 & 0.78 & 0.80 & 0.81 & 0.81 & 0.77 & 0.78 & 0.75 & 0.80 & 0.74 & 0.42 & 0.53 & 0.42 \\
\hline 0.79 & 0.77 & 0.78 & 0.78 & 0.79 & 0.81 & 0.81 & 0.77 & 0.78 & 0.76 & 0.80 & 0.74 & 0.42 & 0.53 & 0.4 \\
\hline 0.79 & 0.77 & 0.78 & 0.78 & 0.79 & 0.81 & 0.81 & 0.78 & 0.78 & 0.76 & 0.80 & 0.74 & 0.43 & 0.53 & 0.4 \\
\hline 0.79 & 0.76 & 0.78 & 0.78 & 0.79 & 0.81 & 0.81 & 0.77 & 0.78 & 0.76 & 0.80 & 0.74 & 0.43 & 0.53 & 0.4 \\
\hline 0.79 & 0.77 & 0.79 & 0.78 & 0.80 & 0.81 & 0.81 & 0.77 & 0.78 & 0.75 & 0.80 & 0.74 & 0.42 & 0.53 & 0.42 \\
\hline 0.79 & 0.77 & 0.79 & 0.79 & 0.80 & 0.81 & 0.81 & 0.77 & 0.78 & 0.75 & 0.80 & 0.73 & 0.42 & 0.53 & 0.4 \\
\hline 0.79 & 0.77 & 0.79 & 0.79 & 0.80 & 0.81 & 0.81 & 0.77 & 0.78 & 0.75 & 0.80 & 0.73 & 0.42 & 0.53 & 0.4 \\
\hline 0.79 & 0.77 & 0.79 & 0.79 & 0.80 & 0.81 & 0.81 & 0.78 & 0.78 & 0.75 & 0.80 & 0.73 & 0.42 & 0.53 & \\
\hline
\end{tabular}
.54

0.43 0.44 .39 0.44 . 47 47 0.43 0.42

0.44 43 .43 .39 .35 44 .42 42 41 41 47 .47 44 .42 .42 0.42 42 0.42
0.42 
Experiment

LEU-SOL-THERM-010-002_E LEU-SOL-THERM-010-003_E LEU-SOL-THERM-010-004 E LEU-SOL-THERM-011-001_E LEU-SOL-THERM-011-002_E LEU-SOL-THERM-011-003 E LEU-SOL-THERM-011-004 E LEU-SOL-THERM-011-005_E LEU-SOL-THERM-011-006 E LEU-SOL-THERM-011-007_E LEU-SOL-THERM-011-008 E LEU-SOL-THERM-011-009 E LEU-SOL-THERM-011-010_E LEU-SOL-THERM-011-011 E LEU-SOL-THERM-011-012 E LEU-SOL-THERM-011-013_E LEU-SOL-THERM-016-001_E LEU-SOL-THERM-016-002 E LEU-SOL-THERM-016-003_E LEU-SOL-THERM-016-004_E LEU-SOL-THERM-016-005 E LEU-SOL-THERM-016-006_E LEU-SOL-THERM-016-007_E LEU-SOL-THERM-017-001 E LEU-SOL-THERM-017-002_E LEU-SOL-THERM-017-003_E LEU-SOL-THERM-017-004 E LEU-SOL-THERM-017-005_E LEU-SOL-THERM-017-006_E LEU-SOL-THERM-018-001 E LEU-SOL-THERM-018-002_E LEU-SOL-THERM-018-003_E LEU-SOL-THERM-018-004 E LEU-SOL-THERM-018-005_E LEU-SOL-THERM-018-006_E LEU-SOL-THERM-019-001 E LEU-SOL-THERM-019-002_E LEU-SOL-THERM-019-003 E LEU-SOL-THERM-019-004 LEU-SOL-THERM-019-005_E LEU-SOL-THERM-019-006_E LEU-SOL-THERM-020-001_E

\begin{tabular}{|c|c|c|c|c|c|c|c|c|c|c|c|c|c|c|}
\hline 1 & 2 & 3 & 4 & 5 & 6 & 7 & 8 & 9 & 10 & 11 & 12 & 13 & 14 & 5 \\
\hline 0.79 & 0.77 & 0.78 & 0.79 & 0.80 & 0.81 & 0.81 & 0.78 & 0.78 & 0.75 & 0.80 & 0.74 & 0.42 & 0.53 & (4) \\
\hline 0.79 & 0.77 & 0.78 & 0.79 & 0.80 & 0.81 & 0.81 & 0.78 & 0.78 & 0.75 & 0.80 & 0.74 & 0.42 & 0.53 & \\
\hline 0.79 & 0.77 & 0.78 & 0.79 & 0.80 & 0.81 & 0.81 & 0.78 & 0.78 & 0.75 & 0.80 & 0.74 & 0.42 & 0.53 & \\
\hline 0.83 & 0.80 & 0.82 & 0.82 & 0.82 & 0.84 & 0.84 & 0.81 & 0.80 & 0.79 & 0.83 & 0.77 & 0.46 & 0.57 & \\
\hline 0.82 & 0.79 & 0.81 & 0.80 & 0.81 & 0.84 & 0.83 & 0.80 & 0.79 & 0.79 & 0.83 & 0.77 & 0.46 & 0.57 & \\
\hline 0.81 & 0.77 & 0.80 & 0.78 & 0.79 & 0.83 & 0.81 & 0.78 & 0.77 & 0.79 & 0.82 & 0.77 & 0.47 & 0.56 & \\
\hline 0.79 & 0.74 & 0.78 & 0.76 & 0.77 & 0.81 & 0.80 & 0.77 & 0.76 & 0.79 & 0.81 & 0.76 & 0.47 & 0.55 & \\
\hline 0.78 & 0.73 & 0.77 & 0.75 & 0.75 & 0.81 & 0.78 & 0.75 & 0.74 & 0.78 & 0.81 & 0.76 & 0.47 & 0.55 & \\
\hline 0.77 & 0.71 & 0.76 & 0.73 & 0.73 & 0.79 & 0.76 & 0.74 & 0.72 & 0.78 & 0.80 & 0.76 & 0.47 & 0.54 & \\
\hline 0.76 & 0.69 & 0.75 & 0.71 & 0.71 & 0.79 & 0.75 & 0.73 & 0.71 & 0.78 & 0.79 & 0.76 & 0.47 & 0.54 & \\
\hline 0.83 & 0.81 & 0.83 & 0.82 & 0.83 & 0.84 & 0.84 & 0.81 & 0.80 & 0.77 & 0.83 & 0.76 & 0.45 & 0.57 & \\
\hline 0.83 & 0.80 & 0.82 & 0.81 & 0.82 & 0.83 & 0.83 & 0.80 & 0.79 & 0.78 & 0.82 & 0.76 & 0.45 & 0.56 & \\
\hline 0.81 & 0.78 & 0.80 & 0.79 & 0.80 & 0.82 & 0.82 & 0.79 & 0.77 & 0.78 & 0.82 & 0.76 & 0.46 & 0.56 & \\
\hline 0.80 & 75 & 0.79 & 0.77 & 0.77 & 0.81 & 0.80 & 0.77 & 0.75 & 0.78 & 0.81 & 0.76 & 0.46 & 0.55 & \\
\hline 0.79 & 0.74 & 0.78 & 0.75 & 0.75 & 0.80 & 0.78 & 0.76 & 0.74 & 0.78 & 0.81 & 0.76 & 0.46 & 0.55 & \\
\hline 0.77 & 0.71 & 0.77 & 0.73 & 0.73 & 0.79 & 0.77 & 0.74 & 0.72 & 0.78 & 0.80 & 0.75 & 0.46 & 0.54 & \\
\hline 0.84 & 0.88 & 0.84 & 0.90 & 0.92 & 0.86 & 0.90 & 0.86 & 0.88 & 0.74 & 0.83 & 0.73 & 0.40 & 0.56 & \\
\hline 0.84 & 0.87 & 0.84 & 0.89 & 0.91 & 0.86 & 0.90 & 0.86 & 0.88 & 0.74 & 0.83 & 0.74 & 0.40 & 0.56 & \\
\hline 0.83 & 0.85 & 0.83 & 0.88 & 0.89 & 0.86 & 0.89 & 0.85 & 0.87 & 0.75 & 0.83 & 0.74 & 0.41 & 0.55 & \\
\hline 0.83 & 0.85 & 0.83 & 0.87 & 0.89 & 0.85 & 0.88 & 0.84 & 0.86 & 0.76 & 0.83 & 0.75 & 0.42 & 0.55 & \\
\hline 0.83 & 0.84 & 0.82 & 0.86 & 0.88 & 0.85 & 0.88 & 0.84 & 0.86 & 0.76 & 0.83 & 0.75 & 0.42 & 0.55 & \\
\hline 0.82 & 0.83 & 0.82 & 0.85 & 0.87 & 0.85 & 0.87 & 0.83 & 0.85 & 0.76 & 0.83 & 0.75 & 0.42 & 0.55 & \\
\hline 0.82 & 0.82 & 0.81 & 0.84 & 0.86 & 0.85 & 0.87 & 0.83 & 0.84 & 0.77 & 0.83 & 0.76 & 0.43 & 0.55 & \\
\hline 0.84 & 0.88 & 0.84 & 0.89 & 0.91 & 0.85 & 0.89 & 0.85 & 0.87 & 0.73 & .82 & 0.72 & 0.39 & 0.55 & \\
\hline 0.84 & 0.87 & 0.83 & 0.89 & 0.91 & 0.84 & 0.89 & 0.85 & 0.87 & 0.72 & 0.81 & 0.72 & 0.39 & 0.55 & \\
\hline 0.83 & 0.86 & 0.83 & 0.87 & 0.89 & 0.84 & 0.88 & 0.84 & 0.86 & 0.73 & 0.81 & 0.73 & 0.39 & 0.54 & \\
\hline 0.83 & 0.85 & 0.82 & 0.86 & 0.88 & 0.84 & 0.88 & 0.83 & 0.85 & 0.74 & 0.82 & 0.73 & 0.40 & 0.54 & \\
\hline 0.82 & 0.84 & 0.82 & 0.86 & 0.87 & 0.84 & 0.87 & 0.83 & 0.84 & 0.75 & 0.82 & 0.74 & 0.41 & 0.54 & \\
\hline 0.82 & 0.83 & 0.82 & 0.85 & 0.87 & 0.84 & 0.87 & 0.83 & 0.84 & 0.75 & 0.82 & 0.74 & 0.41 & 0.54 & \\
\hline 0.82 & 0.82 & 0.82 & 0.84 & 0.86 & 0.85 & 0.87 & 0.83 & 0.84 & 0.77 & 0.83 & 0.76 & 0.43 & 0.55 & \\
\hline 0.82 & 0.83 & 0.82 & 0.85 & 0.86 & 0.84 & 0.86 & 0.82 & 0.84 & 0.76 & 0.82 & 0.75 & 0.42 & 0.55 & \\
\hline 0.82 & 0.83 & 0.82 & 0.85 & 0.86 & 0.84 & 0.87 & 0.83 & 0.84 & 0.76 & 0.83 & 0.75 & 0.42 & 0.55 & \\
\hline 0.82 & 0.83 & 0.82 & 0.85 & 0.86 & 0.85 & 0.87 & 0.83 & 0.84 & 0.76 & 0.83 & 0.75 & 0.42 & 0.55 & \\
\hline 0.82 & 0.83 & 0.82 & 0.85 & 0.86 & 0.85 & 0.87 & 0.83 & 0.84 & 0.77 & 0.83 & 0.76 & 0.43 & 0.55 & \\
\hline 0.82 & 0.82 & 0.82 & 0.85 & 0.86 & 0.85 & 0.87 & 0.83 & 0.84 & 0.77 & 0.83 & 0.76 & 0.43 & 0.55 & \\
\hline 0.82 & 0.83 & 0.82 & 0.85 & 0.87 & 0.84 & 0.87 & 0.83 & 0.84 & 0.75 & 0.82 & 0.74 & 0.41 & 0.55 & \\
\hline 0.82 & 0.83 & 0.82 & 0.85 & 0.87 & 0.85 & 0.87 & 0.83 & 0.84 & 0.76 & 0.83 & 0.75 & 0.42 & 0.55 & \\
\hline 0.82 & 0.83 & 0.82 & 0.85 & 0.87 & 0.85 & 0.87 & 0.83 & 0.85 & 0.76 & 0.83 & 0.75 & 0.42 & 0.55 & \\
\hline 0.82 & 0.83 & 0.82 & 0.85 & 0.87 & 0.85 & 0.87 & 0.83 & 0.85 & 0.76 & 0.83 & 0.75 & 0.42 & 0.55 & \\
\hline 0.82 & 0.83 & 0.82 & 0.85 & 0.87 & 0.85 & 0.87 & 0.83 & 0.85 & 0.76 & 0.83 & 0.75 & 0.42 & 0.55 & \\
\hline 0.82 & 0.83 & 0.82 & 0.85 & 0.87 & 0.85 & 0.87 & 0.83 & 0.85 & 0.76 & 0.83 & 0.75 & 0.42 & 0.55 & \\
\hline 0.80 & 0.78 & 0.79 & 0.80 & 0.81 & 0.82 & 0.82 & 0.79 & 0.79 & 0.75 & 0.81 & 0.74 & 0.42 & 0.53 & \\
\hline
\end{tabular}
.42 0.42 41 44 42 47 0.45 .47 0.45 44 0.44 45 5 45 .45 0.43 .42 45 .42 47 46 44 46
45 .45 45
44 45 0.45 45
.45 42 
Case

1555

1556

1557

1558

1559

1560

1561

1562

1563

1564

1565

1566

1567

1568

1569

1570

1571

1572

1573

1574

1575

1576

1577

1578

1579

1580

1581

1582

1583

1584
Experiment

LEU-SOL-THERM-020-002_E LEU-SOL-THERM-020-003_E

LEU-SOL-THERM-020-004 E LEU-SOL-THERM-022-001_E LEU-SOL-THERM-022-002_E EU-SOL-THERM-022-003 LEU-SOL-THERM-022-004_E LEU-SOL-THERM-023-001_E EU-SOL-THERM-023-002 E LEU-SOL-THERM-023-003_E LEU-SOL-THERM-023-004_E LEU-SOL-THERM-023-005 E LEU-SOL-THERM-023-006_E LEU-SOL-THERM-023-007_E LEU-SOL-THERM-023-008 E LEU-SOL-THERM-023-009_E LEU-SOL-THERM-024-001_E LEU-SOL-THERM-024-002 E LEU-SOL-THERM-024-003_E LEU-SOL-THERM-024-004_E LEU-SOL-THERM-024-005 E LEU-SOL-THERM-024-006_E LEU-SOL-THERM-024-007_E LEU-SOL-THERM-025-001 E LEU-SOL-THERM-025-002_E LEU-SOL-THERM-025-003_E LEU-SOL-THERM-025-004 E LEU-SOL-THERM-025-005_E LEU-SOL-THERM-025-006_E LEU-SOL-THERM-025-007_E $\begin{array}{ccccc}\mathbf{1} & \mathbf{2} & \mathbf{3} & \mathbf{4} & \mathbf{5}\end{array}$

$\begin{array}{llll}0.78 & 0.75 & 0.78 & 0.77 \\ 0.76 & 0.72 & 0.75 & 0.74\end{array}$

$\begin{array}{llll}0.82 & 0.82 & 0.82 & 0.84\end{array}$

$\begin{array}{lllllllll}0.82 & 0.82 & 0.82 & 0.84 & 0.86 & 0.84 & 0.86 & 0.82 & 0.83\end{array}$

$\begin{array}{lllllllll}0.82 & 0.80 & 0.81 & 0.83 & 0.84 & 0.85 & 0.86 & 0.82 & 0.83\end{array}$

$\begin{array}{llll}0.82 & 0.81 & 0.82 & 0.83\end{array}$

$\begin{array}{llll}0.82 & 0.81 & 0.82 & 0.83\end{array}$

$\begin{array}{llll}0.82 & 0.81 & 0.81 & 0.83\end{array}$

$\begin{array}{lllll}0.82 & 0.81 & 0.81 & 0.83\end{array}$

$\begin{array}{llll}0.82 & 0.81 & 0.81 & 0.83\end{array}$

$0.82 \quad 0.82$

$\begin{array}{ll}0.82 & 0.82\end{array}$

$\begin{array}{lll}0.82 & 0.81 & 0.82\end{array}$

$\begin{array}{llll}0.82 & 0.81 & 0.82 & 0.83 \\ 0.82 & 0.81 & 0.82 & 0.83\end{array}$

$\begin{array}{llll}0.82 & 0.81 & 0.82 & 0.83\end{array}$

$\begin{array}{llll}0.82 & 0.82 & 0.82 & 0.84\end{array}$

$0.82 \quad 0.82$

$\begin{array}{llll}0.82 & 0.82 & 0.82 & 0.84\end{array}$

$\begin{array}{llll}0.82 & 0.82 & 0.82 & 0.84\end{array}$

$\begin{array}{llll}0.82 & 0.80 & 0.81 & 0.83\end{array}$

$\begin{array}{llll}0.82 & 0.81 & 0.81 & 0.83 \\ 0.82 & 0.81 & 0.81 & 0.83\end{array}$

$\begin{array}{llll}0.82 & 0.81 & 0.81 & 0.83\end{array}$

$\begin{array}{llll}0.82 & 0.81 & 0.81 & 0.83\end{array}$

$\begin{array}{llll}0.82 & 0.81 & 0.81 & 0.83\end{array}$

$\begin{array}{llll}0.82 & 0.82 & 0.82 & 0.83\end{array}$

$\begin{array}{llll}0.82 & 0.82 & 0.82 & 0.83\end{array}$ $\begin{array}{lllll}0.86 & 0.84 & 0.86 & 0.82 & 0.83\end{array}$

$\begin{array}{cccccccc}\mathbf{5} & \mathbf{6} & \mathbf{7} & \mathbf{8} & \mathbf{9} & \mathbf{1 0} & \mathbf{1 1} & \mathbf{1 2} \\ 0.79 & 0.80 & 0.80 & 0.77 & 0.77 & 0.76 & 0.80 & 0.74 \\ 0.75 & 0.78 & 0.77 & 0.74 & 0.74 & 0.75 & 0.78 & 0.73 \\ 0.71 & 0.77 & 0.74 & 0.72 & 0.71 & 0.75 & 0.77 & 0.73 \\ 0.86 & 0.84 & 0.86 & 0.82 & 0.84 & 0.76 & 0.83 & 0.75 \\ 0.86 & 0.84 & 0.86 & 0.82 & 0.83 & 0.76 & 0.83 & 0.75 \\ 0.86 & 0.84 & 0.86 & 0.82 & 0.83 & 0.76 & 0.83 & 0.75 \\ 0.86 & 0.84 & 0.86 & 0.82 & 0.83 & 0.76 & 0.83 & 0.75 \\ 0.84 & 0.85 & 0.86 & 0.82 & 0.83 & 0.79 & 0.84 & 0.77 \\ 0.85 & 0.85 & 0.86 & 0.82 & 0.83 & 0.78 & 0.84 & 0.77 \\ 0.85 & 0.84 & 0.86 & 0.82 & 0.83 & 0.78 & 0.83 & 0.76 \\ 0.84 & 0.84 & 0.85 & 0.82 & 0.83 & 0.78 & 0.83 & 0.76 \\ 0.84 & 0.84 & 0.85 & 0.81 & 0.82 & 0.77 & 0.83 & 0.76 \\ 0.85 & 0.83 & 0.85 & 0.81 & 0.82 & 0.76 & 0.82 & 0.75 \\ 0.85 & 0.83 & 0.85 & 0.81 & 0.82 & 0.76 & 0.82 & 0.75 \\ 0.85 & 0.83 & 0.85 & 0.81 & 0.82 & 0.76 & 0.82 & 0.75 \\ 0.85 & 0.83 & 0.85 & 0.81 & 0.82 & 0.75 & 0.82 & 0.74 \\ 0.84 & 0.85 & 0.86 & 0.82 & 0.83 & 0.79 & 0.84 & 0.77 \\ 0.84 & 0.85 & 0.86 & 0.82 & 0.83 & 0.78 & 0.84 & 0.77 \\ 0.85 & 0.84 & 0.86 & 0.82 & 0.83 & 0.77 & 0.83 & 0.76 \\ 0.85 & 0.84 & 0.86 & 0.82 & 0.83 & 0.76 & 0.83 & 0.75 \\ 0.85 & 0.84 & 0.86 & 0.82 & 0.83 & 0.76 & 0.82 & 0.75 \\ 0.85 & 0.84 & 0.86 & 0.82 & 0.83 & 0.76 & 0.83 & 0.75 \\ 0.85 & 0.84 & 0.86 & 0.82 & 0.83 & 0.76 & 0.82 & 0.75 \\ 0.84 & 0.85 & 0.85 & 0.82 & 0.83 & 0.78 & 0.84 & 0.77 \\ 0.84 & 0.84 & 0.85 & 0.82 & 0.83 & 0.78 & 0.83 & 0.77 \\ 0.84 & 0.84 & 0.85 & 0.82 & 0.83 & 0.78 & 0.83 & 0.76 \\ 0.85 & 0.84 & 0.85 & 0.82 & 0.83 & 0.77 & 0.83 & 0.76 \\ 0.85 & 0.84 & 0.85 & 0.81 & 0.83 & 0.77 & 0.83 & 0.75 \\ 0.85 & 0.84 & 0.85 & 0.82 & 0.83 & 0.77 & 0.83 & 0.75 \\ 0.85 & 0.84 & 0.85 & 0.82 & 0.83 & 0.76 & 0.83 & 0.75\end{array}$

15

0.41

0.40

0.38

0.44

0.44

0.44

0.44

0.44

0.44

0.44

0.44

0.44

0.44

0.44

0.44

0.44

0.44

0.44

0.44

0.44

0.44

0.44

0.44

0.44

0.44

0.44

0.44

0.44

0.44

0.44 


\section{APPENDIX B. TN-B1 WATER MODELING EFFECT ON BENCHMARK EXPERIMENT $c_{k}$}

The 6.7 wt. $\%{ }^{235} \mathrm{U}, 13 \mathrm{Gd}$ rods/assembly, $6 \times 1 \times 6 \mathrm{HAC}$ array model was assessed by using two different modeling approaches for water in the package. The intent of this study was to determine whether subdividing the different water regions by using different material numbers rather than by using one material number for multiple regions has an impact on $c_{k}$ and the identification of applicable experiments.

The first approach is documented in Section 5.4, which uses material 7 for water in the entire fuel lattice (Gd and non-Gd rods), in the space between poly blocks and fuel, and in the reflector. The second approach uses different material numbers for Gd rod lattice water, non-Gd rod lattice water, water in spaces, and the water reflector. Both cases have essentially the same forward and adjoint $k_{\text {eff }}$ and forward EALF within the uncertainty of the cases.

Base case: T3D_HAC_6-7wt_13Gd_6x6, 658 experiments with $c_{k} \geq 0.9$

Alternate case: T3D_HAC_6-7wt_13Gd_6x6_Hrefl, 646 experiments with $c_{k} \geq 0.9$

There is a shift of 12 benchmarks identified as "similar." In each case, the change in $c_{k}$ is small and involves a case with $c_{k}$ very close to 0.9 . The statistics in Table B-1 characterize the change in $c_{k}$ of the 1,584 benchmarks. The change is very small.

Table B-1. Statistics that characterize the change in $c_{k}$ of the 1,584 benchmarks (6 11 x 6 array).

\begin{tabular}{|c|c|}
\hline Statistic & $\begin{array}{c}\text { Change } \\
\text { in } \boldsymbol{c}_{\boldsymbol{k}}\end{array}$ \\
\hline Median base $c_{k}$ & 0.8902 \\
\hline Max diff. & 0.0014 \\
\hline Median diff. & 0.0004 \\
\hline Avg. diff. & 0.0003 \\
\hline Std. dev. diff. & 0.0003 \\
\hline
\end{tabular}

The same comparison process was used with the $8 \mathrm{wt} . \%{ }^{235} \mathrm{U}, 24 \mathrm{Gd}$ rods/assembly, $10 \times 1 \times 10 \mathrm{HAC}$ array model. As with the $6.7 \mathrm{wt} . \%$ model, both cases have essentially the same forward and adjoint $k_{\text {eff }}$ and forward EALF within the uncertainty of the cases.

Base case: T3D_HAC_8wt_24Gd_10x10a, 132 experiments with $c_{k} \geq 0.9$

Alternate case: T3D_HAC_8wt_24Gd_10x10_Hrefl, 126 experiments with $c_{k} \geq 0.9$

There is a shift of nine benchmarks identified as "similar." In each case, the change in $c_{k}$ is smallalthough larger than the $6.7 \mathrm{wt} . \%$ changes - and involves a case with $c_{k}$ very close to 0.9 . The statistics below characterize the change in ck of the 1584 benchmarks. The change is small, confirming that the details of water modeling in the TN-B1 are relatively unimportant to the desired result. 
Table B-2. Statistics that characterize the change in $c_{k}$ of the 1,584 benchmarks $(10 \times 1 \times 10$ array).

\begin{tabular}{|c|c|}
\hline Statistic & $\begin{array}{c}\text { Change in } \\
\boldsymbol{c}_{\boldsymbol{k}}\end{array}$ \\
\hline Median base $c_{k}$ & 0.8107 \\
\hline Max diff. & 0.0028 \\
\hline Median diff. & 0.0012 \\
\hline Avg. diff. & 0.0010 \\
\hline Std. dev. diff. & 0.0010 \\
\hline
\end{tabular}




\section{APPENDIX C. TN-B1 ARRAY SIZE MODELING EFFECT ON BENCHMARK EXPERIMENT $c_{k}$}

The $5 \mathrm{wt} . \%{ }^{235} \mathrm{U}, 13 \mathrm{Gd}$ rods/assembly, $10 \times 1 \times 10 \mathrm{HAC}$ array model was assessed by using two additional modeling approaches for TSUNAMI. The intent of this study was to determine whether using a single package - either with reflective boundary conditions or with a $30 \mathrm{~cm}$ water reflector-changes the identification of applicable benchmark experiments by using $c_{k}$.

At first glance, these systems might appear similar. The same enrichment, same fuel form, and some moderators are in use in all three systems. The physical size of the systems differ, and these differences drive differences in reactivity, neutron spectrum, and some sensitivities. Several sensitivities are provided in Table C-1, as well as the system $k_{\text {eff }}$ and the EALF value. SDF file names are provided in Table C-2. The large differences in $k_{\text {eff }}$ reflect the differences in physical size and the leakage of neutrons from the system. Similarly, the spectrum of neutrons in the system softens as the system gets larger neutrons are resident in the system for longer. This longer lifetime allows more scattering and greater thermalization of some of the neutrons.

Table C-1. Key Sensitivities Among the Three Models Considered

5 wt.\% TN-B1 sensitivities

\begin{tabular}{|c|c|c|c|}
\hline Nuclide & $\begin{array}{c}\text { Single } \\
\text { package }\end{array}$ & $\mathbf{1 0 0}$ array & $\begin{array}{c}\text { Inf. } \\
\text { array }\end{array}$ \\
\hline${ }^{1} \mathrm{H}\left(\mathrm{H}_{2} \mathrm{O}\right)$ & 0.391 & 0.109 & 0.03 \\
\hline${ }^{235} \mathrm{U}$ & 0.215 & 0.221 & 0.217 \\
\hline${ }^{1} \mathrm{H}($ poly $)$ & 0.148 & 0.054 & 0.033 \\
\hline${ }^{16} \mathrm{O}$ & 0.046 & 0.015 & 0.004 \\
\hline${ }^{238} \mathrm{U}$ & -0.017 & -0.073 & -0.085 \\
\hline$k_{\text {eff }}$ & 0.627 & 0.938 & 1.045 \\
\hline EALF & 0.333 & 0.248 & 0.233 \\
\hline
\end{tabular}

Table C-2. SDF File Names

\begin{tabular}{|l|}
\hline Single package \\
\hline 100 array \\
\hline Inf. array \\
\hline
\end{tabular}

T3D_HAC_5wt_13Gd_sgl.sdf
T3D_HAC_5wt_13Gd_10x10.sdf
T3D HAC 5wt 13Gd inf.sdf

The integral total ${ }^{235} \mathrm{U}$ sensitivity is largely invariant to system size in this scenario, although other sensitivities change significantly. The ${ }^{235} \mathrm{U}$ total sensitivity is shown in Figure C-1, which shows greater sensitivity at high energies and lower sensitivity at thermal energies for the single package model. This is consistent with the higher EALF value reported in Table C-1. 


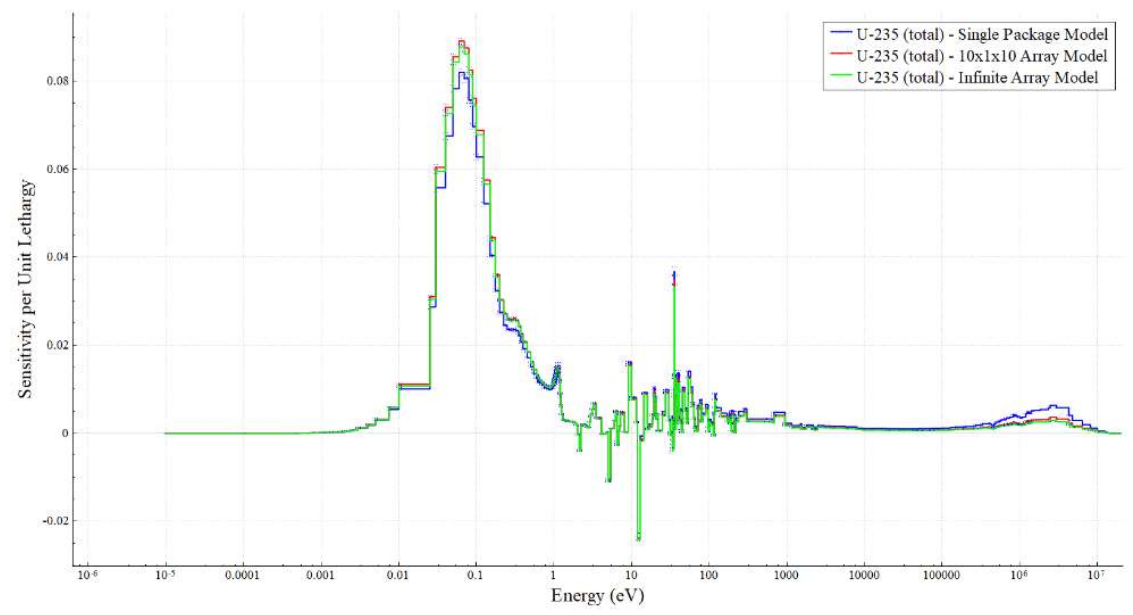

Figure C-1. Total ${ }^{235} \mathrm{U}$ sensitivity profiles for the three models.

All three moderating species $-{ }^{1} \mathrm{H}\left(\mathrm{H}_{2} \mathrm{O}\right),{ }^{1} \mathrm{H}($ poly $)$, and ${ }^{16} \mathrm{O}$ - have significantly higher sensitivity in the single package model. The profiles are shown in Figure C-2. The model is fairly small, and many neutrons will leak from the system if they are not scattered first. Hence, collisions with light nuclei that lead to large energy losses on average are very important in the small system. In the larger models, these nuclei are less important because the neutron has a much lower, or zero, leakage probability. Eventually, the neutrons will reach thermal energy, although slowing down also increases the probability of parasitic absorption.

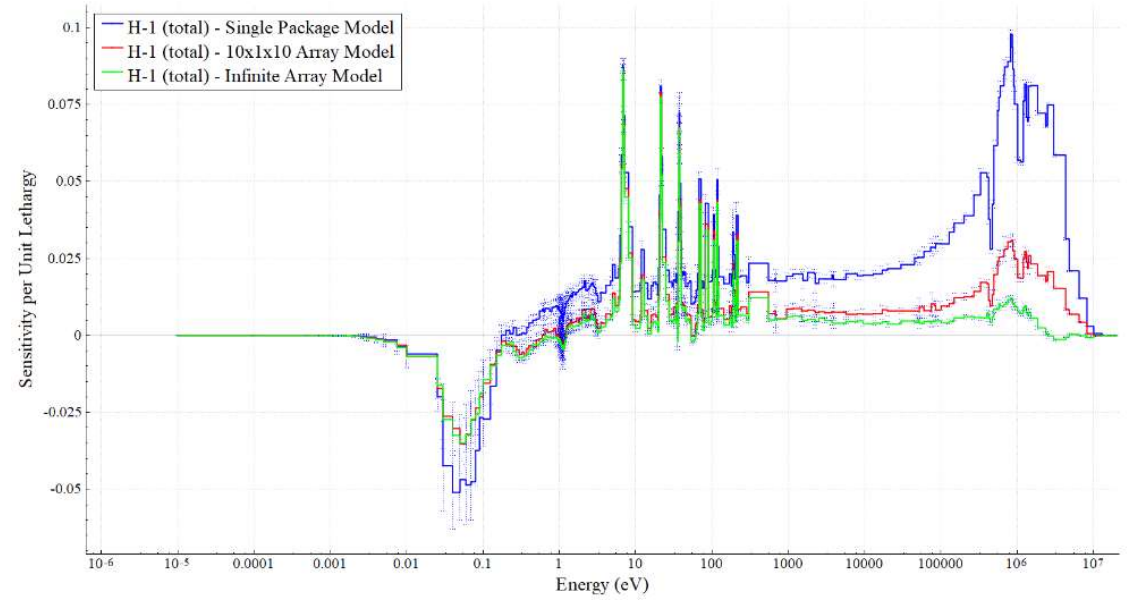

Figure C-2. Total ${ }^{1} \mathrm{H}$ sensitivity profiles for the three models.

The longer neutron lifetimes in the larger model also increase the magnitude of the ${ }^{238} \mathrm{U}$ sensitivity; more neutrons are absorbed by ${ }^{238} \mathrm{U}$ since there are no longer any neutrons leaking. The sensitivity profiles are shown in Figure C-3. The softening of the spectrum with the additional neutron scatter also reduces the fast fission contribution from ${ }^{238} \mathrm{U}$, as clearly shown in Figure C-3. 


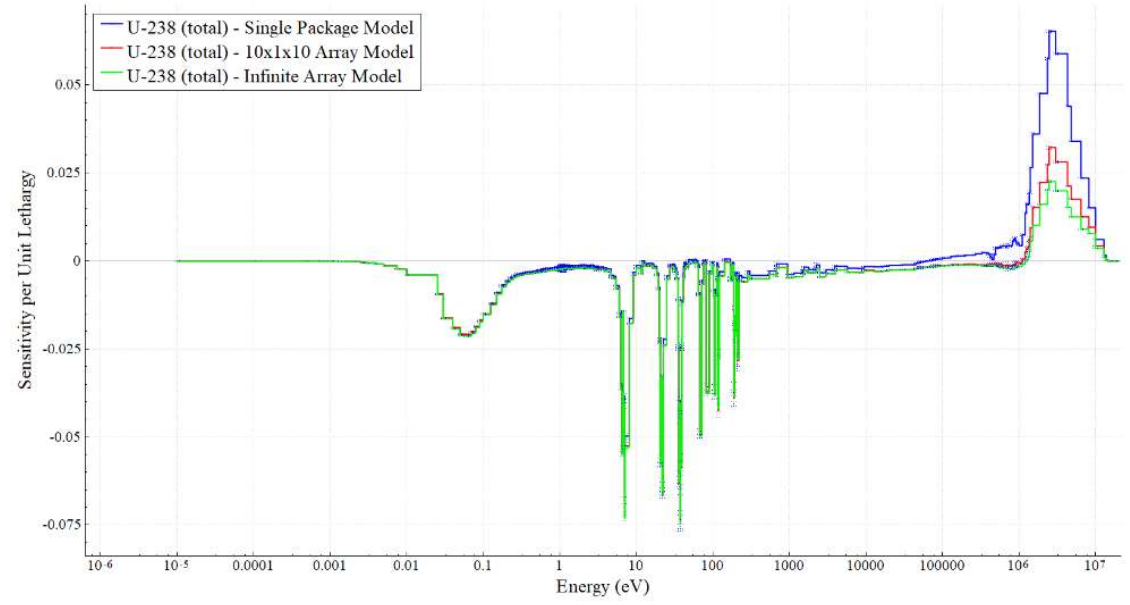

Figure C-3. Total ${ }^{238} \mathrm{U}$ sensitivity profiles for the three models.

The remaining question is how the differences in sensitivities will impact similarity assessments with available benchmark experiments. The results of the comparisons for these three models against the suite of 1,584 critical experiments are summarized in Table C-3 and shown in Figures C-4 through C-6. The figures illustrate that the pattern of $c_{k}$ values is similar, but the values drop approximately uniformly from the single package to the finite array to the infinite array models. The finite array model also shows less spread in $c_{k}$ values, especially for the LCT experiments, than either the single package or infinite array models.

Table C-3. Summary of similarity assessments for the 1,584 critical experiment suite.

\begin{tabular}{|c|c|c|c|}
\hline & $\begin{array}{c}\text { Single } \\
\text { package }\end{array}$ & $\mathbf{1 0 0}$ array & $\begin{array}{c}\text { Inf. } \\
\text { array }\end{array}$ \\
\hline $\begin{array}{c}\text { Median } \\
c_{k}\end{array}$ & 0.866 & 0.823 & 0.646 \\
\hline IEU & 0 & 0 & 0 \\
\hline LEU & 47 & 0 & 0 \\
\hline LCT & 518 & 167 & 12 \\
\hline LMT & 5 & 0 & 0 \\
\hline LST & 1 & 0 & 0 \\
\hline
\end{tabular}




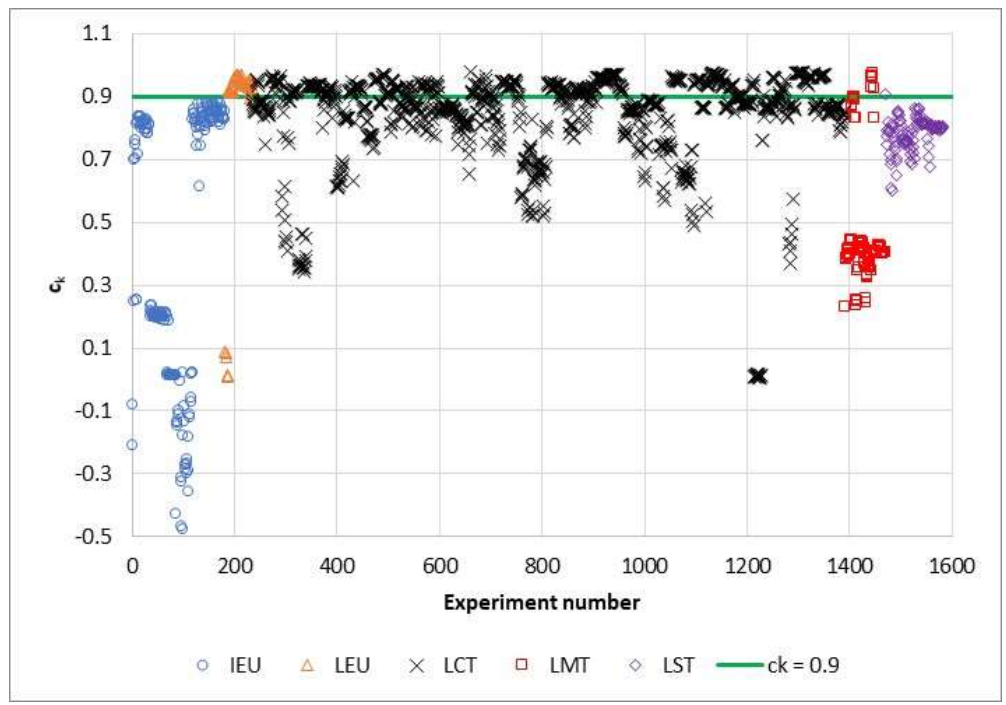

Figure C-4. $c_{k}$ values for single package model.

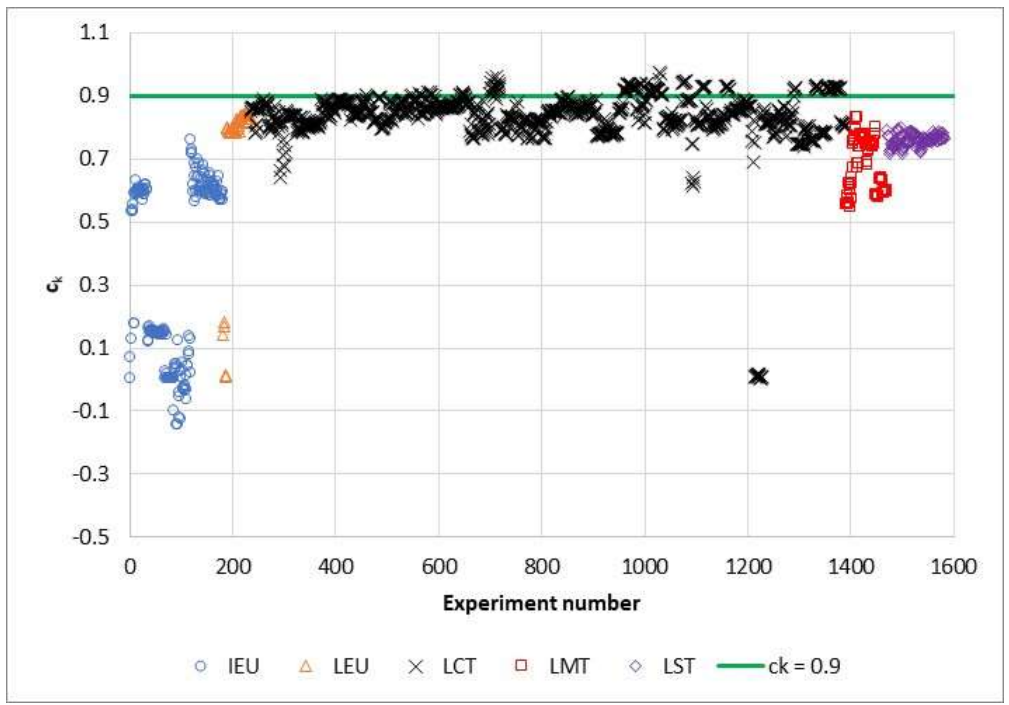

Figure C-5. $c_{k}$ values for $10 \times 1 \times 10$ package array. 


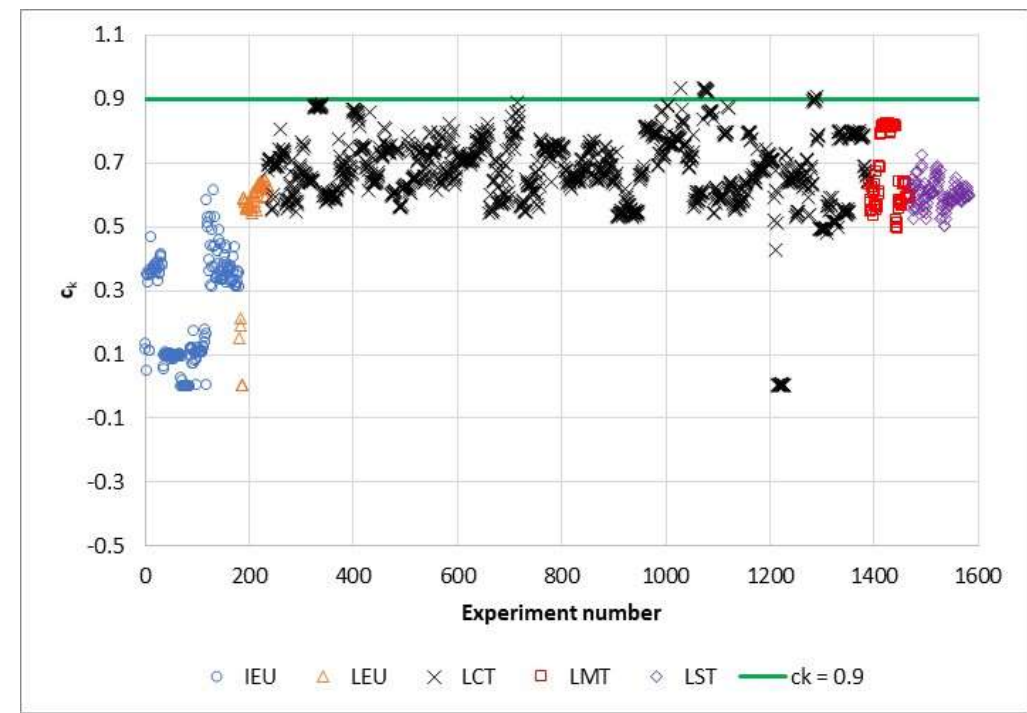

Figure C-6. $c_{k}$ values for infinite package array.

The integral parameter $c_{k}$ correlates the impact of nuclear data on $k_{\text {eff }}$ between systems, so an examination of the nuclear data-induced uncertainty and its top contributors for each system can help explain the differences observed in $c_{k}$ values. The total nuclear data-induced uncertainty for each application model and the top 10 contributors are shown in Table C-4. There are dramatic differences in the overall cross section uncertainty; the single package model uncertainly is more than $70 \%$ higher than the two array models. It is also clear that although the list of reactions contributing to the single package and infinite array models are quite different, the finite array model has a blend of the reactions that are prominent in the other two models. Therefore, both single unit models might predict applicable benchmarks for the HAC model, but the two models would do so for very different reasons. Neither model is likely to be a highly reliable indicator of benchmark applicability for the finite array model used in the safety basis analysis for this package.

Table C-4. Nuclear data-induced uncertainty for the three models.

\begin{tabular}{|c|c|c|c|c|c|c|}
\hline \multirow{3}{*}{$\begin{array}{c}\text { Total } \\
\text { Uncertainty }\end{array}$} & \multicolumn{2}{|c|}{ Single package } & \multirow{2}{*}{\multicolumn{2}{|c|}{$\frac{100 \text { array }}{0.536 \% \Delta k}$}} & \multicolumn{2}{|c|}{ Inf. array } \\
\hline & 0.92 & $\% \Delta k$ & & & 0.518 & $\% \Delta \mathrm{k}$ \\
\hline & Reaction & Unc $(\% \Delta k)$ & Reaction & Unc $(\% \Delta k)$ & Reaction & Unc $(\% \Delta k)$ \\
\hline Contributor \#1 & ${ }^{235} \mathrm{U} X$ & 0.587 & ${ }^{235} \mathrm{U} \bar{v}$ & 0.353 & ${ }^{235} \mathrm{U} \bar{v}$ & 0.355 \\
\hline Contributor \#2 & ${ }^{238} \mathrm{U}$ inel. & 0.486 & ${ }^{56} \mathrm{Fe}(\mathrm{n}, \gamma)$ & 0.182 & ${ }^{56} \mathrm{Fe}(\mathrm{n}, \gamma)$ & 0.207 \\
\hline Contributor \#3 & ${ }^{235} \mathrm{U} \bar{v}$ & 0.345 & ${ }^{238} \mathrm{U}(\mathrm{n}, \gamma)$ & 0.164 & ${ }^{238} \mathrm{U}(\mathrm{n}, \gamma)$ & 0.166 \\
\hline Contributor \#4 & ${ }^{16} \mathrm{O}$ elastic & 0.204 & ${ }^{235} \mathrm{U}(\mathrm{n}, \gamma)$ & 0.159 & ${ }^{235} \mathrm{U}(\mathrm{n}, \gamma)$ & 0.161 \\
\hline Contributor \#5 & ${ }^{238} \mathrm{U}$ inel./el. & -0.187 & ${ }^{235} \mathrm{U}$ fis $/(\mathrm{n}, \gamma)$ & 0.119 & ${ }^{235} \mathrm{U}$ fis/(n, $\left.\gamma\right)$ & 0.119 \\
\hline Contributor \#6 & ${ }^{238} \mathrm{U}(\mathrm{n}, \gamma)$ & 0.162 & ${ }^{235} \mathrm{U} X$ & 0.115 & ${ }^{235} \mathrm{U}$ fission & 0.113 \\
\hline Contributor \#7 & ${ }^{235} \mathrm{U}(\mathrm{n}, \gamma)$ & 0.158 & ${ }^{238} \mathrm{U}$ inel. & 0.114 & ${ }^{1} \mathrm{H}(\mathrm{n}, \gamma)$ & 0.079 \\
\hline Contributor \#8 & ${ }^{1} \mathrm{H}$ elastic & 0.148 & ${ }^{235} \mathrm{U}$ fission & 0.114 & ${ }^{238} \mathrm{U} \bar{\nu}$ & 0.051 \\
\hline Contributor \#9 & ${ }^{235} \mathrm{U}$ fis $/(\mathrm{n}, \gamma)$ & 0.115 & ${ }^{1} \mathrm{H}(\mathrm{n}, \gamma)$ & 0.082 & ${ }^{157} \mathrm{Gd}(\mathrm{n}, \gamma)$ & 0.044 \\
\hline Contributor $\# 10$ & ${ }^{235} \mathrm{U}$ fission & 0.108 & ${ }^{56} \mathrm{Fe}$ elastic & 0.058 & ${ }^{235} \mathrm{UX}$ & 0.041 \\
\hline
\end{tabular}




\section{APPENDIX D. COMPUTER INPUT AND OUTPUT FILES}

\section{Section 2}

Spreadsheets:

Modeling, tables, and plots: Traveller_TM_cases.xlsx

Direct perturbation vs. TSUNAMI sensitivity: DP Trav_asbly_5wt.xlsx, DP_Trav_asbly_8wt_IFBA.xlsx, DP_Trav_asbly_8wt_single_axial2, DP_Trav_RP_5wt.xlsx, DP_Trav_RP_5wt.xlsx

\section{Computer input and output:}

Excel spreadsheets and computer input and output for Traveller calculations are included in .zip file Traveller.zip.

\section{Section 3}

Spreadsheets (pellet and powder models):

Modeling, tables, and plots: CHT-OP-TU_model_data.xlsx

Direct perturbation vs. TSUNAMI sensitivity: DP_CHTOPTU_5wt_48_rev3.xlsx, DP_CHTOPTU_8wt_18_rev3_7-5in_rev3.xlsx, DP_CHTOPTU_18_6-9wt 7-5pel_rev3a.xlsx, DP_CHTOPTU_48_16-5wt_7-5pel_rev3.xlsx

\section{Computer input and output:}

Excel spreadsheets and computer input and output for CHT-OP-TU calculations are included in .zip file CHT-OP-TU.zip. Some 8 wt. $\% 3 \times 3 \times 2$ files are misnamed with 48 indicating 48 packages rather than the correct number of 18 packages.

\section{Section 5}

\section{Spreadsheets:}

Modeling, tables, and plots: TN-B1_data.xlsx

Direct perturbation vs. TSUNAMI sensitivity: DP_HAC_5wt_13Gd_10x10.xlsx, DP_HAC_67wt_13Gd_6x6.xlsx, DP_HAC_8wt_24Gd_10x10a.xlsx

\section{Computer input and output:}

Excel spreadsheets and computer input and output for TN-B1 calculations are included in .zip file TN-B1.zip.

\section{Section 6}

\section{Spreadsheets:}

Modeling, tables, and plots: UF6_sampler_data.xlsx, DN30_TSUNAMI_results.xlsx

Direct perturbation vs. TSUNAMI sensitivity: DP_DN30_5wt_inf.xlsx, DP_DN30_6-7wt_3x2.xlsx, DP_DN30_12-5wt_sgl.xlsx

\section{Computer input and output:}

Excel spreadsheets and computer input and output for TN-B1 calculations are included in .zip file DN30.zip.

\section{Appendices}

Spreadsheets: Combined_front_end_TSUNAMI_results_rev1, TN-B1_AppendixC.xlsx 